\title{
INVESTIGATION OF THE RECEPTORIAL AND MOLECULAR MEDIATORS OF SYSTEMIC INFLAMMATION IN DIFFERENT ANIMAL MODELS
}

Ph.D. Thesis

\section{Eszter Garaminé Pákai}

\author{
Doctoral School of Clinical Medicine \\ Faculty of Medicine, University of Szeged \\ Director: Lajos Kemény, M.D., Ph.D., D.Sc.
}

Translational Medicine Program

Director: Péter Hegyi, M.D., Ph.D., D.Sc.

Supervisor:

András Garami, M.D., Ph.D.

Department of Thermophysiology, Institute for Translational Medicine

Medical School, University of Pécs

Szeged

2020 


\section{Publications related to the subject of the thesis}

I. Pakai E, Tekus V, Zsiboras C, Rumbus Z, Olah E, Keringer P, Khidhir N, Matics R, Deres L, Ordog K, Szentes N, Pohoczky K, Kemeny A, Hegyi P, Pinter E, Garami A. The neurokinin-1 receptor contributes to the early phase of lipopolysaccharide-induced fever, IMMUNOLOGY 9 pp. 166, 15 p. (2018) IF: 4.716

II. Pakai E, Garami A, Nucci TB, Ivanov AI, Romanovsky AA. Hyperbilirubinemia exaggerates endotoxin-induced hypothermia, CELL CYCLE 14:8 pp. 1260-1267, 8 p. (2015) IF: 3.952

III. Banki E*, Pakai E*, Gaszner B, Zsiboras Cs, Czett A, Bhuddi PRP, Hashimoto H, Toth G, Tamas A, Reglodi D, Garami A. Characterization of the thermoregulatory response to pituitary adenylate cyclase-activating polypeptide in rodents, JOURNAL OF MOLECULAR NEUROSCIENCE 54:3 pp. 543-554, 12 p. (2014) IF: $\mathbf{2 . 3 4 3}$

IV. Wanner SP, Garami A, Pakai E, Oliveira DL, Gavva NR, Coimbra CC, Romanovsky AA. Aging reverses the role of the transient receptor potential vanilloid-1 channel in systemic inflammation from anti-inflammatory to proinflammatory, CELL CYCLE 11:2 pp. 343-349, 7 p. (2012) IF: $\mathbf{5 . 3 2 1}$

V. Garami A, Pakai E, Oliveira DL, Steiner AA, Wanner SP, Almeida MC, Lesnikov VA, Gavva NR, Romanovsky AA. Thermoregulatory phenotype of the trpv1 knockout mouse: thermoeffector dysbalance with hyperkinesis, JOURNAL OF NEUROSCIENCE 31:5 pp. 1721-1733, 13 p. (2011) IF: 7.115

*Authors contributed equally to this work.

\begin{tabular}{|ll|}
\hline Number of publications related to the subject of the thesis: & $\mathbf{5}$ \\
Number of publications not related to the subject of the thesis: & $\mathbf{9}$ \\
Number of book chapters: & $\mathbf{2}$ \\
Cumulative impact factor: & $\mathbf{5 9 . 8 6 5}$ \\
\hline
\end{tabular}




\section{Table of Contents}

List of abbreviations

$\begin{array}{ll}\text { 1. Introduction and literature background } & 6\end{array}$

1.1. Significance and appearance of systemic inflammation 6

1.2. Alterations of body temperature $\left(\mathrm{T}_{\mathrm{b}}\right)$ in systemic inflammation 7

1.3. Neurohumoral factors in the pathophysiology of systemic inflammation 9

1.4 Animal models to study systemic inflammation $\quad 12$

2. Aims of the study 13

3. Materials and methods $\quad 14$

$\begin{array}{ll}\text { 3.1. Experimental animals and their housing } & 14\end{array}$

$\begin{array}{ll}\text { 3.2. Surgical preparation } & 14\end{array}$

3.2.1. Intravenous, intraperitoneal catheterization and $\begin{array}{ll}\text { intracerebroventricular cannulation } & 15\end{array}$

3.2.2. Implantation of temperature-measuring device 16

3.2.3. CLP procedure 16

3.3. Experimental setups 16

3.3.1. Thermocouple setup 16

$\begin{array}{ll}\text { 3.3.2. Respirometry setup } & 17\end{array}$

$\begin{array}{ll}\text { 3.3.3. Telemetry setup } & 17\end{array}$

$\begin{array}{ll}\text { 3.4. Drugs and drug administration } & 17\end{array}$

$\begin{array}{ll}\text { 3.5. In vitro experiments } & 18\end{array}$

$\begin{array}{lr}\text { 3.6. Statistical analysis } & 19\end{array}$

$\begin{array}{ll}\text { 4. Results } & 20\end{array}$

4.1. The role of bilirubin in systemic inflammation 20

4.1.1. Lipopolysaccharide (LPS)-induced fever and hypothermia in Gunn rats $\quad 20$

4.1.2. Plasma bilirubin level in Gunn rats 20

4.1.3. Renal dysfunction and hepatic damage in Gunn

$\begin{array}{ll}\text { rats after LPS administration } & 22\end{array}$ 
4.2. The role of transient receptor potential vanilloid-1 (TRPV1)

channels in thermoregulation under physiological conditions and in systemic inflammation

4.2.1. Significance of TRPV1 channels in $T_{b}$ regulation and $\begin{array}{ll}\text { thermoeffector activities } & 24\end{array}$

4.2.2. The role of TRPV1 channels in aseptic or microbial sepsis 26

4.3. Characterization of the thermoregulatory effects of pituitary adenylate cyclase-activating polypeptide (PACAP)

4.3.1. Aspects of the thermoregulatory response to central and systemic PACAP38 administration in rats

4.3.2. Characteristics of the thermoregulatory response in the absence of PACAP 31

4.4. The role of the neurokinin-1 receptor in systemic inflammatory processes 33

4.4.1. Characteristics of the thermoregulatory response of Tacrl $^{+/+}$and Tacrl $^{-/-}$mice to LPS

4.4.2. LPS-induced changes in serum cytokines levels and cyclooxygenase-2 expression in $\mathrm{Tacr}^{+/+}$and $\mathrm{Tacr} \mathrm{I}^{-/-}$mice

5. Discussion

6. Summary and conclusions

Acknowledgements

References

Appendices I-V. 


\section{List of abbreviations}

\begin{tabular}{|c|c|}
\hline ALT & alanine aminotransferase \\
\hline AST & aspartate aminotransferase \\
\hline BAT & brown adipose tissue \\
\hline BUN & blood urea nitrogen \\
\hline CLP & cecal ligation and puncture \\
\hline CNS & central nervous system \\
\hline $\mathrm{COX}$ & cyclooxygenase \\
\hline GABA & gamma-aminobutyric acid \\
\hline GGT & gamma-glutamyl transferase \\
\hline HLI & heat loss index \\
\hline i.c.v. & intracerebroventricular(ly) \\
\hline i.p. & intraperitoneal(ly) \\
\hline i.v. & intravenous(ly) \\
\hline IL & interleukin \\
\hline $\mathrm{KO}$ & knockout \\
\hline LPS & lipopolysaccharide \\
\hline $\mathrm{MnPO}$ & median preoptic nucleus \\
\hline MPO & medial preoptic area \\
\hline NK1 & neurokinin-1 \\
\hline PACAP & pituitary adenylate cyclase-activating polypeptide \\
\hline $\mathrm{PE}$ & polyethylene \\
\hline PG & prostaglandin \\
\hline POA & preoptic area \\
\hline ROS & reactive oxygen species \\
\hline s.c. & subcutaneous(ly) \\
\hline SIRS & systemic inflammatory response syndrome \\
\hline SP & substance $\mathrm{P}$ \\
\hline $\mathrm{T}_{\mathrm{a}}$ & ambient temperature \\
\hline $\mathrm{T}_{\mathrm{ab}}$ & abdominal temperature \\
\hline $\mathrm{T}_{\mathrm{b}}$ & body temperature \\
\hline $\mathrm{T}_{\mathrm{c}}$ & colonic temperature \\
\hline TNF & tumor necrosis factor \\
\hline TNZ & thermoneutral zone \\
\hline TRPV1 & transient receptor potential vanilloid-1 \\
\hline $\mathrm{T}_{\mathrm{sk}}$ & tail skin temperature \\
\hline $\mathrm{VO}_{2}$ & oxygen consumption \\
\hline
\end{tabular}




\section{Introduction and literature background}

\subsection{Significance and appearance of systemic inflammation}

Systemic inflammation is a generalized pathological process, which can be clinically manifested in various forms, including sickness syndrome, systemic inflammatory response syndrome (SIRS), sepsis, severe sepsis, septic shock, and multiple organ dysfunction syndrome [1]. The inflammatory response is a series of complex pathophysiological events that can result from infections, as well as from non-infectious causes, such as trauma, burns, and pancreatitis $[2]$.

The exact definitions and clinical criteria of sepsis and related diseases have changed over the past three decades [3-5]. In 1991, a Consensus Conference was held with the goal of agreeing on definitions for the abovementioned terms. Such standardization of terminology was necessary to eliminate confusion in communication for clinicians and basic researchers concerning the phenomena related to systemic inflammation. For example, the term sepsis was generally used as a clinical response arising from infection. However, similar, or even identical, clinical responses can also arise in the absence of infection. As result of the consensus in 1991, the phrase SIRS was proposed to describe this inflammatory process, independent of its cause. In cases, when SIRS results from a confirmed (or suspected) infectious process, it is termed sepsis, since then it represents the systemic inflammatory response to the infection. Definition of SIRS includes two or more of the following conditions: (1) body temperature $\left(\mathrm{T}_{\mathrm{b}}\right)>38^{\circ} \mathrm{C}$ or $<36^{\circ} \mathrm{C}$; (2) heart rate $>90$ beats per minute; (3) respiratory rate $>20$ breaths per minute or $\mathrm{PaCO}_{2}<32 \mathrm{~mm} \mathrm{Hg}$; and (4) white blood cell count $>12000 / \mathrm{mm}^{3},<4000 / \mathrm{mm}^{3}$, or $>10 \%$ immature bands [3]. In 2001, the definitions and criteria were revised, while the list of signs and symptoms of sepsis was expanded to better reflect the clinical response to infection, no new definitions for sepsis were introduced [4]. In line with the actuality of the topic, in 2015, some concepts have been changed. For instance, sepsis was defined as life-threatening organ dysfunction caused by an improperly regulated host response to infection. Septic shock was determined as a subset of sepsis in which particularly profound circulatory, cellular, and metabolic abnormalities are associated with a greater risk of mortality than with sepsis alone. Also, SIRS and severe sepsis were eliminated from the terms related to septic diseases [5]. 
According to a recent study, sepsis constitutes a global burden for health care, with an estimated 48.9 million incident cases and 11 million sepsis-related deaths worldwide in a year [6], moreover, it has an incidence rate of $\sim 30 \%$ in patients admitted to the intensive care unit [7]. It is important to mention, that sepsis can lead to death not only acutely, but it also increases the risk of death for five to eight years after the septic event $[8,9]$. In the United States, the incidence of hospitalizations with sepsis increased by 50\% between 2003 and 2009 [10], with estimated annual costs exceeding $\$ 17$ billion nationally due in part to the extended hospitalization of septic patients [11]. However, a more recent study describes, that the incidence of sepsis and sepsis-related mortality has remained stable in the United States between 2009-2014 [12].

\subsection{Alterations of $T_{b}$ in systemic inflammation}

As a systemic inflammation response, sepsis is often accompanied by changes of deep $\mathrm{T}_{\mathrm{b}}$, which can be manifested as either fever or hypothermia in experimental animals, as well as in human patients [1].

The importance of alterations in $\mathrm{T}_{\mathrm{b}}$ in inflammation was recognized a long time ago. Elevation of $\mathrm{T}_{\mathrm{b}}$ is also reflected in the word 'inflammation' itself, as it originates from the Latin inflammare (to set on fire). The presence of fever was already noted in the time of Hippocrates in disease states [13]. Heat (calor) is also one of the four cardinal signs of inflammation, along with redness, swelling, and pain, which were originally described by Celsus in the 1st century A.D. [14]. As an answer of the host's defense reactions to danger, the inflammatory response is usually accompanied by changes in vital functions in fever, including increased activity of white blood cells, heart rate, and respiratory rate. Fever helps inhibiting growth of many microorganisms, at the same time achiness, fatigue and sleepiness conserve energy that is needed to fight the infection and elevate $\mathrm{T}_{\mathrm{b}}$; moreover, loss of appetite minimizes blood glucose, which is a preferred fuel for many microorganisms [15]. However, in severe forms of sepsis the maintenance of fever is challenging for the host, therefore, the conservation of energy resources by reduced metabolism and hypothermia is the preferred response.

Based mainly on experimental data from animal studies it was proposed that fever and hypothermia can both develop as two definite adaptive mechanisms in sickness syndrome. 
Fever typically occurs at the onset of an infection, representing an active fight against the pathogen, while hypothermia is usually associated with progressed stage or severity of the disease and it aims to secure the vital systems of the host $[16,17]$. On the one hand, the two adaptive strategies can develop consecutively (e.g., early phase fever followed by late phase hypothermia) as the severity of the disease progresses; on the other hand, hypothermia can be also one of the earliest developing events in animal models of endotoxin shock [18]. Moreover, septic patients admitted to intensive care unit develop hypothermia more frequently in the early than in the late stages of their stay [19]. Therapeutic (i.e., induced) hypothermia has been also shown to improve the outcome of sepsis both in human and animal studies [20,21], however a positive association was found between mortality rate and spontaneously occurring hypothermia in several studies reporting that the mortality rate of hypothermic patients with sepsis is twice as high as that of febrile septic patients [22-24]. A recent meta-analysis on this topic also supported these findings, showing that fever predicts lower, while hypothermia higher mortality rates compared with normal $\mathrm{T}_{\mathrm{b}}$, based on data from over 10,000 septic patients [25]. It has to be noted that the prognostic value of hypothermia in sepsis does not automatically mean a negative effect of $T_{b}$ itself. Instead, the association between hypothermia and mortality can simply reflect the higher prevalence of hypothermia in severe cases of sepsis [26].

In accordance with the frequent thermoregulatory disorders that accompany systemic inflammation, many clinical scoring systems include the change of $\mathrm{T}_{\mathrm{b}}$. Among them, SIRS criteria are used for diagnosis [3, 4], while other scores are calculated to assess the prognosis of the critically ill patients, such as the Simplified Acute Physiology Score [27], the Acute Physiology and Chronic Health Evaluation [28], and the Predisposition, Insult, Response, Organ Dysfunction system [29]. A common disadvantage of these scoring systems is that reduced and elevated $T_{b S}$ are considered to be equal levels of concern [30], which contradicts the current view in the field that fever is associated with lower, and hypothermia with higher severity of a disease $[16,17]$.

Despite the different pathological background of fever and hypothermia in systemic inflammation, both the increase and the decrease of $\mathrm{T}_{\mathrm{b}}$ are evaluated as significant signs in the clinical practice. Thus, because of its frequent occurrence and high mortality rates, as of today, systemic inflammation represents a highly important field in basic research as well as in clinical practice. 


\subsection{Neurohumoral factors in the pathophysiology of systemic inflammation}

When the inflammation is triggered by an infectious insult, the inflammatory cascade is often initiated by an endotoxin, and then several signals activate the immune response. The most important mediators of systemic inflammation have been reviewed in details recently [1, $31]$.

As a summary, many immune system cells hold receptors for common macromolecules present on invading organisms. For instance, CD14, a human protein expressed mainly by macrophages as part of the immune system, detects and binds lipopolysaccharide [(LPS), the major component of the outer membrane of Gram-negative bacteria] and activates Toll-like receptor 4, which triggers an intracellular multi-step process activating immune responses [32]. In this immune response cytokines are released, including tumor necrosis factor (TNF)- $\alpha$ [33], interleukin (IL)-1 $\beta$, IL-6, and other mediators, cumulatively referred to as the "inflammatory soup" [34-36] and either attract other immune cells to the site of the inflammation, or act on other peripheral tissues to release further chemical mediators of inflammation.

Among these mediators are the prostaglandins (PGs), which have a crucial role in mediating many aspects of systemic inflammation. PGs are derivatives of arachidonic acid, which is converted to $\mathrm{PGH}_{2}$ by cyclooxygenase (COX) enzyme, which has two isoforms, $\mathrm{COX}$ 1 and $\mathrm{COX}-2$. Various additional enzymes convert $\mathrm{PGH}_{2}$ to end-products: other $\mathrm{PGs}\left(\mathrm{PGE}_{2}\right.$ and $\mathrm{PGD}_{2}$ ), prostacyclins or leukotrienes. $\mathrm{PGE}_{2}$ acts on four different EP receptors, which are expressed in different parts of the central nervous system (CNS) [15, 37].

In studies conducted on rodents, it has been shown that in response to low-dose of LPS fever develops mainly through the activation of COX-2 [38] and that $\mathrm{PGE}_{2}$ acts through EP3 receptors as the major mechanism in the development of the febrile response [39]. In contrast with fever, when a high dose of LPS is injected to rats hypothermia develops, which is triggered mainly by the activation of the COX-1 enzyme [38]. Receptorial and intracellular signaling cascades activated by the endogenous mediators are complex and involve lipid, peptide, gaseous, and other - as of today unidentified - messengers [40].

As common and prominent biochemical part of the inflammatory response, reactive oxygen species (ROS) are also produced. While production of ROS by different cells and tissues challenged with bacterial pyrogens is well documented [41], a little is known about 
causal roles of ROS in regulating $\mathrm{T}_{\mathrm{b}}$ responses during inflammation and infection, mainly due to the lack of effective tools for such investigations. Bilirubin, as a lipophilic molecule is generally considered as potentially toxic waste that needs to be excreted. However, some studies highlighted bilirubin as a powerful antioxidant that efficiently scavengers lipoperoxid radicals generated in either artificial or cellular membranes, and emphasizes its cytoprotective role $[42,43]$. Hence, bilirubin might be used as a selective tool to inactivate water insoluble, lipid-generated ROS.

In addition to ROS, studies have brought attention to the role that the transient receptor potential vanilloid-1 (TRPV1) channel expressed on sensory neural fibers may play a role in different inflammatory processes (Fig. 1) [44-46].

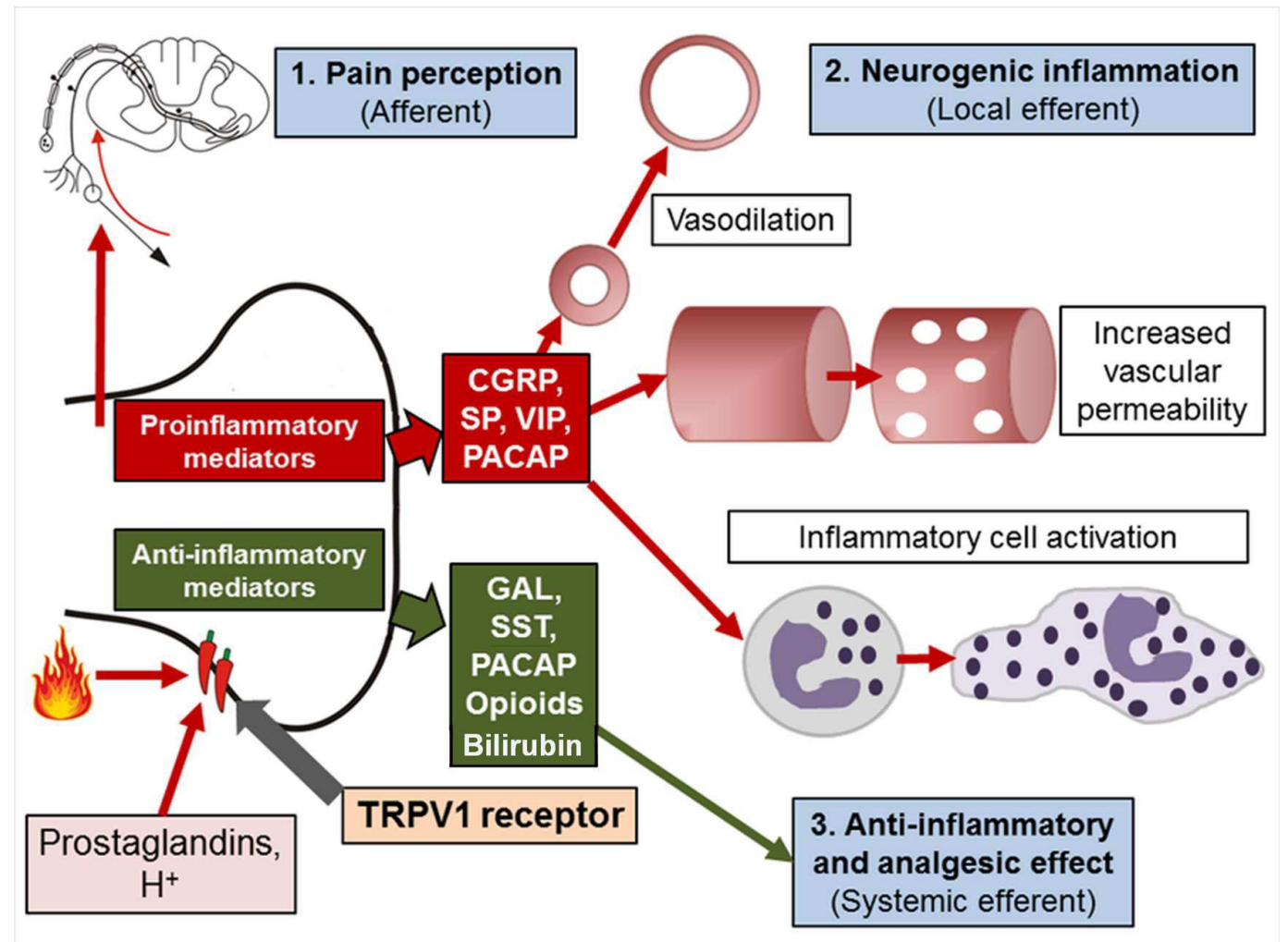

Figure 1. The three distinct effects of the activation of capsaicin-sensitive sensory nerve endings. CGRP, calcitonin generegulating polypeptide; GAL, galanin; PACAP, pituitary adenylate cyclase-activating polypeptide; SP, substance P; SST, somatostatin; TRPV1, transient receptor potential vanilloid-1; VIP, vasoactive intestinal polypeptide [44]. 
TRPV1 is activated by diverse stimuli, including several ingredients of the inflammatory soup [47]. Studies using either knockout (KO) (Trpv1/-) mice, a pharmacological blockade with capsazepine (TRPV1 antagonist) or desensitization with resiniferatoxin (TRPV1 agonist) have shown that TRPV1 plays an anti-inflammatory role in LPS-induced SIRS by, among other mechanisms, limiting the production of TNF- $\alpha$, possibly via sensory nerves [48, 49]. However, most studies were conducted in young rodents. Because SIRS is considered a disease of the aged due to its markedly higher incidence in the older population, it is also important to clarify whether TRPV1 channels play a similarly prominent anti-inflammatory role in young and older populations.

Performing experiments to elucidate the role of TRPV1 in systemic inflammation also implies the use of genetically modified $\left(\operatorname{Tr} p v 1^{-/-}\right)$mice, but according to the literature, studies in genetically modified animals have failed to reveal a clear thermoregulatory phenotype in those mice [47]. Therefore, it is essential to first clarify the thermoregulatory phenotype of the $\operatorname{Trp} v 1^{-/-}$mice under normal conditions and so we could determine whether these mice are suitable for the study of the role of TRPV1 in systemic inflammation.

The activation of TRPV1 channels on neural endings (i.e., on capsaicin-sensitive afferent nerve fibers) also results in the release of an excess of inflammatory substances such as pituitary adenylate cyclase-activating polypeptide (PACAP)38, calcitonin gene-related peptide, and substance P (SP) (Fig. 1).

PACAP38 deserves special attention, because the complex role of this, relatively newly discovered, peptide has been well established in various inflammatory processes [50-52]. Moreover, the peptide and its receptors are broadly expressed in main thermoregulatory areas of the brain, including the preoptic area (POA) of the hypothalamus [53-56], which is an important site in the development of the fever response. In physiological studies, injection of PACAP38 into the CNS caused an increase of $\mathrm{T}_{\mathrm{b}}$ in rodents, which was brought about by elevation of non-shivering thermogenesis and increased locomotor activity $[57,58]$, but it has remained unclear whether PACAP38 can lead to simultaneous activation of autonomic thermoeffectors, which would be in accordance with a fever-like response. To fill this gap and to study whether the release of PACAP38 from TRPV1-expressing neurons can be regarded as a mediator of systemic inflammation, as a part of our investigation we aimed to characterize the thermoregulatory effects of PACAP38 on deep $\mathrm{T}_{b}$. 
Since the neurokinin-1 (NK1) receptor, formerly known as SP receptor, also plays an important role in mediation of local and systemic inflammatory processes, SP signaling has been presumed as mediator of fever. When rodents were treated with peptide SP (antagonist) analogs, the fever response to LPS was blocked [59, 60], similar attenuation of the LPS-induced fever was observed in rats after administration of the NK1 receptor blockers $[61,62]$. These studies strongly support that SP signaling contributes to the development of LPS-induced fever, but it has remained largely unknown which mediators of the febrile process are influenced by SP or its receptors. Alternative approaches, such as the use of KO mice, may help to better clarify which step(s) of the classical molecular mechanisms of fever are influenced by the NK1 receptor.

\subsection{Animal models to study systemic inflammation}

SIRS can be either triggered by non-infectious insults, or it can be associated with an infection, when it is called sepsis. To study the etiology of sepsis in the laboratory, different animal models have developed.

Cecal ligation and puncture (CLP), a murine model of bacterial peritonitis has been used extensively to investigate the clinical settings of polymicrobial sepsis and septic shock [63]. Systemic administration of high or low dose of LPS in mice and rats is often used to induce SIRS aseptically. In the CLP and high-dose LPS models, shock and death can occur, largely as the result of the "cytokine storm," a definite production of proinflammatory cytokines [35]. 


\section{Aims of the study}

The ultimate aim of the study was to discover new mechanisms, which may play a part in the mediation of systemic inflammation. Since systemic inflammation is often accompanied by alterations in deep $T_{b}$, in most experiments we used a thermophysiological approach to explore the contribution of the different investigated mediators to inflammation.

To better understand the pathophysiological background of the inflammatory processes, in our study we wanted to investigate the role(s) of endogenous substances (which are physiologically produced in the body) and receptorial mechanisms related to the development of systemic inflammation.

Therefore, because of their established roles in inflammatory processes, in different animal models:

- we focused a part of our study on the role of bilirubin in systemic inflammation;

- we attempted to characterize the role of the TRPV1 channel in thermoregulation under physiological conditions and in systemic inflammation;

- we wanted to elucidate the importance of the TRPV1 channel in aseptic and microbial sepsis;

- we studied the influence of PACAP on thermoregulatory processes;

- $\quad$ and we analyzed the role of the NK1 receptor in inflammatory mechanisms.

Here we provide a summary of the most important aims and findings, while the detailed description of the original studies can be found in the papers that served as the basis of the present work [64-68] (for full texts, see Appendices I-V.). 


\section{Materials and methods}

\subsection{Experimental animals and their housing}

Over the past decades, the rat has become the primary animal model to study thermoregulatory manifestations of systemic inflammation, but with rapid development of genetic manipulations and new species-specific tools (recombinant antibodies and other proteins), the mouse is also becoming a common species to investigate the mechanisms of fever and hypothermia [16].

In our experiments we used adult male Wistar rats, as well as normobilirubinemic (heterozygous, asymptomatic; J/+) and hyperbilirubinemic (homozygous, jaundiced; J/J) Gunn rats. Other studies were conducted in mice of both sexes with the Trpvl gene either present $\left(\operatorname{Trp} v 1^{+/+}\right)$or missing $\left(\operatorname{Trp} v 1^{-/-}\right)$; or with the Tacrl gene (i.e., the gene encoding the NK1 receptor) homozygously either present $\left(\right.$ Tacr $\left.^{+/+}\right)$or absent $\left(\right.$Tacr $\left.^{-/-}\right)$; or with the Pacap gene homozygously either present $\left(\mathrm{Pacap}^{+/+}\right)$or absent $\left(\right.$Pacap $\left.^{-/}\right)$.

The animals were housed under standard conditions: kept in temperature-controlled rooms on a $12 \mathrm{~h}$ light/dark cycle, where standard rodent chow and tap water were available $a d$ libitum. The animals were housed in groups until they were subjected to surgery, after which they were caged singly. They were extensively handled and habituated to staying in wire-mesh conical confiners, which were later used in the experiments. All procedures were conducted under protocols approved by the Institutional Animal Care and Use Committees.

\subsection{Surgical preparation}

Surgeries were performed under ketamine-xylazine $[55.6$ and $5.5 \mathrm{mg} / \mathrm{kg}$ intraperitoneally (i.p.), respectively] anesthesia on rats, and under ketamine-xylazine (81.7 and $9.3 \mathrm{mg} / \mathrm{kg}$ i.p., respectively) anesthesia on mice; in some centers also in combination with acepromazine (1.1 and $1.2 \mathrm{mg} / \mathrm{kg}$ for rats and mice, respectively); for both species, antibiotic protection [gentamycin, $6 \mathrm{mg} / \mathrm{kg}$, intramuscularly or enrofloxacin, $1.1 \mathrm{mg} / \mathrm{kg}$, subcutaneously (s.c.)] was provided, except for animals subjected to CLP. For pain management, ketoprofen or 
carprofen $(5 \mathrm{mg} / \mathrm{kg}$, s.c.) was administered at the end of surgery and on the next day. The experiments were performed 4-7 days after the surgeries.

3.2.1. Intravenous (i.v.), i.p. catheterization and intracerebroventricular (i.c.v.) cannulation

In order to administer substances to rats and mice in a non-stressful manner, the animals were preimplanted with an i.v. or i.p. catheter or with an i.c.v. cannula.

For implanting an i.v. catheter, a small longitudinal incision was made on the ventral surface of the neck, left to the trachea. The left jugular vein was exposed, freed from its surrounding connective tissue, and ligated. A silicone catheter (ID $0.5 \mathrm{~mm}$, OD $0.9 \mathrm{~mm}$ ) filled with heparinized saline $(50 \mathrm{U} / \mathrm{ml})$ was passed into the superior vena cava through the jugular vein and secured in place with ligatures. The free end of the catheter was knotted, tunneled under the skin to the nape, and exteriorized. The wound on the ventral surface of the neck was sutured. The catheters were flushed with heparinized saline every other day.

For the i.p. administration of drugs, a polyethylene (PE) catheter filled with pyrogenfree saline was inserted into the peritoneal cavity, and the internal end of the catheter was attached with a suture to the abdominal wall. The free end of the catheter was knotted, tunneled under the skin to the nape, and exteriorized. The catheter was flushed with saline every other day.

For i.c.v. drug administration, a 22 ga steel guide cannula (Plastics One) was implanted into the right lateral brain ventricle. The head of the animal was fixed in a stereotaxic apparatus; the scalp was incised over the sagittal suture; the periosteum was excised; the bone surface was cleaned; and two supporting microscrews were driven into the skull. A hole was drilled 1.0/0.5 $\mathrm{mm}$ (rat/mouse, respectively) posterior to bregma and 1.5/1.0 $\mathrm{mm}$ (rat/mouse, respectively) right of the sagittal suture. The guide cannula was inserted into the lateral ventricle $3.8 / 2.0 \mathrm{~mm}$ (rat/mouse, respectively) below the skull surface. The cannula was fixed to the supporting microscrews with acrylic cement. After the experiments were concluded, a correct placement of each i.c.v. cannula was confirmed by anesthetizing the animal, infusing $5 \mu \mathrm{l}$ of an aqueous solution of methylene blue $(6 \mathrm{mg} / \mathrm{ml})$ through the cannula, removing the brain, and then examining coronal sections macroscopically for the presence of the dye in the ventricular 
system. Animals without the dye in the lateral cerebral ventricle were excluded from the final statistical analysis.

\subsubsection{Implantation of temperature-measuring device}

To record abdominal temperature $\left(\mathrm{T}_{\mathrm{ab}}\right.$, a measure of deep $\mathrm{T}_{\mathrm{b}}$ ) and gross locomotor activity in freely-moving mice, a miniature telemetry transmitter (G2 E-Mitter series; Mini Mitter) was implanted. The device was inserted into the peritoneal cavity via midline laparotomy and fixed to the lateral abdominal wall with a suture. The surgical wound was sutured in layers. Mice were then placed on top of the receivers (see section 3.3.3., for details) in their home cages.

\subsubsection{CLP procedure}

For CLP, the cecum was pulled out of the abdominal cavity, filled with the intestinal content (by gently squeezing the content from the ascending colon) and ligated with silk just distal to the ileocecal junction. The cecal wall was punctured through at the antimesenteric side with a needle. The survival rate and $\mathrm{T}_{\mathrm{b}}$ were monitored for $108 \mathrm{~h}$, at the end of experiments, any survivors were euthanized.

\subsection{Experimental setups}

We used three different experimental setups to measure the various thermophysiological parameters of rats and mice under physiological conditions and in forms of systemic inflammation. All experiments, were conducted under thermoneutral conditions, unless specified otherwise.

\subsubsection{Thermocouple setup}

The animals were placed in a confiner and equipped with two copper-constantan thermocouple (Omega Engineering) to measure colonic temperature $\left(\mathrm{T}_{\mathrm{c}}\right)$ and tail skin temperature $\left(\mathrm{T}_{\mathrm{sk}}\right)$. The colonic thermocouple was inserted 10 or $3 \mathrm{~cm}$ deep beyond the anal sphincter in rats and mice, respectively, and fixed to the tail with a loop of adhesive tape. The skin thermocouple was positioned on the lateral surface of the tail and insulated from the environment with tape. To record $\mathrm{T}_{\mathrm{b}}$ and $\mathrm{T}_{\mathrm{sk}}$ data, the thermocouple was plugged into a data logger (Cole-Parmer) connected to a computer. The animal in its confiner was then placed in 
an environmental chamber (Forma Scientific) set to a neutral ambient temperature $\left(T_{a}\right)$ specific for the setup. The implanted catheter or cannula was extended with a length of PE-50 tubing filled with saline, which was passed through a wall port and connected to a syringe filled with the drug of interest or saline.

\subsubsection{Respirometry setup}

Each animal in its confiner was transferred to a Plexiglas chamber of the four-chamber open-circuit calorimeter integrated system (Oxymax Equal Flow, Columbus Instruments, Columbus, $\mathrm{OH}, \mathrm{USA}$ ). The chamber was sealed, submerged into a temperature-controlled water bath, and continuously ventilated with room air. The fractional concentration of oxygen was measured in the air entering and exiting the chamber, and the rate of oxygen consumption $\left(\mathrm{VO}_{2}\right)$ was calculated according to the manufacturer's instructions using the Oxymax Windows software. In another setup, animals in their confiners were placed inside a sealed cylindrical Plexiglas chamber (Sable Systems). The Plexiglas chambers were kept in a climatic chamber and continuously ventilated. The rate of $\mathrm{VO}_{2}$ was calculated by comparing the oxygen fraction in the air exiting the chamber occupied by an animal to the oxygen fraction in the air exiting an empty chamber.

\subsubsection{Telemetry setup}

In this setup, mice were studied inside their home cages. Telemetry receivers (model ER-4000; Mini Mitter) were positioned in a temperature-controlled room or in a climatic chamber, and the home cages of mice preimplanted with a telemetry transmitter were placed on top of the receivers in order to measure $\mathrm{T}_{\mathrm{ab}}$ and locomotor activity in freely moving animals.

\subsection{Drugs and drug administration}

LPS from Escherichia coli 0111:B4 was purchased from Sigma-Aldrich. A stock suspension of LPS $(5 \mathrm{mg} / \mathrm{ml})$ in pyrogen-free saline was stored at $-20^{\circ} \mathrm{C}$. To induce SIRS in experimental animals, LPS was injected in a bolus $(10$ or $1,000 \mu \mathrm{g} / \mathrm{kg}$ i.v. for rats; 0.12 or 40 $\mathrm{mg} / \mathrm{kg}$ i.p. for mice) through the extension of the i.v. or i.p. catheter. Variables of interest were monitored up to 7 hours after the injection. Controls received saline. 
AMG 517, which is a highly potent and selective TRPV1 antagonist, was used to block TRPV1 receptors pharmacologically. Aliquots of an ethanolic solution of AMG $517(3 \mathrm{mg} / \mathrm{ml})$ were stored at $-80^{\circ} \mathrm{C}$. AMG $517(210 \mu \mathrm{g} / \mathrm{kg}$ s.c. $)$ or its vehicle was administered as a bolus, $1 \mathrm{~h}$ before the administration of the pyrogen agent or saline.

PACAP38 was synthesized at the University of Szeged as described in details elsewhere [69]. Lyophilized aliquots of PACAP38 were stored at $4^{\circ} \mathrm{C}$. On the day of the experiment, a working solution was prepared and PACAP38 was delivered at final doses of $100 \mu \mathrm{g} / \mathrm{kg}$ i.v. and at 10 and $100 \mu \mathrm{g} / \mathrm{kg}$ i.c.v. for rats. Control animals were infused with saline.

SP was purchased from Tocris Bioscience, Bristol, UK. Aliquots of an ethanolic stock solution of SP were stored at $-80^{\circ} \mathrm{C}$. On the day of the experiment, the stock solution was diluted with saline and infused into the lateral ventricle, at a total dose of $100 \mu \mathrm{g} / \mathrm{kg}$. Controls were infused with the vehicle (10\% ethanol in saline).

\subsection{In vitro experiments}

Levels of total bilirubin and markers of renal dysfunction and hepatocyte damage were determined in blood samples collected by cardiac puncture from rats $24 \mathrm{~h}$ after LPS injection. The blood was immediately transferred to EDTA-containing Vacutainer tubes. The plasma was separated by centrifugation, aliquoted, and stored at $-80^{\circ} \mathrm{C}$ until biological assays were performed.

Immunohystochemistry for the labeling of c-fos-expressing neurons in the Pacap $^{-/-}$and Pacap $^{+/+}$mice was performed as described in details elsewhere [70]. In brief, the cell counts positive for c-Fos were determined in five serial sections, each interspaced by $60 \mu \mathrm{m}$ in the median preoptic nucleus (MnPO) and medial preoptic area (MPO) according to Paxinos and Franklin atlas [71]. Cell counting was carried out on non-edited digital images and quantitation was performed in a double-blind setup.

In order to determine LPS-induced changes in COX-2, 40 minutes after LPS infusion each mouse was perfused, lung, liver and brain tissue samples were collected and snap frozen in liquid nitrogen for RT-qPCR and Western blot. All tissue samples were stored at $-80^{\circ} \mathrm{C}$. 


\subsection{Statistical analysis}

Blood levels of bilirubin and biochemical markers of renal and hepatic dysfunction, and numbers of the c-Fos-positive cells were compared with Student's t test.

Thermophysiological parameters and COX-2 expression were compared by two- or three-way ANOVA followed by Fisher's LSD post hoc tests or by Student's t test, as appropriate.

Survival data were analyzed by the $\chi 2$ test for individual time points and by the logrank test for the entire observation period. The Cox proportional hazard survival regression model was used to determine hazard ratios of death.

For statistical analysis, Sigmaplot 11.0 or Statistica AXA 8.0 software was used. The effects were considered significant when $\mathrm{P}<0.05$. All data are reported as mean \pm standard error. 


\section{Results}

\subsection{The role of bilirubin in systemic inflammation}

\subsubsection{LPS-induced fever and hypothermia in Gunn rats}

At $30^{\circ} \mathrm{C}$, rats respond to the low dose of LPS with a polyphasic fever, and to the high dose with hypothermia followed by fever [16]. In our study with Gunn rats [66], the i.v. injection of saline did not affect the $\mathrm{T}_{\mathrm{c}}$ in either genotype of rats (Fig. 2A). To the low dose (10 $\mu \mathrm{g} / \mathrm{kg}$ ) of LPS, both $\mathrm{J} / \mathrm{J}$ and $\mathrm{J} /+$ rats responded with a triphasic fever with peaks at $\sim 50,130$, and 280 min post-injection (Fig. 2B), however there were no significant differences in the $\mathrm{T}_{\mathrm{b}}$ dynamics between the genotypes. To the high dose $(1,000 \mu \mathrm{g} / \mathrm{kg})$ of LPS, J/+ rats responded with early hypothermia (nadir of $-0.5^{\circ} \mathrm{C}$ at $\sim 90 \mathrm{~min}$; $\mathrm{P}=0.034 \mathrm{vs}$. saline) followed by fever (peak of $0.9^{\circ} \mathrm{C}$ at $\sim 360 \mathrm{~min} ; \mathrm{P}<0.001$ vs. saline) (Fig. $2 \mathrm{C}$ ). A different $\mathrm{T}_{\mathrm{b}}$ response of $\mathrm{J} / \mathrm{J}$ rats was observed to the high dose of LPS, the early hypothermic response (nadir of $-1.2^{\circ} \mathrm{C}$ at $\sim 90 \mathrm{~min}$, $\mathrm{P}<0.001$ vs. saline) was remarkably exaggerated ( $\mathrm{P} \leq 0.004$ for $40-180 \mathrm{~min}$ vs. $\mathrm{J} /+$ rats $)$, and the subsequent fever response (peak of $0.3^{\circ} \mathrm{C}, \mathrm{P}=0.039$ vs. saline) was significantly attenuated $(\mathrm{P}<0.05$ for $240-420$ min vs. $\mathrm{J} /+$ rats $)$.

\subsubsection{Plasma bilirubin level in Gunn rats}

As LPS administration may cause liver failure with hyperbilirubinemia, we examined blood bilirubin levels in $\mathrm{J} / \mathrm{J}$ and $\mathrm{J} /+$ rats under basal conditions and in LPS-induced systemic inflammation [66]. As expected, the total plasma bilirubin level in saline-treated $\mathrm{J} / \mathrm{J}$ rats was

significantly higher (by 2 orders of magnitude) than that in $\mathrm{J} /+$ rats, moreover the low dose of LPS did not affect the total bilirubin level in either genotype. However, in response to the injection of the high dose of LPS, the total bilirubin level elevated in both $\mathrm{J} /+$ and $\mathrm{J} / \mathrm{J}$ rats $(\mathrm{P}<0.001$ vs. saline, for both genotypes), while in $\mathrm{J} / \mathrm{J}$ rats it has still remained higher than in $\mathrm{J} /+$ controls (figure not shown). 

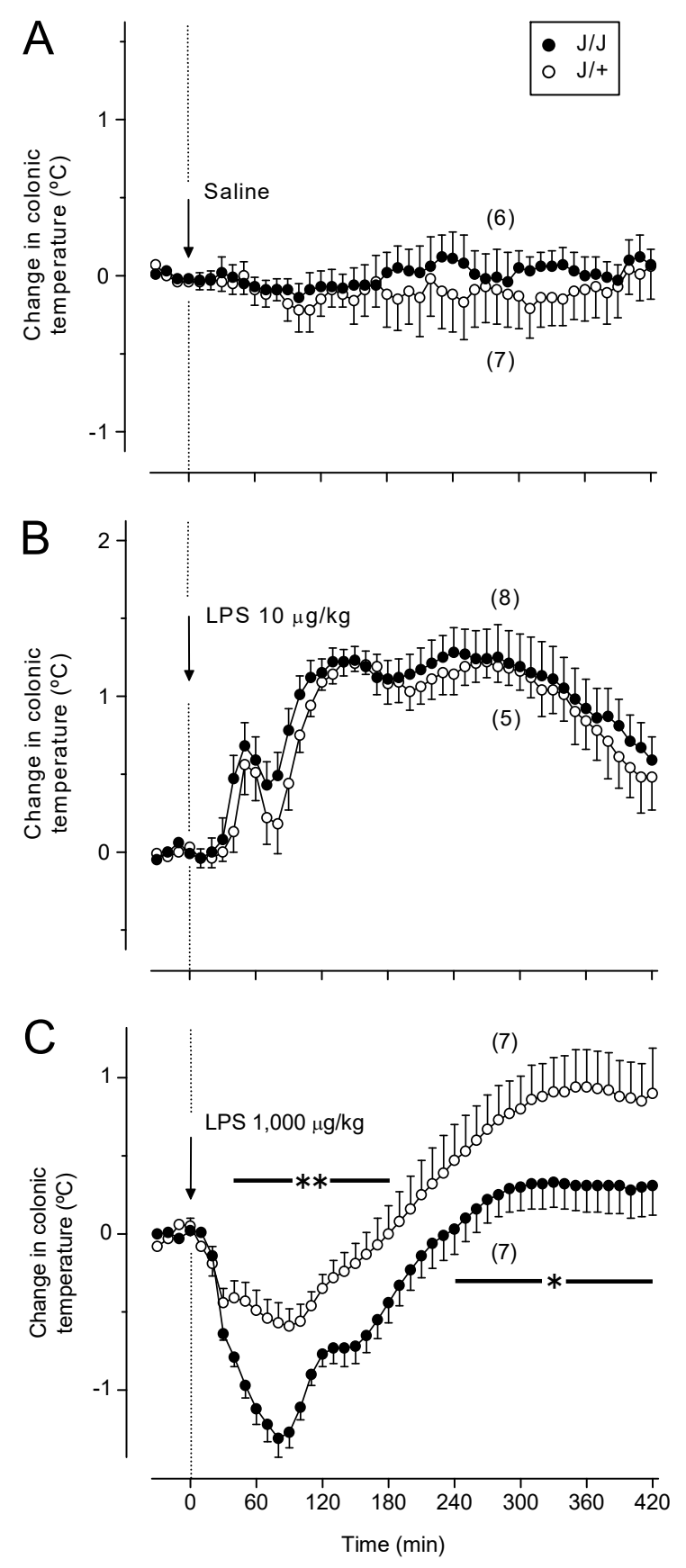

Figure 2. Deep (colonic) $T_{b}$ responses of $J / J$ and $J /+$ rats to the low $(10 \mu \mathrm{g} / \mathrm{kg}$, i.v.) and high $(1,000 \mu \mathrm{g} / \mathrm{kg}$, i.v.) doses of LPS. (A) Administration of the vehicle (saline) does not affect $T_{b}$ in rats. (B) The low dose of LPS causes polyphasic fevers in J/J and $\mathrm{J} /+$ rats, without any significant differences between the genotypes. (C) In J/+ rats, the high dose of LPS induces early hypothermia followed by late fever. In J/J rats, the hypothermic response is exaggerated, and the subsequent febrile response is attenuated, as compared to the J/+ controls. Time periods corresponding to significant intergenotype differences in the response to LPS are marked as $* P<0.05$ or $* * P<0.01$. From Pakai et al. [66]. 


\subsubsection{Renal dysfunction and hepatic damage in Gunn rats after LPS administration}

Next, we studied biomarkers of kidney and liver damage [66]. As markers of renal function, neither blood urea nitrogen (BUN) nor creatinine levels differed significantly between saline-treated $\mathrm{J} / \mathrm{J}$ and $\mathrm{J} /+$ rats. While administration of the low dose of LPS did not raise the renal dysfunction markers, the high dose increased both $\mathrm{BUN}$ and creatinine in $\mathrm{J} / \mathrm{J}(\mathrm{P} \leq 0.001$ for both BUN and creatinine) and $\mathrm{J} /+$ rats $(\mathrm{P}=0.010$ for $\mathrm{BUN} ; \mathrm{P}<0.001$ for creatinine) without any significant differences between the genotypes (Fig. 3).

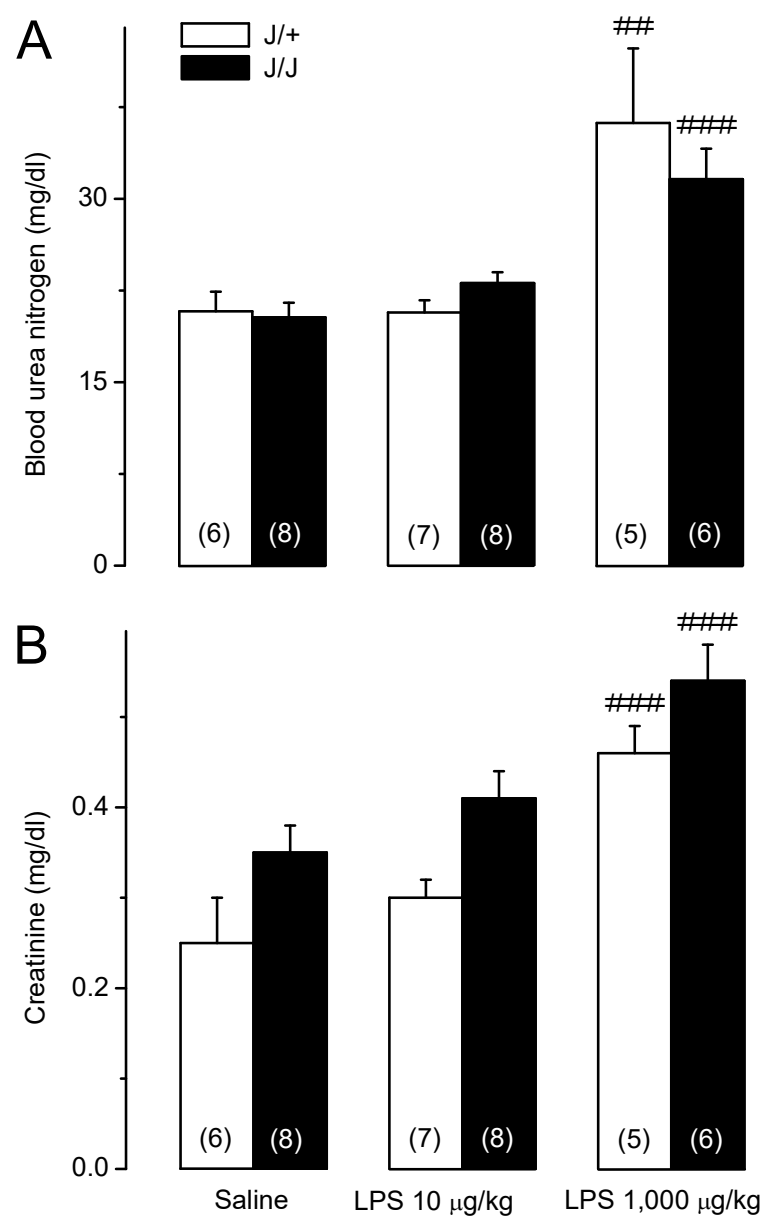

Figure 3. Biochemical markers of renal dysfunction in $J / J$ and $J /+$ rats. (A) Plasma BUN levels do not differ between salinetreated $J / J$ and $J /+$ rats and after administration of the low dose of LPS. The high dose of LPS raises BUN in both genotypes, without any intergenotype difference. (B) Plasma creatinine levels do not differ between saline-treated J/J and J/+ rats and after administration of the low dose of LPS. The high dose of LPS raises plasma creatinine in both genotypes, without any intergenotype difference. Significant differences in the response to LPS compared to saline are marked as ${ }^{\#} P<0.01$ or ${ }^{\# \#} P<0.001$. Number of animals in the corresponding groups are indicated in the figure. From Pakai et al. [66]. 
Plasma alanine aminotransferase (ALT), aspartate aminotransferase (AST), and gamma-glutamyl transferase (GGT) were used to assess hepatocyte damage [72]. Activities of all 3 enzymes were within the normal range in $\mathrm{J} / \mathrm{J}$ and $\mathrm{J} /+$ rats after the injection of saline or the low dose of LPS. When LPS was administered at the high dose, rats showed marked rises in $\operatorname{ALT}(\mathrm{P}=0.019$ for $\mathrm{J} /+$; $\mathrm{P}=0.015$ for $\mathrm{J} / \mathrm{J}$; Fig. 4A), AST $(\mathrm{P}=0.021$ for $\mathrm{J} /+; \mathrm{P}=0.041$ for $\mathrm{J} / \mathrm{J}$; Fig. 4B), and GGT ( $\mathrm{P}<0.001$ for both genotypes; Fig. $4 \mathrm{C})$. In $\mathrm{J} / \mathrm{J}$ rats, the surge was blunted for GGT $(\mathrm{P}=0.042)$ and tended to be reduced for ALT $(\mathrm{P}=0.208)$ and AST $(\mathrm{P}=0.179)$, as compared to $\mathrm{J} /+$ rats.
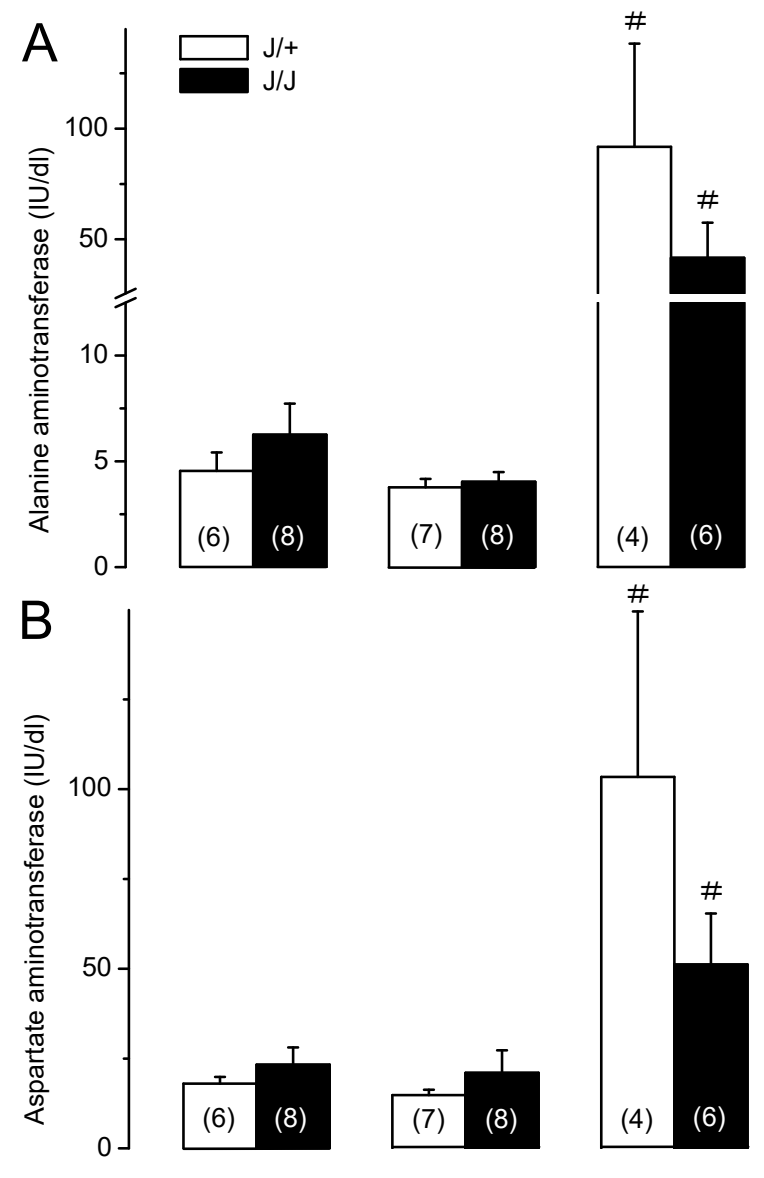

Saline LPS $10 \mu \mathrm{g} / \mathrm{kg} \quad$ LPS $1,000 \mu \mathrm{g} / \mathrm{kg}$ 


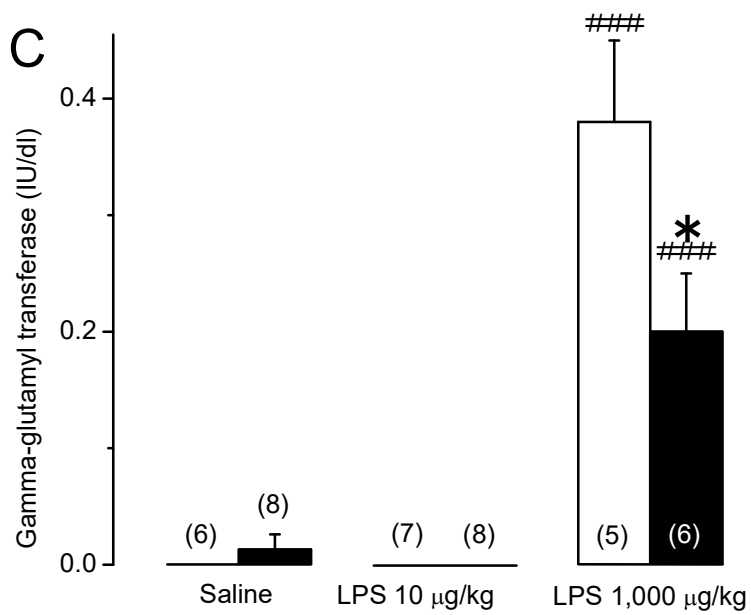

Figure 4. Biochemical markers of hepatocyte damage in J/J and J/+ rats. (A) Plasma ALT levels do not differ between salinetreated $J / J$ and $J /+$ rats and remain unchanged after the administration of the low dose of LPS (10 $\mu \mathrm{g} / \mathrm{kg}$, iv). In response to the high dose of LPS $(1,000 \mu \mathrm{g} / \mathrm{kg}$, iv), plasma ALT surges in both genotypes, but this surge tends to be blunted in J/J rats (as compared to the J/+ controls). (B) Plasma AST levels do not differ between J/J and J/+ rats after administration of saline or LPS at the low dose. In response to the high dose of LPS, AST surges in both genotypes, but the surge tends to be blunted in $\mathrm{J} / \mathrm{J}$ rats). (C) Plasma GGT levels are near the detection threshold in J/J and J/+ rats after administration of saline or the low dose of LPS. Plasma GGT rises in both genotypes in response to the high dose of LPS, but the rise is significantly reduced in $J / J$ rats. A significant $(P<0.05)$ intergenotype difference in the response to LPS is marked with *. Within each genotype, significant differences in the response to LPS (as compared to saline) are marked as ${ }^{\#} P<0.05$ or ${ }^{\# \#} P<0.001$. Number of animals in the corresponding groups are indicated in the figure. From Pakai et al. [66].

4.2. The role of TRPV1 channels in thermoregulation under physiological conditions and in systemic inflammation

\subsubsection{Significance of TRPV1 channels in $T_{b}$ regulation and thermoeffector activities}

In order to determine the role of TRPV1 channel in thermoregulation we studied circadian fluctuations in deep $\mathrm{T}_{\mathrm{b}}$ and thermoeffector activities of a large number of $\operatorname{Tr} p v 1^{+/+}$ and $\operatorname{Trp} v 1^{-/-}$mice in our telemetry and respirometry experimental setups [65]. In both setups the light-phase mean deep $\mathrm{T}_{\mathrm{b}}$ of $\operatorname{Tr} p v 1^{-/-}$mice was slightly, but significantly $(\mathrm{P}<0.05)$ lower than that of the controls. During the dark (active) phase, however, the intergenotype $\mathrm{T}_{\mathrm{b}}$ difference was attenuated in the respirometry setup and completely disappeared in the telemetry setup (Fig 5). As the result of such biphasic dynamics, the daily mean $\mathrm{T}_{\mathrm{b}}$ of $\operatorname{Tr} p v 1^{-/-}$mice was either similar 
to or slightly lower than that of the controls in both setups. We conclude, that these alterations are phase specific and somewhat depend on the experimental setup.
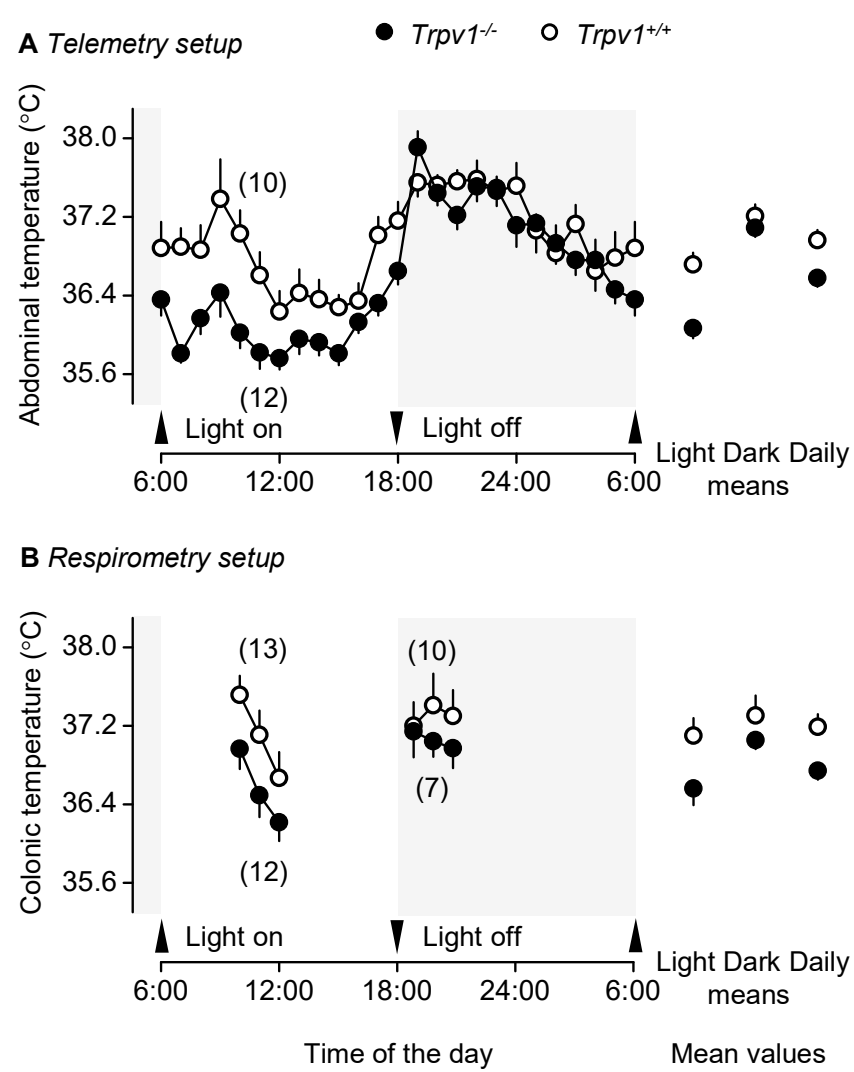

Figure 5. During the inactive (light) phase, Trpv1 $1^{-/}$mice have a lower deep $T_{b}$ than their genetically unaltered counterparts in the telemetry (A), and respirometry (B) setups. Number of animals in the corresponding groups are indicated in the figure. From Garami et al. [65].

Next, we studied two autonomic effectors under basal conditions, namely thermogenesis (measured as $\mathrm{VO}_{2}$ ) and tail skin vasomotor tone (measured as $\mathrm{T}_{\mathrm{sk}}$ ). It has been revealed, that $\operatorname{Tr} p v 1^{-/-}$mice maintained a lower metabolic rate and showed a more pronounced cutaneous vasoconstriction than their $\operatorname{Tr} p v 1^{+/+}$counterparts, regardless of the phase (Fig. 6). The daily means for $\mathrm{VO}_{2}$ and $\mathrm{T}_{\mathrm{sk}}$ for the $\mathrm{KO}$ versus control mice were $18.9 \pm 1.0$ versus $26.3 \pm 1.3$ $\mathrm{ml} / \mathrm{kg} / \mathrm{min}(\mathrm{p}<0.0001)$ and $35.0 \pm 0.2$ versus $35.7 \pm 0.2^{\circ} \mathrm{C}(\mathrm{p}<0.05)$, respectively.

In summary, Trpv1/- mice have a thermoregulatory phenotype that shows a specific thermoeffector pattern: these mice are are hypometabolic, and vasoconstricted while maintain 
lower $\mathrm{T}_{\mathrm{b}}$ as compared to control mice, therefore this animal model is suitable to investigate the role of TRPV1 in systemic inflammation.

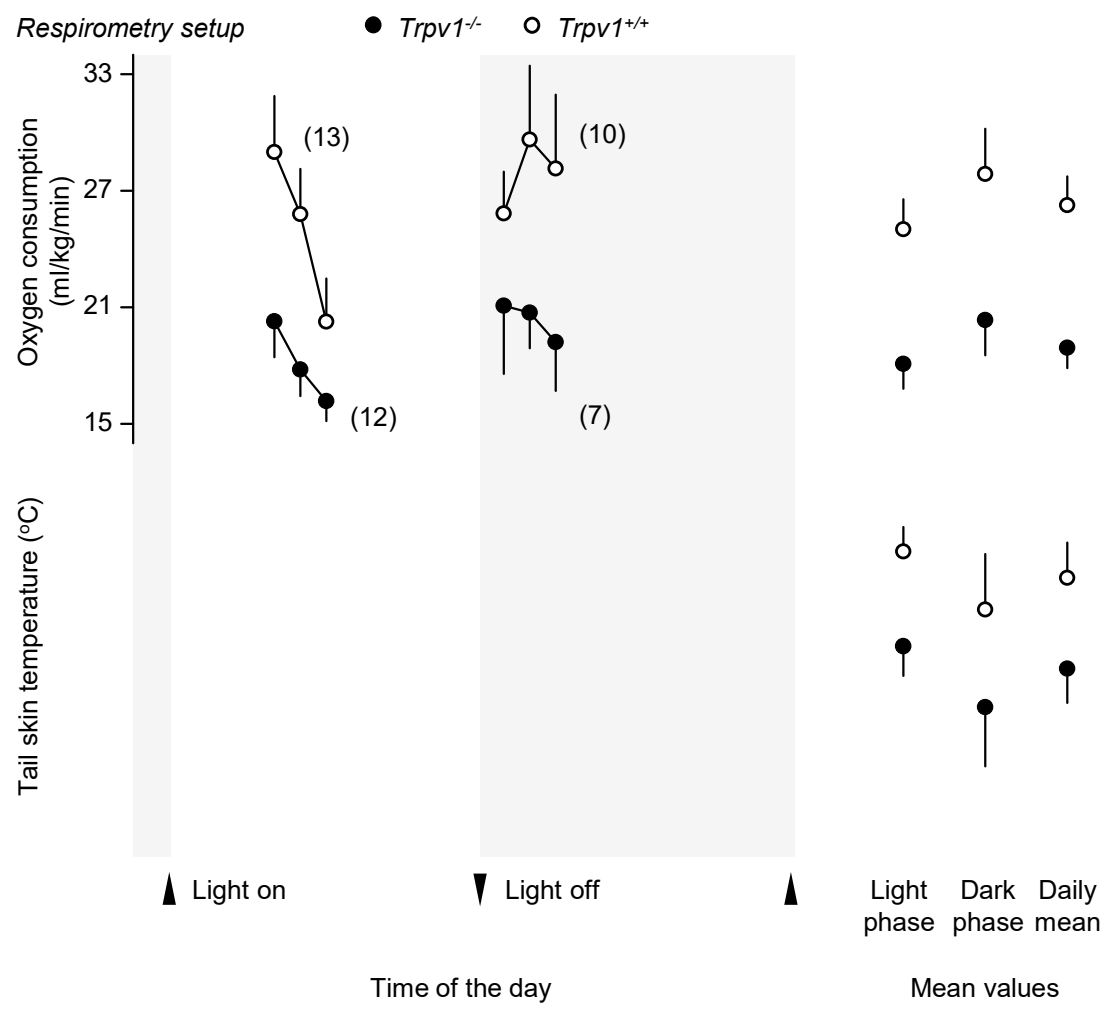

Figure 6. Compared to genetically unaltered controls, Trpv $1^{-/}$mice regulate $T_{b}$ by using a different thermoeffector pattern. In the respirometry setup, they are hypometabolic (have a lower $\mathrm{VO}_{2}$ ) and vasoconstricted (have a lower tail $T_{s k}$ ). Number of animals in the corresponding groups are indicated in the figure. From Garami et al. [65].

\subsubsection{The role of TRPV1 channels in aseptic or microbial sepsis}

In the laboratory, systemic administration of LPS in rodents is often used to induce SIRS aseptically, while polymicrobial sepsis is often studied in mice and rats subjected to CLP procedure. To discover the role of TRPV1 channel in systemic inflammation, we investigated the effect of LPS and CLP in mice either present or missing the channel [68].

Young adult mice responded to LPS (40 mg/kg, i.p.) with a marked, rapidly progressing SIRS (Fig. 7A). Pretreatment with AMG517 (210 $\mu \mathrm{g} / \mathrm{kg}$, s.c.), a potent and selective TRPV1 antagonist, profoundly decreased the survival rate at multiple time points (e.g., from $50 \%$ to 
$5 \%$ at $26 \mathrm{~h}, \mathrm{P}<0.001$ ) and also shortened the mean time to death (from $26 \pm 2$ to $19 \pm 1 \mathrm{~h}, \mathrm{P}=0.003$ ). All LPS-treated mice within the present study developed profound hypothermia (decrease from $\sim 36^{\circ} \mathrm{C}$ to $\sim 33^{\circ} \mathrm{C}$ at $10 \mathrm{~h}$ ). Similar to our previous studies $[73,74]$ in this study AMG517 caused a short-lasting increase in $\mathrm{T}_{\mathrm{b}}$ compared with the vehicle $(\mathrm{P}<0.01$, Fig. $7 \mathrm{~B})$, thus confirming an effective systemic blockade of TRPV1 channels. Overall, the results of our experiment show that pharmacological blockade of TRPV1 increases mortality of young mice in LPS-induced SIRS.
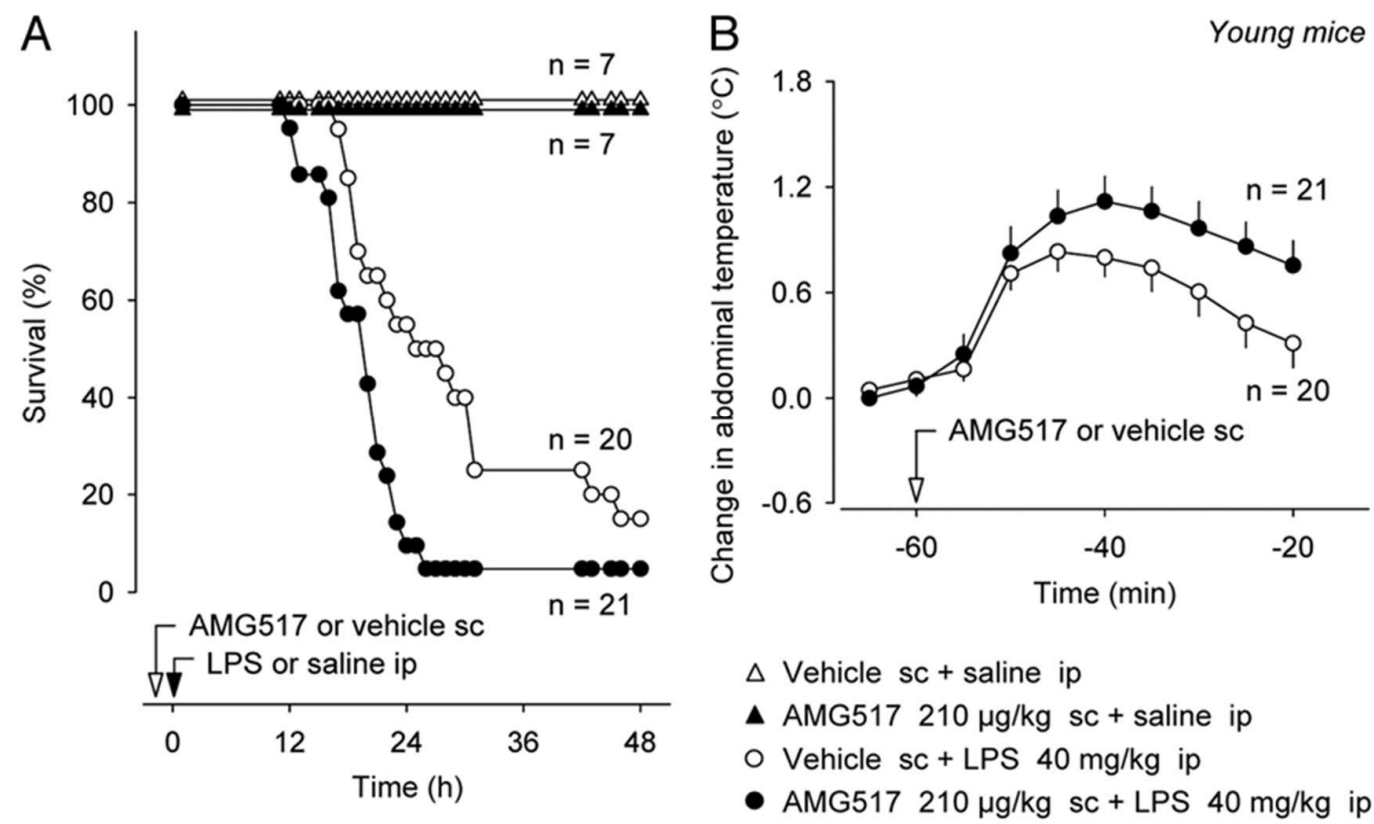

Figure 7. Systemic pretreatment with AMG517 (210 $\mu \mathrm{g} / \mathrm{kg}$, s.c.) decreases survival of young mice in LPS-induced SIRS (A). Confirming an effective blockade of TRPV1 channels, the AMG517 pretreatment increases deep Tb in young mice (B). From Wanner et al. [68].

The outcome of LPS-induced SIRS in older mice was more severe than in young mice. Pretreatment of aged mice with AMG517 increased the survival rate, delayed the mean time to death and decreased the risk of mortality, which effects are directly the opposite of those observed in the young mice (figure not shown).

Then, we tested whether genetic deletion of TRPV1 would have the same effects on SIRS in middle-aged mice as a pharmacological blockade. Experiments were conducted in 43- 
44-week-old Trpv1 $1^{-/-}$mice of both sexes and in their age- and sex-matched $\operatorname{Tr} p v 1^{+/+}$controls. LPS caused death in all wild-type control mice but only in $80 \%$ of $\operatorname{Tr} p v 1^{-/-}$mice. Survival rate of $\operatorname{Trp} v 1^{-/-}$mice was 2-3 times higher at certain time points than of $\operatorname{Tr} p v 1^{-/-}$mice. Genetic deletion of TRPV1 channels decreased the risk of mortality and tended to delay death. Importantly, $\operatorname{Trp} v 1^{-/-}$mice exhibited a 70\% suppression of the TNF- $\alpha$ response at $12 \mathrm{~h}$ postLPS (figure not shown).

After these results, we investigated whether the attenuation of aseptic SIRS observed in older $\operatorname{Trpv} 1^{-/-}$mice would result in attenuation of the body's defense against CLP-induced polymicrobial infection. We observed that CLP sepsis caused substantial mortality in aged mice of both genotypes (Fig. 8A), however, $\operatorname{Tr} p v 1^{-/-}$mice died significantly faster than their $\operatorname{Tr} p v 1^{+/+}$ controls. The mean time to death in $\operatorname{Trp} v 1^{-/-}$mice was $20 \pm 2 \mathrm{~h}$, compared to $52 \pm 11 \mathrm{~h}$ in $\operatorname{Trp} v 1^{+/+}$ mice $(\mathrm{P}<0.01)$, and the survival rate in $\operatorname{Trp} v 1^{+/+}$mice was 3.9 times higher than in $\operatorname{Trp} v 1^{-/-}$mice $(86 \%$ vs. $22 \%, \mathrm{P}<0.001)$. The delayed recovery of deep $\mathrm{T}_{\mathrm{b}}$ after the CLP procedure also confirms the higher severity of sepsis in $\operatorname{Trp} v 1^{-/-}$mice $(\mathrm{P}<0.001$, Fig. 4B).
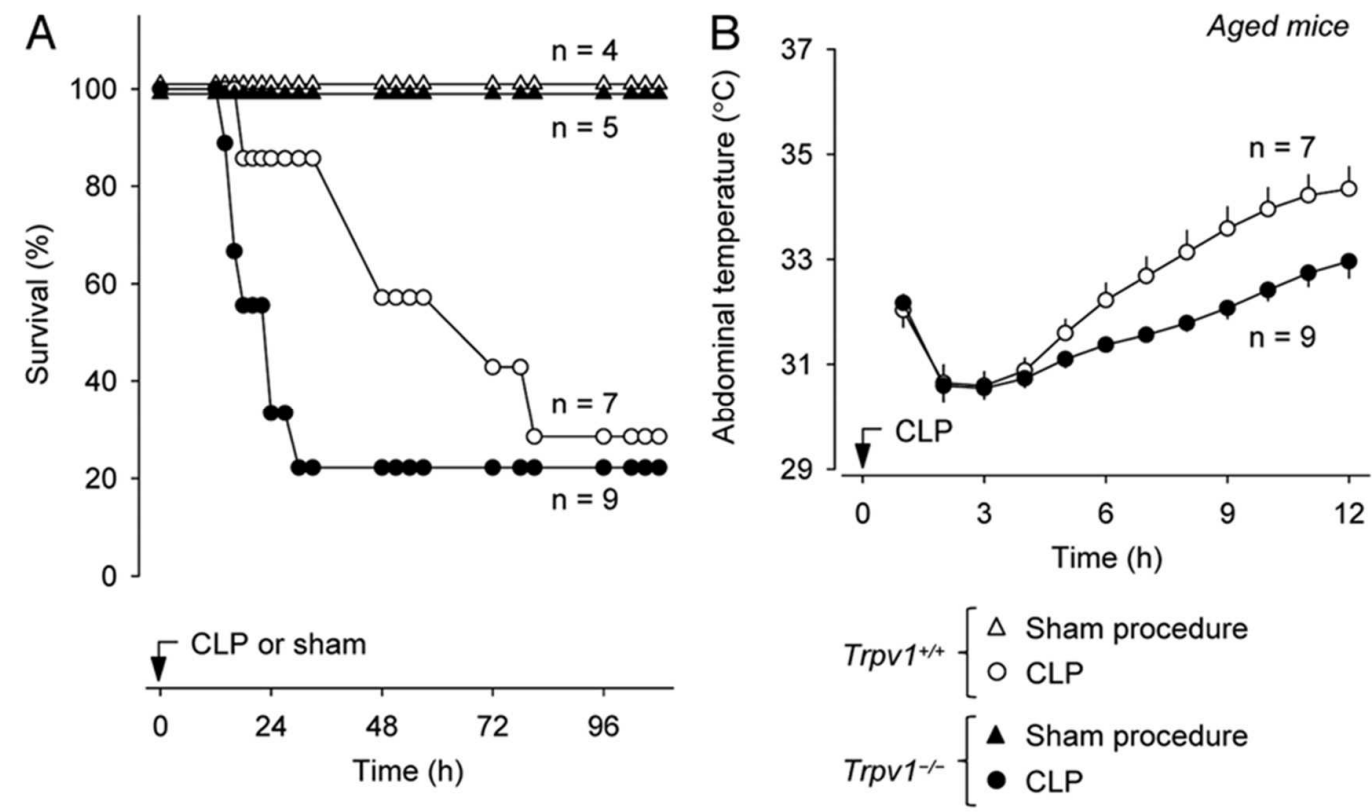

Figure 8. Compared with their age-matched wild-type littermates, middle-aged Trpv1 ${ }^{-1}$ mice have a shorter survival time (A) and a slower $T_{b}$ recovery (B) during CLP-induced sepsis. From Wanner et al. [68]. 


\subsection{Characterization of the thermoregulatory effects of PACAP}

4.3.1. Aspects of the thermoregulatory response to central and systemic PACAP38 administration in rats

To identify the thermoregulatory effect of PACAP38 in details, we infused 10 or 100 $\mu \mathrm{g} / \mathrm{kg}$ of the peptide (or saline) into the lateral cerebral ventricle of rats and recorded their $\mathrm{T}_{\mathrm{c}}$, $\mathrm{T}_{\mathrm{sk}}$, and $\mathrm{VO}_{2}$ in our respirometry setup [64]. Both of the applied doses of PACAP38 caused a marked rise in the $T_{c}$ starting already at 10 min after the injection $(\mathrm{P}<0.001$ for both; Fig. 9). The magnitude of the PACAP38-induced hyperthermia was dose-dependent with a maximal $\mathrm{T}_{\mathrm{c}}$ change of $2.0 \pm 0.3^{\circ} \mathrm{C}$ and $1.4 \pm 0.3^{\circ} \mathrm{C}$ at the dose of 100 and $10 \mu \mathrm{g} / \mathrm{kg}$, respectively $(\mathrm{P}<0.001$ for both). Significant difference $(\mathrm{P}<0.001)$ could be statistically confirmed also between the effects of the $10 \mathrm{vs} .100 \mu \mathrm{g} / \mathrm{kg}$ dose of PACAP38 on $\mathrm{T}_{\mathrm{c}}$.

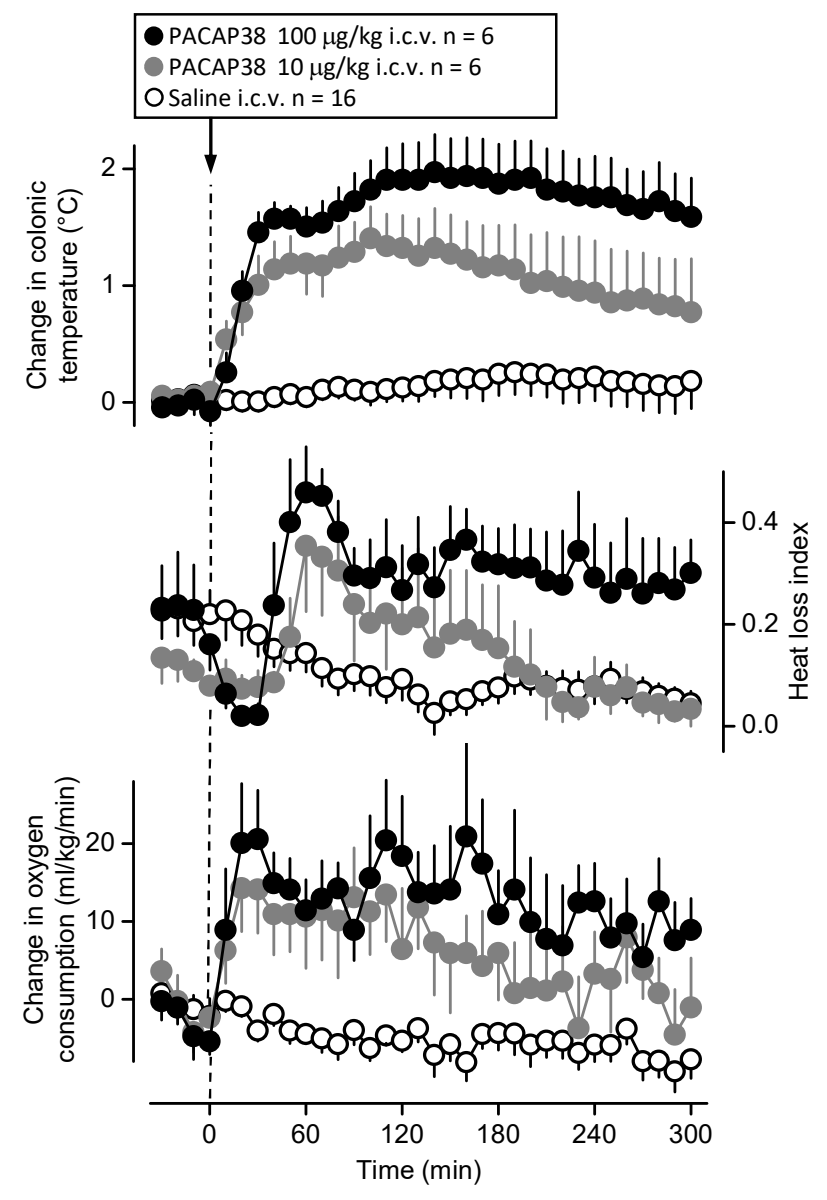

Figure 9. Thermoeffector and $T_{c}$ responses of rats to different doses (10 and $100 \mu \mathrm{g} / \mathrm{kg}$ ) of PACAP38 or saline administered i.c.v. The changes of $T_{c}$ (a measure of deep $T_{b}$ ) are shown in the upper panel; alterations in the activity of the two main autonomic thermoeffectors, $\mathrm{HLI}$ and $\mathrm{VO}_{2}$, are plotted in the middle and lower panel, respectively. These experiments were performed in the respirometry setup at a $T_{a}$ of $28^{\circ} \mathrm{C}$. From Banki et al. [64]. 
The increased $T_{b}$ response to central administration of PACAP38 is in harmony with earlier reports in rats $[57,58,75,76]$. In the case of the higher dose, the development of the hyperthermia was accompanied by significant tail skin vasoconstriction [as indicated by a decreased heat loss index (HLI); $\mathrm{P}<0.05$ ]. It is a novel finding to report cutaneous vasomotor responses to i.c.v. PACAP38 in conscious rats. The presence of the vasomotor response indicates that the thermoregulatory (constrictor) effect of PACAP38 on the cutaneous vascular tone is different from its direct (dilator) effect on skin vessels, which was shown earlier in small rodents $[77,78]$. The initial drop of HLI lasted for $\sim 40 \mathrm{~min}$, and then, it was followed by a marked elevation of HLI due to tail skin vasodilation, which remained significantly $(\mathrm{P}<0.05)$ higher than the HLI of saline-treated rats until the end of the experiment. Similarly to the $T_{c}$ response, the $\mathrm{VO}_{2}$ of the rats increased already at 10 min after administration the peptide i.c.v. as compared to control animals in a dose-dependent manner. It reached a maximal rise of $21 \pm 6$ and $14 \pm 6 \mathrm{ml} / \mathrm{kg} / \mathrm{min}$ at 100 and $10 \mu \mathrm{g} / \mathrm{kg}$, respectively $(\mathrm{P}<0.001$ for both). This finding is in harmony with previous studies, in which PACAP38 increased the metabolic rate $[57,79,80]$. In addition, our results demonstrate that simultaneous immediate activation of both autonomic cold-defense thermoeffectors [cutaneous vasoconstriction and brown adipose tissue (BAT) thermogenesis] contribute to the development of hyperthermia in response to PACAP38.

To consider the possibility of a peripheral site of action for PACAP38 in a comparative experiment, we looked into whether the higher dose of PACAP38 $(100 \mu \mathrm{g} / \mathrm{kg})$ that caused pronounced hyperthermia when injected centrally has a similar effect on deep $T_{b}$ in the case of a systemic administration. When PACAP38 at $100 \mu \mathrm{g} / \mathrm{kg}$ was infused i.v., it caused a slight, but significant $(\mathrm{P}<0.05)$ rise of $\mathrm{T}_{\mathrm{c}}$. Both the maximum $\mathrm{T}_{\mathrm{c}}$ elevation and the duration of the hyperthermic effect to systemic PACAP38 infusion were substantially less than what i.c.v. administration of the same dose evoked $(\mathrm{P}<0.05)$. Similarly to what we observed after i.c.v. drug delivery, after i.v. administration the hyperthermia also developed due to decreased heat loss and increased $\mathrm{VO}_{2}$, but all parameters changed to a much lesser extent compared to i.c.v. injection (figure not shown). Our results certainly show that the site of action for the thermoregulatory response to PACAP38 is situated within the CNS. 


\subsubsection{Characteristics of the thermoregulatory response in the absence of PACAP}

After characterization of the thermoregulatory response to exogenous PACAP38 administration, we studied how deep $\mathrm{T}_{\mathrm{b}}$ is affected by the absence of PACAP [64].

For that, we analyzed the circadian changes of $\mathrm{T}_{\mathrm{ab}}$ and locomotor activity in freely moving Pacap ${ }^{-/-}$and Pacap ${ }^{+/}$mice (Fig. 10A). Mice of both genotypes had lower $\mathrm{T}_{\mathrm{ab}}$ and activity levels during the inactive (light) phase compared to the active (dark) phase, however Pacap $^{-/-}$mice were more active than their wild-type littermates during both phases of the day $(\mathrm{P}<0.001)$. During most of the light phase, the increased locomotor activity resulted in a slightly higher $\mathrm{T}_{\mathrm{ab}}$ in the $\mathrm{KO}$ mice compared to controls $(\mathrm{P}<0.05)$; but in the night, there was no significant difference in $\mathrm{T}_{\mathrm{ab}}$ between the groups. It can be hypothesized that the different lightdark influence of locomotor activity on deep $\mathrm{T}_{\mathrm{b}}$ can originate from the circadian changes of cutaneous vasodilation, thus heat loss mechanisms [81].

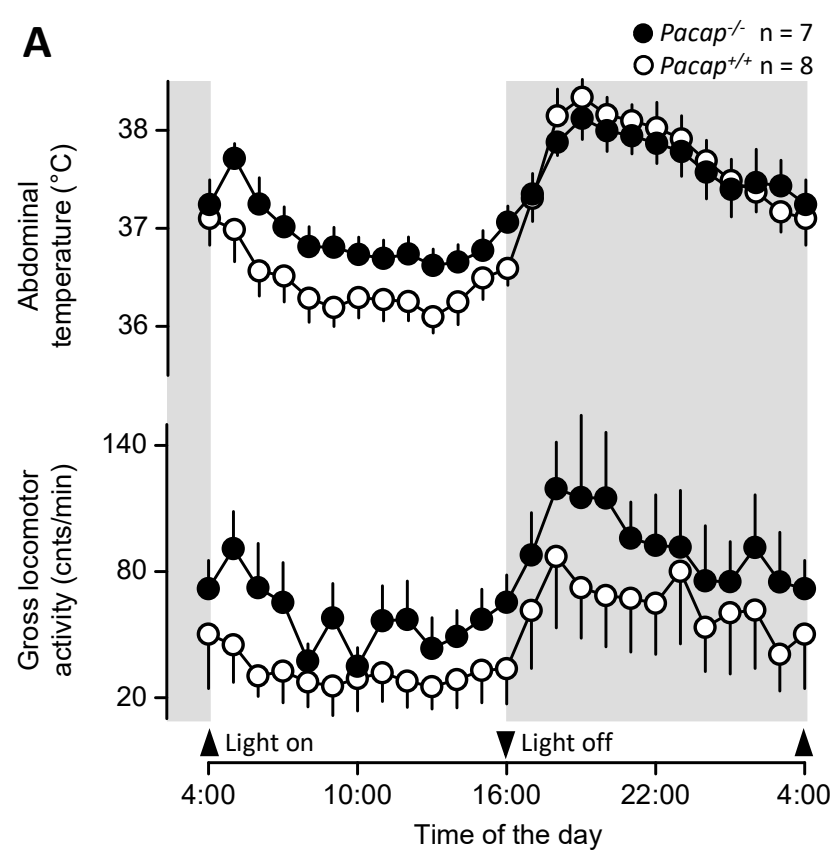



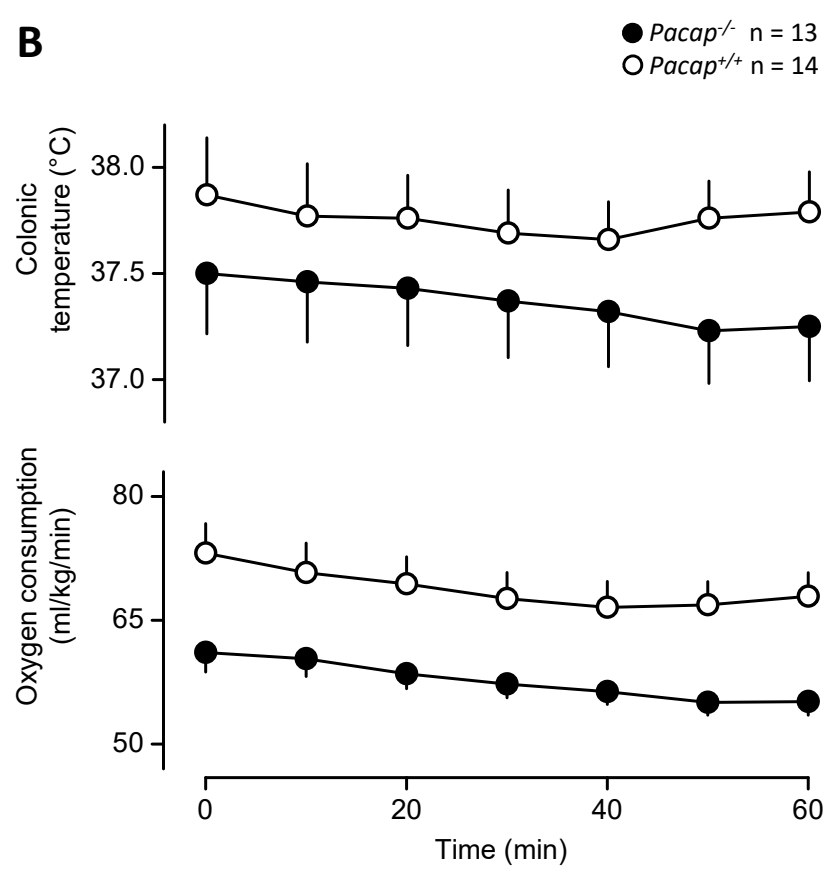

Figure 10. Circadian changes of $T_{a b}$ and locomotor activity in freely moving Pacap ${ }^{-/}$and Pacap ${ }^{+/+}$mice. These experiments were performed in the telemetry setup at a $T_{a}$ of $27^{\circ} \mathrm{C}(\mathrm{A})$. Basal $T_{c}$ and $\mathrm{VO}_{2}$ of loosely restrained Pacap ${ }^{-/}$and Pacap ${ }^{+/+}$mice. Recordings of $T_{c}$ and $\mathrm{VO}_{2}$ were performed in the respirometry setup at a $T_{a}$ of $31^{\circ} \mathrm{C}(B)$. From Banki et al. [64].

Then, we measured the basal (resting) $\mathrm{T}_{\mathrm{c}}$ and $\mathrm{VO}_{2}$ in loosely restrained Pacap ${ }^{-/}$and Pacap $^{+/+}$mice in our respirometry setup (Fig. 10B). We recorded the basal thermoregulatory parameters for $60 \mathrm{~min}$ starting from $11 \mathrm{a} . \mathrm{m}$. because this time period corresponded to the biggest difference in deep $\mathrm{T}_{\mathrm{b}}$ between freely moving Pacap $^{-/}$and Pacap $^{+/+}$mice (Fig. 10A). As shown in Figure 10B, the basal $\mathrm{VO}_{2}$ appeared to be significantly lower in Pacap ${ }^{-/}$mice as compared to controls throughout the experiment $(\mathrm{P}<0.001)$. In correlation with their hypometabolism, $\mathrm{T}_{\mathrm{c}}$ of the Pacap $^{-/-}$mice was also slightly lower than that of controls $(\mathrm{P}<0.01)$.

Finally, to determine which neurons are responsible for maintaining the reduced resting metabolic rate in $\mathrm{Pacap}^{-/-}$mice, we measured expression of the inducible transcription factor cFos, a marker of neuronal activation [82], in the MnPO and MPO (Fig. 11A). It is well known from previous studies, that neurons in these brain areas are involved in the regulation of thermogenesis $[47,83]$. In the MnPO, we have not found statistical difference in the number of c-Fos-positive cells between genotypes; however, c-Fos expression was nearly three times higher $(\mathrm{p}<0.05)$ in the MPO of the Pacap ${ }^{-/}$mice as compared to controls (Fig. 11B). 
It has been revealed that gamma-aminobutyric acid (GABA)ergic neurons in the MPO tonically suppress BAT thermogenesis [84] and are believed as the first effector neurons of the thermoregulatory loops controlling autonomic thermoeffectors [47]. Therefore, our findings in Pacap $^{-/-}$mice suggest that the absence of PACAP results in an increased activation of the inhibitory MPO neurons leading to more pronounced suppression of thermogenesis.
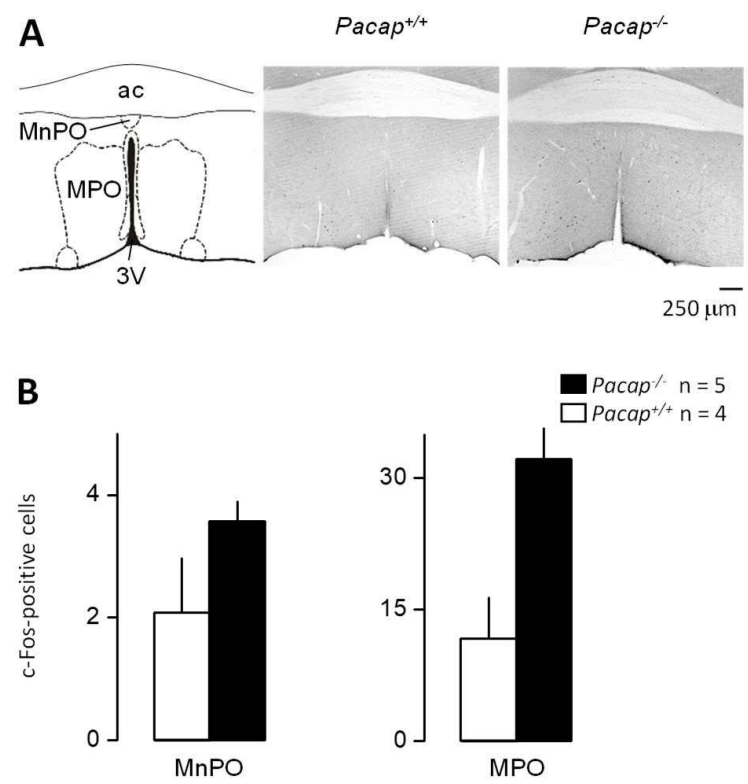

Figure 11. The expression of c-Fos positive cells in the MnPO and MPO of Pacap ${ }^{-/}$and Pacap ${ }^{+/+}$mice (A). Quantitative analyses of c-Fos immunoreactive cells in the MnPO and MPO (B). From Banki et al. [64].

4.4. The role of the NK1 receptor in systemic inflammatory processes

4.4.1. Characteristics of the thermoregulatory response of Tacr $1^{+/+}$and Tacr ${ }^{-/}$mice to $L P S$

Neurokinin signaling is thought to be involved in various inflammatory processes. To identify how the NK1 receptor contributes to the febrile response, we first determined the thermoregulatory phenotype of mice with the gene encoding the NK1 receptor either present $\left(\right.$ Tacr $\left.^{+/+}\right)$or absent $\left(\right.$Tacr $\left.^{-/-}\right)$[67]. The basal deep $\mathrm{T}_{\mathrm{b}}$ of the mice was identical regardless of either gender or genotype in the thermocouple setup throughout the recordings. To evaluate whether $\mathrm{Tacrl}^{-/-}$mice are able to appropriately activate warmth- and cold-defense mechanisms, we studied the thermoregulatory response of these mice to ambient heating and cooling, 
respectively. It could be concluded, that the extent and dynamics of $T_{b}$ or $T_{s k}$ response did not differ between the two groups, hence, the thermoregulatory response of $\mathrm{Tacr}^{-/-}$mice to heat or cold exposure is unaltered compared to controls (figure not shown).

In our thermophysiological experiments, when treated with LPS $(120 \mu \mathrm{g} / \mathrm{kg}$, i.p. $)$, the mice of both genotypes developed fever as compared to saline-treated groups (Fig. 12A, B).

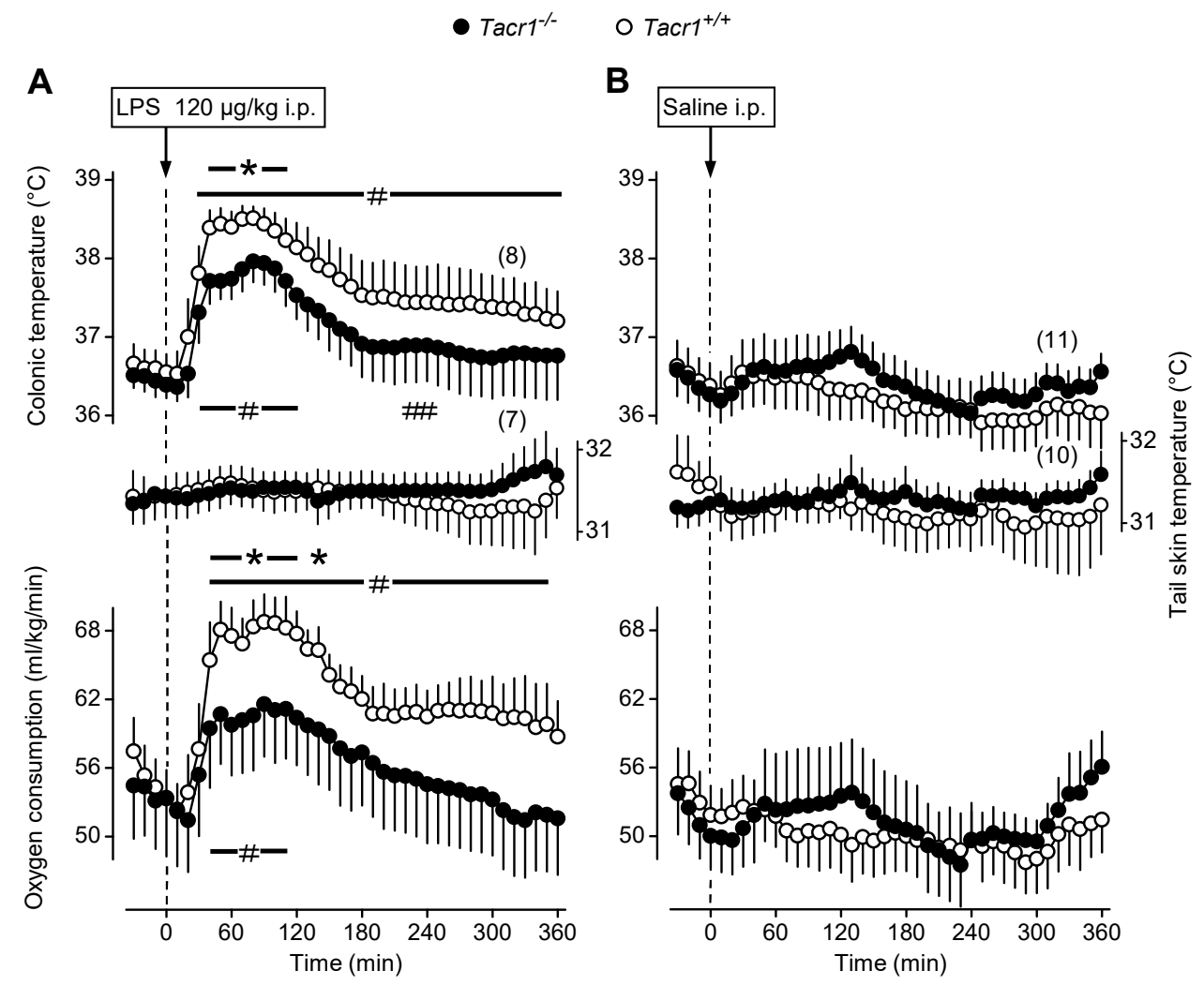

Figure 12. Thermoeffector and $T_{c}$ responses of Tacr $1^{+/+}$and Tacr $1^{-/}$mice to LPS (A) or saline (B) administered i.p. These experiments were performed in the respirometry setup at an $T_{a}$ of $31{ }^{\circ} \mathrm{C}$. Number of animals in the corresponding groups are indicated in the figure. ${ }^{*} P<0.05$, intergenotype difference in the response to LPS. ${ }^{*} P<0.05$, LPS vs. saline difference within the same genotype. From Pakai et al. [67].

LPS-treated Tacr $^{+/+}$mice responded with a typical fever response: their deep $\mathrm{T}_{\mathrm{b}}$ started to increase at $20 \mathrm{~min}$, reached plateau $\left(\sim 38.5^{\circ} \mathrm{C}\right)$ between 40 and $100 \mathrm{~min}$, then it gradually decreased, but remained elevated compared to saline treatment throughout the experiment ( $\mathrm{P}<0.05$ at 30-360 min). In Tacr $1^{-/-}$mice, the LPS-induced fever response was less pronounced, 
reaching the level of significance at 40-140 min compared to controls $(\mathrm{P}<0.05$; Fig. 12A). The LPS-induced increase in deep $\mathrm{T}_{\mathrm{b}}$ was generated by an elevation of $\mathrm{VO}_{2}$, changing with parallel dynamics as $\mathrm{T}_{\mathrm{b}}$ in both genotypes (Fig. 12A, B). After LPS injection, the $\mathrm{VO}_{2}$ was significantly higher compared to saline treatment at 40-350 min in $\operatorname{Tacr}^{+/+}$mice $(\mathrm{P}<0.05)$ and at 40-110 min in Tacrl $^{-/-}$mice $(\mathrm{P}<0.05)$. Since the experiments were performed at the lower end of the thermoneutral zone (TNZ), the mice showed cutaneous vasoconstriction (as indicated by their low tail $\mathrm{T}_{\mathrm{sk}}$ ), thus no further decrease in tail $\mathrm{T}_{\mathrm{sk}}$ was observed.

Importantly, in Tacr $^{-/-}$mice the LPS-induced elevation of the $\mathrm{T}_{\mathrm{b}}$ and $\mathrm{VO}_{2}$ were markedly suppressed compared to their $\mathrm{Tacr}^{+/+}$littermates starting from 40 min post-LPS infusion until the end of the experiment; both parameters were significantly attenuated at 40$120 \min (\mathrm{P}<0.05$ for intergenotype difference). Our results demonstrate that LPS-induced fever is attenuated in $\mathrm{Tacr} 1^{-/-}$mice already in the early stage.

4.4.2. LPS-induced changes in serum cytokines levels and COX-2 expression in Tacrl $^{+/+}$and Tacrl $^{-/-}$mice

Next, we wanted to know the suppression of which part of the "pyrogenic cytokineCOX-2-PGE 2 " axis is responsible for attenuating the fever response in the absence of Tacr 1 gene [67].

In response to $\mathrm{PGE}_{2}$ administered i.c.v., in the mice of both genotypes a marked elevation in deep $\mathrm{T}_{\mathrm{b}}$ and $\mathrm{VO}_{2}$ rapidly developed, but there was no attenuation in either the $\mathrm{T}_{\mathrm{b}}$ or $\mathrm{VO}_{2}$ rise in the Tacrl $^{-/-}$mice compared to their Tacrl ${ }^{+/+}$littermates (figure not shown). The lack of attenuation in the $\mathrm{PGE}_{2}$-induced thermoregulatory response of the $\mathrm{Tacr} \mathrm{I}^{-/}$mice suggests that their reduced fever response to LPS is due to suppression of an earlier phase in the febrigenic molecular pathway.

One of the early steps in LPS-induced fever signaling is the activation of immune cells, which causes augmented production of inflammatory cytokines, including TNF- $\alpha$ and IL-6. Thus, in our next experiments we measured serum concentrations of these cytokines in the $\mathrm{TaCrl}^{+/+}$and $\mathrm{Tacrl}^{-/-}$mice to determine whether the LPS-induced cytokine production is suppressed in the absence of the NK1 receptor. The administration of LPS resulted in a massive rise of TNF- $\alpha\left(\mathrm{P}<0.001\right.$ vs. saline for both genotypes) and IL-6 $\left(\mathrm{P}<0.01\right.$ for Tacr ${ }^{+/+}$mice and 
$\mathrm{P}<0.001$ for Tacrl $^{-/-}$mice) (figure not shown). We did not find any significant intergenotype difference in the TNF- $\alpha$ and IL-6 levels. These data indicate that impaired pyrogenic cytokine production does not contribute to the attenuated fever response of $\mathrm{Tacr}^{-/-}$mice to LPS.

We moved downstream in the fever signaling pathway and compared the LPS-induced expression of COX-2 between Tacrl $^{+/+}$and Tacr $1^{-/-}$mice. At the mRNA level, COX-2 expression is upregulated in rats already in the early phase of the febrile response $(\sim 30-40 \mathrm{~min}$ after LPS infusion) both in peripheral organs (lungs and liver) and to a lesser extent in the brain [85]. As our present study revealed that the fever response of $T a c r 1^{-/-}$mice was attenuated already in the early phase post-LPS infusion, we collected lung, liver, and brain samples at $\sim 40$ min time point and studied the COX-2 mRNA expression in these tissues. The administration of LPS caused transcriptional upregulation of COX-2 mRNA in the lungs (Fig. 13A), in the liver (Fig. 13B), and in the brain (Fig. 13C) of both genotypes ( $\mathrm{P}<0.001 \mathrm{vs}$. saline for all three tissues) without any significant intergenotype difference. The magnitude of the LPS-induced increase in the level of COX-2 expression was diverse in the three organs studied: $\sim 5$-fold in the lungs, 17-20-fold in the liver, and 2-fold in the brain. These results are well in accordance with earlier findings on the dynamics of LPS-induced COX-2 mRNA expression, but cannot explain the attenuation of the febrile response in the $\mathrm{Tacrl}^{-/-}$mice.
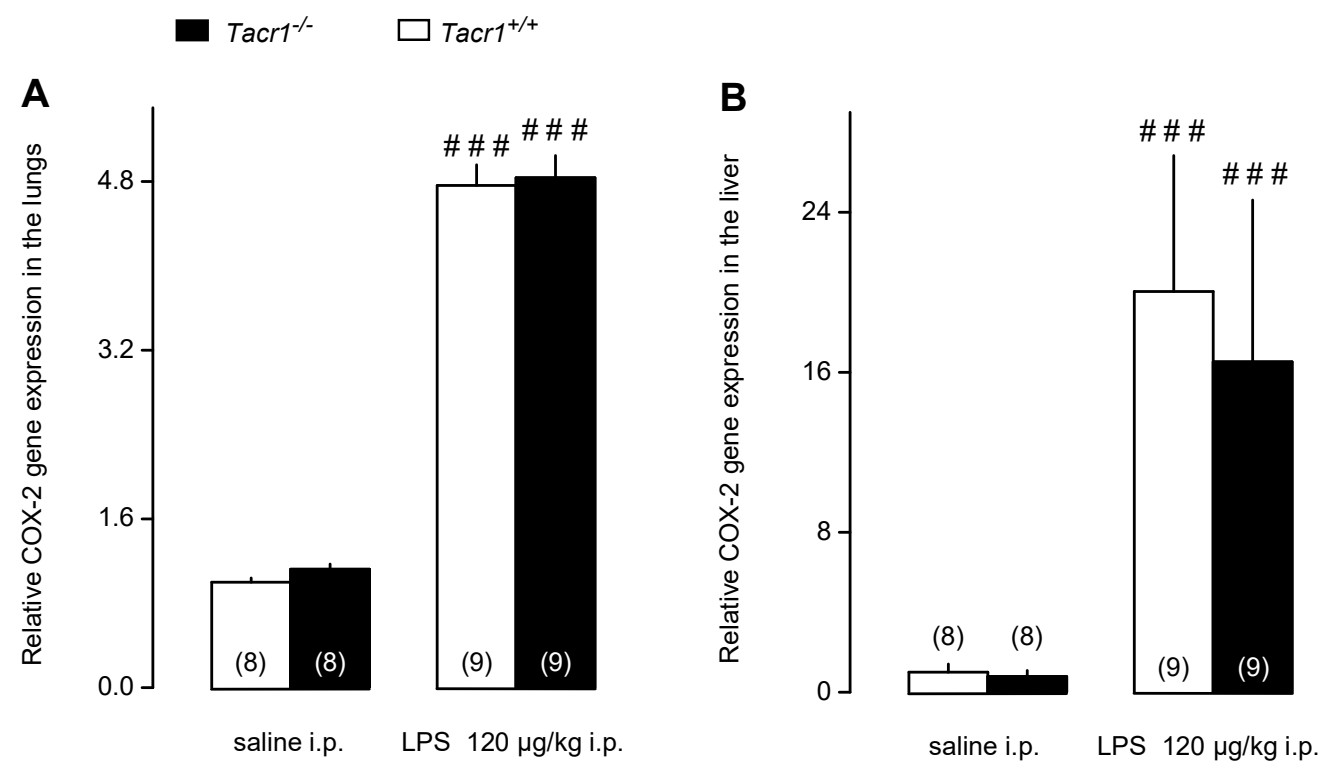


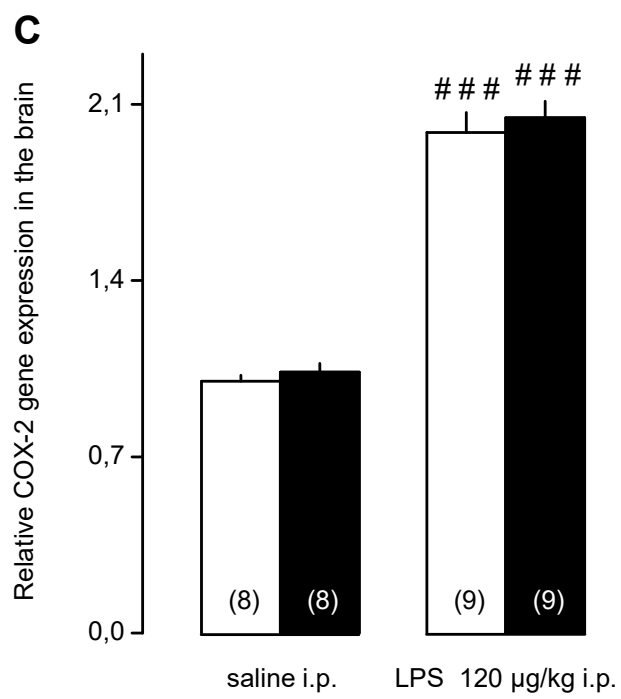

Figure 13. Relative COX-2 gene expression in the lungs (A), liver (B), and in the brain (C) of Tacr $1^{+/+}$and Tacr $1^{-/-}$mice after infusion of LPS or saline. Tissue samples were collected at 40 min postinfusion. Number of animals in the corresponding groups are indicated in the figure. Significant differences in the response to LPS compared to saline are marked as ${ }^{\# \# P<0.001 .}$ From Pakai et al. [67].

Besides the LPS-induced amplified expression of COX-2 mRNA, the expression of the COX-2 protein was also described to increase in the periphery (but not in the brain) already in the early phase of the febrile response [86]. Therefore, we also analyzed the COX-2 protein expression between LPS-treated TacrI $^{+/+}$and Tacr $^{-/-}$mice (Fig. 14).

In $\operatorname{Tacr}^{+/+}$mice, the administration of LPS resulted in a marked increase in COX-2 protein expression in the lungs $(\mathrm{P}<0.01$ vs. saline) (Fig. 14A) and in the liver $(\mathrm{P}<0.05)$ (Fig. 14B), but not in the brain ( $\mathrm{P}=0.264$ ) (Fig. 14C). In LPS-treated Tacr $1^{-/-}$mice, the COX-2 protein expression did not change significantly in either the lungs or the liver compared to saline treatment. In LPS-treated mice, the COX-2 protein expression was attenuated in the lungs of Tacr $1^{-/-}$mice compared with their controls presenting the gene $(\mathrm{P}<0.01)$, while in the liver only a tendency could be seen for reduced COX-2 expression in Tacr $1^{-/-}$mice $(\mathrm{P}=0.101)$. These findings indicate that the absence of the NK1 receptor interferes with the increase of COX-2 expression at the protein level. 


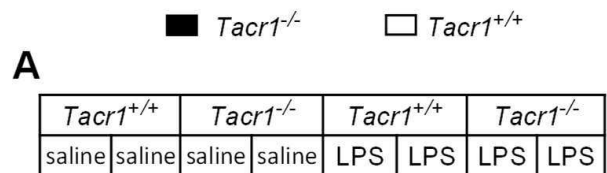

B
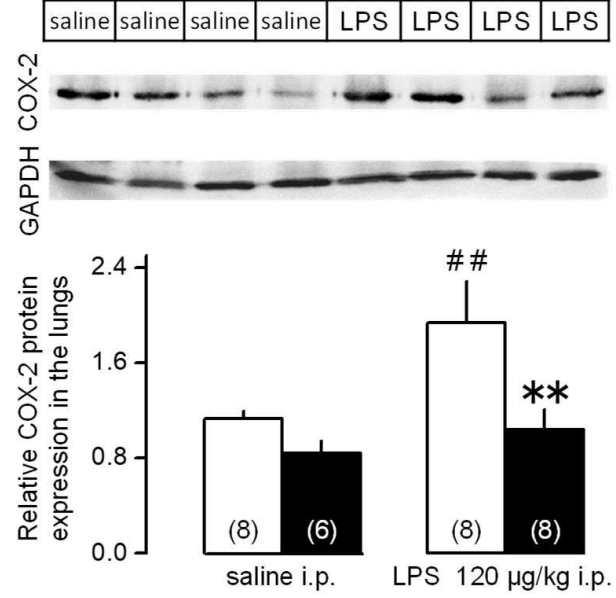

\begin{tabular}{|c|c|c|c|}
\hline $\mathrm{Tacr1}^{+/+}$ & Tacr1 ${ }^{-1-}$ & $\mathrm{TaCr}^{+/ /+}$ & Tacr1 \\
\hline \begin{tabular}{c|c|c|} 
aline & salin
\end{tabular} & \begin{tabular}{l|l} 
aline saline
\end{tabular} & \begin{tabular}{l|l} 
LPS & LP
\end{tabular} & \\
\hline
\end{tabular}
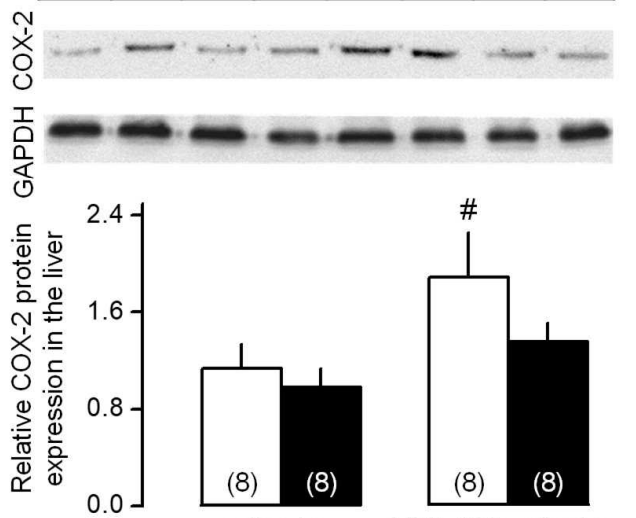

C

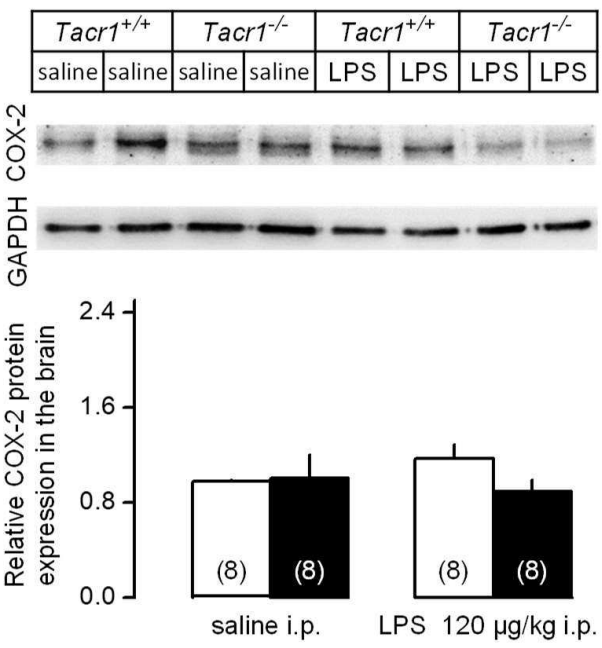

Figure 14. Relative COX-2 protein expression in the lungs (A), liver (B), and in the brain $(C)$ of the Tacr $1^{+/+}$and Tacr $1^{-/}$mice after infusion of LPS or saline. Tissue samples were collected at 40 min postinfusion. Number of animals in the corresponding groups are indicated in the figure. ${ }^{*} P<0.01$, intergenotype difference in the response to LPS; ${ }^{\#} P<0.05$ or ${ }^{\#} P<0.01, L P S$ vs. saline difference within the same genotype. From Pakai et al. [67]. 


\section{Discussion}

We identified novel mechanisms which contribute to the mediation of systemic inflammatory processes. In particular, we showed that 1) hyperbilirubinemia exaggerates the hypothermic response to bacterial endotoxin; 2) genetic ablation of the TRPV1 channel results in minor changes of thermoregulatory phenotype of mice as they use different pattern of thermoeffectors to regulate $\mathrm{T}_{\mathrm{b}} ; 3$ ) in systemic inflammation, TRPV1 has an anti-inflammatory role in the young, which is reversed to pro-inflammatory in older age; 4) the thermoregulatory effects of PACAP38 make the peptide a potential contributor to the febrile response in systemic inflammation; and 5) SP signaling modulates LPS fever through peripheral COX-2 protein expression.

The first novel and unexpected finding was that hyperbilirubinemia in Gunn rats was associated with an exaggerated hypothermic response to high dose of LPS (Fig. 2C) [66]. This exaggeration is unlikely to be due to an attenuation of the ROS-mediated inflammatory signaling by bilirubin. Indeed, attenuating inflammatory signaling should decrease the hypothermic response. Furthermore, while bilirubin, a potent ROS scavenger, has been shown repeatedly to attenuate inflammatory signaling [87-89] we did not find any literature data suggesting that it may amplify inflammatory signaling. An alternative, more reasonable mechanism of the exaggeration of LPS hypothermia might be an action of bilirubin on thermoeffectors. Based on literature findings, Gunn rats respond to administration of exogenous bilirubin (that further intensifies their hyperbilirubinemia) with a pronounced $\left(\sim 3^{\circ} \mathrm{C}\right)$ drop in $T_{b}$ [90]. This drop is likely to reflect decreased thermogenesis, because bilirubin has been demonstrated to depress mitochondrial respiration in liver, heart, and brain tissues in vitro [9193]. In addition, although BAT, which is the main source of nonshivering, temperature-driven thermogenesis in rodents [94] has not been studied, bilirubin is likely to have the same effect in brown fat as in all other tissues studied. A profound decrease in the threshold $\mathrm{T}_{\mathrm{b}}$ for activation of thermogenesis is the principle autonomic thermoeffector mechanism of LPS-induced hypothermia [95]. Although skin vasodilation is not the main mechanism of LPS-induced hypothermia [95], increased skin vasodilation in $\mathrm{J} / \mathrm{J}$ rats could have contributed to their enhanced hypothermic response to LPS as well. Hyperbilirubinemia has been shown to attenuate the pressor effects of angiotensin II in Gunn rats [96] and to lower angiotensin II- 
dependent hypertension in mice $[97,98]$ the latter effect being attributed to the blockade of superoxide production and possibly to enhanced vasorelaxation [97]. In a study conducted in humans [99] hyperbilirubinemia exaggerated endothelium-dependent vasodilation of the brachial artery, which supplies subcutaneous and cutaneous tissues of the arm.

The same action on thermoeffectors could be responsible for the attenuation of the late fever response to the high dose of LPS in $\mathrm{J} / \mathrm{J}$ rats, which was also observed in our study (Fig. 2C) [66]. Since the thermoregulatory response to the high dose is a combination of early hypothermia and late fever, in our case the exaggeration of the hypothermic response may be difficult to distinguish from an independent attenuation of the fever response. Interestingly, the fever response to the low dose of LPS was not affected by hyperbilirubinemia in Gunn rats (Fig. 2B), which was another novel observation. It suggests that ROS signaling is not involved in the febrile response, at least not to weak stimuli.

Compared to $\mathrm{J} /+$ rats, $\mathrm{J} / \mathrm{J}$ rats responded to the high dose of LPS with a blunted surge in GGT (Fig. 4C) [66]. The LPS-induced surges in ALT and AST tended to be reduced, but the magnitude of the reduction did not reach the level of statistical significance (Fig. 4A, B). The revealed high sensitivity of GGT to hyperbilirubinemia was expected. Indeed, GGT is also a marker of oxidative stress, [100] and the blood concentration of this enzyme is inversely related to the blood antioxidant activity $[101,102]$. The observed effect of hyperbilirubinemia on the LPS-induced GGT response and the tendencies revealed (with regard to ALT and AST responses) agree with the previously reported protective action of bilirubin in endotoxin shock [103] and other pathological conditions [104, 105].

As discussed in Pakai et al. [66], the cytoprotective action of bilirubin is likely to be due to the suppression of oxidative stress, either directly (by scavenging ROS) or indirectly (by inhibiting the expression of free radical-generating enzymes, such as NO synthase and nicotinamide adenine dinucleotide phosphate oxidase) [103]. By either mechanism, bilirubin potently suppresses the accumulation of lipophilic ROS in cell membranes $[42,43]$ and the decreased accumulation of ROS prevents cell apoptosis and tissue injury associated with oxidative stress [105]. Yet another mechanism of tissue protection by bilirubin in LPS-induced systemic inflammation could be the exaggeration of hypothermia. Hypothermia is an adaptive, active thermoregulatory response to high doses of LPS and other strong inflammatory stimuli [17]. It involves cold-seeking behavior $[95,106,107]$ and is thought to be aimed at decreasing 
the metabolic requirements of tissues $[16,17]$. In previous studies $[108,109]$, when rats were either allowed to select their preferred low $T_{a}$ or maintained at a lower $T_{a}$ chosen by the investigators during endotoxin shock or Escherichia coli infection, their survival rates increased. At least in some cases, the increased survival was coupled with a suppressed surge in ALT [108].

While markers of hepatocyte damage were affected by hyperbilirubinemia, markers of renal dysfunction were not (Fig. 3), thus suggesting the lack of tissue protection in the kidneys. The observed tissue specificity for the protective action of bilirubin may relate to the fact that the liver is the principal site for bilirubin metabolism. Due to this, bilirubin concentration in hepatocytes should be higher than anywhere else in the body, including renal epitheliocytes. Although bilirubin conjugation is defective in $\mathrm{J} / \mathrm{J}$ rats [110], and these animals excrete only traces of bilirubin in the bile [111], they still have more than a 2-fold-higher concentration of bilirubin in the liver than in the kidneys [112].

After we showed that bilirubin, a potent ROS scavenger, can influence the systemic inflammation response, we studied receptorial mechanisms, which can be also important in addition to ROS. Since the TRPV1 channel and the nerve endings expressing the channel (i.e., capsaicin-sensitive sensory fibers) also play a role in inflammatory processes, also including systemic, next we focused our attention on the role of the TRPV1 channel and on mediators released from TRPV1-expressing neurons in thermoregulation (see Fig. 1). The second novel finding was that the main thermoregulatory abnormality of $\operatorname{Trp} v 1^{-/-}$mice appears to be not an altered level of $T_{b}$, but a different pattern of thermoeffectors used to regulate $T_{b}$ under various thermal, housing, and restraint conditions [65]. Trpv1 KO mice were consistently hypometabolic (had a lower $\mathrm{VO}_{2}$ ) (Fig. 6) and preferred a lower $\mathrm{T}_{\mathrm{a}}$ than controls. These thermoeffector changes tend to decrease $\mathrm{T}_{\mathrm{b}}$. At the same time, the $\mathrm{KO}$ mice expressed two thermoeffector activities aimed at increasing $\mathrm{T}_{\mathrm{b}}$. First, they had more pronounced tail skin vasoconstriction (Fig. 6) and, in agreement with this, had a higher TNZ. Second, they expressed strong hyperkinesis with the average locomotion velocity during the second half of the inactive phase exceeding that of wild-type mice during the active phase. As we discussed in details elsewhere [65], the increased spontaneous locomotion was unlikely to be due to changes in anxiety or exploratory behavior, because the mice were extensively adapted to experimental conditions and observed over long periods of time. Autonomic thermoregulatory responses of 
$\operatorname{Trp} v 1^{-/}$mice showed no attenuation in our study and in studies by others [113-115], while $\operatorname{Trp} v 1^{-/-}$mice preferred a lower $\mathrm{T}_{\mathrm{a}}$ and were hyperactive as compared to the controls. Similar to how humans prefer to exercise in a cooler environment, experimental rodents select a lower $\mathrm{T}_{\mathrm{a}}$ when their activity is higher. A similar adjustment accompanies the exploratory behavior of rats in a thermogradient apparatus when the animals are not adapted to the apparatus [107]. Having excluded profound deficiencies in the two major autonomic effectors directly and knowing that a decrease in the thermopreferendum often occurs secondarily to an increase in locomotor activity, we hypothesized that the increased locomotion may be one of the "primary" symptoms of the Trpv1/- phenotype [65].

After clarifying the role of the TRPV1 channel in the thermoregulation system of mice, we looked at the function of the channel in systemic inflammation. We showed that pharmacological blockade of TRPV1 channels increased LPS-induced mortality in the young (Fig. 7A) [68], and discussed that similar observations have been made in adolescent (6-8 wk) mice and in rats treated with capsazepine $[49,116]$. However, capsazepine is not a highly selective TRPV1 antagonist and has a low potency of blocking the proton mode of TRPV1 activation in the rat and mouse [47]. In fact, a non-TRPV1-mediated effect of capsazepine on the outcome of systemic inflammation has been proposed recently [49]. Our results also agreed with the exaggerated symptoms of LPS-induced shock found in young mature (13-20 wk) Trpv1/- mice [48]. The effect of AMG517 on LPS-induced systemic inflammation in aged mice was the opposite to that found in young mice, the effect on $T_{b}$ was qualitatively the same. It is possible that the role of TRPV1 in different functions changes with age in a different way. In the regulation of locomotor activity [65, 117] and inflammation [68], the role of TRPV1 reverses with age. In the modulation of $\mathrm{T}_{\mathrm{b}}[118]$, it does not. In the regulation of body mass, TRPV1 channels are either uninvolved [65] or counteract obesity [114] in the young but promote obesity in the aged $[65,117]$.

As reviewed by others [49, 119] many treatments affect mortality in LPS-induced aseptic inflammation and CLP-induced sepsis in opposite ways, confirming that the systemic inflammatory response per se can be harmful to the host, even though it is crucial for defending the host against infection $[35,36]$. For example, mice with a dysfunctional toll-like receptor 4 are resistant to LPS but are highly susceptible to Gram-negative bacterial infection [120-122]. This susceptibility to infection can be reserved by pretreating the toll-like receptor 4-deficient 
mice with TNF and interleukin-1 $\alpha$ [121]. In contrast to aseptic SIRS, polymicrobial sepsis (induced by CLP) caused accelerated mortality in aged $\operatorname{Tr} p v 1^{-/-}$mice as compared with wildtype littermates. The recovery of $\operatorname{Trpv1^{-/}}$ mice from hypothermia associated with the CLP procedure was delayed [68]. Overall, prior literature data obtained in young rodents (adolescents to mature adults) and the experiment with AMG517 in young adult mice show that the effects of TRPV1 blockade on both LPS-induced SIRS and antibiotic-treated sepsis vary from none to strong exaggeration of severity and mortality $[48,49,116]$, whereas the effect of TRPV1 blockade on mortality in untreated sepsis is the opposite: attenuation [123].

Mechanisms of the age-associated reversal of the anti-inflammatory role of TRPV1 are unknown. Our TNF- $\alpha$ data suggest that the reversal occurs at initial stages of the pathogenesis of SIRS, at or upstream of TNF- $\alpha$ production [68]. The TNF- $\alpha$ response to LPS has been shown to be under suppressive control of TRPV1 channels on sensory nerves [124]. Loss of TRPV1mediated suppression of TNF- $\alpha$ production in aged animals may reflect reduced translation of the TRPV1 protein and its reduced transport to the periphery [125], possibly due to ageassociated decline in neurotrophic support to ganglionic neurons [126]. Changes in TNF- $\alpha$ production may be central to aging-related changes in the pathobiology of sepsis: elderly patients respond to infection, including septic shock, with higher TNF- $\alpha[127,128]$, and inflammatory cytokine production in intensive-care-unit patients with sepsis is affected by TNF- $\alpha$-related genetic polymorphisms [129].

Since the activation of TRPV1 channels on neural endings (i.e., on capsaicin-sensitive afferent nerve fibers) results in the release of different inflammatory substances, also including SP and PACAP38, in the remaining of the present work, we also wanted to study the thermoregulatory effects of these substances. Our findings on the thermoregulatory response to PACAP clearly demonstrate that central, rather than peripheral mechanisms are involved in the hyperthermia-inducing effect of PACAP38 [64]. Although PACAP38-induced hyperthermia has been studied earlier in small rodents, based on data available from those experiments, no firm conclusion could be drawn about the site of the thermoregulatory action of PACAP38. To fill this gap, we compared the effects of central and systemic administration, and showed that the same or even a tenfold lower dose of PACAP3 8 evokes stronger hyperthermia, when given i.c.v. than when delivered i.v. This result unequivocally supports the central mediation hypothesis. The slight increase of $T_{c}$ in response to peripherally infused PACAP38 could be 
attributed to its penetration of the blood-brain barrier [130, 131]. To our knowledge, ours was the first report [64], in which the central and peripheral thermoregulatory responses to PACAP38 were compared under identical experimental conditions.

We also studied the characteristics of the thermoregulatory response to PACAP38 and found that the hyperthermia started to develop promptly: already 10 min after drug administration, we could detect the activation of thermoeffectors and a slight increase of $T_{c}$ (Fig. 9). This was a novel finding of the study as in all of the earlier studies investigating the thermal effect of PACAP38, $\mathrm{T}_{\mathrm{b}}$ was recorded hourly and the substance was administered in a stressful manner, thus the developing stress-induced hyperthermia, which was also present in the vehicle-treated animals masked the early phase of the response. We found that PACAP38 administration resulted in the simultaneous activation of non-shivering thermogenesis and cutaneous vasoconstriction (Fig. 9), which are the two principal autonomic cold-defense thermoeffectors [132]. The initial skin vasoconstriction seems to contradict the reported vasodilatory effect of PACAP38 [77, 78], but this contradiction can be resolved by considering that in the current study PACAP38 was delivered into the lateral ventricle of the brain, from where it can broadly access the POA, where neurons of the thermoeffector pathway for tail skin vasomotor tone are situated $[83,133]$. Therefore, it is plausible that PACAP38 acted on central thermoregulatory elements resulting in skin vasoconstriction, but when the peptide spread to more distant (non-thermoregulatory) areas, it caused vasodilatory effect, which was also observed in our study 40 min after PACAP38 injection (Fig. 9) [64].

As an alternative approach to study the role of PACAP in thermoregulation, we investigated deep $\mathrm{T}_{\mathrm{b}}$ and locomotor activity of $\mathrm{Pacap}^{-/-}$mice and found that these mice were hyperactive throughout the day and hyperthermic during the light phase as compared to controls (Fig. 10A). The hyperactivity of Pacap ${ }^{-/}$mice was also observed in an earlier study [134], although another group reported no alteration in the locomotor activity of the Pacap ${ }^{-/ 2}$ mice compared to controls [135]. It was a novel finding in our experiments [64], that the increased activity of the Pacap $^{-/-}$mice resulted in elevated $\mathrm{T}_{\mathrm{b}}$ during the light phase of the day, in which phase locomotor activity correlates strongly with $T_{b}[81,136]$. In contrast to our findings, in the study by Hashimoto et al. [137] Pacap $^{-/-}$mice had lower $\mathrm{T}_{\mathrm{b}}$ during the night than controls, but those experiments were conducted at a $\mathrm{T}_{\mathrm{a}}$ of $23^{\circ} \mathrm{C}$, which could be presumably below the TNZ of mice. Locomotor activity is widely viewed as a thermoregulatory effector in mice $[65$, 
138, 139]. Our findings suggest that freely moving Pacap $^{-/-}$mice utilize locomotor activity as a thermoeffector to maintain an elevated $\mathrm{T}_{b}$ during the light phase and normal $\mathrm{T}_{\mathrm{b}}$ during the night phase of the day.

We then asked whether the basal daytime $\mathrm{T}_{\mathrm{b}}$ of loosely restrained Pacap ${ }^{-/-}$mice (i.e., those that can not use locomotion as a thermoeffector) also differs from their wild-type littermates. In contrast to our results in freely moving animals, when restrained, Pacap ${ }^{-/-}$mice were hypometabolic and had lower $\mathrm{T}_{\mathrm{b}}$ than controls (Fig 10B) [64]. The decreased metabolic rate and $\mathrm{T}_{\mathrm{b}}$ in the absence of PACAP is in harmony with our results demonstrating the hypermetabolic and hyperthermic effect of PACAP38 injection in rats. When we measured the expression of c-Fos positive cells in the POA of the mice, we found that the number of c-Fos positive cells in the MPO was markedly higher in Pacap $^{-/}$mice than in controls, suggesting that the absence of PACAP results in an increased activation of MPO neurons. Since GABAergic neurons in the MPO tonically suppress thermogenesis [84], we proposed that in Pacap $^{-/-}$mice inhibitory MPO neurons are more activated and this results in an enhanced suppression of thermogenesis [64]. This hypothesis is also in harmony with an action of PACAP38 injection on GABAergic MnPO neurons, because activation of these neurons results in an increased suppression of the inhibitory MPO neurons, which leads to elevated metabolic rate and hyperthermia. Although alternate explanations are also plausible, it can be assumed that the absence of PACAP38 results in a lower resting metabolic rate (and $\mathrm{T}_{\mathrm{b}}$ ) and as a compensatory mechanism for the hypometabolism, Pacap $^{-/}$mice become hyperkinetic to maintain normal (or even higher) $\mathrm{T}_{\mathrm{b}}$. Interestingly, a similarly altered thermoeffector pattern (hypometabolism and hyperkinesis) was observed with the abovementioned mice lacking the TRPV1 channel (see above). The similar thermoregulatory consequences of the absence of PACAP and TRPV1 can be explained with the alteration of the same neural pathways as PACAP38 is released from activated capsaicin-sensitive (i.e., TRPV1-expressing) neural afferents into the systemic circulation [51].

Last, we also studied the role of SP signaling in thermoregulation [67]. We showed that the absence of the NK1 receptor results in the attenuation of LPS-induced fever for the first time by using Tacr $^{-/-}$mice (Fig. 12A, B). Our experimental model allowed us to detect the suppression of the febrile response already in the early phase of fever (starting from $\sim 40$ min post-LPS infusion), which was also a novel finding. When we looked at the molecular 
mechanism, we did not find a difference in the $\mathrm{PGE}_{2}$-induced febrile response between $\mathrm{Tacr}^{+/+}$ and $\mathrm{TaCr}^{-/-}$mice. The LPS-induced serum cytokine production and COX-2 mRNA expression in the lungs, liver, and brain of the mice were also statistically indistinguishable between the genotypes. In contrast with mRNA, when we measured COX-2 expression at the protein level, we found that the LPS-induced surge was significantly attenuated in the lungs and tended to be suppressed in the liver of Tacr $1^{-/-}$mice as compared with their Tacr $^{+/+}$littermates.

As discussed in details in Pakai et al. [67], the involvement of SP signaling and the NK1 receptor in experimental fever was reported in earlier studies [59-62, 140]. Antagonists of SP reduced the febrile response to LPS in rats and guinea pigs from the beginning of the response, which was detectable 45-90 min after LPS infusion [59, 60]. However, when the authors looked at the mechanisms connecting the NK1 receptor with the fever signaling pathway, they focused on the later phases of fever (i.e., $2 \mathrm{~h}$ or more post-LPS infusion), presumably, because the early phase was absent in their experiments due to stress-hyperthermia as a consequence of stressful (needle-pinch) drug injection [62]. We conducted the experiments under such conditions (extensive habituation, moderate LPS dose, non-stressful substance administration, and near neutral ambient temperature) that allowed us to study LPS-induced fever from 40 to $360 \mathrm{~min}$ postinfusion in mice, thus we could detect the attenuation of the response already at $40 \mathrm{~min}$ in the absence of Tacrl gene.

The later phases of fever are mediated mostly by increased $\mathrm{PGE}_{2}$ production in the brain, and it is well established that brain-derived $\mathrm{PGE}_{2}$ is an important mediator for the maintenance of LPS-induced fever [141-144]. However, the early phase of fever is triggered from peripheral organs such as the lungs and the liver $[85,86]$. Therefore, our results suggest that the genetic blockade of the NK1 receptor interferes with fever signaling at a peripheral site of action in the early phase of LPS-induced fever. We further supported the peripheral action site of NK1 receptor in fever by showing that the $\operatorname{Tacr}^{-/-}$mice were equally able to increase their thermogenesis and deep $\mathrm{T}_{\mathrm{b}}$ in response to i.c.v. administration of $\mathrm{PGE}_{2}$, then we focused on exploring which step of fever signaling is altered in the Tacr $1^{-/}$mice [67]. In the periphery, SP signaling has been shown to play a role in the induction of pyrogenic cytokine production by macrophages [145] and in pulmonary macrophage activation [146]. In acute lung injury after burns, SP was found to upregulate COX-2 activity [147]. The expression of the NK1 receptor by macrophages is well documented [148-153], but it was also found in various other immune 
cells [154]. We did not find difference in the serum levels of inflammatory cytokines (TNF- $\alpha$ and IL-6) between the LPS-treated Tacrl ${ }^{+/+}$and $\mathrm{Tacrl}^{-/-}$mice, which indicates that the activation of macrophages and their cytokine production is not impaired in the absence of the NK1 receptor. We did not measure the levels of the third major pro-inflammatory cytokine, IL$1 \beta$, because it has been shown that it exerts its pyrogenic actions independently from the NK1 receptor [62]. LPS can also modulate COX-2 transcriptionally and posttranscriptionally in macrophages independently from inflammatory cytokines [155]. When we determined the COX-2 mRNA expression, we found that at this early time point ( $\sim 40 \mathrm{~min})$ it was greatly amplified in the lungs and liver, and to lesser extent in the brain of the mice [67], which results are in harmony with the previous findings [85]. The lack of difference between the genotypes indicates that transcriptional upregulation of COX-2 is not influenced by the NK1 receptor. The correlation of mRNA and protein levels in biological samples is often poor [156], moreover, the expression of COX-2 is regulated not only at the level of transcription but also at the levels of post-transcription and translation [155, 157, 158]. Therefore, we also determined the expression of the COX-2 protein in the lungs, liver, and brain of the mice [67]. In accordance with previous reports showing augmented expression of the COX-2 protein in the periphery [86], we also detected LPS-induced amplification of the COX-2 protein expression in the lungs and liver of Tacrl $^{+/+}$mice as compared with saline treatment, whereas we did not find significant increase in their brain. Importantly, however, the LPS-induced amplification of the expression of the COX-2 protein was attenuated in the lungs and tended to be suppressed in the liver of $\mathrm{Tacrl}^{-/-}$mice as compared with their $\mathrm{Tacrl}^{+/+}$littermates. In accordance with the different COX-2 protein expression between the genotypes, the administration of LPS caused a significant surge of $\mathrm{PGE}_{2}$ concentration in the lungs of $T a c r 1^{+/+}$mice, which was absent in the Tacrl $^{-/-}$mice. The sensitive site where PGEs produce fever is located within the region of the brain that includes the organum vasculosum laminae terminalis and the surrounding POA of the hypothalamus [159]. Peripherally borne $\mathrm{PGE}_{2}$ can broadly penetrate in the perivascular space in the periventricular organs (such as the organum vasculosum laminae terminalis) and activate neurons or non-neural cells, thus trigger the febrile response [160]. It has to be noted that fever signaling was not examined at later time points in the current experiments due to the study design, and therefore it cannot be excluded that COX-2 expression and $\mathrm{PGE}_{2}$ production in the brain are also affected by the blockade of the NK1 receptor, especially during the maintenance phase of fever. In sum, our results demonstrated that at the onset of the fever 
response the NK1 receptor contributes to the augmentation of COX-2 protein expression in peripheral organs, for further discussion, see Pakai et al. [67]. The exact mechanism, how SP signaling interacts with COX-2 expression remains to be elucidated in future studies.

Taken together, these findings can help the better understanding of the pathophysiological mechanisms involved in inflammatory processes and, as a perspective, can serve as the bases of new prognostic and therapeutical approaches in patients with systemic inflammation, including sepsis. We explored ligand, receptor, and neurotransmitter mechanisms which play a part in the processes of systemic inflammation. By further extending the plethora of inflammatory mediators, these results can advance our knowledge about the pathophysiology of systemic inflammation, and, from a translational research point of view, they can open new ways for the development of diagnostic and prognostic tools as well as for prevention and perhaps for drug development. 


\section{Summary and conclusions}

1) We showed that hyperbilirubinemia in $\mathrm{J} / \mathrm{J}$ Gunn rats was associated with a marked exaggeration of the early hypothermic response to the high dose of LPS, supposedly through a direct inhibition of nonshivering thermogenesis by bilirubin and possibly also through a direct vasodilatatory action of bilirubin in the skin. This novel, hypothermia-exaggerating effect might be responsible, at least in part, for the observed tendency of $\mathrm{J} / \mathrm{J}$ rats to respond to the high dose of LPS with attenuated hepatic damage. Hyperbilirubinemia in Gunn rats was also associated with a deep attenuation of the late febrile response to the high dose of LPS, but did not attenuate

the fever response to the low dose. The attenuation of the fever response to high dose of LPS could be due to either direct actions of bilirubin on thermoeffectors (inhibition of nonshivering thermogenesis and induction of skin vasodilation) or the ROS-scavenging action of bilirubin attenuating pyrogenic signaling. However, the experiments with the low dose strongly suggest that ROS signaling is not involved in the fever response to low doses of LPS [66].

2) We described that $\operatorname{Trpv} 1 \mathrm{KO}$ mice possess a distinct thermoregulatory phenotype, which includes hypometabolism, enhanced skin vasoconstriction, preference for a lower $\mathrm{T}_{\mathrm{a}}$, and an increased locomotor activity [65].

3) We showed that the anti-inflammatory role firmly established for TRPV1 channels in young rodents $[48,49,116]$ is reversed with aging [68]. Whereas pharmacological or genetic TRPV1 antagonism decreases the survival rate in aseptic SIRS and in antibiotic-treated sepsis in the young, both types of TRPV1 antagonism have the opposite effect on aseptic SIRS in middle-aged mice. The age-dependent reversal of the anti-inflammatory role of TRPV1 to proinflammatory is likely due, at least in part, to a reversal of the suppressive control of TRPV1 on TNF- $\alpha$ production. These pathobiological changes are highly important, as evident from the decreased ability of aged $\operatorname{Trp} v 1^{-/-}$mice to resist polymicrobial sepsis. These findings may influence the development of TRPV1 antagonists, widely viewed as new-generation painkillers $[47,73,161-163]$. If what we found for murine models applies to human sepsis, anti-TRPV1 therapy may suppress the systemic inflammatory response in 
the previously uninfected (untreated with antibiotics) elderly and, hence, decrease their resistance to bacterial infection and sepsis. This potential side effect is especially serious, because recognition of infection is often complicated in older patients by a variety of factors, including the absence of fever, which often delays treatment $[164,165]$.

4) We showed that PACAP38 causes hyperthermia by acting on targets within the CNS. The PACAP38-induced hyperthermia is brought about through the simultaneous activation of both autonomic cold-defense effectors: elevation of nonshivering thermogenesis and cutaneous vasoconstriction. We hypothesize that GABAergic neurons within the MnPO are involved in mediation of thermoregulatory response to PACAP38. The absence of PACAP results in hyperkinesis and daytime hyperthermia in freely-moving Pacap ${ }^{-/}$mice through mechanisms which need to be clarified, but an involvement of TRPV1 and altered central biochemical processes can be suspected. The increased locomotor activity is presumably a compensatory mechanism for the hypometabolism and hypothermia, which is present under resting conditions in the absence of PACAP [64].

5) We showed that the NK1 receptor contributes to the early phase of LPS-induced fever by enhancing COX-2 protein expression in the periphery. These findings about the role of the NK1 receptor in LPS-induced fever further advance our understanding about the interactions between SP signaling and the "cytokine-COX2-PGE 2 " axis in experimental fever [67]. As a perspective, these results can help to identify NK1 receptor as a drug target, as recently proposed against the novel coronavirus (COVID-19) [166]. 


\section{Acknowledgement}

I would like to express my thanks to my supervisor, Dr. András Garami for tutoring me through all these years, and for his help and advice in my experimental work.

I am most grateful to Professors Miklós Székely and Zoltán Szelényi, who gave me the opportunity to start working at their Department years ago, and I appreciate their scientific advice and knowledge, and continuous support and encouragement in my research. I am also very grateful to Prof. Ákos Koller for his many helpful discussions and suggestions.

I especially thank Prof. Andrej A. Romanovsky for offering me the opportunity to work in FeverLab at St. Joseph's Hospital and Medical Center (Phoenix, Arizona, USA), for always providing guidance, assistance, help and support when I needed. I thank Andrej for encouraging my research and for allowing me to grow as a research scientist. I am also very grateful to Dr. Samuel Wanner from FeverLab for his explanations, stimulating discussions and for solving both technical and non-technical issues during the cooperative work.

I would like to extend my gratitude to Prof. Péter Hegyi, the Director of the Translational Medicine Program and Head of the Institute for providing the inspiring environment in which we conducted the experiments and for the opportunity to work and study in his Program at the Doctoral School of Clinical Medicine in Szeged.

I would like to also thank the members of our research group for helping me through all these years regarding both the theoretical and technical parts of the research. I also thank Anikó Várnagyné Rózsafi for her valuable technical assistance in my experiments.

I would like to thank Prof. Dóra Reglödi at the Department of Anatomy, Medical School, University of Pécs for her cooperative work and for giving me the opportunity to join the PACAP research projects. Many thanks to Drs. Andrea Tamás, Balázs Gaszner, and Eszter Bánki for their expert help with the PACAP projects, particularly for their great contribution to the immunohistochemistry experiments.

I would like to thank Prof. Erika Pintér at the Department of Pharmacology and Pharmacotherapy, Medical School, University of Pécs for her help in designing the NK1 receptor project and for solving both technical and non-technical issues during the cooperative 
work. I am also grateful to Drs. Valéria Tékus, Ágnes Kemény, Krisztina Pohóczky for their expert help in conducting the tissue harvesting and the majority of molecular biology experiments (ELISA, PCR) in the project.

I would like to thank Katalin Ördög and Drs. László Deres and Róbert Mátics for their expert help with regard to the Western blotting.

Last but not least, I wish to express my warmest thanks to my family for their infinite support and patience during my PhD studies.

The author's research summarized in this work has been supported in part by the Hungarian National Research, Development and Innovation Office (grant FK 124483), GINOP "Stay alive - translational medicine for better healthcare" (2.3.2-15-2016-00048), EFOP "Live longer" (3.6.2-16-2017-00006), and the János Bolyai Research Scholarship of the Hungarian Academy of Sciences. 


\section{References}

[1] Garami A, Steiner AA, Romanovsky AA. Fever and hypothermia in systemic inflammation. Handb Clin Neurol 2018;157:565-97.

[2] Balk RA. Systemic inflammatory response syndrome (SIRS): where did it come from and is it still relevant today? Virulence 2014;5:20-6.

[3] Bone RC, Balk RA, Cerra FB, Dellinger RP, Fein AM, Knaus WA, et al. Definitions for sepsis and organ failure and guidelines for the use of innovative therapies in sepsis. The ACCP/SCCM Consensus Conference Committee. American College of Chest Physicians/Society of Critical Care Medicine. Chest 1992;101:1644-55.

[4] Levy MM, Fink MP, Marshall JC, Abraham E, Angus D, Cook D, et al. 2001 SCCM/ESICM/ACCP/ATS/SIS International Sepsis Definitions Conference. Crit Care Med 2003;31:1250-6.

[5] Singer M, Deutschman CS, Seymour CW, Shankar-Hari M, Annane D, Bauer M, et al. The Third International Consensus Definitions for Sepsis and Septic Shock (Sepsis-3). JAMA 2016;315:801-10.

[6] Rudd KE, Johnson SC, Agesa KM, Shackelford KA, Tsoi D, Kievlan DR, et al. Global, regional, and national sepsis incidence and mortality, 1990-2017: analysis for the Global Burden of Disease Study. Lancet 2020;395:200-11.

[7] Sakr Y, Jaschinski U, Wittebole X, Szakmany T, Lipman J, Namendys-Silva SA, et al. Sepsis in Intensive Care Unit Patients: Worldwide Data From the Intensive Care over Nations Audit. Open Forum Infect Dis 2018;5:ofy313.

[8] Quartin AA, Schein RM, Kett DH, Peduzzi PN. Magnitude and duration of the effect of sepsis on survival. Department of Veterans Affairs Systemic Sepsis Cooperative Studies Group. JAMA 1997;277:1058-63.

[9] Dreiher J, Almog Y, Sprung CL, Codish S, Klein M, Einav S, et al. Temporal trends in patient characteristics and survival of intensive care admissions with sepsis: a multicenter analysis*. Crit Care Med 2012;40:855-60.

[10] Walkey AJ, Lagu T, Lindenauer PK. Trends in sepsis and infection sources in the United States. A population-based study. Ann Am Thorac Soc 2015;12:216-20. 
[11] Angus DC, Linde-Zwirble WT, Lidicker J, Clermont G, Carcillo J, Pinsky MR. Epidemiology of severe sepsis in the United States: analysis of incidence, outcome, and associated costs of care. Crit Care Med 2001;29:1303-10.

[12] Rhee C, Dantes R, Epstein L, Murphy DJ, Seymour CW, Iwashyna TJ, et al. Incidence and Trends of Sepsis in US Hospitals Using Clinical vs Claims Data, 2009-2014. JAMA 2017;318:1241-9.

[13] Atkins E. Fever: its history, cause, and function. Yale J Biol Med 1982;55:283-9.

[14] Scott A, Khan KM, Cook JL, Duronio V. What is "inflammation"? Are we ready to move beyond Celsus? Br J Sports Med 2004;38:248-9.

[15] Saper CB, Romanovsky AA, Scammell TE. Neural circuitry engaged by prostaglandins during the sickness syndrome. Nat Neurosci 2012;15:1088-95.

[16] Romanovsky AA, Almeida MC, Aronoff DM, Ivanov AI, Konsman JP, Steiner AA, et al. Fever and hypothermia in systemic inflammation: recent discoveries and revisions. Front Biosci 2005;10:2193-216.

[17] Romanovsky AA, Szekely M. Fever and hypothermia: two adaptive thermoregulatory responses to systemic inflammation. Med Hypotheses 1998;50:219-26.

[18] Corrigan JJ, Fonseca MT, Flatow EA, Lewis K, Steiner AA. Hypometabolism and hypothermia in the rat model of endotoxic shock: independence of circulatory hypoxia. J Physiol 2014;592:3901-16.

[19] Fonseca MT, Rodrigues AC, Cezar LC, Fujita A, Soriano FG, Steiner AA. Spontaneous hypothermia in human sepsis is a transient, self-limiting, and nonterminal response. $\mathrm{J}$ Appl Physiol (1985) 2016;120:1394-401.

[20] Taniguchi T, Kanakura H, Takemoto Y, Yamamoto K. Effects of hypothermia on mortality and inflammatory responses to endotoxin-induced shock in rats. Clin Diagn Lab Immunol 2003;10:940-3.

[21] Villar J, Slutsky AS. Effects of induced hypothermia in patients with septic adult respiratory distress syndrome. Resuscitation 1993;26:183-92.

[22] Arons MM, Wheeler AP, Bernard GR, Christman BW, Russell JA, Schein R, et al. Effects of ibuprofen on the physiology and survival of hypothermic sepsis. Ibuprofen in Sepsis Study Group. Crit Care Med 1999;27:699-707. 
[23] Clemmer TP, Fisher CJ, Jr., Bone RC, Slotman GJ, Metz CA, Thomas FO. Hypothermia in the sepsis syndrome and clinical outcome. The Methylprednisolone Severe Sepsis Study Group. Crit Care Med 1992;20:1395-401.

[24] Kushimoto S, Gando S, Saitoh D, Mayumi T, Ogura H, Fujishima S, et al. The impact of body temperature abnormalities on the disease severity and outcome in patients with severe sepsis: an analysis from a multicenter, prospective survey of severe sepsis. Crit Care 2013;17:R271.

[25] Rumbus Z, Matics R, Hegyi P, Zsiboras C, Szabo I, Illes A, et al. Fever Is Associated with Reduced, Hypothermia with Increased Mortality in Septic Patients: A MetaAnalysis of Clinical Trials. PLoS One 2017;12:e0170152.

[26] Steiner AA, Fonseca MT, Soriano FG. Should we assume that hypothermia is a dysfunction in sepsis? Crit Care 2017;21:8.

[27] Le Gall JR, Lemeshow S, Saulnier F. A new Simplified Acute Physiology Score (SAPS II) based on a European/North American multicenter study. JAMA 1993;270:2957-63.

[28] Knaus WA, Draper EA, Wagner DP, Zimmerman JE. APACHE II: a severity of disease classification system. Crit Care Med 1985;13:818-29.

[29] Opal SM. Concept of PIRO as a new conceptual framework to understand sepsis. Pediatr Crit Care Med 2005;6:S55-60.

[30] Beverly A, Walter E, Carraretto M. Management of hyperthermia and hypothermia in sepsis: A recent survey of current practice across UK intensive care units. J Intensive Care Soc 2016;17:88-9.

[31] Roth J, Blatteis CM. Mechanisms of fever production and lysis: lessons from experimental LPS fever. Compr Physiol 2014;4:1563-604.

[32] Bode JG, Ehlting C, Haussinger D. The macrophage response towards LPS and its control through the p38(MAPK)-STAT3 axis. Cell Signal 2012;24:1185-94.

[33] Tollner B, Roth J, Storr B, Martin D, Voigt K, Zeisberger E. The role of tumor necrosis factor (TNF) in the febrile and metabolic responses of rats to intraperitoneal injection of a high dose of lipopolysaccharide. Pflugers Arch 2000;440:925-32.

[34] Cohen J. The immunopathogenesis of sepsis. Nature 2002;420:885-91.

[35] Rittirsch D, Flierl MA, Ward PA. Harmful molecular mechanisms in sepsis. Nat Rev Immunol 2008;8:776-87. 
[36] Stearns-Kurosawa DJ, Osuchowski MF, Valentine C, Kurosawa S, Remick DG. The pathogenesis of sepsis. Annu Rev Pathol 2011;6:19-48.

[37] Romanovsky AA, Garami A. Prostaglandin riddles in energy metabolism: E is for excess, D is for depletion. Focus on "Food deprivation alters thermoregulatory responses to lipopolysaccharide by enhancing cryogenic inflammatory signaling via prostaglandin D2". Am J Physiol Regul Integr Comp Physiol 2010;298:R1509-11.

[38] Steiner AA, Hunter JC, Phipps SM, Nucci TB, Oliveira DL, Roberts JL, et al. Cyclooxygenase-1 or -2--which one mediates lipopolysaccharide-induced hypothermia? Am J Physiol Regul Integr Comp Physiol 2009;297:R485-94.

[39] Lazarus M, Yoshida K, Coppari R, Bass CE, Mochizuki T, Lowell BB, et al. EP3 prostaglandin receptors in the median preoptic nucleus are critical for fever responses. Nat Neurosci 2007;10:1131-3.

[40] Steiner AA, Branco LG. Fever and anapyrexia in systemic inflammation: intracellular signaling by cyclic nucleotides. Front Biosci 2003;8:s1398-408.

[41] Hampton MB, Kettle AJ, Winterbourn CC. Inside the neutrophil phagosome: oxidants, myeloperoxidase, and bacterial killing. Blood 1998;92:3007-17.

[42] Sedlak TW, Saleh M, Higginson DS, Paul BD, Juluri KR, Snyder SH. Bilirubin and glutathione have complementary antioxidant and cytoprotective roles. Proc Natl Acad Sci U S A 2009;106:5171-6.

[43] Stocker R, Yamamoto Y, McDonagh AF, Glazer AN, Ames BN. Bilirubin is an antioxidant of possible physiological importance. Science 1987;235:1043-6.

[44] Botz B. The role of sensory neuropeptides in mouse models of neuropathy and immune arthritis (Doctoral Thesis). Department of Pharmacology and Pharmacotherapy, Medical School, University of Pecs, Pecs, Hungary 2015.

[45] Dux M, Rosta J, Pinter S, Santha P, Jancso G. Loss of capsaicin-induced meningeal neurogenic sensory vasodilatation in diabetic rats. Neuroscience 2007;150:194-201.

[46] Dux M, Rosta J, Santha P, Jancso G. Involvement of capsaicin-sensitive afferent nerves in the proteinase-activated receptor 2-mediated vasodilatation in the rat dura mater. Neuroscience 2009; 161:887-94.

[47] Romanovsky AA, Almeida MC, Garami A, Steiner AA, Norman MH, Morrison SF, et al. The transient receptor potential vanilloid-1 channel in thermoregulation: a thermosensor it is not. Pharmacol Rev 2009;61:228-61. 
[48] Clark N, Keeble J, Fernandes ES, Starr A, Liang L, Sugden D, et al. The transient receptor potential vanilloid 1 (TRPV1) receptor protects against the onset of sepsis after endotoxin. FASEB J 2007;21:3747-55.

[49] Guptill V, Cui X, Khaibullina A, Keller JM, Spornick N, Mannes A, et al. Disruption of the transient receptor potential vannilloid 1 can affect survival, bacterial clearance, and cytokine gene expression during murine sepsis. Anesthesiology 2011;114:1190-9.

[50] Helyes Z, Kun J, Dobrosi N, Sandor K, Nemeth J, Perkecz A, et al. Pituitary Adenylate Cyclase-Activating Polypeptide Is Upregulated in Murine Skin Inflammation and Mediates Transient Receptor Potential Vanilloid-1-Induced Neurogenic Edema. J Invest Dermatol 2015;135:2209-18.

[51] Helyes Z, Pozsgai G, Borzsei R, Nemeth J, Bagoly T, Mark L, et al. Inhibitory effect of PACAP-38 on acute neurogenic and non-neurogenic inflammatory processes in the rat. Peptides 2007;28:1847-55.

[52] Kemeny A, Reglodi D, Cseharovszky R, Hashimoto H, Baba A, Szolcsanyi J, et al. Pituitary adenylate cyclase-activating polypeptide deficiency enhances oxazoloneinduced allergic contact dermatitis in mice. J Mol Neurosci 2010;42:443-9.

[53] Das M, Vihlen CS, Legradi G. Hypothalamic and brainstem sources of pituitary adenylate cyclase-activating polypeptide nerve fibers innervating the hypothalamic paraventricular nucleus in the rat. J Comp Neurol 2007;500:761-76.

[54] Joo KM, Chung YH, Kim MK, Nam RH, Lee BL, Lee KH, et al. Distribution of vasoactive intestinal peptide and pituitary adenylate cyclase-activating polypeptide receptors (VPAC1, VPAC2, and PAC1 receptor) in the rat brain. J Comp Neurol 2004;476:388-413.

[55] Machado NLS, Bandaru SS, Abbott SBG, Saper CB. EP3R-Expressing Glutamatergic Preoptic Neurons Mediate Inflammatory Fever. J Neurosci 2020;40:2573-88.

[56] Palkovits M, Somogyvari-Vigh A, Arimura A. Concentrations of pituitary adenylate cyclase activating polypeptide (PACAP) in human brain nuclei. Brain Res 1995;699:116-20.

[57] Resch JM, Boisvert JP, Hourigan AE, Mueller CR, Yi SS, Choi S. Stimulation of the hypothalamic ventromedial nuclei by pituitary adenylate cyclase-activating polypeptide induces hypophagia and thermogenesis. Am J Physiol Regul Integr Comp Physiol 2011;301:R1625-34. 
[58] Resch JM, Maunze B, Gerhardt AK, Magnuson SK, Phillips KA, Choi S. Intrahypothalamic pituitary adenylate cyclase-activating polypeptide regulates energy balance via site-specific actions on feeding and metabolism. Am J Physiol Endocrinol Metab 2013;305:E1452-63.

[59] Blatteis CM, Xin L, Quan N. Neuromodulation of fever. A possible role for substance P. Ann N Y Acad Sci 1994;741:162-73.

[60] Szelenyi Z, Szekely M, Balasko M. Role of substance P (SP) in the mediation of endotoxin (LPS) fever in rats. Ann N Y Acad Sci 1997;813:316-23.

[61] Balasko M, Szekely M, Szelenyi Z. The effect of CP-96,345, a non-peptide substance$\mathrm{P}$ antagonist, on thermoregulation and the development of endotoxin-fever in rats. $\mathrm{J}$ Therm Biol 2000;25:1-4.

[62] Reis RC, Brito HO, Fraga D, Cabrini DA, Zampronio AR. Central substance P NK(1) receptors are involved in fever induced by LPS but not by IL-1beta and CCL3/MIP1alpha in rats. Brain Res 2011;1384:161-9.

[63] Hubbard WJ, Choudhry M, Schwacha MG, Kerby JD, Rue LW, 3rd, Bland KI, et al. Cecal ligation and puncture. Shock 2005;24 Suppl 1:52-7.

[64] Banki E, Pakai E, Gaszner B, Zsiboras C, Czett A, Bhuddi PR, et al. Characterization of the thermoregulatory response to pituitary adenylate cyclase-activating polypeptide in rodents. J Mol Neurosci 2014;54:543-54.

[65] Garami A, Pakai E, Oliveira DL, Steiner AA, Wanner SP, Almeida MC, et al. Thermoregulatory phenotype of the Trpv1 knockout mouse: thermoeffector dysbalance with hyperkinesis. J Neurosci 2011;31:1721-33.

[66] Pakai E, Garami A, Nucci TB, Ivanov AI, Romanovsky AA. Hyperbilirubinemia exaggerates endotoxin-induced hypothermia. Cell Cycle 2015;14:1260-7.

[67] Pakai E, Tekus V, Zsiboras C, Rumbus Z, Olah E, Keringer P, et al. The Neurokinin-1 Receptor Contributes to the Early Phase of Lipopolysaccharide-Induced Fever via Stimulation of Peripheral Cyclooxygenase-2 Protein Expression in Mice. Front Immunol 2018;9:166.

[68] Wanner SP, Garami A, Pakai E, Oliveira DL, Gavva NR, Coimbra CC, et al. Aging reverses the role of the transient receptor potential vanilloid-1 channel in systemic inflammation from anti-inflammatory to proinflammatory. Cell Cycle 2012;11:343-9. 
[69] Gasz B, Racz B, Roth E, Borsiczky B, Ferencz A, Tamas A, et al. Pituitary adenylate cyclase activating polypeptide protects cardiomyocytes against oxidative stress-induced apoptosis. Peptides 2006;27:87-94.

[70] Gaszner B, Kormos V, Kozicz T, Hashimoto H, Reglodi D, Helyes Z. The behavioral phenotype of pituitary adenylate-cyclase activating polypeptide-deficient mice in anxiety and depression tests is accompanied by blunted c-Fos expression in the bed nucleus of the stria terminalis, central projecting Edinger-Westphal nucleus, ventral lateral septum, and dorsal raphe nucleus. Neuroscience 2012;202:283-99.

[71] Paxinos G, Franklin KBJ. The mouse brain in stereotaxic coordinates. Second Edition ed. San Diego, CA: Academic Press; 2004.

[72] Muftuoglu MA, Aktekin A, Ozdemir NC, Saglam A. Liver injury in sepsis and abdominal compartment syndrome in rats. Surg Today 2006;36:519-24.

[73] Gavva NR, Treanor JJ, Garami A, Fang L, Surapaneni S, Akrami A, et al. Pharmacological blockade of the vanilloid receptor TRPV1 elicits marked hyperthermia in humans. Pain 2008;136:202-10.

[74] Garami A, Shimansky YP, Pakai E, Oliveira DL, Gavva NR, Romanovsky AA. Contributions of different modes of TRPV1 activation to TRPV1 antagonist-induced hyperthermia. J Neurosci 2010;30:1435-40.

[75] Pataki I, Adamik A, Jaszberenyi M, Macsai M, Telegdy G. Pituitary adenylate cyclaseactivating polypeptide induces hyperthermia in the rat. Neuropharmacology 2000;39:1303-8.

[76] Pataki I, Adamik A, Jaszberenyi M, Macsai M, Telegdy G. Involvement of transmitters in pituitary adenylate cyclase-activating polypeptide-induced hyperthermia. Regul Pept 2003;115:187-93

[77] Absood A, Chen D, Wang ZY, Hakanson R. Vascular effects of pituitary adenylate cyclase activating peptide: a comparison with vasoactive intestinal peptide. Regul Pept 1992;40:323-9.

[78] Tsueshita T, Gandhi S, Onyuksel H, Rubinstein I. Phospholipids modulate the biophysical properties and vasoactivity of PACAP-(1-38). J Appl Physiol 2002;93:1377-83. 
[79] Hawke Z, Ivanov TR, Bechtold DA, Dhillon H, Lowell BB, Luckman SM. PACAP neurons in the hypothalamic ventromedial nucleus are targets of central leptin signaling. J Neurosci 2009;29:14828-35.

[80] Inglott MA, Farnham MM, Pilowsky PM. Intrathecal PACAP-38 causes prolonged widespread sympathoexcitation via a spinally mediated mechanism and increases in basal metabolic rate in anesthetized rat. Am J Physiol Heart Circ Physiol 2011;300:H2300-7.

[81] Weinert D, Waterhouse J. Diurnally changing effects of locomotor activity on body temperature in laboratory mice. Physiol Behav 1998;63:837-43.

[82] Sagar SM, Sharp FR, Curran T. Expression of c-fos protein in brain: metabolic mapping at the cellular level. Science 1988;240:1328-31.

[83] Nakamura K, Morrison SF. A thermosensory pathway that controls body temperature. Nat Neurosci 2008;11:62-71.

[84] Osaka T. Cold-induced thermogenesis mediated by GABA in the preoptic area of anesthetized rats. Am J Physiol Regul Integr Comp Physiol 2004;287:R306-R13.

[85] Ivanov AI, Pero RS, Scheck AC, Romanovsky AA. Prostaglandin E(2)-synthesizing enzymes in fever: differential transcriptional regulation. Am J Physiol Regul Integr Comp Physiol 2002;283:R1104-17.

[86] Steiner AA, Ivanov AI, Serrats J, Hosokawa H, Phayre AN, Robbins JR, et al. Cellular and molecular bases of the initiation of fever. PLoS Biol 2006;4:e284.

[87] Kadl A, Pontiller J, Exner M, Leitinger N. Single bolus injection of bilirubin improves the clinical outcome in a mouse model of endotoxemia. Shock 2007;28:582-8.

[88] Kasap B, Soylu A, Ertoy Baydar D, Kiray M, Tugyan K, Kavukcu S. Protective effects of bilirubin in an experimental rat model of pyelonephritis. Urology 2012;80:1389 e1722.

[89] Kawamura K, Ishikawa K, Wada Y, Kimura S, Matsumoto H, Kohro T, et al. Bilirubin from heme oxygenase-1 attenuates vascular endothelial activation and dysfunction. Arterioscler Thromb Vasc Biol 2005;25:155-60.

[90] Krukow N, Brodersen R. Toxic effects in the Gunn rat of combined treatment with bilirubin and orotic acid. Acta Paediatr Scand 1972;61:697-703.

[91] Kamisaka K, Gatmaitan Z, Moore CL, Arias IM. Ligandin reverses bilirubin inhibition of liver mitochondrial respiration in vitro. Pediatr Res 1975;9:903-5. 
[92] Mustafa MG, Cowger ML, King TE. Effects of bilirubin on mitochondrial reactions. J Biol Chem 1969;244:6403-14.

[93] Noir BA, Boveris A, Garaza Pereira AM, Stoppani AO. Bilirubin: a multi-site inhibitor of mitochondrial respiration. FEBS Lett 1972;27:270-4.

[94] Cannon B, Nedergaard J. Brown adipose tissue: function and physiological significance. Physiol Rev 2004;84:277-359.

[95] Romanovsky AA, Shido O, Sakurada S, Sugimoto N, Nagasaka T. Endotoxin shock: thermoregulatory mechanisms. Am J Physiol 1996;270:R693-703.

[96] Pflueger A, Croatt AJ, Peterson TE, Smith LA, d'Uscio LV, Katusic ZS, et al. The hyperbilirubinemic Gunn rat is resistant to the pressor effects of angiotensin II. Am J Physiol Renal Physiol 2005;288:F552-8.

[97] Stec DE, Storm MV, Pruett BE, Gousset MU. Antihypertensive actions of moderate hyperbilirubinemia: role of superoxide inhibition. Am J Hypertens 2013;26:918-23.

[98] Vera T, Granger JP, Stec DE. Inhibition of bilirubin metabolism induces moderate hyperbilirubinemia and attenuates ANG II-dependent hypertension in mice. Am J Physiol Regul Integr Comp Physiol 2009;297:R738-43.

[99] Maruhashi T, Soga J, Fujimura N, Idei N, Mikami S, Iwamoto $Y$, et al. Hyperbilirubinemia, augmentation of endothelial function, and decrease in oxidative stress in Gilbert syndrome. Circulation 2012;126:598-603.

[100] Lee DH, Blomhoff R, Jacobs DR, Jr. Is serum gamma glutamyltransferase a marker of oxidative stress? Free Radic Res 2004;38:535-9.

[101] Lee DH, Gross MD, Jacobs DR, Jr. Association of serum carotenoids and tocopherols with gamma-glutamyltransferase: the Cardiovascular Risk Development in Young Adults (CARDIA) Study. Clin Chem 2004;50:582-8.

[102] Lim JS, Yang JH, Chun BY, Kam S, Jacobs DR, Jr., Lee DH. Is serum gammaglutamyltransferase inversely associated with serum antioxidants as a marker of oxidative stress? Free Radic Biol Med 2004;37:1018-23.

[103] Lanone S, Bloc S, Foresti R, Almolki A, Taille C, Callebert J, et al. Bilirubin decreases nos 2 expression via inhibition of $\mathrm{NAD}(\mathrm{P}) \mathrm{H}$ oxidase: implications for protection against endotoxic shock in rats. FASEB J 2005;19:1890-2. 
[104] Ben-Amotz R, Bonagura J, Velayutham M, Hamlin R, Burns P, Adin C. Intraperitoneal bilirubin administration decreases infarct area in a rat coronary ischemia/reperfusion model. Front Physiol 2014;5:53.

[105] Oh SW, Lee ES, Kim S, Na KY, Chae DW, Chin HJ. Bilirubin attenuates the renal tubular injury by inhibition of oxidative stress and apoptosis. BMC Nephrol 2013;14:105.

[106] Almeida MC, Steiner AA, Branco LG, Romanovsky AA. Neural substrate of coldseeking behavior in endotoxin shock. PLoS One 2006;1:e1.

[107] Almeida MC, Steiner AA, Branco LG, Romanovsky AA. Cold-seeking behavior as a thermoregulatory strategy in systemic inflammation. Eur J Neurosci 2006;23:3359-67.

[108] Liu E, Lewis K, Al-Saffar H, Krall CM, Singh A, Kulchitsky VA, et al. Naturally occurring hypothermia is more advantageous than fever in severe forms of lipopolysaccharide- and Escherichia coli-induced systemic inflammation. Am J Physiol Regul Integr Comp Physiol 2012;302:R1372-83.

[109] Romanovsky AA, Shido O, Sakurada S, Sugimoto N, Nagasaka T. Endotoxin shockassociated hypothermia. How and why does it occur? Ann N Y Acad Sci 1997;813:7337.

[110] Gunn CH. Hereditary acholuric jaundice: in a new mutant strain of rats. Journal of Heredity 1938;29:137-9.

[111] Kotal P, Van der Veere CN, Sinaasappel M, Elferink RO, Vitek L, Brodanova M, et al. Intestinal excretion of unconjugated bilirubin in man and rats with inherited unconjugated hyperbilirubinemia. Pediatr Res 1997;42:195-200.

[112] Schmid R, Hammaker L. Metabolism and Disposition of C14-Bilirubin in Congenital Nonhemolytic Jaundice. J Clin Invest 1963;42:1720-34.

[113] Iida T, Shimizu I, Nealen ML, Campbell A, Caterina M. Attenuated fever response in mice lacking TRPV1. Neurosci Lett 2005;378:28-33.

[114] Motter AL, Ahern GP. TRPV1-null mice are protected from diet-induced obesity. FEBS Lett 2008;582:2257-62.

[115] Szelenyi Z, Hummel Z, Szolcsanyi J, Davis JB. Daily body temperature rhythm and heat tolerance in TRPV1 knockout and capsaicin pretreated mice. Eur J Neurosci 2004;19:1421-4. 
[116] Wang Y, Novotny M, Quaiserova-Mocko V, Swain GM, Wang DH. TRPV1-mediated protection against endotoxin-induced hypotension and mortality in rats. Am J Physiol Regul Integr Comp Physiol 2008;294:R1517-R23.

[117] Wanner SP, Garami A, Romanovsky AA. Hyperactive when young, hypoactive and overweight when aged: connecting the dots in the story about locomotor activity, body mass, and aging in Trpv1 knockout mice. Aging (Albany NY) 2011;3:450-4.

[118] Steiner AA, Turek VF, Almeida MC, Burmeister JJ, Oliveira DL, Roberts JL, et al. Nonthermal activation of transient receptor potential vanilloid-1 channels in abdominal viscera tonically inhibits autonomic cold-defense effectors. J Neurosci 2007;27:745968.

[119] Steiner AA, Oliveira DL, Roberts JL, Petersen SR, Romanovsky AA. Nicotine administration and withdrawal affect survival in systemic inflammation models. J Appl Physiol 2008;105:1028-34.

[120] Cross A, Asher L, Seguin M, Yuan L, Kelly N, Hammack C, et al. The importance of a lipopolysaccharide-initiated, cytokine-mediated host defense mechanism in mice against extraintestinally invasive Escherichia coli. J Clin Invest 1995;96:676-86.

[121] Cross AS, Sadoff JC, Kelly N, Bernton E, Gemski P. Pretreatment with recombinant murine tumor necrosis factor alpha/cachectin and murine interleukin 1 alpha protects mice from lethal bacterial infection. J Exp Med 1989;169:2021-7.

[122] Vazquez-Torres A, Vallance BA, Bergman MA, Finlay BB, Cookson BT, Jones-Carson $\mathrm{J}$, et al. Toll-like receptor 4 dependence of innate and adaptive immunity to Salmonella: importance of the Kupffer cell network. J Immunol 2004;172:6202-8.

[123] Ang SF, Moochhala SM, Bhatia M. Hydrogen sulfide promotes transient receptor potential vanilloid 1-mediated neurogenic inflammation in polymicrobial sepsis. Crit Care Med 2010;38:619-28.

[124] Murai M, Tsuji F, Nose M, Seki I, Oki K, Setoguchi C, et al. SA13353 (1-[2-(1Adamantyl)ethyl]-1-pentyl-3-[3-(4-pyridyl)propyl]urea) inhibits TNF-alpha production through the activation of capsaicin-sensitive afferent neurons mediated via transient receptor potential vanilloid 1 in vivo. Eur J Pharmacol 2008;588:309-15.

[125] Wang S, Davis BM, Zwick M, Waxman SG, Albers KM. Reduced thermal sensitivity and Nav1.8 and TRPV1 channel expression in sensory neurons of aged mice. Neurobiol Aging 2006;27:895-903. 
[126] Bergman E, Fundin BT, Ulfhake B. Effects of aging and axotomy on the expression of neurotrophin receptors in primary sensory neurons. J Comp Neurol 1999;410:368-86.

[127] Marik PE, Zaloga GP. The effect of aging on circulating levels of proinflammatory cytokines during septic shock. Norasept II Study Investigators. J Am Geriatr Soc 2001;49:5-9.

[128] Stewart L, Grifiss JM, Jarvis GA, Way LW. Elderly patients have more severe biliary infections: influence of complement-killing and induction of TNFalpha production. Surgery 2008;143:103-12.

[129] Watanabe E, Hirasawa H, Oda S, Matsuda K, Hatano M, Tokuhisa T. Extremely high interleukin-6 blood levels and outcome in the critically ill are associated with tumor necrosis factor- and interleukin-1-related gene polymorphisms. Crit Care Med 2005;33:89-97; discussion 242-3.

[130] Banks WA, Kastin AJ, Komaki G, Arimura A. Passage of pituitary adenylate cyclase activating polypeptide1-27 and pituitary adenylate cyclase activating polypeptide1-38 across the blood-brain barrier. J Pharmacol Exp Ther 1993;267:690-6.

[131] Nonaka N, Banks WA, Mizushima H, Shioda S, Morley JE. Regional differences in PACAP transport across the blood-brain barrier in mice: a possible influence of strain, amyloid beta protein, and age. Peptides 2002;23:2197-202.

[132] Romanovsky AA. Temperature regulation. Chapter 23. In: Petersen O, editor. Lecture Notes on Human Physiology, 5th Edition. Oxford: Blackwell; 2007. p. 603-15.

[133] Nakamura K, Morrison SF. Preoptic mechanism for cold-defensive responses to skin cooling. J Physiol 2008;586:2611-20.

[134] Hashimoto H, Shintani N, Tanaka K, Mori W, Hirose M, Matsuda T, et al. Altered psychomotor behaviors in mice lacking pituitary adenylate cyclase-activating polypeptide (PACAP). Proc Natl Acad Sci U S A 2001;98:13355-60.

[135] Adams BA, Gray SL, Isaac ER, Bianco AC, Vidal-Puig AJ, Sherwood NM. Feeding and metabolism in mice lacking pituitary adenylate cyclase-activating polypeptide. Endocrinology 2008;149:1571-80.

[136] Weinert D, Waterhouse J. Daily activity and body temperature rhythms do not change simultaneously with age in laboratory mice. Physiol Behav 1999;66:605-12. 
[137] Hashimoto H, Hashimoto R, Shintani N, Tanaka K, Yamamoto A, Hatanaka M, et al. Depression-like behavior in the forced swimming test in PACAP-deficient mice: amelioration by the atypical antipsychotic risperidone. J Neurochem 2009;110:595-602.

[138] Kanizsai P, Garami A, Solymar M, Szolcsanyi J, Szelenyi Z. Energetics of fasting heterothermia in TRPV1-KO and wild type mice. Physiol Behav 2009;96:149-54.

[139] Szentirmai E, Kapas L, Sun Y, Smith RG, Krueger JM. Restricted feeding-induced sleep, activity, and body temperature changes in normal and preproghrelin-deficient mice. Am J Physiol Regul Integr Comp Physiol 2010;298:R467-77.

[140] Brito HO, Barbosa FL, Reis RC, Fraga D, Borges BS, Franco CR, et al. Evidence of substance P autocrine circuitry that involves TNF-alpha, IL-6, and PGE2 in endogenous pyrogen-induced fever. J Neuroimmunol 2016;293:1-7.

[141] Engstrom L, Ruud J, Eskilsson A, Larsson A, Mackerlova L, Kugelberg U, et al. Lipopolysaccharide-induced fever depends on prostaglandin E2 production specifically in brain endothelial cells. Endocrinology 2012;153:4849-61.

[142] Eskilsson A, Mirrasekhian E, Dufour S, Schwaninger M, Engblom D, Blomqvist A. Immune-induced fever is mediated by IL-6 receptors on brain endothelial cells coupled to STAT3-dependent induction of brain endothelial prostaglandin synthesis. J Neurosci 2014;34:15957-61.

[143] Rummel C, Matsumura K, Luheshi GN. Circulating IL-6 contributes to peripheral LPSinduced mPGES-1 expression in the rat brain. Brain Res Bull 2011;86:319-25.

[144] Rummel C, Sachot C, Poole S, Luheshi GN. Circulating interleukin-6 induces fever through a STAT3-linked activation of COX-2 in the brain. Am J Physiol Regul Integr Comp Physiol 2006;291:R1316-26.

[145] Lotz M, Vaughan JH, Carson DA. Effect of neuropeptides on production of inflammatory cytokines by human monocytes. Science 1988;241:1218-21.

[146] Boichot E, Germain N, Emonds-Alt X, Advenier C, Lagente V. Effects of SR 140333 and SR 48968 on antigen and substance P-induced activation of guinea-pig alveolar macrophages. Clin Exp Allergy 1998;28:1299-305.

[147] Sio SW, Ang SF, Lu J, Moochhala S, Bhatia M. Substance P upregulates cyclooxygenase-2 and prostaglandin E metabolite by activating ERK1/2 and NFkappaB in a mouse model of burn-induced remote acute lung injury. J Immunol 2010;185:6265-76. 
[148] Bang R, Sass G, Kiemer AK, Vollmar AM, Neuhuber WL, Tiegs G. Neurokinin-1 receptor antagonists CP-96,345 and L-733,060 protect mice from cytokine-mediated liver injury. J Pharmacol Exp Ther 2003;305:31-9.

[149] Bost KL, Breeding SA, Pascual DW. Modulation of the mRNAs encoding substance P and its receptor in rat macrophages by LPS. Reg Immunol 1992;4:105-12.

[150] Ho WZ, Lai JP, Zhu XH, Uvaydova M, Douglas SD. Human monocytes and macrophages express substance $\mathrm{P}$ and neurokinin-1 receptor. $\mathrm{J}$ Immunol 1997;159:5654-60.

[151] Klassert TE, Pinto F, Hernandez M, Candenas ML, Hernandez MC, Abreu J, et al. Differential expression of neurokinin B and hemokinin-1 in human immune cells. J Neuroimmunol 2008;196:27-34.

[152] Lai JP, Douglas SD, Ho WZ. Human lymphocytes express substance P and its receptor. J Neuroimmunol 1998;86:80-6.

[153] Marriott I, Bost KL. IL-4 and IFN-gamma up-regulate substance P receptor expression in murine peritoneal macrophages. J Immunol 2000;165:182-91.

[154] Steinhoff MS, von Mentzer B, Geppetti P, Pothoulakis C, Bunnett NW. Tachykinins and their receptors: contributions to physiological control and the mechanisms of disease. Physiol Rev 2014;94:265-301.

[155] Barrios-Rodiles M, Tiraloche G, Chadee K. Lipopolysaccharide modulates cyclooxygenase- 2 transcriptionally and posttranscriptionally in human macrophages independently from endogenous IL-1 beta and TNF-alpha. J Immunol 1999;163:963-9.

[156] Maier T, Guell M, Serrano L. Correlation of mRNA and protein in complex biological samples. FEBS Lett 2009;583:3966-73.

[157] Park YK, Hong H, Jang BC. Transcriptional and translational regulation of COX-2 expression by cadmium in C6 glioma cells. Int J Mol Med 2012;30:960-6.

[158] Ristimaki A, Garfinkel S, Wessendorf J, Maciag T, Hla T. Induction of cyclooxygenase2 by interleukin-1 alpha. Evidence for post-transcriptional regulation. J Biol Chem 1994;269:11769-75.

[159] Oka T, Oka K, Kobayashi T, Sugimoto Y, Ichikawa A, Ushikubi F, et al. Characteristics of thermoregulatory and febrile responses in mice deficient in prostaglandin EP1 and EP3 receptors. J Physiol 2003;551:945-54. 
[160] Romanovsky AA, Ivanov AI, Karman EK. Blood-borne, albumin-bound prostaglandin E2 may be involved in fever. Am J Physiol 1999;276:R1840-4.

[161] Garami A, Pakai E, McDonald HA, Reilly RM, Gomtsyan A, Corrigan JJ, et al. TRPV1 antagonists that cause hypothermia, instead of hyperthermia, in rodents: Compounds' pharmacological profiles, in vivo targets, thermoeffectors recruited and implications for drug development. Acta Physiol (Oxf) 2018;223:e13038.

[162] Garami A, Shimansky YP, Rumbus Z, Vizin RCL, Farkas N, Hegyi J, et al. Hyperthermia induced by transient receptor potential vanilloid-1 (TRPV1) antagonists in human clinical trials: Insights from mathematical modeling and meta-analysis. Pharmacol Ther 2020;208:107474.

[163] Moran MM, McAlexander MA, Biro T, Szallasi A. Transient receptor potential channels as therapeutic targets. Nat Rev Drug Discov 2011;10:601-20.

[164] High KP, Bradley SF, Gravenstein S, Mehr DR, Quagliarello VJ, Richards C, et al. Clinical practice guideline for the evaluation of fever and infection in older adult residents of long-term care facilities: 2008 update by the Infectious Diseases Society of America. J Am Geriatr Soc 2009;57:375-94.

[165] Norman DC. Fever in the elderly. Clin Infect Dis 2000;31:148-51.

[166] Jones II AJ, (contact). Vanda Pharmaceuticals announces the initiation of ODYSSEY, an FDA approved clinical study of Tradipitant in hospitalized patients with severe COVID-19 pneumonia: https://vandapharmaceuticalsinc.gcs-web.com/node/14056/pdf2020 [June 30, 2020]. 
APPENDICES 


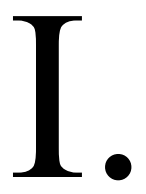




\section{OPEN ACCESS}

Edited by:

Christoph Thiemermann, Queen Mary University of London, United Kingdom

Reviewed by: Hugo Caire Castro-Faria-Neto, Fundação Oswaldo Cruz (Fiocruz), Brazil Dianne Cooper, Queen Mary University of London, United Kingdom

*Correspondence: Andras Garami andras.garami@aok.pte.hu

Specialty section: This article was submitted to Inflammation, a section of the journal Frontiers in Immunology

Received: 17 November 2017 Accepted: 18 January 2018 Published: 05 February 2018

Citation:

Pakai E, Tekus V, Zsiboras C, Rumbus Z, Olah E, Keringer $P$, Khidhir N, Matics R, Deres L, Ordog K, Szentes N, Pohoczky K, Kemeny A, Hegyi P, Pinter E and Garami A (2018) The Neurokinin-1 Receptor Contributes to the Early Phase of Lipopolysaccharide-Induced Fever via Stimulation of Peripheral Cyclooxygenase-2 Protein Expression in Mice.

Front. Immunol. 9:166. doi: 10.3389/fimmu.2018.00166
The Neurokinin-1 Receptor Contributes to the Early Phase of Lipopolysaccharide-Induced Fever via Stimulation of Peripheral Cyclooxygenase-2 Protein Expression in Mice

Eszter Pakai ${ }^{1,2}$, Valeria Tekus ${ }^{3,4}$, Csaba Zsiboras', Zoltan Rumbus', Emoke Olah', Patrik Keringer ${ }^{1}$, Nora Khidhir', Robert Matics' ${ }^{1}$, Laszlo Deres ${ }^{4,5}$, Katalin Ordog ${ }^{4,5}$, Nikolett Szentes ${ }^{3,4}$, Krisztina Pohoczky ${ }^{3,4}$, Agnes Kemeny ${ }^{4,6}$, Peter Hegyi ${ }^{1,2,7}$, Erika Pinter ${ }^{3,4}$ and Andras Garami ${ }^{1 *}$

${ }^{1}$ Institute for Translational Medicine, Medical School, University of Pecs, Pecs, Hungary, ${ }^{2}$ Momentum Gastroenterology Multidisciplinary Research Group, Hungarian Academy of Sciences - University of Szeged, Szeged, Hungary, ${ }^{3}$ Department of Pharmacology and Pharmacotherapy, Medical School, University of Pecs, Pecs, Hungary, ${ }^{4}$ Janos Szentagothai Research Centre, University of Pecs, Pecs, Hungary, ${ }^{5}$ First Department of Medicine, Medical School, University of Pecs, Pecs, Hungary, ${ }^{6}$ Department of Medical Biology, Medical School, University of Pecs, Pecs, Hungary, ${ }^{7}$ First Department of Medicine, University of Szeged, Szeged, Hungary

Neurokinin (NK) signaling is involved in various inflammatory processes. A common manifestation of systemic inflammation is fever, which is usually induced in animal models with the administration of bacterial lipopolysaccharide (LPS). A role for the NK1 receptor was shown in LPS-induced fever, but the underlying mechanisms of how the NK1 receptor contributes to febrile response, especially in the early phase, have remained unknown. We administered LPS (120 $\mu \mathrm{g} / \mathrm{kg}$, intraperitoneally) to mice with the Tacr1 gene, i.e., the gene encoding the NK1 receptor, either present $\left(\operatorname{TaCr}^{1+/+}\right)$ or absent $\left(\operatorname{TaCr}^{1-1^{--}}\right)$and measured their thermoregulatory responses, serum cytokine levels, tissue cyclooxygenase-2 (COX-2) expression, and prostaglandin (PG) $\mathrm{E}_{2}$ concentration. We found that the LPS-induced febrile response was attenuated in $\mathrm{TaCr}^{-{ }^{--}}$compared to their TaCr1+/+ littermates starting from 40 min postinfusion. The febrigenic effect of intracerebroventricularly administered $\mathrm{PGE}_{2}$ was not suppressed in the Tacr1 ${ }^{-1-}$ mice. Serum concentration of pyrogenic cytokines did not differ between $\mathrm{TaCr}^{-1_{-}^{-}}$and $\mathrm{TaCr}^{+{ }^{++}}$at 40 min post-LPS infusion. Administration of LPS resulted in amplification of COX-2 mRNA expression in the lungs, liver, and brain of the mice, which was statistically indistinguishable between the genotypes. In contrast, the LPS-induced augmentation of COX-2 protein expression was attenuated in the lungs and tended to be suppressed in the liver of $\mathrm{TaCr}^{-1-}$ mice compared with $\mathrm{TaCr} 1^{+/+}$mice. The Tacr ${ }^{+/+}$mice responded to LPS with a significant surge of $\mathrm{PGE}_{2}$ production in the lungs, whereas Tacr1 ${ }^{-1-}$ mice did not. In conclusion, the NK1 receptor is necessary for normal fever genesis. Our results suggest that the NK1 receptor contributes to the early phase of LPS-induced fever by enhancing COX-2 protein expression in the periphery. These findings advance the understanding of the crosstalk between NK signaling and the "cytokine-COX-2-prostaglandin $\mathrm{E}_{2}$ " axis in systemic inflammation, thereby open up the possibilities for new therapeutic approaches.

Keywords: fever, thermoregulation, systemic inflammation, endotoxin, cyclooxygenase, autonomic thermoeffectors, substance $P$, Tacr1 


\section{INTRODUCTION}

The neurokinin-1 (NK1) receptor, formerly also known as substance $\mathrm{P}(\mathrm{SP})$ receptor, plays an important role in mediation of local and systemic inflammatory processes (1). As part of systemic inflammation the most often developing thermoregulatory response is fever, which is commonly induced in experimental animals by the administration of bacterial lipopolysaccharide (LPS). In the development of the febrile response to LPS several molecular mechanisms have been already identified $(2,3)$. In brief, LPS triggers the activation of peripheral macrophages, which then produce inflammatory cytokines such as interleukin (IL)- $1 \beta$, IL-6, and tumor necrosis factor (TNF)- $\alpha$ and induce the activation of the arachidonic acid cascade. Arachidonic acid is produced from membrane phospholipids by the action of phospholipases $\mathrm{A}_{2}\left[\mathrm{PLA}_{2}\right.$; reviewed in Ref. (4)] and via alternative pathways, such as monoacylglycerol lipase-dependent hydrolysis (5). In the next step of febrigenesis, the cyclooxygenase-2 (COX2) enzyme is of crucial importance as it has been shown that selective blockade of COX-2 completely abolishes the fever response $(6,7)$. Among the end products of the cascade, prostaglandin (PG) $\mathrm{E}_{2}$ is synthesized by terminal PGE synthases, which can be microsomal and cytosolic (8). $\mathrm{PGE}_{2}$ is a key mediator, since it is produced in the periphery already in the early stage of fever (9) and because its binding to EP3 receptors in the hypothalamus triggers the activation of thermogenesis and cutaneous vasoconstriction, thereby resulting in fever $(10,11)$. In addition to the aforementioned mechanisms, various further substances have been identified as mediators of fever, which also include SP signaling $(3,12)$.

Indicating the role of SP in fever, when the effects of SP were antagonized with peptideSP analogs, the fever response to LPS was blocked in guinea pigs (13) and in rats (14). Similar attenuation of the LPS-induced fever was observed in rats after administration of the NK1 receptor antagonists CP-96,345 (15) and SR140333B (16). These studies strongly support that SP signaling contributes to the development of LPS-induced fever, but it has remained largely unknown which mediators of the febrile process are influenced by SP or its receptors. In all of these studies, the authors used antagonists, which can be problematic because of their short half-lives, poor brain penetration, and off-target effects (17). In an earlier study, SP inhibited pancreatic bicarbonate secretion via NK2 and NK3 receptors (18), suggesting that the effects of SP or its peptide analog antagonists are not solely mediated by NK1 receptors. In the case of the non-peptide antagonists, it was shown that at higher doses CP-96,345 and SR140333B also block L-type calcium channels $(19,20)$. Alternative approaches, such as the use of knockout mice, can help to complement the findings with antagonists about the contribution of the NK1 receptor to

\footnotetext{
Abbreviations: COX, cyclooxygenase; GAPDH, glyceraldehyde 3-phosphate dehydrogenase; GM-CSF, granulocyte-macrophage colony-stimulating factor; i.c.v., intracerebroventricular(ly); i.p., intraperitoneal(ly); IL, interleukin; LPS, lipopolysaccharide; NK, neurokinin; $\mathrm{PG}$, prostaglandin; $\mathrm{PKC}$, protein kinase $\mathrm{C}$; $\mathrm{PLA}_{2}$, phospholipase $\mathrm{A}_{2}$ (e.g., cytosolic, $\mathrm{CPLA}_{2}$ ); SP, substance $\mathrm{P} ; \mathrm{T}_{\mathrm{b}}$, body temperature; $T_{\text {sk }}$, skin temperature; $\mathrm{PE}$, polyethylene; $\mathrm{TNF}$, tumor necrosis factor; $\mathrm{VO}_{2}$, rate of oxygen consumption.
}

fever. In addition to complementing the earlier findings with antagonists, by using knockout mice our main goal was to better clarify which step(s) of the classical molecular mechanisms of fever are influenced by the NK1 receptor.

In the present work, we studied how genetic ablation of the NK1 receptor influences the LPS-induced fever response in mice. In thermophysiological experiments, we recorded changes in deep body temperature $\left(T_{\mathrm{b}}\right)$ and in the activity of autonomic thermoeffectors in response to LPS and $\mathrm{PGE}_{2}$. To identify the involved molecular mechanisms, we measured serum cytokine levels, as well as tissue COX-2 mRNA and protein expression, and $\mathrm{PGE}_{2}$ concentration in the same animal model.

\section{MATERIALS AND METHODS}

\section{Animals}

The experiments were performed in 174 adult mice of both sexes. To minimize the possibility that gender of the mice has an influence on our results, male and female mice were approximately equally distributed in age-matched experimental groups. Of the mice, 86 had the Tacrl gene, i.e., the gene encoding the NK1 receptor, homozygously present $\left(\mathrm{TaCr}^{+/+}\right)$, while 88 absent $\left(\mathrm{Tacrl}^{-/-}\right)$due to a targeted disruption (21). The $\mathrm{Tacrl}^{-/-}$mice were generated at the University of Liverpool as described in detail elsewhere (21). The original breeding pairs of the Tacr1 ${ }^{-/-}$ mice were donated to the University of Pecs by Dr. John Quinn (University of Liverpool). Their breeding and backcrossing on a C57BL/6 background (for at least 10 generations) were reported in our recent study (22). The mice were housed in standard plastic cages kept in a room with an ambient temperature maintained at $25-27^{\circ} \mathrm{C}$ and with a humidity of $30-40 \%$. The room was on a $12 \mathrm{~h}$ light-dark cycle (lights on at 5:00 a.m.). Standard rodent chow and tap water were available ad libitum. At the time of the experiments, the Tacrl ${ }^{+/+}$and $\mathrm{Tacrl}^{-/-}$mice weighed $21 \pm 2$ and $19 \pm 2$ g, respectively.

The mice were extensively handled and then habituated to staying inside wire-mesh cylindrical confiners. The cylindrical confiner prevented the animal from turning around, but allowed for some back-and-forth movements; it was used in the thermocouple and respirometry setups (see Experimental Setups).

All procedures were conducted under protocols approved by Institutional Animal Use and Care Committee of the University of Pecs and were in accordance with the directives of the National Ethical Council for Animal Research and those of the European Communities Council (86/609/EEC).

\section{Surgeries}

Mice were anesthetized with the intraperitoneal (i.p.) administration of a ketamine-xylazine cocktail ( 81.7 and $9.3 \mathrm{mg} / \mathrm{kg}$, respectively) and received antibiotic protection intramuscularly (gentamycin, $6 \mathrm{mg} / \mathrm{kg}$ ). During surgery, a mouse was heated with a temperature-controlled heating pad (model TMP-5a; Supertech Instruments UK Ltd., London, UK) placed under a surgery board. For pain management, ketoprofen $(5 \mathrm{mg} / \mathrm{kg})$ was administered subcutaneously at the end of surgery and on the next day. The experiments were performed 4-7 days after the surgery. 
For i.p. catheter implantation, a small midline incision was made on the abdominal wall, and then a polyethylene (PE)-50 catheter filled with pyrogen-free saline was inserted into the peritoneal cavity. The internal end of the catheter was fixed to the left side of the abdominal wall with a suture; the free end of the catheter was tunneled under the skin to the nape where it was exteriorized and heat-sealed. The surgical wound was sutured in layers. The catheter was flushed with $0.1 \mathrm{ml}$ of saline on the day after the surgery and every other day thereafter. No sign of discomfort or inflammation was associated with this procedure in the present study and in our other studies in mice $(23,24)$ and in rats $(24,25)$.

For intracerebroventricular (i.c.v.) cannula implantation, each mouse was fixed to a stereotaxic apparatus similarly as in our earlier study (26). The scalp was incised over the sagittal suture; the periosteum was excised; the skull was cleaned and dried; two supporting microscrews were driven into the skull; and a small hole was drilled in the skull $0.5 \mathrm{~mm}$ posterior from bregma and $1.0 \mathrm{~mm}$ lateral from midline. A 22-G steel guide cannula (Plastics One, Roanoke, VA, USA) was attached to a plastic tube fitted into a stereotaxic manipulator (Narishige Scientific Instruments Laboratory, Tokyo, Japan), which was used to insert the cannula into the brain through the bone hole. The tip of the cannula was placed within the right lateral ventricle $(2.0 \mathrm{~mm}$ from dura). The cannula was secured to the supporting microscrews with dental cement and released from the manipulator. The guide cannula was closed by a dummy cannula.

\section{Experimental Setups}

The thermophysiological experiments were performed in the thermocouple or in the respirometry setup.

In the thermocouple setup, the mouse was placed in a cylindrical confiner and equipped with copper-constantan thermocouples (Omega Engineering, Stamford, CT, USA) to measure colonic temperature (a form of deep $T_{\mathrm{b}}$ ) and tail skin temperature $\left(T_{\mathrm{sk}}\right)$. The colonic thermocouple was inserted $3 \mathrm{~cm}$ deep beyond the anal sphincter and was fixed to the base of the tail with a loop of adhesive tape. The skin thermocouple was positioned on the lateral surface of tail at the border between the proximal and middle third of the tail and secured in place with tape. The thermocouples were plugged into a data logger device (Cole-Palmer, Vernon Hills, IL, USA) connected to a computer. Mice in their confiners were then placed into a temperature-controlled incubator (model MIDI F230S; PL Maschine Ltd., Tarnok, Hungary) set to an ambient temperature of $31^{\circ} \mathrm{C}$, which is at the lower end of the thermoneutral zone for mice in this setup. When present, the i.p. catheter was connected to a PE-50 extension filled with the drug of interest. When the mouse had an i.c.v. cannula, a needle injector was fitted into the guide cannula and connected to a PE-50 extension. The extension was passed through a port of the chamber and connected to a syringe. In a separate set of experiments, the mice were exposed to heat or cold by applying similar protocols as in our earlier studies $(26,27)$. The mice in their confiners were placed into a biochemistry incubator (model BJPX-Newark; Biobase, Jinan, China) initially set to an ambient temperature of $33^{\circ} \mathrm{C}$, which was then either raised to $39^{\circ} \mathrm{C}$ or decreased to $15^{\circ} \mathrm{C}$ over $\sim 30 \mathrm{~min}$ and maintained at 39 or $15^{\circ} \mathrm{C}$ to expose the mice to heat or cold, respectively.
A mouse designated for an experiment in the respirometry setup was equipped with thermocouples and placed in a confiner as in experiments in the thermocouple setup. Then, the mouse in its confiner was transferred to a Plexiglas chamber of the four-chamber open-circuit calorimeter integrated system (Oxymax Equal Flow, Columbus Instruments), as in our earlier studies $(25,27)$. The chamber was sealed, submerged into a temperature-controlled $\left(31^{\circ} \mathrm{C}\right)$ water bath, and continuously ventilated with room air $(200 \mathrm{ml} / \mathrm{min})$. The fractional concentration of oxygen was measured in the air entering and exiting the chamber, and the rate of oxygen consumption $\left(\mathrm{VO}_{2}\right)$ was calculated according to the manufacturer's instructions using the Oxymax Windows software (version 3.1). The extension of the i.p. catheter or the i.c.v. needle injector was passed through a port of the chamber and connected to a syringe, which was placed in a syringe pump (model 975; Harvard Apparatus Inc., Holliston, MA, USA).

\section{Substance Administration}

Lipopolysaccharide from Escherichia coli 0111:B4 was purchased from Sigma-Aldrich (St. Louis, MO, USA). A stock suspension of LPS $(5 \mathrm{mg} / \mathrm{ml})$ in pyrogen-free saline was stored at $-20^{\circ} \mathrm{C}$. On the day of the experiment, the stock was diluted to a final concentration of $36 \mu \mathrm{g} / \mathrm{ml}$. The diluted LPS suspension or saline was infused $(26 \mu \mathrm{l} / \mathrm{min}$ for $4 \mathrm{~min})$ through the extension of the i.p. catheter to deliver LPS at a final dose of $120 \mu \mathrm{g} / \mathrm{kg}$. Deep $T_{\mathrm{b}}$, tail $T_{\mathrm{sk}}$, and $\mathrm{VO}_{2}$ were monitored for $6 \mathrm{~h}$ after the injection.

Substance $\mathrm{P}$ and $\mathrm{PGE}_{2}$ were purchased from Tocris Bioscience (Bristol, UK). Aliquots of ethanolic stock solutions of SP and $\mathrm{PGE}_{2}$ (10 and $12.5 \mathrm{mg} / \mathrm{ml}$, respectively) were stored at $-80^{\circ} \mathrm{C}$. On the day of the experiment, the stock solutions were diluted with ethanol and saline to give working solutions of SP and $\mathrm{PGE}_{2}$ ( 1,000 and $33 \mu \mathrm{g} / \mathrm{ml}$, respectively) in $10 \%$ ethanol. By infusing these solutions into the lateral ventricle $(1 \mu \mathrm{l} / \mathrm{min}$ for $3 \mathrm{~min})$, a total dose of either $100 \mu \mathrm{g} / \mathrm{kg}$ SP or $3.3 \mu \mathrm{g} / \mathrm{kg} \mathrm{PGE}_{2}$ was delivered i.c.v. Control mice were infused with the vehicle ( $10 \%$ ethanol in saline). For the infusion, the dummy injector was removed from the preimplanted guide cannula and replaced with a $28-\mathrm{G}$ injector needle (Plastics One) connected to a $10-\mu$ l Hamilton syringe by a PE-50 extension. The injector needle protruded $1.0 \mathrm{~mm}$ beyond the tip of the guide cannula.

Administration of the substances was carried out between 10:30 a.m. and 12:00 p.m. in the experiments.

\section{Molecular Biology Tissue Harvesting}

Each mouse was implanted with an i.p. catheter and extensively adapted to the experimental setup. On the day of experiment, each mouse was placed in a confiner and transferred to an incubator chamber, which was set to an ambient temperature of $31^{\circ} \mathrm{C}$. The i.p. catheter was connected to a PE-50 extension filled with LPS or saline. The extension was passed through a port of the chamber and connected to a syringe, which was placed in a syringe pump (Harvard Apparatus Inc.). Mice were left to acclimate for $\sim 2 \mathrm{~h}$ and then infused with LPS or saline as in the thermophysiological experiments. Forty minutes after infusion, the mice were anesthetized with ketamine-xylazine cocktail, 
which was injected through the extension of the i.p. catheter. Blood samples were collected from the left ventricle. Each sample was transferred to an ice-cold Eppendorf tube containing EDTA $(40 \mu \mathrm{l})$ and trasylol $(20 \mu \mathrm{l})$. The collected blood was immediately centrifuged at $1,000 \mathrm{rpm}$ for $5 \mathrm{~min}$, then at $4,000 \mathrm{rpm}$ for $10 \mathrm{~min}$, and the resulting plasma was stored at $-80^{\circ} \mathrm{C}$. For collection of lung, liver, and brain tissue samples for RT-qPCR, Western blot, and immunoassay protocols, each mouse was perfused through the left ventricle with $0.1 \mathrm{M}$ phosphate-buffered saline. Samples of the liver and the right lung were collected rapidly and snap frozen in liquid nitrogen. The anesthetized mouse was decapitated, its entire brain was removed and frozen. All tissue samples were stored at $-80^{\circ} \mathrm{C}$.

\section{Immunoassays}

The serum level of TNF- $\alpha$ was determined with enzyme-linked immunosorbent assay (ELISA) by using a commercially available mouse TNF ELISA kit (BD OptEIA catalog nr: 560478; BD Biosciences, San Jose, CA, USA), which had a detection limit of $31 \mathrm{pg} / \mathrm{ml}$ for TNF- $\alpha$. PGE 2 concentrations in the lungs, liver, and brain were measured by ELISA using a commercially available kit (catalog nr: 514010; Cayman Chemical, Ann Arbor, MI, USA); the samples were prepared according to the manufacturer's instructions. The assay had a sensitivity of $15 \mathrm{pg} / \mathrm{ml}$ for $\mathrm{PGE}_{2}$. Detections were performed by using the Labsystem Multiskan RC plate reader (Thermo Scientific, Waltham, MA, USA), as in our earlier study (22).

Serum concentrations of granulocyte-macrophage colonystimulating factor (GM-CSF) and IL-6 were determined by Luminex’s $\mathrm{xMAP}^{\circledR}$ Technology using a multiplex bead immunoassay kit (catalog nr: LMC0003; Invitrogen, Carlsbad, CA, USA). After thawing, serum samples were centrifuged $(1,000 \mathrm{~g}$ for $10 \mathrm{~min}$ ) to prevent clogging of the filter plate. The measurement was performed according to the manufacturer's instructions. Following previous optimizations, all samples were tested undiluted in a blind fashion. Luminex 100 (Luminex Corporation, Austin, TX, USA) was used for the immunoassay and Luminex 100 IS software to analyze the bead median fluorescence intensity, as in our recent study (28). All the tests were run in duplicate. $50 \mu \mathrm{l}$ of each sample or standard solution was added to a 96-well filter plate (provided with the kit) containing $25 \mu \mathrm{l}$ of antibodycoated fluorescent beads. Biotinylated secondary antibodies and streptavidin-PE were added to the samples with alternate incubation and washing steps. After the last washing, $100 \mu \mathrm{l}$ of working wash solution was added to the wells. The plate was read on the Luminex 100 array reader. The detection limits for IL-6 and GM-CSF with this method were 29 and $18 \mathrm{pg} / \mathrm{ml}$, respectively. Data were analyzed with the MasterPlex 2.0 software, using a five-PL regression curve to plot the standard curve.

\section{RNA Isolation, RT-qPCR}

As in previous studies by our group $(28,29)$, total RNA was prepared using the TRI Reagent (Molecular Research Center, Inc., Cincinnati, OH, USA) and Direct-ZolTM RNA isolation kit (Zymo Research, Irvine, CA, USA) following the manufacturer's instructions. Then, the samples were treated with DNase I (Zymo Research, Irvine, CA, USA) to remove contaminating genomic DNA and quantified with a NanoDrop ND-2000 spectrophotometer (NanoDrop Technologies, Wilmington, DE, USA). One microgram of total RNA was reverse transcribed with a Maxima ${ }^{\mathrm{TM}}$ First Strand cDNA Synthesis Kit for RT-qPCR (Thermo Scientific). Reactions were performed on Stratagene Mx3000P QPCR System (Agilent Technologies, Santa Clara, CA, USA), using glyceraldehyde 3-phosphate dehydrogenase $(\mathrm{GAPDH})$ as the reference gene. GAPDH was chosen because of its stable expression and because it was successfully used in earlier studies to measure relative COX-2 mRNA expression in LPSinduced fever (30-32). Each reaction contained $2 \mu \mathrm{l}$ of cDNA, $10 \mu \mathrm{l}$ Luminaris Color HiGreen Low ROX qPCR Master Mix (Thermo Scientific), $0.3 \mu \mathrm{M}$ of each primer $(10 \mu \mathrm{M})$, and $6.8 \mu \mathrm{l}$ of water. The following primer pairs were used to amplify the target loci: GAPDH sense: 5'-TTCACCACCATGGAGAAG-3' and antisense: 5'-GGCATGGACTGTGGTCATGA-3'. COX-2 sense: 5'-GGGTTGCTGGGGGAAGAAA-3' and antisense: 5'CTCTGCTCTGGTCAATGGAGG-3'. Amplification was carried out under the following conditions: $95^{\circ} \mathrm{C}$ for $10 \mathrm{~min}$, followed by 40 cycles of $95^{\circ} \mathrm{C}$ for $30 \mathrm{~s}, 60^{\circ} \mathrm{C}$ for $45 \mathrm{~s}$, and $72^{\circ} \mathrm{C}$ for $45 \mathrm{~s}$. All RT-PCR reactions were carried out in triplicate and included a melt curve analysis to ensure specificity of signal. Relative expression ratios were calculated using MxPro QPCR Software (Agilent Technologies) with the $\Delta \Delta$ Ct method, using samples of untreated animals, as a control. Primer efficiencies were taken into account when calculating gene expression ratios (33).

\section{Western Blot}

Western blot procedures were carried out according to the protocols used in our recent study (34). Lung, liver, and brain tissue samples $(\sim 50 \mathrm{mg})$ were homogenized in ice-cold Tris buffer (50 mM, pH 8.0), containing protease inhibitor cocktail (1:100) and $50 \mathrm{mM}$ of sodium vanadate (Sigma-Aldrich).

Brain samples required additional preparation procedures. To each brain sample, $625 \mu \mathrm{l}$ of a methanol:chloroform (2:1) mixture was added, and the samples were incubated for $10 \mathrm{~min}$ at room temperature with slight agitation. Then, without inhibitors an equal volume $(208 \mu \mathrm{l})$ of chloroform and Tris buffer $(50 \mathrm{mM})$ was added to each brain sample, and the samples were centrifuged for $10 \mathrm{~min}(13,000 \mathrm{rpm}$, room temperature). The supernatants were removed from the protein disks into a new Eppendorf tube, and the bottom layer (containing chloroform) was discarded. Then, the supernatants and protein disks were sonicated on ice twice for $3 \mathrm{~s}$. Trichloroacetic acid was added to the samples at a final concentration of $5 \%$ followed by incubation on ice for $10 \mathrm{~min}$. The precipitates were pelleted by centrifugation for $10 \mathrm{~min}$ (13,000 rpm, room temperature). The supernatants were discarded, and the pellets were redissolved in Tris buffer $(70 \mathrm{mM}$, $\mathrm{pH}$ 8.0). The sonication was repeated as described earlier.

Then, the lung, liver, and prepared brain samples were processed similarly. To each sample, $2 \times$ concentrated sodium dodecyl sulfate (SDS)-polyacrylamide gel electrophoresis sample buffer was added. Proteins were separated on $10 \%$ SDS-polyacrylamide gel and transferred to nitrocellulose membranes. After blocking ( $1 \mathrm{~h}$ with $2 \%$ non-fat milk in Tris-buffered saline), membranes were probed overnight at $4^{\circ} \mathrm{C}$ with primary antibodies $(1: 1,000)$ binding to the $75-\mathrm{kDa}$ COX-2 protein (catalog nr: ab15191; Abcam 
Plc., Cambridge, UK). Based on the previous studies measuring LPS-induced COX-2 protein expression with Western blot (30, 35,36 ), we used GAPDH as the loading reference. Membranes were washed six times for $5 \mathrm{~min}$ in Tris-buffered saline, containing $0.1 \%$ Tween before addition of goat anti-rabbit horseradish peroxidase-conjugated secondary antibody (1:3,000 dilution; Bio-Rad, Budapest, Hungary). Membranes were washed again as before, and the antibody-antigen complexes were visualized by enhanced chemiluminescence. After scanning, the results were quantified by using ImageJ software (NIH, Bethesda, MD, USA).

\section{Data Processing and Analysis}

Data on deep $T_{\mathrm{b}}$, tail $T_{\mathrm{sk}}$, and $\mathrm{VO}_{2}$ were compared by three-way ANOVA, while for comparison of serum cytokine levels, COX-2 expression, and $\mathrm{PGE}_{2}$ concentrations two-way ANOVA was used. As in our previous studies $(25,26)$, ANOVA was followed by the Fisher least significant difference post hoc test. For statistical analysis, Sigmaplot 11.0 (Systat Software, San Jose, CA, USA) software was used. The effects were considered significant when $P<0.05$. All data are reported as mean \pm SE.

\section{RESULTS}

\section{Thermoregulatory Phenotype of Tacr1 ${ }^{+/+}$ and Tacr1 $^{-/-}$Mice}

The basal deep $T_{\mathrm{b}}$ of the mice was nearly identical regardless of either gender or genotype in the thermocouple setup throughout the time period when substance administrations were performed in the fever experiments (Figure S1 in Supplementary Material).

To evaluate whether $\mathrm{Tacr} 1^{-/-}$mice can appropriately activate warmth- and cold-defense mechanisms, we studied the thermoregulatory response of these mice to ambient heating and cooling, respectively. To reveal even a small deficiency in heat defenses, we used a severe heat exposure model that results in $\sim 6^{\circ} \mathrm{C}$ rise in deep $T_{\mathrm{b}}$. When exposed to heat, the mice of both genotypes ( $\mathrm{TaCr}^{+/+}$and $\mathrm{TaCr} 1^{-/-}$) responded with rapid, nearmaximal tail skin vasodilation with $T_{\text {sk }}$ approaching $41^{\circ} \mathrm{C}$ (Figure $\mathrm{S} 2 \mathrm{~A}$ in Supplementary Material). Neither the $T_{\mathrm{sk}}$ response nor the $T_{\mathrm{b}}$ response differed between the genotypes. Hence, Tacr $1^{-/-}$mice are fully capable of increasing heat loss through their tails and defending their deep $T_{\mathrm{b}}$ against heat.

To reveal even a small deficiency in cold defenses, we used a severe cold exposure model that results in a pronounced drop in deep $T_{\mathrm{b}}$. When exposed to cold in this model, the mice of both genotypes responded with tail skin vasoconstriction (a decrease in $T_{\mathrm{sk}}$ ), but even so their $T_{\mathrm{b}}$ decreased by $\sim 6^{\circ} \mathrm{C}$ (Figure $\mathrm{S} 2 \mathrm{~B}$ in Supplementary Material). The response dynamics did not differ between the two genotypes. Hence, the thermoregulatory response of $\mathrm{Tacr}^{-1-}$ mice to cooling is unaltered.

\section{Characteristics of the Thermoregulatory Response of Tacr1 $^{+/+}$and Tacr1 ${ }^{-/-}$Mice to LPS}

In thermophysiological experiments, we compared the fever response between TacrI ${ }^{+/+}$and Tacr $1^{-/-}$mice. When treated with LPS $(120 \mu \mathrm{g} / \mathrm{kg}$, i.p.), the mice of both genotypes developed fever as compared to saline-treated mice (Figures 1A,B). However, the effects of both the treatment $\times$ time interaction [ANOVA, $\left.F_{(42,1376)}=2.114, P<0.001\right]$ and treatment $\times$ genotype interaction $\left[F_{(1,1376)}=40.908, P<0.001\right]$ were significant on their $T_{\mathrm{b}}$ response. LPS-treated $\mathrm{Tacr}^{+/+}$mice responded with a typical fever response: their deep $T_{\mathrm{b}}$ started to increase at $20 \mathrm{~min}$, plateaued $\left(\sim 38.5^{\circ} \mathrm{C}\right)$ between 40 and $100 \mathrm{~min}$, then it gradually decreased, but remained elevated compared to saline treatment throughout the experiment (Fisher LSD test, $P<0.05$ at 30-360 $\mathrm{min}$ ). These findings are in line with those reported previously (37). In $\mathrm{Tacr}^{-/-}$mice, the LPS-induced fever response was less pronounced than in $T a c r 1^{+/+}$mice, reaching the level of significance at 40-110 min compared to saline treatment (Fisher LSD test, $P<0.05$ ) (Figure 1A). The LPS-induced increase in deep $T_{\mathrm{b}}$ was brought about by an elevation of $\mathrm{VO}_{2}$, changing with parallel dynamics as $T_{\mathrm{b}}$ in both genotypes (Figures 1A,B). Similar to $T_{\mathrm{b}}$, the effects of both the treatment $\times$ time interaction [ANOVA, $F_{(42,1161)}=1.618, P<0.01$ ] and treatment $\times$ genotype interaction $\left[F_{(1,1161)}=15.802, P<0.001\right]$ were significant on the $\mathrm{VO}_{2}$ of the mice. After LPS treatment, the $\mathrm{VO}_{2}$ was significantly higher compared to saline at 40-350 min in $\mathrm{Tacr}^{+/+}$mice (Fisher LSD test, $P<0.05$ ) and at $40-140 \mathrm{~min}$ in Tacr $1^{-/-}$mice (Fisher LSD test, $P<0.05)$. Since the experiments were carried out at the lower end of the thermoneutral zone, the mice exhibited cutaneous vasoconstriction (as indicated by their low tail $T_{\mathrm{sk}}$ ), thus no further decrease in tail $T_{\mathrm{sk}}$ was observed. Importantly, the $T_{\mathrm{b}}$ of the LPS-treated $\mathrm{Tacr} 1^{-/-}$mice was markedly $\left(0.5-0.7^{\circ} \mathrm{C}\right)$ lower than that of $\mathrm{TaCr}^{+/+}$mice starting from $40 \mathrm{~min}$ post-LPS infusion until the end of the experiment. Parallel with $T_{\mathrm{b}}$, the LPS-induced elevation of $\mathrm{VO}_{2}$ was also suppressed in $\mathrm{Tacr} 1^{-/-}$mice compared to their $\mathrm{Tacr}^{+/+}$littermates (Figure 1A). The LPS-induced elevation of both parameters was significantly attenuated in Tacr $1^{-/-}$mice compared to Tacr $1^{+/+}$mice at 40-120 min (Fisher LSD test, $P<0.05$ for intergenotype difference) (Figure 1A). The infusion of saline did not cause any effect on deep $T_{\mathrm{b}}$, tail $T_{\mathrm{sk}}$, and $\mathrm{VO}_{2}$ in the mice of either genotype (Figure 1B). Our results demonstrate that LPS-induced fever is attenuated in $\mathrm{TaCr}^{-/-}$mice already in the early stage (i.e., starting from $\sim 40 \mathrm{~min}$ ). Next, we wanted to know the suppression of which part of the "pyrogenic cytokine-COX-2-PGE 2 axis" is responsible for attenuating the fever response in the absence of Tacr1.

\section{The Thermoregulatory Effects of $\mathrm{PGE}_{2}$ and SP in $\mathrm{TaCr1}^{+/+}$and Tacr1 ${ }^{-/-}$Mice}

First, we studied whether the effect of $\mathrm{PGE}_{2}$, i.e., the key mediator of fever (2), is also reduced in the Tacr $1^{-/-}$mice. As the main site of the febrigenic action of $\mathrm{PGE}_{2}$ is situated in the preoptic area of the hypothalamus (10), we compared the thermoregulatory effects of i.c.v. injected $\mathrm{PGE}_{2}$ between $\mathrm{Tacr}^{+/+}$and Tacr1 ${ }^{-/-}$ mice. In response to $\mathrm{PGE}_{2}$ the mice of both genotypes rapidly developed a marked elevation in deep $T_{\mathrm{b}}$ and $\mathrm{VO}_{2}$ (Figure 2A), while administration of the vehicle did not cause any effects in either genotype (Figure 2B). The effect of the treatment $\times$ time interaction was significant with regard to both $T_{\mathrm{b}}$ and $\mathrm{VO}_{2}$ [ANOVA, $F_{(18,380)}=29.406, P<0.001$ and $F_{(18,380)}=11.922$, $P<0.001$, respectively], whereas the effects of the genotype or the 
Tacr1 $^{-1-}$

○ $\mathrm{Tacr}^{+/+}$

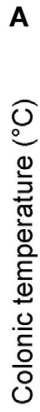

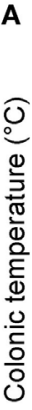

(8)

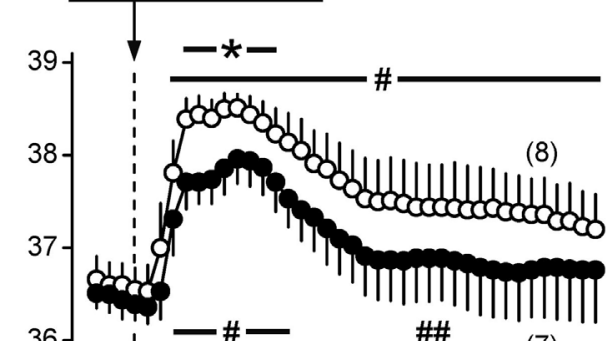

36-
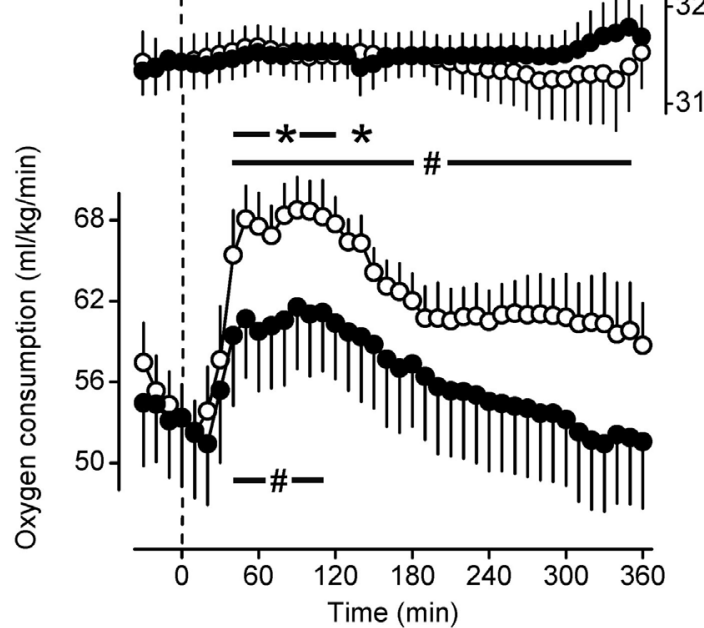
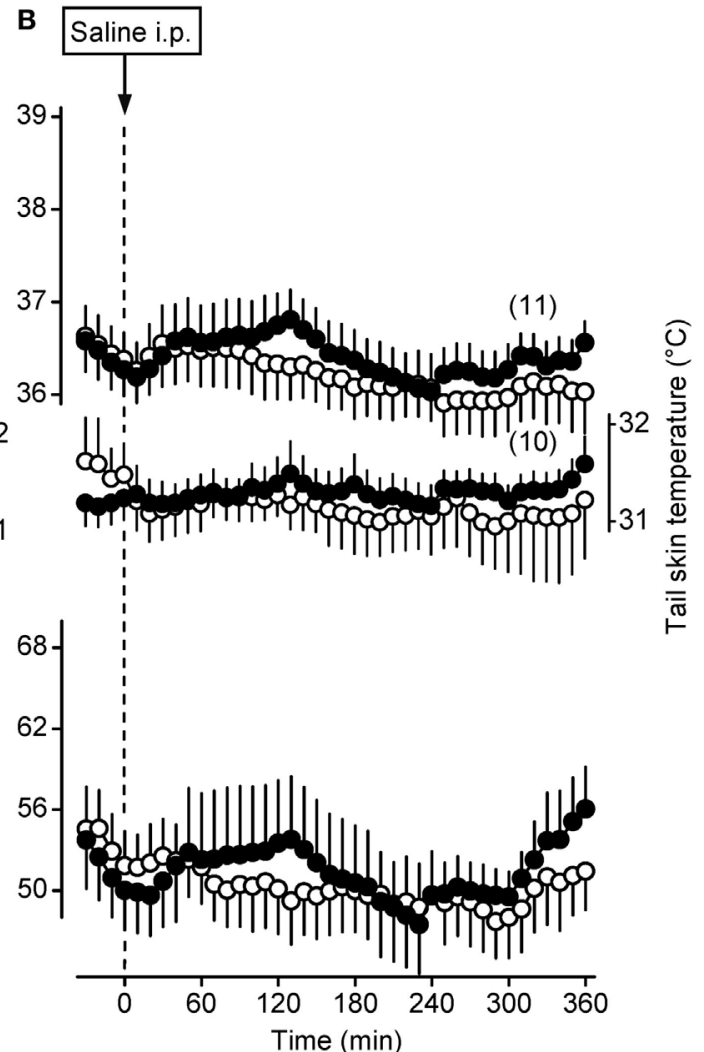

$\frac{0}{\frac{0}{2}}$

FIGURE 1 | Thermoeffector and colonic temperature responses of Tacr1 ${ }^{+/+}$and Tacr $^{-/-}$mice to lipopolysaccharide (LPS) (A) or saline (B) administered intraperitoneally (i.p.). The changes in colonic temperature [a form of deep body temperature ( $\left.T_{\mathrm{b}}\right)$ ] are shown in the upper panel; alterations in the activity of the two main autonomic thermoeffectors, skin temperature $\left(T_{\text {sk }}\right)$ and rate of oxygen consumption $\left(\mathrm{VO}_{2}\right)$ are depicted in the middle and lower panels, respectively. These experiments were performed in the respirometry setup at an ambient temperature of $31^{\circ} \mathrm{C}$. Number of animals in the corresponding groups are indicated in the figure. ${ }^{\star} P<0.05$, intergenotype difference in the response to LPS. ${ }^{*} P<0.05$, LPS vs. saline difference within the same genotype as determined by the Fisher LSD test.

treatment $\times$ genotype interaction were not significant on either deep $T_{\mathrm{b}}$ or $\mathrm{VO}_{2}$. Of note, there was no attenuation in either the $T_{\mathrm{b}}$ or $\mathrm{VO}_{2}$ rise in the Tacr1 $1^{-/-}$mice compared to their Tacr $1^{+/+}$ littermates. These experiments were conducted under identical conditions as those with LPS, including the ambient temperature $\left(31^{\circ} \mathrm{C}\right)$ near the lower end of the thermoneutral zone, which can explain why we did not observe any change in the tail $T_{\mathrm{sk}}$ in the mice of either genotype. Our results with $\mathrm{PGE}_{2}$ rule out the possibility that $\mathrm{TaCr}^{-/-}$mice are unable to activate their thermogenesis and increase their $T_{\mathrm{b}}$ to stimuli other than LPS. More importantly, the lack of attenuation in the $\mathrm{PGE}_{2}$-induced thermoregulatory response of the Tacr1 ${ }^{-/-}$mice suggests that their reduced fever response to LPS is due to a more upstream suppression in the febrigenic molecular pathway.

In a separate set of experiments, we confirmed the absence or presence of functional NK1 receptors in the Tacr $1^{-/-}$and Tacr $1^{+/+}$mice, respectively. It has been repeatedly shown that SP evokes an increase in deep $T_{\mathrm{b}}$ by acting on the NK1 receptor $(15,16)$, therefore, we injected SP $(100 \mu \mathrm{g} / \mathrm{kg}$, i.c.v. $)$ or its vehicle to $\mathrm{Tacr} 1^{+/+}$and $\mathrm{Tacr}^{-/-}$mice and compared their $T_{\mathrm{b}}$ responses
(Figures 2C,D). We found that the SP-induced increase in deep $T_{\mathrm{b}}$ was practically absent in $T a c r 1^{-/-}$mice, whereas it was significant in $\mathrm{Tacr} \mathrm{1}^{+/+}$mice at 20-60 min compared to vehicle treatment (Fisher LSD test, $P<0.05$ ) (Figures 2C,D). The effects of both the genotype [ANOVA, $F_{(1,260)}=10.538, P<0.01$ ] and the treatment $\times$ genotype interaction $\left[F_{(1,260)}=13.233\right.$, $P<0.001]$ were significant. In the SP-treated mice, there was a significant difference between the $T_{\mathrm{b}}$ of Tacr1 ${ }^{+/+}$and Tacr1 $1^{-/-}$ mice at 10-40 min (Fisher LSD test, $P<0.05$ ), which confirms the validity of this animal model.

\section{LPS-Induced Changes in Serum Cytokine Levels of $\mathrm{TaCr1}^{+/+}$and $\mathrm{TaCr1}^{-/-}$Mice}

One of the early steps in LPS-induced fever signaling is the activation of innate immune cells, including macrophages, leukocytes, and dendritic cells, which, in turn leads to augmented production of inflammatory cytokines, including TNF- $\alpha$ and IL-6 (3). Thus, in our next experiments we measured serum concentrations of TNF- $\alpha$ and IL-6 in the Tacr $1^{+/+}$and $\mathrm{Tacr}^{-/-}$mice to assess 


\section{Tacr1 ${ }^{-1-} \quad$ O Tacr1 ${ }^{+/+}$}
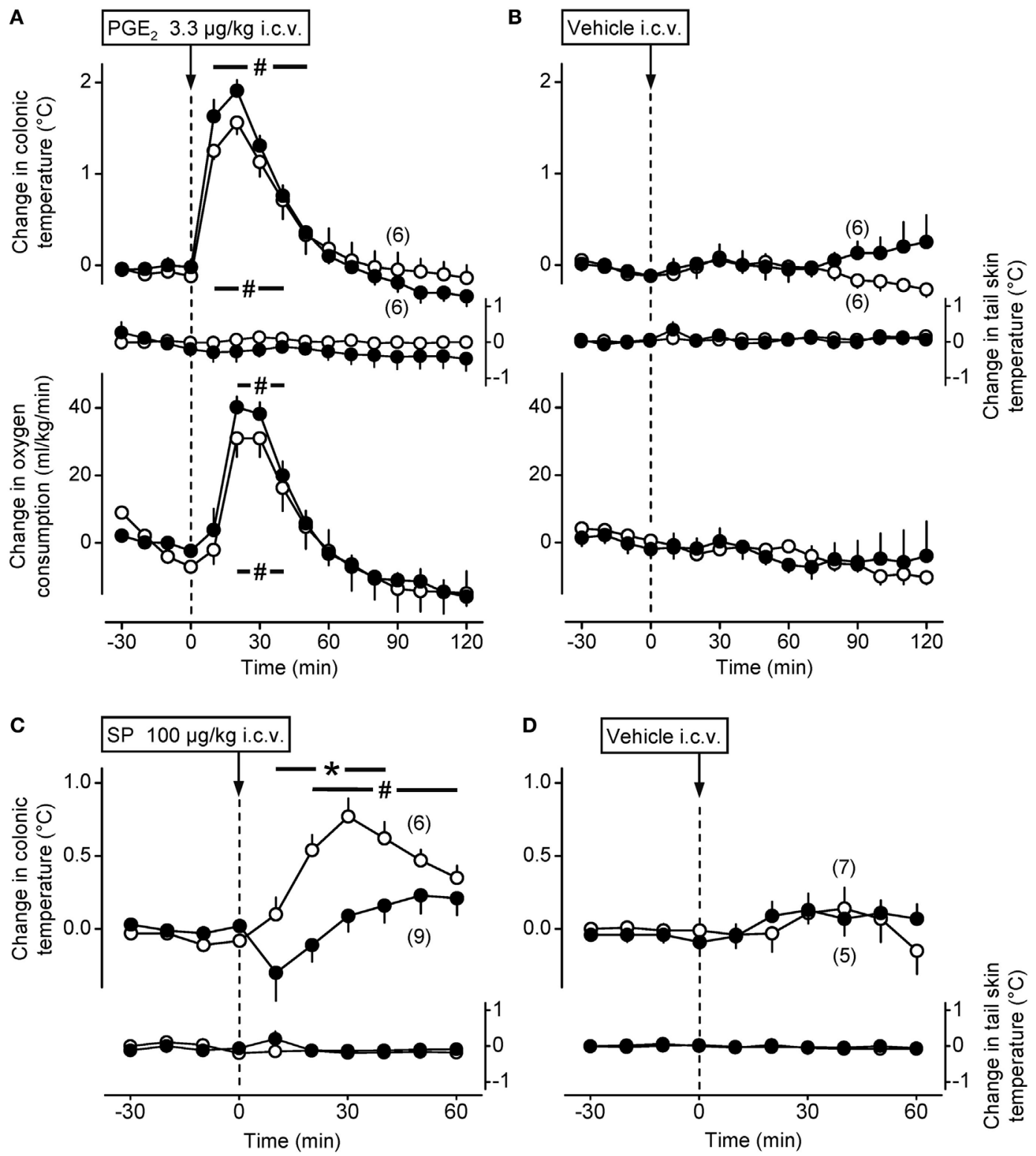

FIGURE 2 | The thermoregulatory response of Tacr1 $^{1 /+}$ and Tacr1 $1^{-/-}$mice to prostaglandin (PG) $\mathrm{E}_{2}$ and substance $\mathrm{P}(\mathrm{SP})$ administered intracerebroventricularly (i.c.V.). Changes in colonic temperature (upper panel), skin temperature $\left(T_{\text {sk }}\right.$ (middle panel), and rate of oxygen consumption $\left(\mathrm{VO}_{2}\right)$ (bottom panel) in response to $\mathrm{PGE}_{2}$ (dose indicated) (A) and its vehicle (B) in Tacr1 ${ }^{+/+}$and $\mathrm{TaCr}^{-/-}$mice. The experimental conditions were identical to those described in Figure 1 (respirometry setup, ambient temperature of $31^{\circ} \mathrm{C}$ ). At the time of the injection, the values of colonic temperature of the Tacr $1^{+/+}$and Tacr $1^{-/-}$mice were, respectively, $37.0 \pm 0.2$ and $37.1 \pm 0.1^{\circ} \mathrm{C}$ for $\mathrm{PGE}_{2}$-treated mice and $36.8 \pm 0.3$ and $36.9 \pm 0.2^{\circ} \mathrm{C}$ for vehicle-treated mice. These values did not differ statistically from each other. Changes in colonic temperature and $T_{\text {sk }}$ in response to SP (dose indicated) $(\mathbf{C})$ and its vehicle (D) in Tacr $1^{+/+}$and Tacr $^{-/-}$mice. These experiments were performed in the thermocouple setup at an ambient temperature of $33^{\circ} \mathrm{C}$. At the time of the injection, the values of colonic temperature of the Tacr $1^{1 /+}$ and Tacr $1^{-/-}$mice were, respectively, $37.2 \pm 0.2$ and $37.1 \pm 0.2^{\circ} \mathrm{C}$ for SP-treated mice and $37.3 \pm 0.3$ and $37.3 \pm 0.1^{\circ} \mathrm{C}$ for vehicle-treated mice. These values did not differ statistically from each other. Number of animals in the corresponding groups are indicated in the figure. ${ }^{*} P<0.05$, intergenotype difference in the response to $S P$. ${ }^{*} P<0.05$, treatment $\left(\mathrm{PGE}_{2}\right.$ or $\left.\mathrm{SP}\right)$ vs. vehicle difference within the same genotype as determined by the Fisher LSD test.

whether the LPS-induced cytokine production is suppressed in the absence of the NK1 receptor. The serum concentrations of TNF- $\alpha$ and IL- 6 did not differ between the genotypes after the infusion of saline (Figures $\mathbf{3 A}, \mathbf{B})$. The administration of LPS resulted in a substantial upsurge of TNF- $\alpha$ (Fisher LSD test, $P<0.001$ vs. saline for both genotypes) (Figure 3A) and IL-6 $(P<0.01$ for $\mathrm{Tacrl}^{+/+}$mice and $P<0.001$ for $\mathrm{Tacrl}^{-/-}$mice) (Figure 3B). Importantly, we did not detect any significant difference in the 


$$
\text { Tacr1 }^{-/-} \quad \square \text { Tacr1 }^{+/+}
$$

A

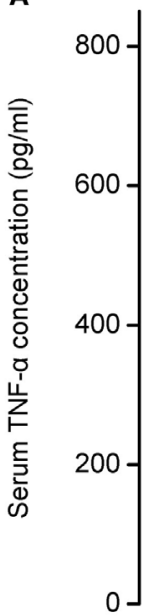

B

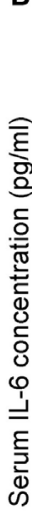

(10)

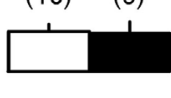

saline i.p.

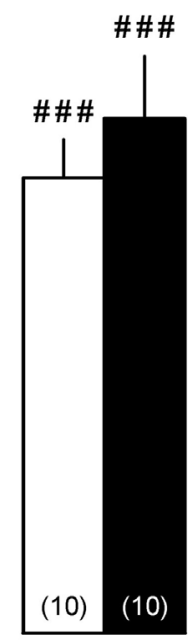

LPS $120 \mu \mathrm{g} / \mathrm{kg}$ i.p.

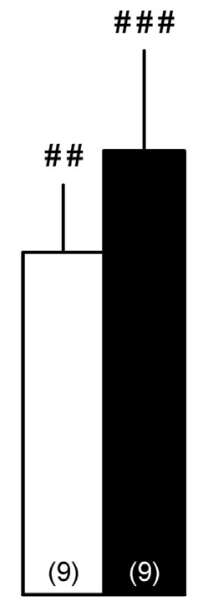

LPS $120 \mu \mathrm{g} / \mathrm{kg}$ i.p.

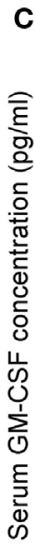

saline i.p.

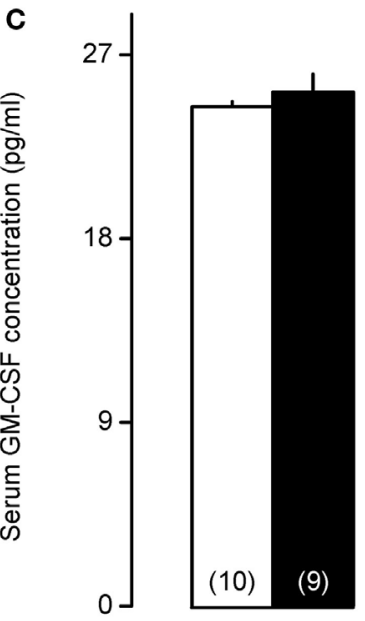

saline i.p.

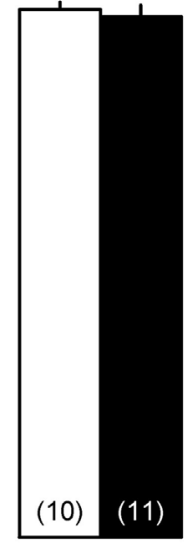

LPS $120 \mu \mathrm{g} / \mathrm{kg}$ i.p.

FIGURE 3 | Continued

FIGURE 3 Serum cytokine concentrations in Tacr1 ${ }^{+/+}$and Tacr1 $1^{-/-}$mice. (A) Serum tumor necrosis factor (TNF)- $\alpha$ concentrations in TaCr1 $1^{+/}$and $\mathrm{TaCr}^{-1-}$ mice in response to lipopolysaccharide (LPS) (dose indicated) or saline. (B) Serum IL-6 concentrations in TaCr1 ${ }^{+/+}$and Tacr1 ${ }^{-/-}$mice in response to LPS (dose indicated) or saline. (C) Serum granulocytemacrophage colony-stimulating factor (GM-CSF) concentrations in Tacr1 ${ }^{+/+}$ and $\mathrm{TaCr}^{-1-}$ mice in response to LPS (dose indicated) or saline. Blood samples were collected at 40 min postinfusion. Number of animals in the corresponding groups are indicated in the figure. Within each genotype, significant differences in the response to LPS (as compared to saline) are marked as ${ }^{\# P} P<0.01$ or ${ }^{\# \# P} P<0.001$ as determined by the Fisher LSD test.

TNF- $\alpha$ and IL- 6 concentrations between Tacr $1^{+/+}$and Tacr $1^{-/-}$ mice (Figures 3A,B).

It was shown that LPS can induce the secretion of GM-CSF in mice, which, in turn, can stimulate the production of inflammatory cytokines, such as TNF- $\alpha$ (38). We wanted to know whether changes in serum GM-CSF concentration can play a role in the attenuated fever response of the $\mathrm{Tacr}^{-/-}$mice to LPS. In our experimental model, we did not find any difference in the serum concentrations of GM-CSF between LPS-treated and saline-treated mice of either genotype (Figure 3C). The absence of a surge in GM-CSF concentration in response to LPS could be due to the early time point ( $40 \mathrm{~min}$ post-LPS infusion) chosen for blood collection in our experiments.

These data indicate that impaired pyrogenic cytokine production does not contribute to the attenuated fever response of Tacr $1^{-/-}$mice to LPS.

\section{LPS-Induced Changes in COX-2} Expression in $\mathrm{TaCr}^{+/+}$and $\mathrm{TaCr}^{-/-}$Mice Changes in COX-2 mRNA Expression in the Lungs, Liver, and Brain of $\mathrm{TaCr}^{+/+}$and $\mathrm{TaCr}^{-/-}$Mice

We moved downstream in the fever signaling pathway and compared the LPS-induced expression of COX-2 between Tacr $1^{+/+}$and Tacr $1^{-/-}$mice. At the mRNA level, COX-2 expression is upregulated in rats already in the early phase of the febrile response ( 30-40 min after LPS infusion) both in peripheral organs (lungs and liver) and to a lesser extent in the brain (31). In the present study, our experiments with LPS revealed that the fever response of $\mathrm{Tacr}^{-/-}$mice was attenuated already at $\sim 40 \mathrm{~min}$ post-LPS infusion (Figure 1A), and therefore, we collected lung, liver, and brain samples at this time point and studied the COX-2 mRNA expression in these tissues. As compared with the values of expression in saline-treated mice, the administration of LPS caused transcriptional upregulation of COX-2 mRNA in the lungs (Figure 4A), in the liver (Figure 4B), and in the brain (Figure 4C) of $\mathrm{Tacr}^{+/+}$and Tacr1 $1^{-/-}$mice (Fisher LSD test, $P<0.001$ vs. saline for all three tissues). There was no significant difference between the genotypes in any of the three tissue samples. The magnitude of the LPS-induced increase in the level of COX-2 expression was not the same in the three organs studied: $\sim 5$-fold in the lungs, 17-20-fold in the liver, and 2-fold in the brain (Figures 4A-C). These results are well in accordance with earlier findings on the dynamics of LPS-induced COX-2 mRNA expression (31), but cannot explain the attenuation of the febrile response in the Tacr1 ${ }^{-/-}$mice. 
$\mathrm{Tacr1}^{\text {/- }} \square \mathrm{TaCr}^{+/+}$

A

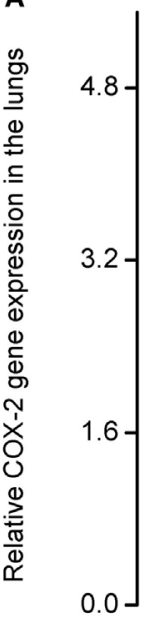

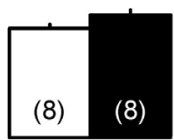

saline i.p.

LPS $120 \mu \mathrm{g} / \mathrm{kg}$ i.p.
(8) (8)

saline i.p
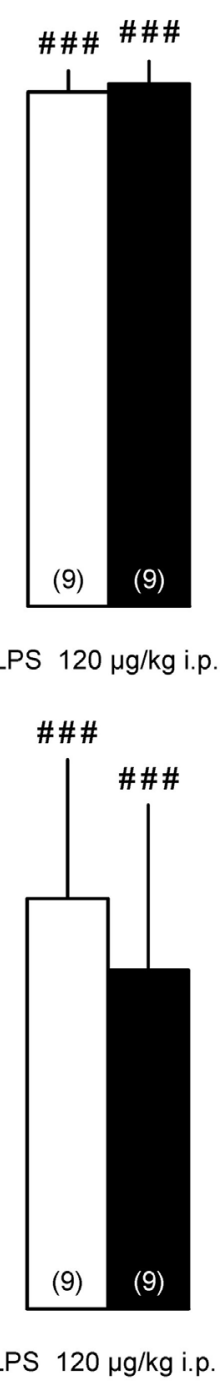

C

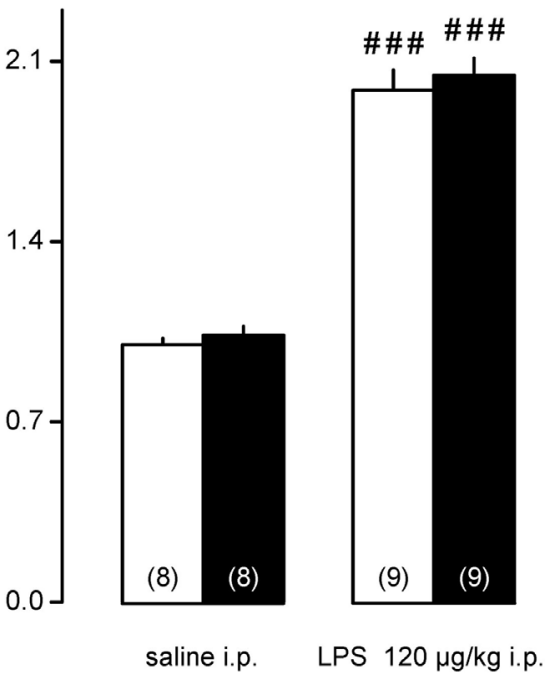

FIGURE 4 | Continued
FIGURE 4 | Relative cyclooxygenase-2 (COX-2) gene expression in the lungs (A), liver (B), and in the brain (C) of Tacr1 ${ }^{+/+}$and Tacr1 ${ }^{-/-}$mice after infusion of lipopolysaccharide (LPS) (dose indicated) or saline. Tissue samples were collected at $40 \mathrm{~min}$ postinfusion. Number of animals in the corresponding groups are indicated in the figure. Within each genotype, significant differences in the response to LPS (as compared to saline) are marked as ${ }^{\# \# P} P<0.001$ as determined by the Fisher LSD test.

Changes in COX-2 Protein Expression in the Lungs, Liver, and Brain of $\mathrm{TaCr}^{+/+}$and Tacr $1^{-/-}$Mice

Besides the LPS-induced amplified expression of COX-2 mRNA, the expression of the COX-2 protein was also found to increase in the periphery (but not in the brain) already in the early phase of the febrile response (9). Therefore, we also compared the COX-2 protein expression between LPS-treated $\mathrm{Tacr}^{+/+}$and $\mathrm{TaCr}^{-/-}$mice (Figure 5). In the lungs, the effects of both the treatment [ANOVA, $\left.F_{(1,26)}=6.165, P<0.05\right]$ and genotype $\left[F_{(1,26)}=8.532, P<0.01\right]$ were significant $($ Figure 5A). In the liver, the effect of treatment was also significant [ANOVA, $\left.F_{(1,28)}=6.555, P<0.05\right]$ (Figure 5B), whereas no significant change was found in the brain (Figure 5C). In $\mathrm{TaCrI}^{+/+}$mice, the administration of LPS resulted in a marked increase in COX-2 protein expression in the lungs (Fisher LSD test, $P<0.01$ vs. saline) and in the liver (Fisher LSD test, $P<0.05$ ), but not in the brain (Fisher LSD test, $P=0.264$ ). In contrast with the Tacr $1^{+/+}$ mice, the COX-2 protein expression did not change significantly in either the lungs or the liver of LPS-treated $\mathrm{TaCr}^{-/-}$mice as compared to saline treatment. In LPS-treated mice, the COX-2 protein expression was attenuated in the lungs of $\mathrm{TaCr}^{-/-}$mice compared with their $\mathrm{Tacr}^{+/+}$littermates (Fisher LSD test, $P<0.01$ ), while in the liver there was a tendency for reduced COX-2 expression in the LPS-treated $\mathrm{Tacrl}^{-/-}$mice (Fisher LSD test, $P=0.101$ ). These findings indicate that the absence of the NK1 receptor interferes with the augmentation of COX-2 expression at the protein level.

\section{Changes in $\mathrm{PGE}_{2}$ Concentration in the Lungs, Liver, and Brain of Tacr1 $^{+/+}$and Tacr1 $^{-/-}$Mice}

To assess whether the attenuated expression of COX-2 protein results in reduced production of $\mathrm{PGE}_{2}$ in the LPS-treated Tacr1 ${ }^{-/-}$ mice, we measured $\mathrm{PGE}_{2}$ concentrations in the lungs, liver, and brain of the mice. We found that the LPS treatment resulted in the biggest $(\sim 80 \%)$ increase in $\mathrm{PGE}_{2}$ concentration in the lungs (Figure 6A), followed by the liver ( $\sim 40 \%$ ) (Figure 6B), and then by the brain $(\sim 10 \%)$ (Figure $6 \mathrm{C})$. The effect of treatment was significant in the lungs [ANOVA, $F_{(1,15)}=7.065, P<0.05$ ], but not in the other two organs. Post hoc analysis revealed that the LPS-induced increase in $\mathrm{PGE}_{2}$ concentration was significant in the lungs of $\mathrm{Tacrl}^{+/+}$mice compared with saline (Fisher LSD test, $P<0.05)$, whereas in the lungs of Tacr1 ${ }^{-/-}$mice the $\mathrm{PGE}_{2}$ level did not increase significantly in response to LPS (Fisher LSD test, $P=0.275$ vs. saline). These results suggest that in the lungs of Tacr1 $1^{-/}$mice the LPS-induced surge in $\mathrm{PGE}_{2}$ concentration is reduced. 
A

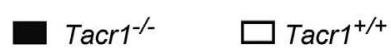

\begin{tabular}{|c|c|c|c|}
\hline $\mathrm{Tacr1}^{+/+}$ & Tacr1 $^{-1-}$ & $\mathrm{Tacr}^{+/+}$ & Tacr1 $^{1-}$ \\
\hline saline saline & saline saline & LPS & LPS \\
\hline
\end{tabular}
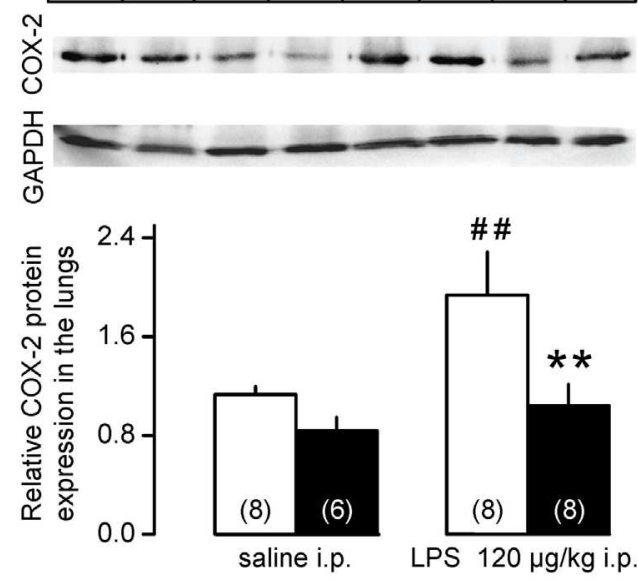

B

\begin{tabular}{|c|c|c|c|c|c|}
\hline $\mathrm{Tacr1}^{+/+}$ & Tacr1 $^{-/-}$ & $\mathrm{Tacr}$ & $1^{+/+}$ & Tac & $1^{-/-}$ \\
\hline saline saline & saline saline & LPS & LPS & LPS & \\
\hline
\end{tabular}

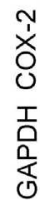

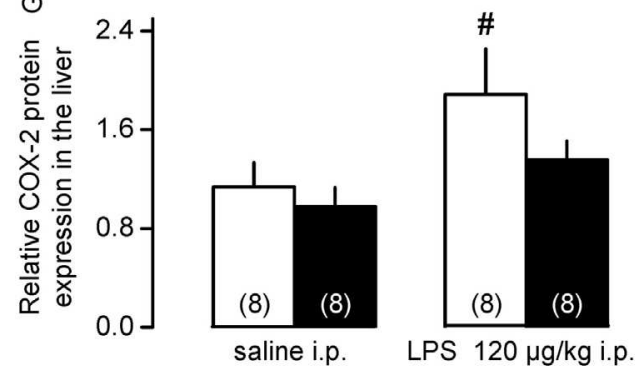

C

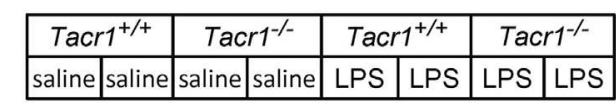

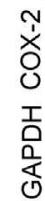
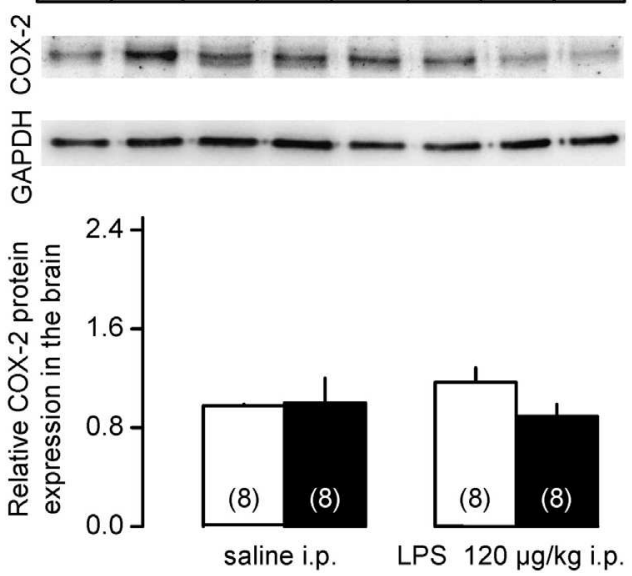

FIGURE 5 | Continued
FIGURE 5 | Relative cyclooxygenase-2 (COX-2) protein expression in the lungs (A), liver (B), and in the brain (C) of $\mathrm{TaCr}^{1+/}$ and $\mathrm{TaCr}^{-/-}$mice after infusion of lipopolysaccharide (LPS) (dose indicated) or saline. Tissue samples were collected at 40 min postinfusion. Number of animals in the corresponding groups are indicated in the figure. ${ }^{\star \star} P<0.01$, intergenotype difference in the response to LPS; ${ }^{*} P<0.05$ or ${ }^{\#} P<0.01$, LPS vs. saline difference within the same genotype as determined by the Fisher LSD test.

\section{DISCUSSION}

In our study, we showed that the absence of the NK1 receptor results in the attenuation of LPS-induced fever for the first time by using $\mathrm{Tacr}^{-/-}$mice. Our experimental model allowed us to detect the suppression of the febrile response already in the early phase of fever (starting from $\sim 40$ min post-LPS infusion), which is also a novel finding. When we looked at the molecular mechanism, we did not find a difference in the $\mathrm{PGE}_{2}$-induced febrile response between $\mathrm{Tacrl}^{+/+}$and $\mathrm{Tacrl}^{-/-}$mice. The LPS-induced serum cytokine production and COX-2 mRNA expression in the lungs, liver, and brain of the mice were also statistically indistinguishable between the genotypes. In contrast with mRNA, when we measured COX-2 expression at the protein level, we found that the LPS-induced surge was significantly attenuated in the lungs and tended to be suppressed in the liver of $\mathrm{Tacr}^{-/-}$mice as compared with their $\mathrm{Tacr} \mathrm{I}^{+/+}$littermates.

The involvement of SP signaling and the NK1 receptor in experimental fever was reported in earlier studies $(13-16,39)$. Antagonists of SP reduced the febrile response to LPS in rats and guinea pigs from the beginning of the response, which was detectable 45-90 min after LPS infusion $(13,14)$. However, when the authors looked at the mechanisms connecting the NK1 receptor with the fever signaling pathway, they focused on the later phases of fever (i.e., $2 \mathrm{~h}$ or more post-LPS infusion), presumably, because the early phase was absent in their experiments due to stress-hyperthermia as a consequence of stressful (needlepinch) drug injection (16). In the present study, we conducted the experiments under such conditions (extensive habituation, moderate LPS dose, non-stressful substance administration, and near neutral ambient temperature) that allowed us to study LPSinduced fever from 40 to 360 min postinfusion in mice, thus we could detect the attenuation of the response already at $40 \mathrm{~min}$ in the absence of Tacrl gene. A caveat in knockout mouse models is that compensatory mechanisms may develop. With regard to alteration of other NK receptors in mice genetically lacking the NK1 receptor, it was shown with RT-PCR and immunostaining that the expression of NK3 receptors was unchanged in the retina of $\mathrm{TacrI}^{-/-}$mice as compared with $\mathrm{Tacrl}^{+/+}$mice (40). However, in a mouse model of meningoencephalitis, the effects of combined treatment with NK2 and NK3 receptor antagonists were reduced on the neuroinflammatory scores in $\mathrm{TaCr}^{1^{--}}$mice compared with similarly treated $\mathrm{TacrI}^{+/+}$mice (41). It was concluded that in the genetic absence of NK1 receptors, tachykinins may utilize NK2 and NK3 receptors (41), although expression of the NK receptors was not measured. Taken together, in Tacr1 ${ }^{-/-}$ mice the expression of other NK receptors is presumably unchanged, but there might be an alteration in the utilization of NK2 and NK3 receptor-mediated mechanisms, which warrants 


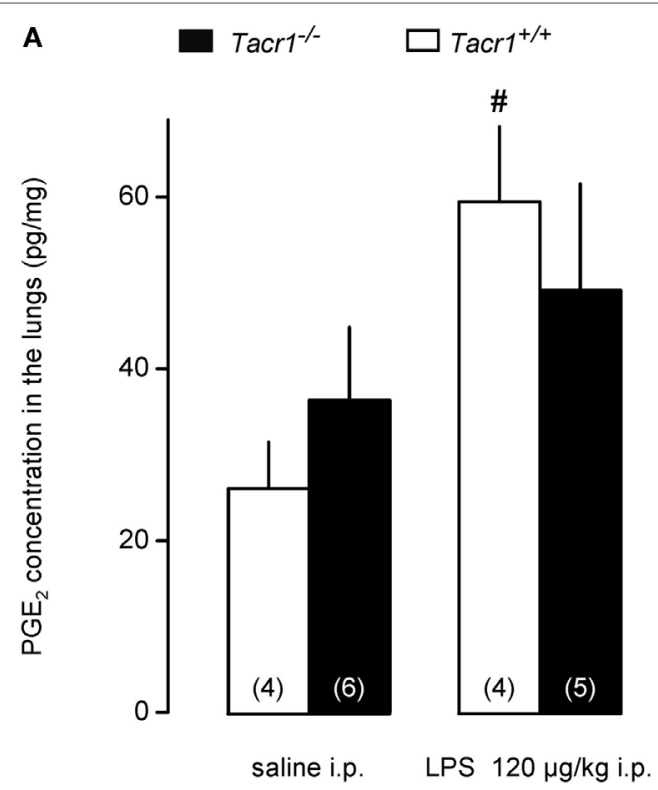

B

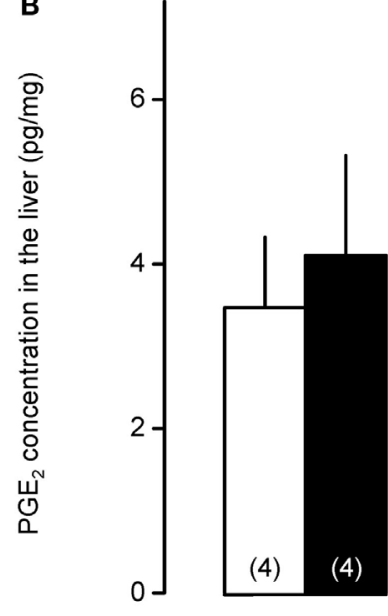

saline i.p.

(4)

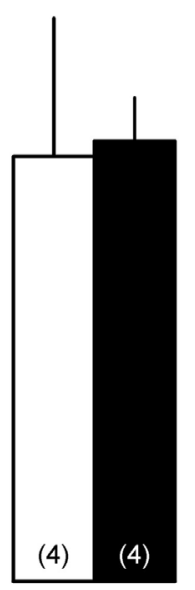

LPS $120 \mu g / k g$ i.p.

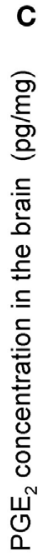
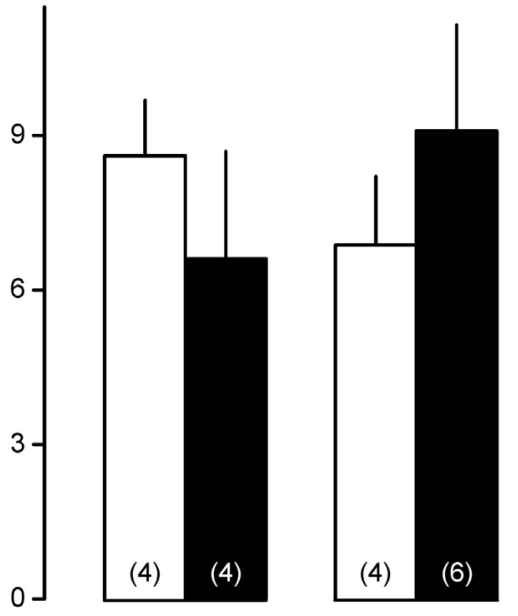

saline i.p.

LPS $120 \mu \mathrm{g} / \mathrm{kg}$ i.p.
FIGURE 6 | Prostaglandin (PG) $\mathrm{E}_{2}$ concentration in the lungs (A), liver (B), and in the brain (C) of Tacr1 ${ }^{+/+}$and Tacr1 $1^{-/-}$mice after infusion of lipopolysaccharide (LPS) (dose indicated) or saline. Tissue samples were collected at 40 min postinfusion. Number of animals in the corresponding groups are indicated in the figure. ${ }^{\#} P<0.05$, LPS vs. saline difference within the same genotype as determined by the Fisher LSD test.

for further characterization of the tachykinin pathways in this mouse model.

The later phases of fever are mediated mostly by increased $\mathrm{PGE}_{2}$ production in the brain, and it is well established that brainderived $\mathrm{PGE}_{2}$ is an important mediator for the maintenance of LPS-induced fever $(32,42-44)$. However, the early phase of fever is triggered from peripheral organs such as the lungs and the liver $(9,31)$. Therefore, our results suggest that the genetic blockade of the NK1 receptor interferes with fever signaling at a peripheral site of action in the early phase of LPS-induced fever. We further supported the peripheral action site of NK1 receptor in fever by showing that the $\mathrm{Tacr} 1^{-/-}$mice were equally able to increase their thermogenesis and deep $T_{\mathrm{b}}$ in response to i.c.v. administration of $\mathrm{PGE}_{2}$. We focused the second part of our study on exploring which step of fever signaling is altered in the Tacr $1^{-/-}$mice. In the periphery, SP signaling has been shown to play a role in the induction of pyrogenic cytokine production by macrophages (45) and in pulmonary macrophage activation (46). In acute lung injury after burns, SP was found to upregulate COX-2 activity (47). The expression of the NK1 receptor by macrophages is well documented (48-53), but it was also found in various other immune cells (1). With regard to leukocytes, neutrophil accumulation was significantly inhibited in $\mathrm{Tacr}^{-/-}$mice in lung injury induced by immune complexes (54) or acute pancreatitis (55). Attenuated leukocyte recruitment and lung injury were also observed with the NK1 receptor antagonist SR140333 in a murine model of polymicrobial sepsis induced by cecal ligation and puncture (56). In the same model, the authors later showed that the SP-induced pro-inflammatory response was mediated mainly by protein kinase $C$ (PKC)- $\alpha$ (57). Whether the NK1 receptor-mediated leukocyte recruitment occurs via a direct action on granulocytes or indirectly through other cell types (e.g., endothelial cells and bronchial epithelial cells) remains a question of debate [for a review, see Ref. (58)]. On the one hand, the expression of functional NK1 receptors was demonstrated on granulocytes in mice (59) and humans (60). In the latter study, it was also shown that the SP-induced COX-2 expression was mediated by NK1 receptors (60). On the other hand, different authors failed to detect the presence of NK1 receptors in human granulocytes $(52,61)$, which indicates that the NK1 receptormediated granulocyte migration develops via a primary effect of SP on other cell types. In line with such scenario, expression of the NK1 receptor was shown in several types of stromal cells in the lung, including bronchial glands, bronchial vessels, and bronchial smooth muscle (62), as well as airway epithelial cells $(63,64)$ and postcapillary venular endothelial cells $(65)$. With the help of nested PCR, the expression of the NK1 receptor was demonstrated also in the liver, predominantly in non-parenchymal cells, most likely macrophages, lymphocytes, and granulocytes, but also in hepatocytes (53), which contradicted earlier studies 
reporting no detectable expression of the NK1 receptor in the liver by using classical techniques (66-68). In lung epithelial cell cultures, SP via NK1 receptors stimulated neutrophil adherence (69) and pro-inflammatory cytokine production (70). Moreover, LPS enhanced the SP-induced neutrophil adherence and associated cytokine release via an involvement of NK1 receptors (71). Taken together, it is possible that LPS-induced leukocyte trafficking is reduced in $\mathrm{Tacr} 1^{-/-}$mice, which may be caused either by a direct effect of the NK1 receptor's absence on leukocytes or indirectly through other cell types such as lung epithelial cells. The reduced trafficking and therefore a reduced cellular infiltrate within the lung and the liver might contribute to reduced expression of COX-2.

As discussed earlier, many peripheral events of the fever response can be influenced by SP signaling. In the present study, we did not find difference in the serum levels of inflammatory cytokines (TNF- $\alpha$ and IL-6) between the LPS-treated Tacr $1^{+/+}$and Tacr $1^{-/-}$mice, which indicates that the activation of macrophages and their cytokine production is not impaired in the absence of the NK1 receptor. We did not measure the levels of the third major pro-inflammatory cytokine, IL-1 $\beta$, because it has been shown that it exerts its pyrogenic actions independently from the NK1 receptor (16). LPS can also modulate COX-2 transcriptionally and posttranscriptionally in macrophages independently from inflammatory cytokines (72). When we determined the COX-2 mRNA expression, we found that at this early time point ( $\sim 40 \mathrm{~min})$ it was greatly amplified in the lungs and liver, and to lesser extent in the brain of the mice, which results are in harmony with the previous findings (31). The lack of difference between the genotypes indicates that transcriptional upregulation of COX-2 is not influenced by the NK1 receptor. The correlation of mRNA and protein levels in biological samples is often poor (73), moreover, the expression of COX-2 is regulated not only at the level of transcription but also at the levels of post-transcription and translation $(72,74,75)$. Therefore, we also determined the expression of the COX-2 protein in the lungs, liver, and brain of the mice. In accordance with previous reports showing augmented expression of the COX-2 protein in the periphery (9), we also detected LPS-induced amplification of the COX-2 protein expression in the lungs and liver of $\mathrm{TaCr}^{+/+}$mice as compared with saline treatment, whereas we did not find significant increase in their brain. Importantly, however, the LPS-induced amplification of the expression of the COX-2 protein was attenuated in the lungs and tended to be suppressed in the liver of $\mathrm{Tacr}^{-/-}$mice as compared with their $\mathrm{Tacr}^{+/+}$littermates. In accordance with the different COX-2 protein expression between the genotypes, the administration of LPS caused a significant surge of $\mathrm{PGE}_{2}$ concentration in the lungs of $\mathrm{TaCr}^{+/+}$mice, which was absent in the Tacr1 $1^{-/-}$mice. It can be expected that the observed difference in pulmonary $\mathrm{PGE}_{2}$ synthesis also results in different plasma concentrations of $\mathrm{PGE}_{2}$ between the genotypes. The sensitive site where PGEs produce fever is located within the region of the brain that includes the organum vasculosum laminae terminalis and the surrounding preoptic area of the hypothalamus (76). Peripherally borne $\mathrm{PGE}_{2}$ can broadly penetrate in the perivascular space in the periventricular organs (such as the organum vasculosum laminae terminalis) and activate neurons or non-neural cells, thus trigger the febrile response (77). It has to be noted that fever signaling was not examined at later time points in the current experiments due to the study design, and therefore it cannot be excluded that COX-2 expression and $\mathrm{PGE}_{2}$ production in the brain are also affected by the blockade of the NK1 receptor, especially during the maintenance phase of fever. The experimental confirmation of the LPS-induced temporospatial distribution of $\mathrm{PGE}_{2}$ in the plasma, cerebrospinal fluid, and specific brain regions in the absence of the NK1 receptor remains subjects for future studies.

Our results demonstrate for the first time that at the onset of the fever response the NK1 receptor contributes to the augmentation of COX-2 protein expression in peripheral organs. In accordance with our findings, the modulation of COX-2 protein expression by SP signaling has been shown in several human cell lines, including polymorphonuclear leukocytes (60), colonic epithelial cells (78), and endothelial cells (79). Furthermore, an autocrine circuitry between $\mathrm{SP}$ and $\mathrm{PGE}_{2}$ production was recently suggested in the fever response to endogenous pyrogens (39). The exact mechanism, how SP signaling interacts with COX-2 expression remains to be elucidated in future studies. It is possible that the absence of the NK1 receptor leads to alterations in the expression of enzymes upstream (e.g., $\mathrm{PLA}_{2}$ ) or downstream of COX-2 (e.g., PGE synthases). An alteration in the phosphorylation (i.e., activation) of the cytosolic (c) form of $\mathrm{PLA}_{2}$ could be of particular interest, as in the fundamental study by Steiner et al. (9) LPS increased the contents of phosphorylated $\mathrm{CPLA}_{2}$ and COX-2 in the lung, but did not alter the protein level of constitutively expressed microsomal $\mathrm{PGE}_{2}$ synthase-1. In Chinese hamster ovary cells stably expressing NK1 receptors, SP induced the release of arachidonic acid, presumably by the activation of $\mathrm{CPLA}_{2}$, which was blocked by an antagonist of the NK1 receptor (80). Furthermore, in a mouse model of nerve injury, the enhanced activation of $\mathrm{CPLA}_{2}$ was abolished by NK1 receptor antagonist in neurons and possibly microglia in the spinal cord (81). These data indicate a link between activation of $\mathrm{CPLA}_{2}$ and $\mathrm{NK} 1$ receptormediated SP signaling at least in some cell types. Further studies are needed to assess whether the LPS-induced phosphorylation of $\mathrm{CPLA}_{2}$ is altered in the lungs of Tacr1 ${ }^{-/-}$mice in addition to the decreased COX-2 protein expression as shown in this study. A potential link in signal transduction between SP and COX-2 may coexist through PKC, as it was shown earlier that inhibitory effects of SP are mediated, at least in part by PKC isoenzymes (82), which play a key role in the biosynthesis of $\mathrm{PGE}_{2}$, likely by regulating the induction of COX-2 (83).

The findings of the present study further advance our understanding about the interactions between SP signaling and the "cytokine-COX-2-PGE " axis in experimental fever. As a perspective, our results can help to identify NK1 receptor as a drug target to suppress peripheral COX-2 activity.

\section{ETHICS STATEMENT}

All procedures were conducted under protocols approved by Institutional Animal Use and Care Committee of the University of Pecs and were in accordance with the directives of the National Ethical Council for Animal Research and those of the European Communities Council (86/609/EEC). 


\section{AUTHOR CONTRIBUTIONS}

Eszter P, PH, Erika P, and AG designed the study; Eszter P, VT, CZ, ZR, EO, PK, NK, RM, LD, KO, NS, KP, AK, and AG performed experiments; $\mathrm{PH}$ and Erika $\mathrm{P}$ provided compounds, mice, and analytic tools; Eszter P, VT, EO, RM, LD, KP, AK, and AG processed and analyzed data; and Eszter P, VT, LD, KP, AK, and AG wrote the paper. All the authors reviewed and finally approved the manuscript.

\section{ACKNOWLEDGMENTS}

The authors thank Drs. John Quinn and Andreas Zimmer for donating the $T a c r 1^{-/-}$mice. The authors are grateful to Aniko Varnagyne Rozsafi for the excellent technical assistance.

\section{REFERENCES}

1. Steinhoff MS, von Mentzer B, Geppetti P, Pothoulakis C, Bunnett NW. Tachykinins and their receptors: contributions to physiological control and the mechanisms of disease. Physiol Rev (2014) 94:265-301. doi:10.1152/ physrev.00031.2013

2. Ivanov AI, Romanovsky AA. Prostaglandin $\mathrm{E}_{2}$ as a mediator of fever: synthesis and catabolism. Front Biosci (2004) 9:1977-93. doi:10.2741/1383

3. Roth J, Blatteis CM. Mechanisms of fever production and lysis: lessons from experimental LPS fever. Compr Physiol (2014) 4:1563-604. doi:10.1002/ cphy.c130033

4. Dennis EA, Cao J, Hsu YH, Magrioti V, Kokotos G. Phospholipase $\mathrm{A}_{2}$ enzymes: physical structure, biological function, disease implication, chemical inhibition, and therapeutic intervention. Chem Rev (2011) 111:6130-85. doi: $10.1021 / \mathrm{cr} 200085 \mathrm{w}$

5. Nomura DK, Morrison BE, Blankman JL, Long JZ, Kinsey SG, Marcondes MC, et al. Endocannabinoid hydrolysis generates brain prostaglandins that promote neuroinflammation. Science (2011) 334:809-13. doi:10.1126/science. 1209200

6. Steiner AA, Hunter JC, Phipps SM, Nucci TB, Oliveira DL, Roberts JL, et al. Cyclooxygenase-1 or -2 - which one mediates lipopolysaccharide-induced hypothermia? Am J Physiol Regul Integr Comp Physiol (2009) 297:R485-94. doi:10.1152/ajpregu.91026.2008

7. Steiner AA, Rudaya AY, Robbins JR, Dragic AS, Langenbach R, Romanovsky AA. Expanding the febrigenic role of cyclooxygenase-2 to the previously overlooked responses. Am J Physiol Regul Integr Comp Physiol (2005) 289:R1253-7. doi:10.1152/ajpregu.00371.2005

8. Ueno N, Takegoshi Y, Kamei D, Kudo I, Murakami M. Coupling between cyclooxygenases and terminal prostanoid synthases. Biochem Biophys Res Commun (2005) 338:70-6. doi:10.1016/j.bbrc.2005.08.152

9. Steiner AA, Ivanov AI, Serrats J, Hosokawa H, Phayre AN, Robbins JR, et al. Cellular and molecular bases of the initiation of fever. PLoS Biol (2006) 4:e284. doi:10.1371/journal.pbio.0040284

10. Lazarus M, Yoshida K, Coppari R, Bass CE, Mochizuki T, Lowell BB, et al. EP3 prostaglandin receptors in the median preoptic nucleus are critical for fever responses. Nat Neurosci (2007) 10:1131-3. doi:10.1038/nn1949

11. Oka T, Oka K, Kobayashi T, Sugimoto Y, Ichikawa A, Ushikubi F, et al. Characteristics of thermoregulatory and febrile responses in mice deficient in prostaglandin EP1 and EP3 receptors. J Physiol (2003) 551:945-54. doi:10.1113/jphysiol.2003.048140

12. Zampronio AR, Soares DM, Souza GE. Central mediators involved in the febrile response: effects of antipyretic drugs. Temperature (Austin) (2015) 2:506-21. doi:10.1080/23328940.2015.1102802

13. Blatteis CM, Xin L, Quan N. Neuromodulation of fever. A possible role for substance P. Ann N Y Acad Sci (1994) 741:162-73. doi:10.1111/j.1749-6632. 1994.tb23097.x

14. Szelenyi Z, Szekely M, Balasko M. Role of substance P (SP) in the mediation of endotoxin (LPS) fever in rats. Ann N Y Acad Sci (1997) 813:316-23. doi:10.1111/j.1749-6632.1997.tb51713.x

\section{FUNDING}

This research has been supported by the National Research, Development and Innovation Office (grant FK 124483 to AG), the Medical School, University of Pecs (grant KA-2016-15 to AG), and the New National Excellence Program of the Hungarian Ministry of Human Capacities (grants UNKP-16-4-III and UNKP-17-4-III-PTE-33 to AG, UNKP-17-3-III-PTE-166 to KP, and UNKP-17-4-I-PTE-209 to LD).

\section{SUPPLEMENTARY MATERIAL}

The Supplementary Material for this article can be found online at http://www.frontiersin.org/articles/10.3389/fimmu.2018.00166/ full\#supplementary-material.

15. Balasko M, Szekely M, Szelenyi Z. The effect of CP-96,345, a non-peptide substance-P antagonist, on thermoregulation and the development of endotoxin-fever in rats. J Therm Biol (2000) 25:1-4. doi:10.1016/S03064565(99)00035-2

16. Reis RC, Brito HO, Fraga D, Cabrini DA, Zampronio AR. Central substance $\mathrm{P} \mathrm{NK}(1)$ receptors are involved in fever induced by LPS but not by IL-1beta and CCL3/MIP-1alpha in rats. Brain Res (2011) 1384:161-9. doi:10.1016/j. brainres.2011.02.001

17. Rupniak NM, Carlson EJ, Webb JK, Harrison T, Porsolt RD, Roux S, et al. Comparison of the phenotype of $\mathrm{NK}^{-/}$mice with pharmacological blockade of the substance $\mathrm{P}$ (NK1) receptor in assays for antidepressant and anxiolytic drugs. Behav Pharmacol (2001) 12:497-508. doi:10.1097/00008877200111000-00011

18. Kemeny LV, Hegyi P, Rakonczay Z Jr, Borka K, Korompay A, Gray MA, et al. Substance $\mathrm{P}$ inhibits pancreatic ductal bicarbonate secretion via neurokinin receptors 2 and 3 in the guinea pig exocrine pancreas. Pancreas (2011) 40:793-5. doi:10.1097/MPA.0b013e3182161d05

19. Guard S, Boyle SJ, Tang KW, Watling KJ, McKnight AT, Woodruff GN. The interaction of the NK1 receptor antagonist CP-96,345 with L-type calcium channels and its functional consequences. Br J Pharmacol (1993) 110:385-91. doi:10.1111/j.1476-5381.1993.tb13821.x

20. Rupniak NM, Carlson EJ, Shepheard S, Bentley G, Williams AR, Hill A, et al. Comparison of the functional blockade of rat substance $\mathrm{P}$ (NK1) receptors by GR205171, RP67580, SR140333 and NKP-608. Neuropharmacology (2003) 45:231-41. doi:10.1016/S0028-3908(03)00157-6

21. De Felipe C, Herrero JF, O'Brien JA, Palmer JA, Doyle CA, Smith AJ, et al. Altered nociception, analgesia and aggression in mice lacking the receptor for substance P. Nature (1998) 392:394-7. doi:10.1038/32904

22. Borbely E, Sandor K, Markovics A, Kemeny A, Pinter E, Szolcsanyi J, et al. Role of capsaicin-sensitive nerves and tachykinins in mast cell tryptaseinduced inflammation of murine knees. Inflamm Res (2016) 65:725-36. doi:10.1007/s00011-016-0954-x

23. Almeida MC, Hew-Butler T, Soriano RN, Rao S, Wang W, Wang J, et al. Pharmacological blockade of the cold receptor TRPM8 attenuates autonomic and behavioral cold defenses and decreases deep body temperature. J Neurosci (2012) 32:2086-99. doi:10.1523/JNEUROSCI.5606-11.2012

24. Garami A, Pakai E, McDonald HA, Reilly RM, Gomtsyan A, Corrigan JJ, et al. TRPV1 antagonists that cause hypothermia, instead of hyperthermia, in rodents: compounds' pharmacological profiles, in-vivo targets, thermoeffectors recruited, and implications for drug development. Acta Physiol (Oxf) (2018) (in press). doi:10.1111/apha.13038

25. Banki E, Pakai E, Gaszner B, Zsiboras C, Czett A, Bhuddi PR, et al. Characterization of the thermoregulatory response to pituitary adenylate cyclase-activating polypeptide in rodents. J Mol Neurosci (2014) 54:543-54. doi:10.1007/s12031-014-0361-0

26. Garami A, Pakai E, Oliveira DL, Steiner AA, Wanner SP, Almeida MC, et al. Thermoregulatory phenotype of the Trpvl knockout mouse: thermoeffector dysbalance with hyperkinesis. J Neurosci (2011) 31:1721-33. doi:10.1523/ JNEUROSCI.4671-10.2011 
27. de Oliveira C, Garami A, Lehto SG, Pakai E, Tekus V, Pohoczky K, et al. Transient receptor potential channel ankyrin-1 is not a cold sensor for autonomic thermoregulation in rodents. J Neurosci (2014) 34:4445-52. doi:10.1523/JNEUROSCI.5387-13.2014

28. Horvath A, Menghis A, Botz B, Borbely E, Kemeny A, Tekus V, et al. Analgesic and anti-inflammatory effects of the novel semicarbazide-sensitive amineoxidase inhibitor SzV-1287 in chronic arthritis models of the mouse. Sci Rep (2017) 7:39863. doi:10.1038/srep39863

29. Pohoczky K, Kun J, Szalontai B, Szoke E, Saghy E, Payrits M, et al. Estrogendependent up-regulation of TRPA1 and TRPV1 receptor proteins in the rat endometrium. J Mol Endocrinol (2016) 56:135-49. doi:10.1530/JME-15-0184

30. Engstrom Ruud L, Wilhelms DB, Eskilsson A, Vasilache AM, Elander L, Engblom D, et al. Acetaminophen reduces lipopolysaccharide-induced fever by inhibiting cyclooxygenase-2. Neuropharmacology (2013) 71:124-9. doi:10.1016/j.neuropharm.2013.03.012

31. Ivanov AI, Pero RS, Scheck AC, Romanovsky AA. Prostaglandin $E_{2}-$ synthesizing enzymes in fever: differential transcriptional regulation. Am J Physiol Regul Integr Comp Physiol (2002) 283:R1104-17. doi:10.1152/ ajpregu.00347.2002

32. Eskilsson A, Mirrasekhian E, Dufour S, Schwaninger M, Engblom D, Blomqvist A. Immune-induced fever is mediated by IL-6 receptors on brain endothelial cells coupled to STAT3-dependent induction of brain endothelial prostaglandin synthesis. J Neurosci (2014) 34:15957-61. doi:10.1523/ JNEUROSCI.3520-14.2014

33. Pfaffl MW. A new mathematical model for relative quantification in realtime RT-PCR. Nucleic Acids Res (2001) 29:e45. doi:10.1093/nar/29.9.e45

34. Riba A, Deres L, Eros K, Szabo A, Magyar K, Sumegi B, et al. Doxycycline protects against ROS-induced mitochondrial fragmentation and ISOinduced heart failure. PLoS One (2017) 12:e175195. doi:10.1371/journal. pone. 0175195

35. Kessen UA, Schaloske RH, Stephens DL, Killermann Lucas K, Dennis EA. $\mathrm{PGE}_{2}$ release is independent of upregulation of Group $\mathrm{V}$ phospholipase $\mathrm{A}_{2}$ during long-term stimulation of P388D1 cells with LPS. J Lipid Res (2005) 46:2488-96. doi:10.1194/jlr.M500325-JLR200

36. Steenport M, Khan KM, Du B, Barnhard SE, Dannenberg AJ, Falcone DJ. Matrix metalloproteinase (MMP)-1 and MMP-3 induce macrophage MMP-9: evidence for the role of TNF-alpha and cyclooxygenase-2. J Immunol (2009) 183:8119-27. doi:10.4049/jimmunol.0901925

37. Rudaya AY, Steiner AA, Robbins JR, Dragic AS, Romanovsky AA. Thermoregulatory responses to lipopolysaccharide in the mouse: dependence on the dose and ambient temperature. Am J Physiol Regul Integr Comp Physiol (2005) 289:R1244-52. doi:10.1152/ajpregu.00370.2005

38. Tiegs G, Barsig J, Matiba B, Uhlig S, Wendel A. Potentiation by granulocyte macrophage colony-stimulating factor of lipopolysaccharide toxicity in mice. J Clin Invest (1994) 93:2616-22. doi:10.1172/JCI117274

39. Brito HO, Barbosa FL, Reis RC, Fraga D, Borges BS, Franco CR, et al. Evidence of substance $\mathrm{P}$ autocrine circuitry that involves TNF-alpha, IL-6, and $\mathrm{PGE}_{2}$ in endogenous pyrogen-induced fever. J Neuroimmunol (2016) 293:1-7. doi:10.1016/j.jneuroim.2016.01.016

40. Catalani E, Dal Monte M, Gangitano C, Lucattelli M, Fineschi S, Bosco L, et al. Expression of substance P, neurokinin 1 receptors (NK1) and neurokinin 3 receptors in the developing mouse retina and in the retina of NK1 knockout mice. Neuroscience (2006) 138:487-99. doi:10.1016/j. neuroscience.2005.11.020

41. Kennedy PG, Rodgers J, Bradley B, Hunt SP, Gettinby G, Leeman SE, et al. Clinical and neuroinflammatory responses to meningoencephalitis in substance P receptor knockout mice. Brain (2003) 126:1683-90. doi:10.1093/ brain/awg160

42. Engstrom L, Ruud J, Eskilsson A, Larsson A, Mackerlova L, Kugelberg U, et al. Lipopolysaccharide-induced fever depends on prostaglandin $\mathrm{E}_{2}$ production specifically in brain endothelial cells. Endocrinology (2012) 153:4849-61. doi:10.1210/en.2012-1375

43. Rummel C, Matsumura K, Luheshi GN. Circulating IL-6 contributes to peripheral LPS-induced mPGES-1 expression in the rat brain. Brain Res Bull (2011) 86:319-25. doi:10.1016/j.brainresbull.2011.09.006

44. Rummel C, Sachot C, Poole S, Luheshi GN. Circulating interleukin-6 induces fever through a STAT3-linked activation of COX-2 in the brain. Am J Physiol Regul Integr Comp Physiol (2006) 291:R1316-26. doi:10.1152/ ajpregu.00301.2006
45. Lotz M, Vaughan JH, Carson DA. Effect of neuropeptides on production of inflammatory cytokines by human monocytes. Science (1988) 241:1218-21. doi:10.1126/science. 2457950

46. Boichot E, Germain N, Emonds-Alt X, Advenier C, Lagente V. Effects of SR 140333 and SR 48968 on antigen and substance P-induced activation of guinea-pig alveolar macrophages. Clin Exp Allergy (1998) 28:1299-305. doi:10.1046/j.1365-2222.1998.00398.x

47. Sio SW, Ang SF, Lu J, Moochhala S, Bhatia M. Substance P upregulates cyclooxygenase- 2 and prostaglandin E metabolite by activating ERK1/2 and NF-kappaB in a mouse model of burn-induced remote acute lung injury. J Immunol (2010) 185:6265-76. doi:10.4049/jimmunol.1001739

48. Ho WZ, Lai JP, Zhu XH, Uvaydova M, Douglas SD. Human monocytes and macrophages express substance $\mathrm{P}$ and neurokinin-1 receptor. JImmunol (1997) 159:5654-60.

49. Lai JP, Douglas SD, Ho WZ. Human lymphocytes express substance P and its receptor. J Neuroimmunol (1998) 86:80-6. doi:10.1016/S0165-5728(98)00025-3

50. Bost KL, Breeding SA, Pascual DW. Modulation of the mRNAs encoding substance P and its receptor in rat macrophages by LPS. Reg Immunol (1992) 4:105-12.

51. Marriott I, Bost KL. IL-4 and IFN-gamma up-regulate substance P receptor expression in murine peritoneal macrophages. J Immunol (2000) 165:182-91. doi:10.4049/jimmunol.165.1.182

52. Klassert TE, Pinto F, Hernandez M, Candenas ML, Hernandez MC, Abreu J, et al. Differential expression of neurokinin B and hemokinin-1 in human immune cells. J Neuroimmunol (2008) 196:27-34. doi:10.1016/j. jneuroim.2008.02.010

53. Bang R, Sass G, Kiemer AK, Vollmar AM, Neuhuber WL, Tiegs G. Neurokinin-1 receptor antagonists CP-96,345 and L-733,060 protect mice from cytokinemediated liver injury. J Pharmacol Exp Ther (2003) 305:31-9. doi:10.1124/ jpet.102.043539

54. Bozic CR, Lu B, Hopken UE, Gerard C, Gerard NP. Neurogenic amplification of immune complex inflammation. Science (1996) 273:1722-5. doi:10.1126/ science.273.5282.1722

55. Bhatia M, Saluja AK, Hofbauer B, Frossard JL, Lee HS, Castagliuolo I, et al. Role of substance $\mathrm{P}$ and the neurokinin 1 receptor in acute pancreatitis and pancreatitis-associated lung injury. Proc Natl Acad Sci U S A (1998) 95:4760-5. doi:10.1073/pnas.95.8.4760

56. Hegde A, Zhang H, Moochhala SM, Bhatia M. Neurokinin-1 receptor antagonist treatment protects mice against lung injury in polymicrobial sepsis. J Leukoc Biol (2007) 82:678-85. doi:10.1189/jlb.0407217

57. Hegde A, Koh YH, Moochhala SM, Bhatia M. Neurokinin-1 receptor antagonist treatment in polymicrobial sepsis: molecular insights. Int J Inflam (2010) 2010:601098. doi:10.4061/2010/601098

58. Maggi CA. The effects of tachykinins on inflammatory and immune cells. Regul Pept (1997) 70:75-90. doi:10.1016/S0167-0115(97)00029-3

59. Kaltreider HB, Ichikawa S, Byrd PK, Ingram DA, Kishiyama JL, Sreedharan SP, et al. Upregulation of neuropeptides and neuropeptide receptors in a murine model of immune inflammation in lung parenchyma. Am J Respir Cell Mol Biol (1997) 16:133-44. doi:10.1165/ajrcmb.16.2.9032120

60. Gallicchio M, Benetti E, Rosa AC, Fantozzi R. Tachykinin receptor modulation of cyclooxygenase-2 expression in human polymorphonuclear leucocytes. Br J Pharmacol (2009) 156:486-96. doi:10.1111/j.1476-5381.2008.00033.x

61. Marazziti D, Giannaccini G, Baroni S, Betti L, Giusti L, Lucacchini A, et al. Absence of NK1 receptors in human blood lymphocytes and granulocytes. Neuropsychobiology (2004) 50:221-5. doi:10.1159/000079974

62. Mapp CE, Miotto D, Braccioni F, Saetta M, Turato G, Maestrelli P, et al. The distribution of neurokinin-1 and neurokinin-2 receptors in human central airways. Am J Respir Crit Care Med (2000) 161:207-15. doi:10.1164/ ajrccm.161.1.9903137

63. Adcock IM, Peters M, Gelder C, Shirasaki H, Brown CR, Barnes PJ. Increased tachykinin receptor gene expression in asthmatic lung and its modulation by steroids. J Mol Endocrinol (1993) 11:1-7. doi:10.1677/jme.0.0110001

64. Chu HW, Kraft M, Krause JE, Rex MD, Martin RJ. Substance P and its receptor neurokinin 1 expression in asthmatic airways. J Allergy Clin Immunol (2000) 106:713-22. doi:10.1067/mai.2000.109829

65. Bowden JJ, Garland AM, Baluk P, Lefevre P, Grady EF, Vigna SR, et al. Direct observation of substance P-induced internalization of neurokinin 1 (NK1) receptors at sites of inflammation. Proc Natl Acad Sci U S A (1994) 91:8964-8. doi:10.1073/pnas.91.19.8964 
66. Cook GA, Elliott D, Metwali A, Blum AM, Sandor M, Lynch R, et al. Molecular evidence that granuloma T lymphocytes in murine Schistosomiasis mansoni express an authentic substance P (NK-1) receptor. J Immunol (1994) 152:1830-5.

67. Hershey AD, Krause JE. Molecular characterization of a functional cDNA encoding the rat substance P receptor. Science (1990) 247:958-62. doi:10.1126/ science. 2154852

68. Tsuchida K, Shigemoto R, Yokota Y, Nakanishi S. Tissue distribution and quantitation of the mRNAs for three rat tachykinin receptors. Eur J Biochem (1990) 193:751-7. doi:10.1111/j.1432-1033.1990.tb19396.x

69. DeRose V, Robbins RA, Snider RM, Spurzem JR, Thiele GM, Rennard SI, et al. Substance $\mathrm{P}$ increases neutrophil adhesion to bronchial epithelial cells. J Immunol (1994) 152:1339-46.

70. Williams R, Zou X, Hoyle GW. Tachykinin-1 receptor stimulates proinflammatory gene expression in lung epithelial cells through activation of NF-kappaB via a G(q)-dependent pathway. Am J Physiol Lung Cell Mol Physiol (2007) 292:L430-7. doi:10.1152/ajplung.00475.2005

71. Kuo HP, Lin HC, Hwang KH, Wang CH, Lu LC. Lipopolysaccharide enhances substance P-mediated neutrophil adherence to epithelial cells and cytokine release. Am J Respir Crit Care Med (2000) 162:1891-7. doi:10.1164/ ajrccm.162.5.9911065

72. Barrios-Rodiles M, Tiraloche G, Chadee K. Lipopolysaccharide modulates cyclooxygenase-2 transcriptionally and posttranscriptionally in human macrophages independently from endogenous IL-1 beta and TNF-alpha. J Immunol (1999) 163:963-9.

73. Maier T, Guell M, Serrano L. Correlation of mRNA and protein in complex biological samples. FEBS Lett (2009) 583:3966-73. doi:10.1016/j. febslet.2009.10.036

74. Park YK, Hong H, Jang BC. Transcriptional and translational regulation of COX-2 expression by cadmium in C6 glioma cells. Int J Mol Med (2012) 30:960-6. doi:10.3892/ijmm.2012.1052

75. Ristimaki A, Garfinkel S, Wessendorf J, Maciag T, Hla T. Induction of cyclooxygenase- 2 by interleukin-1 alpha. Evidence for post-transcriptional regulation. J Biol Chem (1994) 269:11769-75.

76. Oka T. Prostaglandin $\mathrm{E}_{2}$ as a mediator of fever: the role of prostaglandin E (EP) receptors. Front Biosci (2004) 9:3046-57. doi:10.2741/1458

77. Romanovsky AA, Ivanov AI, Karman EK. Blood-borne, albumin-bound prostaglandin $\mathrm{E}_{2}$ may be involved in fever. Am J Physiol (1999) 276:R1840-4.
78. Koon HW, Zhao D, Zhan Y, Rhee SH, Moyer MP, Pothoulakis C. Substance $\mathrm{P}$ stimulates cyclooxygenase-2 and prostaglandin $\mathrm{E}_{2}$ expression through JAK-STAT activation in human colonic epithelial cells. J Immunol (2006) 176:5050-9. doi:10.4049/jimmunol.176.8.5050

79. Gallicchio M, Rosa AC, Benetti E, Collino M, Dianzani C, Fantozzi R. Substance P-induced cyclooxygenase-2 expression in human umbilical vein endothelial cells. Br J Pharmacol (2006) 147:681-9. doi:10.1038/sj.bjp. 0706660

80. Garcia M, Sakamoto K, Shigekawa M, Nakanishi S, Ito S. Multiple mechanisms of arachidonic acid release in Chinese hamster ovary cells transfected with cDNA of substance P receptor. Biochem Pharmacol (1994) 48:1735-41. doi:10.1016/0006-2952(94)90459-6

81. Ma L, Nagai J, Chun J, Ueda H. An LPA species (18:1 LPA) plays key roles in the self-amplification of spinal LPA production in the peripheral neuropathic pain model. Mol Pain (2013) 9:29. doi:10.1186/1744-8069-9-29

82. Hegyi P, Rakonczay Z Jr, Tiszlavicz L, Varro A, Toth A, Racz G, et al. Protein kinase $\mathrm{C}$ mediates the inhibitory effect of substance $\mathrm{P}$ on $\mathrm{HCO}^{-}$secretion from guinea pig pancreatic ducts. Am J Physiol Cell Physiol (2005) 288:C1030-41. doi:10.1152/ajpcell.00430.2003

83. Molina-Holgado E, Ortiz S, Molina-Holgado F, Guaza C. Induction of COX-2 and $\mathrm{PGE}_{2}$ biosynthesis by IL-1beta is mediated by $\mathrm{PKC}$ and mitogenactivated protein kinases in murine astrocytes. Br J Pharmacol (2000) 131:152-9. doi:10.1038/sj.bjp.0703557

Conflict of Interest Statement: The authors declare that the research was conducted in the absence of any commercial or financial relationships that could be construed as a potential conflict of interest.

The reviewer DC and handling editor declared their shared affiliation.

Copyright (c) 2018 Pakai, Tekus, Zsiboras, Rumbus, Olah, Keringer, Khidhir, Matics, Deres, Ordog, Szentes, Pohoczky, Kemeny, Hegyi, Pinter and Garami. This is an open-access article distributed under the terms of the Creative Commons Attribution License (CC BY). The use, distribution or reproduction in other forums is permitted, provided the original author(s) and the copyright owner are credited and that the original publication in this journal is cited, in accordance with accepted academic practice. No use, distribution or reproduction is permitted which does not comply with these terms. 
II. 


\title{
Hyperbilirubinemia exaggerates endotoxin-induced hypothermia
}

\author{
Eszter Pakai ${ }^{1,2}$, Andras Garami ${ }^{1,2}$, Tatiane B Nucci ${ }^{1}$, Andrei I Ivanov ${ }^{1,3,4}$, and Andrej A Romanovsky ${ }^{1,3, *}$
}

\begin{abstract}
${ }^{1}$ FeverLab; Trauma Research; St. Joseph's Hospital and Medical Center; Phoenix, AZ USA; ${ }^{2}$ Department of Pathophysiology and Gerontology; Medical School; University of Pecs; Pecs, Hungary; ${ }^{3}$ Thermoregulation Laboratory; Legacy Holladay Park Clinical Research and Technology Center; Portland, OR USA; ${ }^{4}$ Department of Human and Molecular Genetics; Institute of Molecular Medicine; and Massey Cancer Center; Virginia Commonwealth University School of Medicine; Richmond, VA USA
\end{abstract}

Keywords: antioxidants, bilirubin, fever, Gunn rats, hepatic damage, lipopolysaccharides, LPS, liver, reactive oxygen species, ROS, transferases

Abbreviations: ALT, alanine aminotransferase; AST, aspartate aminotransferase; BUN, blood urea nitrogen; COX, cyclooxygenase; GGT, gamma-glutamyl transferase; LPS, lipopolysaccharide; NO, nitric oxide; PG, prostaglandin; ROS, reactive oxygen species; $T_{a}$, ambient temperature; $T_{b}$, body temperature.

Systemic inflammation is accompanied by an increased production of reactive oxygen species (ROS) and by either fever or hypothermia (or both). To study aseptic systemic inflammation, it is often induced in rats by the intravenous administration of bacterial lipopolysaccharide (LPS). Knowing that bilirubin is a potent ROS scavenger, we compared responses to LPS between normobilirubinemic Gunn rats (heterozygous, asymptomatic; J/+) and hyperbilirubinemic Gunn rats (homozygous, jaundiced; $J / J$ ) to establish whether ROS mediate fever and hypothermia in aseptic systemic inflammation. These two genotypes correspond to undisturbed versus drastically suppressed (by bilirubin) tissue accumulation of ROS, respectively. A low dose of LPS $(10 \mu \mathrm{g} / \mathrm{kg})$ caused a typical triphasic fever in both genotypes, without any intergenotype differences. A high dose of LPS $(1,000 \mu \mathrm{g} / \mathrm{kg})$ caused a complex response consisting of early hypothermia followed by late fever. The hypothermic response was markedly exaggerated, whereas the subsequent fever response was strongly attenuated in $\mathrm{J} / \mathrm{J}$ rats, as compared to $\mathrm{J} /+$ rats. $\mathrm{J} / \mathrm{J}$ rats also tended to respond to $1,000 \mu \mathrm{g} /$ $\mathrm{kg}$ with blunted surges in plasma levels of all hepatic enzymes studied (alanine aminotransferase, aspartate aminotransferase, gamma-glutamyl transferase), thus suggesting an attenuation of hepatic damage. We propose that the reported exaggeration of LPS-induced hypothermia in $\mathrm{J} / \mathrm{J}$ rats occurs via direct inhibition of nonshivering thermogenesis by bilirubin and possibly via a direct vasodilatatory action of bilirubin in the skin. This hypothermiaexaggerating effect might be responsible, at least in part, for the observed tendency of $\mathrm{J} / \mathrm{J}$ rats to be protected from LPS-induced hepatic damage. The attenuation of the fever response to $1,000 \mu \mathrm{g} / \mathrm{kg}$ could be due to either direct actions of bilirubin on thermoeffectors or the ROS-scavenging action of bilirubin. However, the experiments with $10 \mu \mathrm{g} / \mathrm{kg}$ strongly suggest that ROS signaling is not involved in the fever response to low doses of LPS.

\section{Introduction}

Fever and hypothermia are symptoms of infection and inflammation. They are triggered via the production and release of endogenous proinflammatory mediators, including prostaglandin (PG) $\mathrm{E}_{2}$, by pathogen-sensing immune cells in peripheral tissues and in the brain. ${ }^{1-5}$ Intracellular signaling cascades activated by these endogenous mediators are complex and involve lipid, peptide, gaseous, and other messengers (for review, see reference 6).

They also include the reactive oxygen species (ROS), such as hydrogen peroxide, superoxide anion, and nitric oxide (NO) ${ }^{7,8}$ ROS are unstable, short-lived molecules that are thought to have
2 major functions. First, they are downstream molecular effectors of immune cell activation and, as such, are directly involved in killing and clearing microbial pathogens. ${ }^{9}$ Second, they are messengers within several intracellular signaling pathways and, in this role, affect multiple cell functions. ${ }^{10}$ Signaling properties of ROS are based on their ability to interact with many intracellular proteins, including redox-sensitive transcriptional factors and ion channels, as well as metal-containing enzymes responsible for production of proinflammatory mediators (for review, see reference 11).

While production of ROS by different cells and tissues in response to bacterial lipopolysaccharide (LPS; often referred to as endotoxin) is well documented (for review, see reference 8), it is

(c) Eszter Pakai, Andras Garami, Tatiane B Nucci, Andrei I Ivanov, and Andrej A Romanovsky

*Correspondence to: Andrej A Romanovsky; Email: andrej.romanovsky@dignityhealth.org

Submitted: 01/12/2015; Accepted: 01/27/2015

http://dx.doi.org/10.1080/15384101.2015.1014150

This is an Open Access article distributed under the terms of the Creative Commons Attribution-Non-Commercial License (http://creativecommons.org/licenses/ by-nc/3.0/), which permits unrestricted non-commercial use, distribution, and reproduction in any medium, provided the original work is properly cited. The moral rights of the named author(s) have been asserted. 
not clear whether ROS play roles in fever and hypothermia, the 2 thermoregulatory responses to LPS. Riedel et al. ${ }^{12,13}$ has proposed that oxidative stress is a crucial component in the pathogenesis of LPS fever, but the data used to substantiate their proposal are difficult to interpret. Riedel et al. ${ }^{12,13}$ observed an attenuation of LPS fever by high doses of dithiotreitol, methylene blue, aspirin, or $\alpha$-lipolic acid and attributed fever-attenuating effects of these compounds to their free radical-scavenging action. However, all these compounds are nonselective radical scavengers; they also inhibit cyclooxygenase (COX), ${ }^{14}$ guanylyl cyclase, ${ }^{15}$ and secretory phospholipase $\mathrm{A}_{2}{ }^{16}$-important enzymes participating in the inflammatory response to LPS. Hence, the observed attenuation of LPS fever might have been due to inhibition of COX and other enzymes and not due to free radical scavenging.

Further complicating the issue, most exogenous ROS scavengers are water soluble and, therefore, can neutralize efficiently only substances dissolved in water (but not in lipids), whereas a large pool of free radicals accumulates in cellular membranes as lipoperoxides. ${ }^{17}$ These lipoperoxides are important mediators of oxidative stress, but they cannot be inactivated by conventional administration of water-soluble ROS scavengers. To dissect the roles of membrane ROS requires using lipophilic antioxidants.

Bilirubin is the end product of heme catabolism in mammals. This lipophilic molecule is generally considered a toxic waste that needs to be excreted. ${ }^{18,19}$ However, many studies have shown that bilirubin is a powerful antioxidant and efficient scavenger of lipoperoxide radicals in membranes. ${ }^{20,21}$ Hence, bilirubin can be used as a tool to inactivate water-insoluble, lipid-associated ROS. An established animal model to study the antioxidant activity of bilirubin in vivo is the Gunn rat model, ${ }^{22-24}$ named after $\mathrm{C}$. $\mathrm{H}$. Gunn, who characterized an autosomal recessive deficiency in uridine diphosphate glucuronyltransferase activity. ${ }^{25}$ This deficiency prevents the conjugation of bilirubin, which explains hyperbilirubinemia and neonatal jaundice in Gunn rats with the homozygous mutation $(\mathrm{J} / \mathrm{J})$, while heterozygous $(\mathrm{J} /+)$ rats are nonjaundiced. In $\mathrm{J} / \mathrm{J}$ rats, the increased antioxidant activity due to hyperbilirubinemia has been proposed to contribute to the protection against the harmful effects of hyperoxia ${ }^{22}$ and of angiotensin II. ${ }^{23}$

Continuing our studies of the thermoregulatory responses to LPS in mutant rats (Zucker, ${ }^{26,27}$ Otsuka Long-Evans Tokushima fatty, ${ }^{26,28}$ Koletsky, ${ }^{29}$ and Nagase analbuminemic ${ }^{30}$ ), the present work was focused on LPS-induced fever and hypothermia in J/J and J/+ Gunn rats. We also measured blood levels of biochemical markers of renal disfunction and hepatocyte damage in these rats following LPS administration.

\section{Results}

\section{LPS-induced fever and hypothermia in Gunn rats}

All experiments were conducted at an ambient temperature $\left(T_{a}\right)$ of $30^{\circ} \mathrm{C}$, which is within the thermoneutral zone for rats in our experimental setup. ${ }^{31}$ Two intravenous doses of LPS were used: the low $(10 \mu \mathrm{g} / \mathrm{kg})$ and the high $(1,000 \mu \mathrm{g} / \mathrm{kg})$. At $30^{\circ} \mathrm{C}$, rats respond to the low dose of LPS with a polyphasic fever, and to the high dose with hypothermia followed by fever. ${ }^{3}$ In the
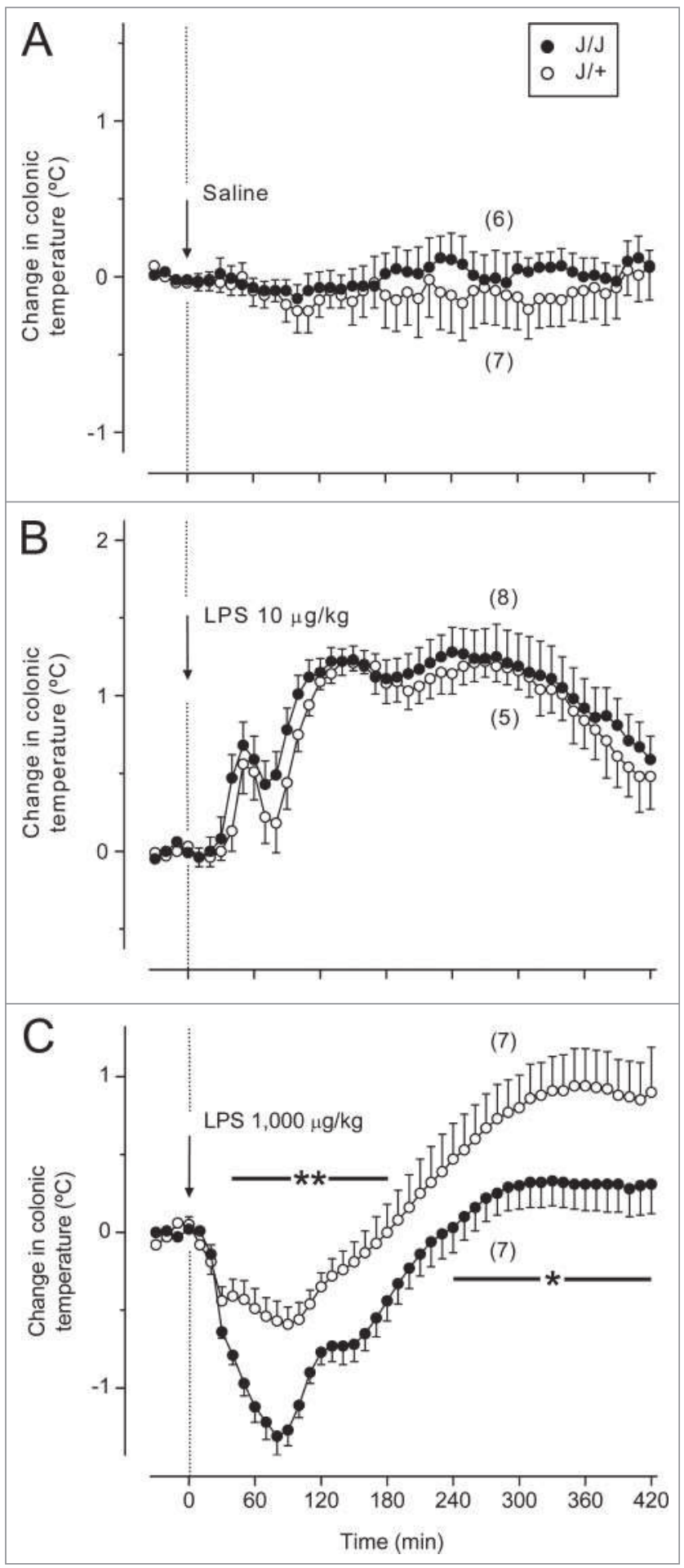

Figure 1. Deep (colonic) $T_{b}$ responses of $\mathrm{J} / \mathrm{J}$ and $\mathrm{J} /+$ rats to the low $(10 \mu \mathrm{g} / \mathrm{kg}$, iv) and high $(1,000 \mu \mathrm{g} / \mathrm{kg}$, iv) doses of LPS. (A) Administration of the vehicle (saline) does not affect $T_{b}$ in rats. (B) The low dose of LPS causes polyphasic fevers in $\mathrm{J} / \mathrm{J}$ and $\mathrm{J} / \mathrm{C}$ rats, without any significant differences between the genotypes. (C) In J/+ rats, the high dose of LPS induces early hypothermia followed by late fever. In J/J rats, the hypothermic response is exaggerated, and the subsequent febrile response is attenuated, as compared to the $\mathrm{J} /+$ controls. Time periods corresponding to significant intergenotype differences in the response to LPS are marked as ${ }^{*}(P<0.05)$ or ${ }^{* *}(P<0.01)$. 


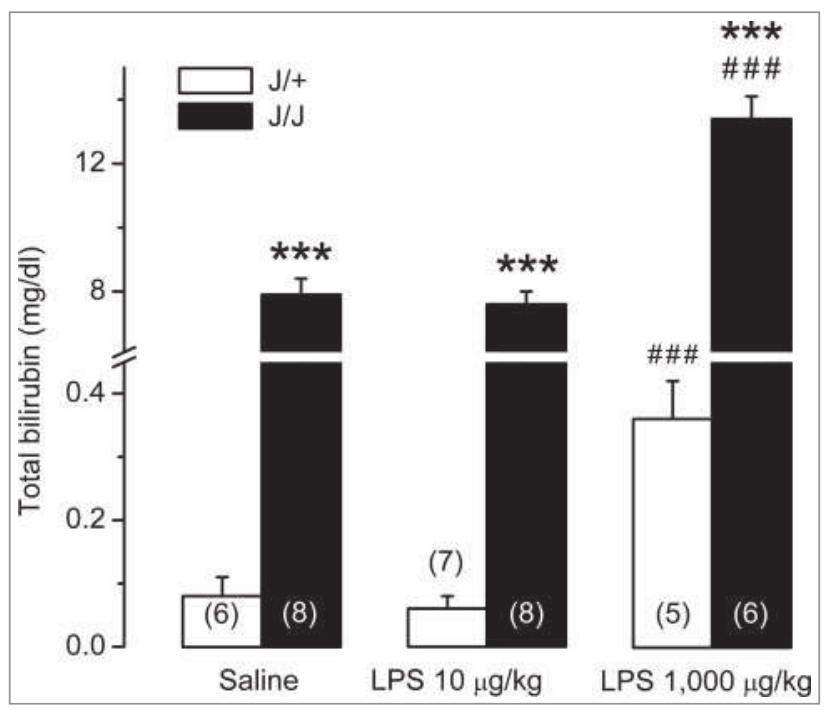

Figure 2. Blood bilirubin levels in $\mathrm{J} / \mathrm{J}$ and $\mathrm{J} /+$ rats. The total bilirubin level in blood plasma is dramatically higher in saline-treated $\mathrm{J} / \mathrm{J}$ rats than in saline-treated $\mathrm{J} /+$ controls. The low dose of LPS $(10 \mu \mathrm{g} / \mathrm{kg}$, iv) does not change the bilirubin level in either genotype. In response to the high dose of LPS $(1,000 \mu \mathrm{g} / \mathrm{kg}$, iv), the total bilirubin level increases in both genotypes, but remains substantially higher in $\mathrm{J} / \mathrm{J}$ rats than in $\mathrm{J} /+$ controls. ${ }^{* *}, P<0.001$, intergenotype difference in the response to LPS. $\# \#, P<0.001$, LPS vs. saline difference within the same genotype.

present study, the intravenous injection of saline did not affect the colonic temperature (an index of deep body temperature, $T_{b}$ ) in either $\mathrm{J} / \mathrm{J}$ or $\mathrm{J} /+$ Gunn rats (Fig. 1A). To the low dose of LPS, both $\mathrm{J} / \mathrm{J}$ and $\mathrm{J} /+$ rats responded with a triphasic fever, with peaks at $\sim 50,130$, and 280 min post-injection (Fig. 1B). There were no significant differences in the $T_{b}$ dynamics between the genotypes. To the high dose of LPS, J/+ rats responded with early hypothermia (nadir of $-0.5^{\circ} \mathrm{C}$ at $\sim 90$ min; $P=0.034$ vs. saline) followed by fever (peak of $0.9^{\circ} \mathrm{C}$ at $\sim 360 \mathrm{~min} ; P<0.001$ vs. saline) (Fig. 1C). The $T_{b}$ response of $\mathrm{J} / \mathrm{J}$ rats to the high dose of LPS was different from that of $\mathrm{J} /+$ rats: the early hypothermic response (nadir of $-1.2^{\circ} \mathrm{C}$ at $\sim 90 \mathrm{~min}, P<0.001$ vs. saline) was markedly exaggerated $(P \leq 0.004$ for $40-180 \mathrm{~min} v \mathrm{vs} . \mathrm{J} /+$ rats $)$, whereas the subsequent fever response (peak of $0.3^{\circ} \mathrm{C}, P=$ 0.039 vs. saline) was significantly attenuated $(P<0.05$ for 240 $420 \mathrm{~min}$ vs. $\mathrm{J} /+$ rats).

\section{Plasma bilirubin in Gunn rats}

Since LPS administration could induce liver failure, thereby causing (or exaggerating) hyperbilirubinemia, we examined blood bilirubin levels in $\mathrm{J} / \mathrm{J}$ and $\mathrm{J} /+$ rats under basal conditions and in LPS-induced systemic inflammation. As expected, the total plasma bilirubin level in saline-treated $\mathrm{J} / \mathrm{J}$ rats was higher (by 2 orders of magnitude) than that in $\mathrm{J} /+$ rats $(P<0.001$, Fig. 2). The low dose of LPS did not affect the total bilirubin level in either genotype. However, in response to the injection of the high dose of LPS, the total bilirubin level surged in both $\mathrm{J} /+$ and $\mathrm{J} / \mathrm{J}$ rats $(P<0.001$ vs. saline, for both genotypes). In $\mathrm{J} / \mathrm{J}$ rats treated with the high dose of LPS, the total plasma bilirubin level remained higher than in $\mathrm{J} /+$ controls $(P<0.001)$.
Renal disfunction and hepatic damage in Gunn rats after LPS administration

Plasma blood urea nitrogen (BUN) and creatinine levels were measured as markers of renal function. ${ }^{32}$ Neither of these markers differed significantly between saline-treated $\mathrm{J} / \mathrm{J}$ and $\mathrm{J} /+$ rats (Fig. 3). While administration of the low dose of LPS did not raise the renal disfunction markers, the high dose increased both BUN (Fig. 3A) and creatinine (Fig. 3B) in $\mathrm{J} / \mathrm{J}(P \leq 0.001$ for both BUN and creatinine) and $\mathrm{J} /+$ rats $(P=0.010$ for BUN; $P<0.001$ for creatinine) without any significant differences between the genotypes.

Plasma alanine aminotransferase (ALT), aspartate aminotransferase (AST), and gamma-glutamyl transferase (GGT)

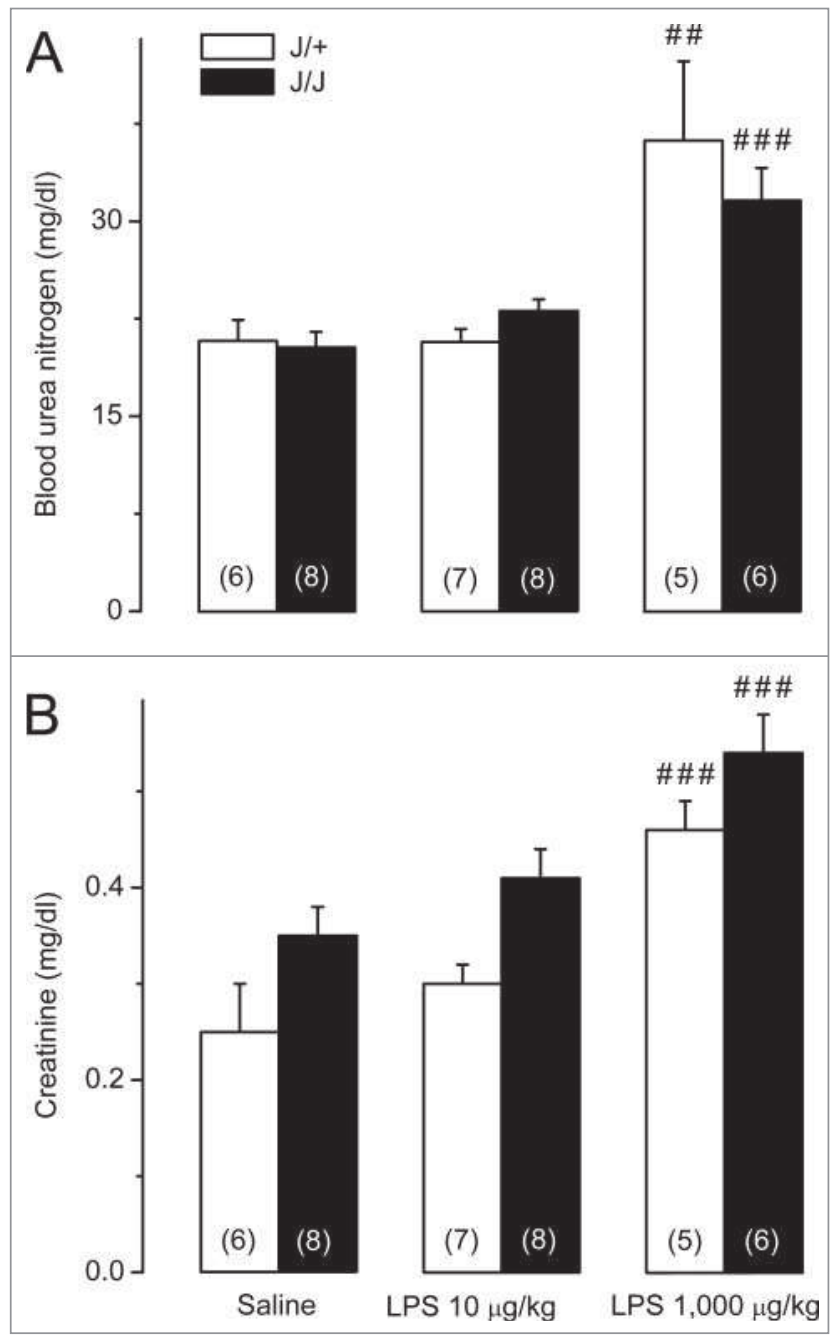

Figure 3. Biochemical markers of renal disfunction in $\mathrm{J} / \mathrm{J}$ and $\mathrm{J} /+$ rats. (A) Plasma BUN levels do not differ between saline-treated $\mathrm{J} / \mathrm{J}$ and $\mathrm{J} /+$ rats and remain unchanged after administration of the low dose of LPS (10 $\mu \mathrm{g} / \mathrm{kg}$, iv). The high dose of LPS $(1,000 \mu \mathrm{g} / \mathrm{kg}$, iv) raises BUN in both genotypes, without any intergenotype difference. (B) Plasma creatinine levels do not differ between saline-treated $J / J$ and $J /+$ rats and remain unchanged after administration of the low dose of LPS. The high dose of LPS raises plasma creatinine in both genotypes, without any intergenotype difference. Within each genotype, significant differences in the response to LPS (as compared to saline) are marked as ${ }^{\# \#}(P<0.01)$ or $^{\# \# \#(}(P<0.001)$. 
were used to assess hepatocyte damage. ${ }^{33}$ Activities of all 3 enzymes were within the normal range in $\mathrm{J} / \mathrm{J}$ and $\mathrm{J} /+$ rats after the injection of saline or the low dose of LPS (Fig. 4). When LPS was administered at the high dose, rats exhibited marked surges in ALT $(P=0.019$ for $\mathrm{J} /+; P=0.015$ for $\mathrm{J} / \mathrm{J}$; Fig. $4 \mathrm{~A})$, AST $(P=0.021$ for $\mathrm{J} /+; P=0.041$ for $\mathrm{J} / \mathrm{J}$; Fig. 4B), and GGT $(P<0.001$ for both genotypes; Fig. $4 \mathrm{C})$. In $\mathrm{J} / \mathrm{J}$ rats, the surge was blunted for GGT $(P=$ $0.042)$ and tended to be reduced for ALT $(P=0.208)$ and AST $(P=0.179)$, as compared to $\mathrm{J} /+$ rats.
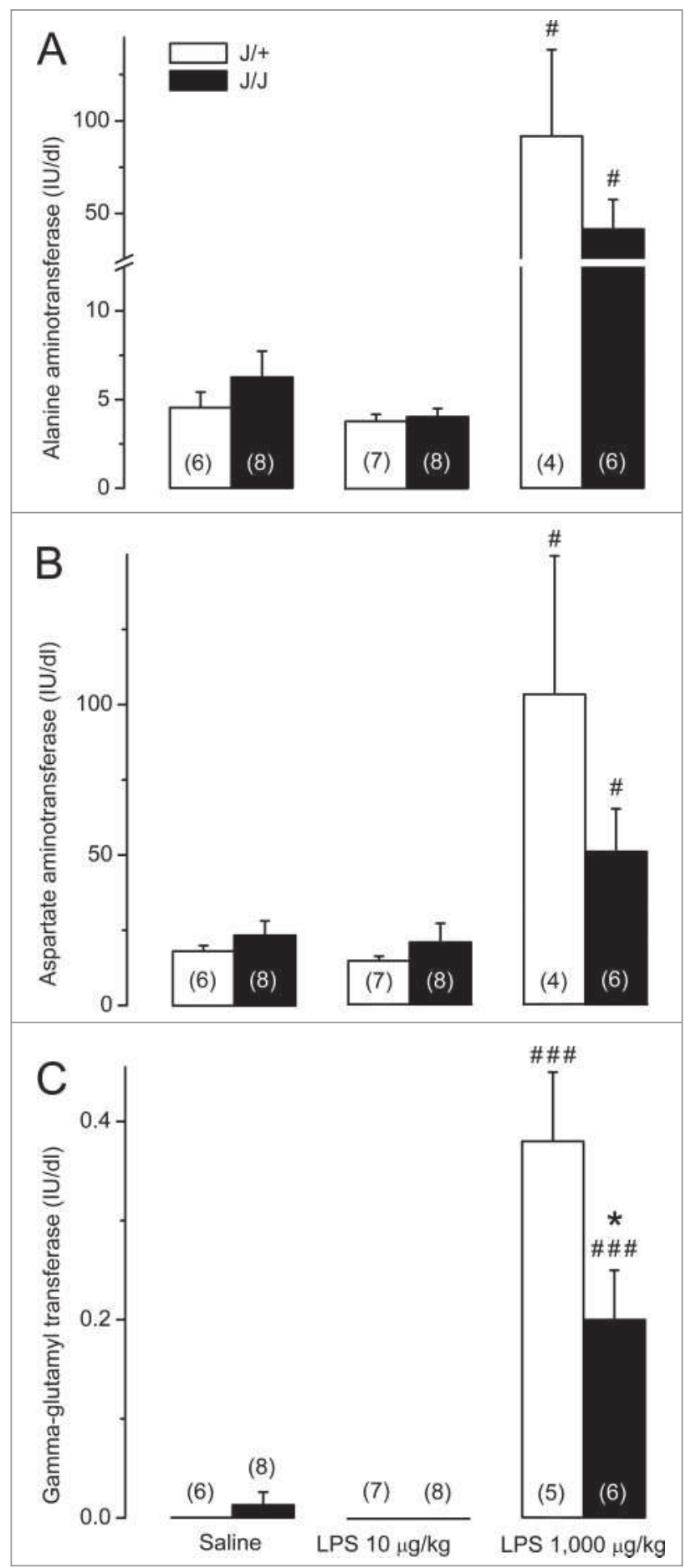

\section{Discussion}

LPS hypothermia is exaggerated in hyperbilirubinemic

\section{Gunn rats}

The novel - and unexpected-finding of the present study is that hyperbilirubinemia in Gunn rats was associated with an exaggerated hypothermic response to LPS (Fig. 1C). This exaggeration is unlikely to be due to an attenuation of the ROS-mediated inflammatory signaling by bilirubin. Indeed, attenuating inflammatory signaling should decrease (not increase) the hypothermic response. Furthermore, while bilirubin, a potent ROS scavenger, has been shown repeatedly to attenuate inflammatory signaling, ${ }^{34-36}$ we did not find any literature data suggesting that it can amplify inflammatory signaling. An alternative, more plausible, mechanism of the exaggeration of LPS hypothermia might be an action of bilirubin on thermoeffectors. Gunn rats respond to administration of exogenous bilirubin (that further increases their hyperbilirubinemia) with a pronounced $\left(\sim 3^{\circ} \mathrm{C}\right)$ drop in $T_{b} .{ }^{37}$ This drop is likely to reflect decreased thermogenesis, because bilirubin has been demonstrated to depress mitochondrial respiration in liver, heart, and brain tissues in vitro. ${ }^{38-40}$ And although brown adipose tissue (the main source of nonshivering, temperature-driven thermogenesis in rodents ${ }^{41}$ ) has not been studied, bilirubin is likely to have the same effect in brown fat as in all other tissues studied. A profound decrease in the threshold $T_{b}$ for activation of thermogenesis is the principle autonomic thermoeffector mechanism of LPSinduced hypothermia. ${ }^{42}$ Although skin vasodilation is not the main mechanism of LPS-induced hypothermia, ${ }^{42}$ increased skin vasodilation in $\mathrm{J} / \mathrm{J}$ rats could have contributed to their enhanced hypothermic response to LPS as well. Hyperbilirubinemia has been shown to attenuate the pressor effects of angiotensin II in Gunn rats $^{23}$ and to lower angiotension IIdependent hypertension in mice, ${ }^{43,44}$ the latter effect being attributed to the blockade of superoxide production and possibly to enhanced vasorelaxation. ${ }^{43}$ In a study conducted in humans, ${ }^{45}$ hyperbilirubinemia exaggerated endotheliumdependent vasodilation of the brachial artery, which supplies subcutaneous and cutaneous tissues of the arm.

Figure 4. Biochemical markers of hepatocyte damage in $\mathrm{J} / \mathrm{J}$ and $\mathrm{J} /+$ rats. (A) Plasma ALT levels do not differ between saline-treated $\mathrm{J} / \mathrm{J}$ and $\mathrm{J} /+$ rats and remain unchanged after the administration of the low dose of LPS (10 $\mu \mathrm{g} / \mathrm{kg}$, iv). In response to the high dose of LPS $(1,000 \mu \mathrm{g} / \mathrm{kg}$, iv), plasma ALT surges in both genotypes, but this surge tends to be blunted in $\mathrm{J} / \mathrm{J}$ rats (as compared to the $\mathrm{J} /+$ controls). (B) Plasma AST levels do not differ between $\mathrm{J} / \mathrm{J}$ and $\mathrm{J} / \mathrm{+}$ rats after administration of saline or LPS at the low dose. In response to the high dose of LPS, AST surges in both genotypes, but the surge tends to be blunted in $\mathrm{J} / \mathrm{J}$ rats). (C) Plasma GGT levels are near the detection threshold in $\mathrm{J} / \mathrm{J}$ and $\mathrm{J} /+$ rats after administration of saline or the low dose of LPS. Plasma GGT rises in both genotypes in response to the high dose of LPS, but the rise is significantly reduced in $\mathrm{J} / \mathrm{J}$ rats. A significant $(P<0.05)$ intergenotype difference in the response to LPS is marked with * Within each genotype, significant differences in the response to LPS (as compared to saline) are marked as

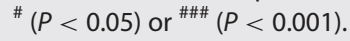




\section{Hyperbilirubinemia differently affects fevers caused by low vs. high doses of LPS}

The same action (or actions) on thermoeffectors could be responsible for the attenuation of the late fever response to the high dose of LPS in J/J rats, which was also observed in the present study (Fig. 1C). Since the thermoregulatory response to the high dose is a combination of early hypothermia and late fever, the exaggeration of the hypothermic response (and the resultant prolongation of this response) may be difficult to distinguish from an independent attenuation of the fever response.

Interestingly, the fever response to the low dose of LPS was not affected by hyperbilirubinemia in Gunn rats in our study (Fig. 1B), which is another novel observation. It suggests that ROS signaling is not involved in the febrile response, at least not to weak stimuli. It also extends our earlier conclusion that NO, a known ROS, is not involved in the pyrogenic signaling of LPS. ${ }^{46}$ However, it cannot be ruled out that hyperbilirubinemia blocks pro-pyretic ROSmediated signaling triggered by high doses of LPS, i.e., doses that cause a prominent ROS response. For example, high doses of LPS (those that lead to shock and hypothermia) induce overexpression of the inducible $\mathrm{NO}$ synthase isoform, ${ }^{47,48}$ thus leading to the massive production of $\mathrm{NO}$ in rats. ${ }^{48,49}$ In contrast, lower (pyrogenic) doses of LPS suppress NO synthesis, possibly by inhibiting constitutive NO synthase isoforms. ${ }^{50,51}$

This proposed antipyretic, ROS-mediated action of bilirubin upon fevers caused by high doses of LPS would agree with several studies. ${ }^{12,13,52,53}$ In these studies, the febrile response to LPS was attenuated by antioxidants, whether water-soluble (such as methylene blue, dithiothreitol, aspirin, lipoic acid, natural antioxidants purified from spinach, or apocynin) ${ }^{12,13,52}$ or lipid-soluble (such as resveratrol and lipoic acid). ${ }^{12,53}$ Of those 4 studies, one ${ }^{53}$ involved administering an extremely high dose of LPS $(4 \mathrm{mg} / \mathrm{kg})$ to rats, whereas 3 other studies ${ }^{12,13,52}$ used $1-100 \mu \mathrm{g} / \mathrm{kg}$ doses in rabbits, the species that is $\sim 100$ times more sensitive to LPS than rats. Interestingly, the high sensitivity of rabbits to LPS has been explained by the agile production of ROS in this species without a concomitant increase in the protective antioxidative enzymes. ${ }^{54}$ On the other hand, the relative resistance of rats to LPS has been ascribed to an increased activity of the endogenous antioxidant systems. ${ }^{54,55}$ It should also be noted that several ROS scavengers used in the studies discussed above ${ }^{12,13,52,53}$ inhibit the synthesis of $\mathrm{PGE}_{2}$, the principle mediator of fever, ${ }^{56,57}$ by acting on $\mathrm{COX}^{14,58-60}$ and secretory phospholipase $A_{2}{ }^{16}$ However, it is unlikely that all the reports of the fever-attenuating effects of ROS scavengers reflect an action on PG synthesis, because the antipyretic effect has been reported for multiple, structurally unrelated compounds. Furthermore, bilirubin (the focus of the present study) and several other ROS scavengers have not been shown to inhibit $\mathrm{PGE}_{2}$ synthesis, definitely not by an action independent from ROS neutralization. There are several studies showing that antioxidants (e.g., bilirubin, methylene blue, and apocynin) did not affect $\mathrm{PGE}_{2}$ synthesis or COX-2 expression in various models. ${ }^{61-64}$
Hyperbilirubinemia attenuates LPS-induced hepatic damage in Gunn rats

Compared to $\mathrm{J} /+$ rats, $\mathrm{J} / \mathrm{J}$ rats responded to the high dose of LPS with a blunted surge in GGT (Fig. 4C). The LPS-induced surges in 2 other transferases studied (ALT and AST) tended to be reduced, but the magnitude of the reduction did not reach the level of statistical significance (Fig. 4A, B). The revealed high sensitivity of GGT to hyperbilirubinemia was expected. Indeed, GGT is also a marker of oxidative stress, ${ }^{65}$ and the blood concentration of this enzyme is inversely related to the blood antioxidant activity. ${ }^{66,67}$ The observed effect of hyperbilirubinemia on the LPS-induced GGT response and the tendencies revealed (with regard to ALT and AST responses) agree with the previously reported protective action of bilirubin in endotoxin shock ${ }^{68}$ and other pathological conditions. ${ }^{69,70}$

The cytoprotective action of bilirubin is likely to be due to the suppression of oxidative stress, either directly (by scavenging ROS) or indirectly (by inhibiting the expression of free radicalgenerating enzymes, such as NO synthase and nicotinamide adenine dinucleotide phosphate oxidase) ${ }^{68}$ By either mechanism, bilirubin potently suppresses the accumulation of lipophilic ROS in cell membranes, ${ }^{20,21}$ and the decreased accumulation of ROS prevents cell apoptosis and tissue injury associated with oxidative stress. ${ }^{70}$ Yet another mechanism of tissue protection by bilirubin in LPS-induced systemic inflammation could be the exaggeration of hypothermia. Hypothermia is an adaptive, active thermoregulatory response to high doses of LPS and other strong inflammatory stimuli. ${ }^{71}$ It involves cold-seeking behavior ${ }^{42,72,73}$ and is thought to be aimed at decreasing the metabolic requirements of tissues. $^{3,71}$ In our studies, ${ }^{74,75}$ when rats were either allowed to select their preferred low $T_{a}$ or maintained at a lower $T_{a}$ chosen by the investigators during endotoxin shock or Escherichia coli infection, their survival rates increased. At least in some cases, the increased survival was coupled with a suppressed surge in ALT. ${ }^{75}$

Whereas markers of hepatocyte damage were affected by hyperbilirubinemia in the present study, markers of renal disfunction (BUN and creatinine) were not (Fig. 3), thus suggesting the lack of tissue protection in the kidneys. This is despite the fact that bilirubin has been shown to play a protective role in the kidneys, e.g., in diabetic nephropathy. ${ }^{76}$ The observed in the present study tissue specificity for the protective action of bilirubin may relate to the fact that the liver is the principal site for bilirubin metabolism. Due to this, bilirubin concentration in hepatocytes should be higher than anywhere else in the body, including renal epitheliocytes. Although bilirubin conjugation is defective in $\mathrm{J} / \mathrm{J}$ rats, ${ }^{25}$ and these animals excrete only traces of bilirubin in the bile, ${ }^{77}$ they still have more than a 2 -fold-higher concentration of bilirubin in the liver than in the kidneys. ${ }^{78}$

\section{Summary and conclusions}

We showed that hyperbilirubinemia in J/J Gunn rats was associated with a marked exaggeration of the early hypothermic response to the high dose of LPS $(1,000 \mu \mathrm{g} / \mathrm{kg})$, presumably through a direct inhibition of nonshivering thermogenesis by bilirubin and possibly also through a direct vasodilatatory action of bilirubin in the skin. This novel, hypothermia-exaggerating effect 
might be responsible, at least in part, for the observed tendency of $J / J$ rats to respond to the high dose of LPS with attenuated hepatic damage.

Hyperbilirubinemia in Gunn rats was also associated with a deep attenuation of the late febrile response to the high $(1,000 \mu \mathrm{g} / \mathrm{kg})$ dose of LPS, but did not attenuate the fever response to the low $(10 \mu \mathrm{g} / \mathrm{kg})$ dose. The attenuation of the fever response to $1,000 \mu \mathrm{g} / \mathrm{kg}$ could be due to either direct actions of bilirubin on thermoeffectors (inhibition of nonshivering thermogenesis and induction of skin vasodilation) or the ROS-scavenging action of bilirubin attenuating pyrogenic signaling. However, the experiments with $10 \mu \mathrm{g} / \mathrm{kg}$ strongly suggest that ROS signaling is not involved in the fever response to low doses of LPS.

\section{Materials and Methods}

\section{Animals}

Adult male J/J and J/+ Gunn rats were obtained from Harlan. They were housed in cages kept in a rack equipped with a Smart Bio-Pack ventilation system and Thermo-Pak temperature control system (Allentown Caging Equipment); the temperature of incoming air was maintained at $28^{\circ} \mathrm{C}$. Standard rodent chow and tap water were available ad libitum. The room was on a $12 \mathrm{~h}$ light/dark cycle (lights on at 7:00 A.M.). The rats were housed in groups until they were subjected to surgery, after which they were caged singly. Animals were extensively handled and habituated to staying in wire-mesh conical confiners, which were later used in the experiments. At the time of the experiments the rats weighed 200-245 g, there was no significant difference between the body mass of rats of different genotypes. All procedures were conducted under protocols approved by the Legacy Health System and St. Joseph's Hospital and Medical Center Animal Care and Use Committees.

\section{Intravenous catheterization}

Four days before an experiment, each rat was implanted with a jugular catheter. The procedure was performed under ketaminexylazine-acepromazine $(55.6,5.5$, and $1.1 \mathrm{mg} / \mathrm{kg}$ ip, respectively) anesthesia and antibiotic (enrofloxacin, $1.1 \mathrm{mg} / \mathrm{kg} \mathrm{sc}$ ) protection. During the surgery, a rat was heated with a Deltaphase isothermal pad (Braintree Scientific). A small longitudinal incision was made on the ventral surface of the neck, left to the trachea. The left jugular vein was exposed, freed from its surrounding connective tissue, and ligated. A silicone catheter (ID $0.5 \mathrm{~mm}$, OD $0.9 \mathrm{~mm})$ filled with heparinized saline $(50 \mathrm{U} / \mathrm{ml})$ was passed into the superior vena cava through the jugular vein and secured in place with ligatures. The free end of the catheter was knotted, tunneled under the skin to the nape, and exteriorized. The wound on the ventral surface of the neck was sutured. To prevent postsurgical hypothermia, the animals were allowed to recover from anesthesia in an environmental chamber (model 3940; Forma Scientific) set to $28^{\circ} \mathrm{C}$. The intravenous catheters were flushed with heparinized saline every other day.

\section{Experimental setup}

On the day of the experiment, each rat was placed in a confiner and equipped with a copper-constantan thermocouple (Omega Engineering) to measure colonic temperature (a measure of deep $T_{b}$ ). The thermocouple was inserted in the colon $10 \mathrm{~cm}$ deep beyond the anal sphincter and fixed to the tail with a loop of adhesive tape. To record $T_{b}$ data, the thermocouple was plugged into a data logger (Cole-Parmer) connected to a computer. The rat in its confiner was then placed in an environmental chamber (Forma Scientific) set to a $T_{a}$ of $30^{\circ} \mathrm{C}$, which is a neutral $T_{a}$ for rats in this setup. ${ }^{31}$ The implanted venous catheter was extended with a length of PE50 tubing filled with saline. The extension was passed through a wall port and connected to a syringe filled with LPS or saline.

\section{LPS administration}

LPS from E. coli 0111:B4 was purchased from Sigma-Aldrich. A stock suspension of LPS $(5 \mathrm{mg} / \mathrm{ml})$ in pyrogen-free saline was stored at $-20^{\circ} \mathrm{C}$. On the day of the experiment, the stock was diluted to a final concentration of either 10 or $1,000 \mu \mathrm{g} / \mathrm{ml}$. The diluted LPS suspension or saline was injected in a bolus $(1 \mathrm{ml} /$ $\mathrm{kg}$ ) through the extension of the venous catheter. Deep $T_{b}$ was monitored for $7 \mathrm{~h}$ after the injection.

\section{Biochemical assays}

To determine blood levels of total bilirubin and markers of renal disfunction and hepatocyte damage, rats were anesthetized with ketamine-xylazine-acepromazine $(5.6,0.6$, and $0.1 \mathrm{mg} / \mathrm{kg}$ ip, respectively) $24 \mathrm{~h}$ after LPS injection, and their arterial blood $(5 \mathrm{ml})$ was collected by cardiac (left ventricle) puncture. The blood was immediately transferred to EDTA-containing Vacutainer tubes (Beckton Dickinson). The plasma was separated by centrifugation, aliquoted, and stored at $-80^{\circ} \mathrm{C}$ until biological assays were performed. All samples were sent to the Legacy Central Laboratory, Portland, OR. ALT and AST activities in serum were determined according to the method of Reitman and Frankel, ${ }^{79}$ whereas GGT levels were assessed by the Szasz method. ${ }^{80}$ Total plasma bilirubin level, BUN, and creatinine concentration was determined according to the method described by Jendrassik et al., ${ }^{81}$ Patton and Crouch, ${ }^{82}$ and Van Pilsum, ${ }^{83}$ respectively.

\section{Statistical analysis}

The $T_{b}$ responses were compared by 2-way ANOVA followed by the Fisher least significant difference test by using Sigmaplot 11.0 (Systat Software). Blood levels of bilirubin and biochemical markers of renal and hepatic disfunction were compared with Student's $t$ test. The effects were considered significant when $P<$ 0.05 . The data are reported as means \pm SE.

\section{Disclosure of Potential Conflicts of Interest}

No potential conflicts of interest were disclosed. 


\section{Funding}

This research has been supported in part by the National Institutes of Health (grant R01NS41233 to AAR) and the
Hungarian Scientific Research Fund (grant PD 105532 to AG). AG acknowledges the Janos Bolyai Scholarship of the Hungarian Academy of Sciences.

\section{References}

1. Wilhelms DB, Kirilov M, Mirrasekhian E, Eskilsson A, Kugelberg UO, Klar C, Ridder DA, Herschman HR, Schwaninger M, Blomqvist A, et al. Deletion of prostaglandin E2 synthesizing enzymes in brain endothelial cells attenuates inflammatory fever. J Neurosci 2014; 34:11684-90; PMID:25164664; http://dx.doi.org/ 10.1523/JNEUROSCI.1838-14.2014

2. Roth J, Blatteis CM. Mechanisms of Fever production and lysis: lessons from experimental LPS Fever. Compr Physiol 2014; 4:1563-604; PMID:25428854; http:// dx.doi.org/10.1002/cphy.c130033

3. Romanovsky AA, Almeida MC, Aronoff DM, Ivanov AI, Konsman JP, Steiner AA, Turek VF. Fever and hypothermia in systemic inflammation: recent discoveries and revisions. Front Biosci 2005; 10:2193-216; PMID:15970487; http://dx.doi.org/10.2741/1690

4. Saper CB, Romanovsky AA, Scammell TE. Neural circuitry engaged by prostaglandins during the sickness syndrome. Nat Neurosci 2012; 15:1088-95; PMID:22837039; http://dx.doi.org/10.1038/nn.3159

5. Steiner AA, Ivanov AI, Serrats J, Hosokawa H, Phayre AN, Robbins JR, Roberts JL, Kobayashi S, Matsumura $\mathrm{K}$, Sawchenko PE, et al. Cellular and molecular bases of the initiation of fever. PLoS Biol 2006; 4:e284; PMID:16933973; http://dx.doi.org/10.1371/journal. pbio.0040284

6. Steiner AA, Branco LG. Fever and anapyrexia in systemic inflammation: intracellular signaling by cyclic nucleotides. Front Biosci 2003; 8:s1398-408; PMID:12957844; http://dx.doi.org/10.2741/1188

7. Forman HJ, Torres M. Reactive oxygen species and cell signaling: respiratory burst in macrophage signaling. Am J Respir Crit Care Med 2002; 166:S4-8; PMID:12471082; $\quad$ http://dx.doi.org/10.1164/ rccm. 2206007

8. Hampton MB, Kettle AJ, Winterbourn CC. Inside the neutrophil phagosome: oxidants, myeloperoxidase, and bacterial killing. Blood 1998; 92:3007-17; PMID:9787133

9. Babior BM, Kipnes RS, Curnutte JT. Biological defense mechanisms. The production by leukocytes of superoxide, a potential bactericidal agent. J Clin Invest 1973; 52:741-4; PMID:4346473; http://dx.doi.org/ $10.1172 / \mathrm{JCI} 107236$

10. Lander HM. An essential role for free radicals and derived species in signal transduction. FASEB J 1997; 11:118-24; PMID:9039953

11. Thannickal VJ, Fanburg BL. Reactive oxygen species in cell signaling. Am J Physiol Lung Cell Mol Physiol 2000; 279:L1005-28; PMID:11076791

12. Riedel W, Lang U, Oetjen U, Schlapp U, Shibata M. Inhibition of oxygen radical formation by methylene blue, aspirin, or alpha-lipoic acid, prevents bacteriallipopolysaccharide-induced fever. Mol Cell Biochem 2003; 247:83-94; PMID:12841635; http://dx.doi.org/ 10.1023/A:1024142400835

13. Riedel W, Maulik G. Fever: an integrated response of the central nervous system to oxidative stress. Mol Cell Biochem 1999; 196:125-32; PMID:10448911; http:// dx.doi.org/10.1023/A:1006936111474

14. Milton AS. Modern views on the pathogenesis of fever and the mode of action of antipyretic drugs. J Pharm Pharmacol 1976; 28:393-9; PMID:6743; http://dx. doi.org/10.1111/j.2042-7158.1976.tb04186.x

15. Gruetter CA, Kadowitz PJ, Ignarro LJ. Methylene blue inhibits coronary arterial relaxation and guanylate cyclase activation by nitroglycerin, sodium nitrite, and amyl nitrite. Can J Physiol Pharmacol 1981; 59:150-6; PMID:6112057; http://dx.doi.org/10.1139/y81-025

16. Jameel NM, Shekhar MA, Vishwanath BS. Alphalipoic acid: an inhibitor of secretory phospholipase A2 with anti-inflammatory activity. Life Sci 2006; 80:14653; PMID:17011589; http://dx.doi.org/10.1016/j. Ifs.2006.08.032

17. Niki E. Antioxidants in relation to lipid peroxidation. Chem Phys Lipids 1987; 44:227-53; PMID:3311418; http://dx.doi.org/10.1016/0009-3084(87)90052-1

18. McDonagh AF. Controversies in bilirubin biochemistry and their clinical relevance. Semin Fetal Neonatal Med 2010; 15:141-7; PMID:19932645; http://dx.doi. org/10.1016/j.siny.2009.10.005

19. Brites D. Bilirubin injury to neurons and glial cells: new players, novel targets, and newer insights. Semin Perinatol 2011; 35:114-20; PMID:21641483; http:// dx.doi.org/10.1053/j.semperi.2011.02.004

20. Sedlak TW, Saleh M, Higginson DS, Paul BD, Juluri KR, Snyder SH. Bilirubin and glutathione have complementary antioxidant and cytoprotective roles. Proc Natl Acad Sci U S A 2009; 106:5171-6; PMID:19286972; http://dx.doi.org/10.1073/ pnas.0813132106

21. Stocker R, Yamamoto Y, McDonagh AF, Glazer AN, Ames BN. Bilirubin is an antioxidant of possible physiological importance. Science 1987; 235:1043-6; PMID:3029864; http://dx.doi.org/ 10.1126/science. 3029864

22. Dennery PA, McDonagh AF, Spitz DR, Rodgers PA, Stevenson DK. Hyperbilirubinemia results in reduced oxidative injury in neonatal Gunn rats exposed to hyperoxia. Free Radic Biol Med 1995; 19:395-404; PMID:7590389; http://dx.doi.org/10.1016/08915849(95)00032-S

23. Pflueger A, Croatt AJ, Peterson TE, Smith LA, d'Uscio LV, Katusic ZS, Nath KA. The hyperbilirubinemic Gunn rat is resistant to the pressor effects of angiotensin II. Am J Physiol Renal Physiol 2005; 288:F552-8; PMID:15536166; http://dx.doi.org/10.1152/ ajprenal.00278.2004

24. Wallner M, Antl N, Rittmannsberger B, Schreidl S, Najafi K, Mullner E, Molzer C, Ferk F, Knasmuller S, Marculescu R, et al. Anti-genotoxic potential of bilirubin in vivo: damage to DNA in hyperbilirubinemic human and animal models. Cancer Prev Res (Phila) 2013; 6:1056-63; PMID:23983086; http://dx.doi.org/ 10.1158/1940-6207.CAPR-13-0125

25. Gunn $\mathrm{CH}$. Hereditary acholuric jaundice: in a new mutant strain of rats. Journal of Heredity 1938 29:137-9.

26. Ivanov AI, Kulchitsky VA, Romanovsky AA. Does obesity affect febrile responsiveness? Int J Obes Relat Metab Disord 2001; 25:586-9; PMID:11319666; http://dx.doi.org/10.1038/sj.ijo.0801523

27. Ivanov AI, Romanovsky AA. Fever responses of Zucker rats with and without fatty mutation of the leptin receptor. Am J Physiol Regul Integr Comp Physiol 2002; 282:R311-6; PMID:11742853

28. Ivanov AI, Kulchitsky VA, Romanovsky AA. Role for the cholecystokinin-a receptor in fever: a study of a mutant rat strain and a pharmacological analysis. J Physiol 2003; 547:941-9; PMID:12562931; http://dx doi.org/10.1113/jphysiol.2002.033183

29. Steiner AA, Dogan MD, Ivanov AI, Patel S, Rudaya AY, Jennings DH, Orchinik M, Pace TW, O'Connor K A, Watkins LR, et al. A new function of the leptin receptor: mediation of the recovery from lipopolysaccharide-induced hypothermia. FASEB J 2004; 18:1949-51; PMID: 15388670

30. Ivanov AI, Steiner AA, Patel S, Rudaya AY, Romanovsky AA. Albumin is not an irreplaceable carrier for amphipathic mediators of thermoregulatory responses to LPS: compensatory role of alpha1-acid glycoprotein. Am J Physiol Regul Integr Comp Physiol 2005; 288: R872-8; PMID:15576666; http://dx.doi.org/10.1152/ ajpregu.00514.2004
31. Romanovsky AA, Ivanov AI, Shimansky YP. Selected contribution: ambient temperature for experiments in rats: a new method for determining the zone of thermal neutrality. J Appl Physiol (1985) 2002; 92:2667-79; PMID: 12015388

32. Ramesh G, Zhang B, Uematsu S, Akira S, Reeves WB. Endotoxin and cisplatin synergistically induce renal dysfunction and cytokine production in mice. Am J Physiol Renal Physiol 2007; 293:F325-32; PMID:17494092; http://dx.doi.org/10.1152/ ajprenal.00158.2007

33. Muftuoglu MA, Aktekin A, Ozdemir NC, Saglam A. Liver injury in sepsis and abdominal compartment syndrome in rats. Surg Today 2006; 36:519-24; PMID:16715421; http://dx.doi.org/10.1007/s00595006-3196-7

34. Kawamura K, Ishikawa K, Wada Y, Kimura S, Matsumoto H, Kohro T, Itabe H, Kodama T, Maruyama Y. Bilirubin from heme oxygenase-1 attenuates vascular endothelial activation and dysfunction. Arterioscler Thromb Vasc Biol 2005; 25:155-60; PMID:15499042

35. Kadl A, Pontiller J, Exner M, Leitinger N. Single bolus injection of bilirubin improves the clinical outcome in a mouse model of endotoxemia. Shock 2007; 28:5828; PMID: 17577133

36. Kasap B, Soylu A, Ertoy Baydar D, Kiray M, Tugyan K, Kavukcu S. Protective effects of bilirubin in an experimental rat model of pyelonephritis. Urology 2012; 80:1389 e17-22; PMID:22995569; http://dx. doi.org/10.1016/j.urology.2012.07.029

37. Krukow N, Brodersen R. Toxic effects in the Gunn rat of combined treatment with bilirubin and orotic acid. Acta Paediatr Scand 1972; 61:697-703; PMID:5080650; http://dx.doi.org/10.1111/j.16512227.1972.tb15969.x

38. Kamisaka K, Gatmaitan Z, Moore CL, Arias IM. Ligandin reverses bilirubin inhibition of liver mitochondrial respiration in vitro. Pediatr Res 1975; 9:9035; PMID:1196709; http://dx.doi.org/10.1203/ 00006450-197512000-00007

39. Mustafa MG, Cowger ML, King TE. Effects of bilirubin on mitochondrial reactions. J Biol Chem 1969; 244:6403-14; PMID:4982202

40. Noir BA, Boveris A, Garaza Pereira AM, Stoppani AO. Bilirubin: a multi-site inhibitor of mitochondrial respiration. FEBS Lett 1972; 27:270-4; PMID:4664225; http://dx.doi.org/10.1016/0014-5793(72)80638-0

41. Cannon B, Nedergaard J. Brown adipose tissue: function and physiological significance. Physiol Rev 2004; 84:277-359; PMID:14715917; http://dx.doi.org/ 10.1152/physrev.00015.2003

42. Romanovsky AA, Shido O, Sakurada S, Sugimoto N, Nagasaka T. Endotoxin shock: thermoregulatory mechanisms. Am J Physiol 1996; 270:R693-703; PMID:8967396

43. Stec DE, Storm MV, Pruett BE, Gousset MU. Antihypertensive actions of moderate hyperbilirubinemia: role of superoxide inhibition. Am J Hypertens 2013; 26:918-23; PMID:23482378; http://dx.doi.org/ 10.1093/ajh/hpt038

44. Vera T, Granger JP, Stec DE. Inhibition of bilirubin metabolism induces moderate hyperbilirubinemia and attenuates ANG II-dependent hypertension in mice. Am J Physiol Regul Integr Comp Physiol 2009; 297: R738-43; PMID:19571206; http://dx.doi.org/ 10.1152/ajpregu.90889.2008

45. Maruhashi T, Soga J, Fujimura N, Idei N, Mikami S, Iwamoto Y, Kajikawa M, Matsumoto T, Kihara Y, Chayama K, et al. Hyperbilirubinemia, augmentation of endothelial function, and decrease in oxidative stress in Gilbert syndrome. Circulation 2012; 126:598-603; PMID:22773454; http://dx.doi.org/10.1161/ CIRCULATIONAHA.112.105775 
46. Steiner AA, Rudaya AY, Ivanov AI, Romanovsky AA. Febrigenic signaling to the brain does not involve nitric oxide. $\mathrm{Br} \mathrm{J}$ Pharmacol 2004; 141:1204-13; PMID:15006900; $\quad$ http://dx.doi.org/10.1038/sj. bjp.0705713

47. Titheradge MA. Nitric oxide in septic shock. Biochim Biophys Acta 1999; 1411:437-55; PMID:10320674; http://dx.doi.org/10.1016/S0005-2728(99)00031-6

48. Bachetti T, Pasini E, Suzuki H, Ferrari R. Species-specific modulation of the nitric oxide pathway after acute experimentally induced endotoxemia. Crit Care Med 2003; 31:1509-14; PMID:12771626; http://dx.doi. org/10.1097/01.CCM.0000063269.35714.7E

49. Jourd'heuil D, Gray L, Grisham MB. S-nitrosothiol formation in blood of lipopolysaccharide-treated rats. Biochem Biophys Res Commun 2000; 273:22-6; PMID:10873557; $\quad$ http://dx.doi.org/10.1006/ bbrc. 2000.2892

50. Riedel W. Antipyretic role of nitric oxide during endotoxin-induced fever in rabbits. Int J Tissue React 1997; 19:171-8; PMID:9506319

51. Steiner AA, Antunes-Rodrigues J, McCann SM, Branco LG. Antipyretic role of the NO-cGMP pathway in the anteroventral preoptic region of the rat brain. Am J Physiol Regul Integr Comp Physiol 2002; 282 R584-93; PMID: 11792670

52. Lomnitski L, Carbonatto M, Ben-Shaul V, Peano S, Conz A, Corradin L, Maronpot RR, Grossman S, Nyska A. The prophylactic effects of natural watersoluble antioxidant from spinach and apocynin in a rabbit model of lipopolysaccharide-induced endotoxemia. Toxicol Pathol 2000; 28:588-600 PMID:10930047; http://dx.doi.org/10.1177/ 019262330002800413

53. Sebai H, Ben-Attia M, Sani M, Aouani E, GhanemBoughanmi N. Protective effect of resveratrol in endotoxemia-induced acute phase response in rats. Arch Toxicol 2009; 83:335-40; PMID:18754105; http://dx. doi.org/10.1007/s00204-008-0348-0

54. Ben-Shaul V, Sofer Y, Bergman M, Zurovsky Y, Grossman S. Lipopolysaccharide-induced oxidative stress in the liver: comparison between rat and rabbit. Shock 1999; 12:288-93; PMID:10509631; http://dx.doi.org/ 10.1097/00024382-199910000-00007

55. Lee KY, Perretta SG, Zar H, Mueller RA, Boysen PG Increase in rat plasma antioxidant activity after E. coli lipopolysaccharide administration. Yonsei Med J 2001; 42:114-9; PMID:11293489; http://dx.doi.org/ 10.3349/ymj.2001.42.1.114

56. Ivanov AI, Romanovsky AA. Prostaglandin E2 as a mediator of fever: synthesis and catabolism. Front Biosci 2004; 9:1977-93; PMID:14977603; http://dx.doi. org/10.2741/1383

57. Steiner AA, Hunter JC, Phipps SM, Nucci TB, Oliveira DL, Roberts JL, Scheck AC, Simmons DL, Romanovsky AA. Cyclooxygenase-1 or -2-which one mediates lipopolysaccharide-induced hypothermia? Am J Physiol Regul Integr Comp Physiol 2009; 297:R48594; PMID:19515980; http://dx.doi.org/10.1152/ ajpregu. 91026.2008

58. Barbieri SS, Cavalca V, Eligini S, Brambilla M, Caiani A, Tremoli E, Colli S. Apocynin prevents cyclooxygenase 2 expression in human monocytes through $\mathrm{NADPH}$ oxidase and glutathione redox-dependent mechanisms. Free Radic Biol Med 2004; 37:156-65; PMID:15203187; $\quad$ http://dx.doi.org/10.1016/j. freeradbiomed.2004.04.020

59. Egan RW, Paxton J, Kuehl FA, Jr. Mechanism for irreversible self-deactivation of prostaglandin synthetase. J Biol Chem 1976; 251:7329-35; PMID:826527

60. Kimura Y, Okuda H, Arichi S. Effects of stilbene derivatives on arachidonate metabolism in leukocytes. Biochim Biophys Acta 1985; 837:209-12; PMID:3931689; http://dx.doi.org/10.1016/0005-2760(85)90244-9

61. Okamura T, Yoshida K, Toda N. Suppression by methylene blue of prostaglandin I2 synthesis in isolated dog renal arteries. J Pharmacol Exp Ther 1990; 254:198-203; PMID:2114477

62. Salvemini D, Misko TP, Masferrer JL, Seibert K, Currie MG, Needleman P. Nitric oxide activates cyclooxygenase enzymes. Proc Natl Acad Sci U S A 1993; 90:7240-4; $\quad$ PMID:7688473; http://dx.doi.org/ 10.1073/pnas.90.15.7240

63. Paliege A, Pasumarthy A, Mizel D, Yang T, Schnermann J, Bachmann S. Effect of apocynin treatment on renal expression of COX-2, NOS1, and renin in Wistar-Kyoto and spontaneously hypertensive rats. Am J Physiol Regul Integr Comp Physiol 2006; 290:R694700; PMID:16467505; http://dx.doi.org/10.1152/ ajpregu.00219.2005

64. Mancuso C, Pistritto G, Tringali G, Grossman AB, Preziosi P, Navarra P. Evidence that carbon monoxide stimulates prostaglandin endoperoxide synthase activity in rat hypothalamic explants and in primary cultures of rat hypothalamic astrocytes. Brain Res Mol Brain Res 1997; 45:294-300; PMID:9149104; http://dx.doi.org/ 10.1016/S0169-328X(96)00258-6

65. Lee DH, Blomhoff R, Jacobs DR, Jr. Is serum gamma glutamyltransferase a marker of oxidative stress? Free Radic Res 2004; 38:535-9; PMID:15346644; http:// dx.doi.org/10.1080/10715760410001694026

66. Lee DH, Gross MD, Jacobs DR, Jr. Association of serum carotenoids and tocopherols with gamma-glutamyltransferase: the Cardiovascular Risk Developmen in Young Adults (CARDIA) Study. Clin Chem 2004; 50:582-8; PMID:14726472; http://dx.doi.org/ 10.1373/clinchem.2003.028852

67. Lim JS, Yang JH, Chun BY, Kam S, Jacobs DR, Jr., Lee $\mathrm{DH}$. Is serum gamma-glutamyltransferase inversely associated with serum antioxidants as a marker of oxidative stress? Free Radic Biol Med 2004; 37:1018-23; PMID:15336318; $\quad$ http://dx.doi.org/10.1016/j. freeradbiomed.2004.06.032

68. Lanone S, Bloc S, Foresti R, Almolki A, Taille C, Callebert J, Conti M, Goven D, Aubier M, Dureuil B, et al. Bilirubin decreases nos2 expression via inhibition of $\mathrm{NAD}(\mathrm{P}) \mathrm{H}$ oxidase: implications for protection against endotoxic shock in rats. FASEB J 2005; 19:1890-2; PMID:16129699

69. Ben-Amotz R, Bonagura J, Velayutham M, Hamlin R, Burns P, Adin C. Intraperitoneal bilirubin administration decreases infarct area in a rat coronary ischemia/ reperfusion model. Front Physiol 2014; 5:53; PMID:24600401; $\quad$ http://dx.doi.org/10.3389/ fphys.2014.00053

70. Oh SW, Lee ES, Kim S, Na KY, Chae DW, Chin HJ Bilirubin attenuates the renal tubular injury by inhibition of oxidative stress and apoptosis. BMC Nephrol
2013; 14:105; PMID:23683031; http://dx.doi.org/ 10.1186/1471-2369-14-105

71. Romanovsky AA, Szekely M. Fever and hypothermia: two adaptive thermoregulatory responses to systemic inflammation. Med Hypotheses 1998; 50:219-26; PMID:9578327; http://dx.doi.org/10.1016/S03069877(98)90022-6

72. Almeida MC, Steiner AA, Branco LG, Romanovsky AA. Neural substrate of cold-seeking behavior in endotoxin shock. PLoS One 2006; 1:e1; PMID:17183631; http://dx.doi.org/10.1371/journal.pone.0000001

73. Almeida MC, Steiner AA, Branco LG, Romanovsky AA. Cold-seeking behavior as a thermoregulatory strategy in systemic inflammation. Eur J Neurosci 2006; 23:3359-67; PMID:16820025; http://dx.doi.org/ 10.1111/j.1460-9568.2006.04854.x

74. Romanovsky AA, Shido O, Sakurada S, Sugimoto N, Nagasaka T. Endotoxin shock-associated hypothermia. How and why does it occur? Ann N Y Acad Sci 1997 813:733-7; PMID:9100963; http://dx.doi.org/ 10.1111/j.1749-6632.1997.tb51775.x

75. Liu E, Lewis K, Al-Saffar H, Krall CM, Singh A, Kulchitsky VA, Corrigan JJ, Simons CT, Petersen SR, Musteata FM, et al. Naturally occurring hypothermia is more advantageous than fever in severe forms of lipopolysaccharide- and Escherichia coli-induced systemic inflammation. Am J Physiol Regul Integr Comp Physiol 2012; 302:R1372-83; PMID:22513748; http://dx. doi.org/10.1152/ajpregu.00023.2012

76. Fujii M, Inoguchi T, Sasaki S, Maeda Y, Zheng J, Kobayashi K, Takayanagi R. Bilirubin and biliverdin protect rodents against diabetic nephropathy by downregulating $\mathrm{NAD}(\mathrm{P}) \mathrm{H}$ oxidase. Kidney Int 2010 78:905-19; PMID:20686447; http://dx.doi.org/ 10.1038/ki.2010.265

77. Kotal P, Van der Veere CN, Sinaasappel M, Elferink RO, Vitek L, Brodanova M, Jansen PL, Fevery J. Intestinal excretion of unconjugated bilirubin in man and rats with inherited unconjugated hyperbilirubinemia. Pediatr Res 1997; 42:195-200; PMID:9262222; http://dx.doi.org/10.1203/ 00006450-199708000-00011

78. Schmid R, Hammaker L. Metabolism and Disposition of C14-Bilirubin in Congenital Nonhemolytic Jaundice. J Clin Invest 1963; 42:1720-34; PMID:14083163; $\quad$ http://dx.doi.org/10.1172/ JCI104858

79. Reitman S, Frankel S. A colorimetric method for the determination of serum glutamic oxalacetic and glutamic pyruvic transaminases. Am J Clin Pathol 1957; 28:56-63; PMID:13458125

80. Szasz G. A kinetic photometric method for serum gamma-glutamyl transpeptidase. Clin Chem 1969; 15:124-36; PMID:5773262

81. Jendrassik L, Grof P. Simplified photometric methods for determination of blood-bilirubin. Biochemische Zeitschrift 1938; 297:81-9.

82. Patton CJ, Crouch SR. Spectrophotometric and kinetics investigation of Berthelot reaction for determination of ammonia. Analytical Chemistry 1977; 49:464-9; http://dx.doi.org/10.1021/ac50011a034

83. Van Pilsum JF, Martin RP, Kito E, Hess J. Determination of creatine, creatinine, arginine, guanidinoacetic acid, guanidine, and methylguanidine in biological fluids. J Biol Chem 1956; 222:225-35; PMID:13366996 


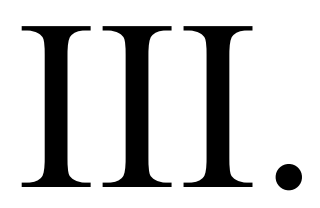




\title{
Characterization of the Thermoregulatory Response to Pituitary Adenylate Cyclase-Activating Polypeptide in Rodents
}

\author{
Eszter Banki • Eszter Pakai • Balazs Gaszner • Csaba Zsiboras • \\ Andras Czett • Paras Rahul Parkash Bhuddi • Hitoshi Hashimoto • \\ Gabor Toth • Andrea Tamas • Dora Reglodi • Andras Garami
}

Received: 15 April 2014 / Accepted: 23 June 2014 / Published online: 4 July 2014

(C) Springer Science+Business Media New York 2014

\begin{abstract}
Administration of the long form (38 amino acids) of pituitary adenylate cyclase-activating polypeptide (PACAP38) into the central nervous system causes hyperthermia, suggesting that PACAP38 plays a role in the regulation of deep body temperature $\left(T_{\mathrm{b}}\right)$. In this study, we investigated the thermoregulatory role of PACAP38 in details. First, we infused PACAP38 intracerebroventricularly to rats and measured their $T_{\mathrm{b}}$ and autonomic thermoeffector responses. We found that central PACAP38 infusion caused dose-dependent hyperthermia, which was brought about by increased thermogenesis and tail skin vasoconstriction. Compared to intracerebroventricular administration, systemic (intravenous) infusion of the same dose of PACAP38 caused significantly smaller hyperthermia, indicating a central site of action. We then investigated the thermoregulatory phenotype of mice lacking the Pacap gene $\left(\right.$ Pacap $\left.^{-1}\right)$. Freely moving Pacap ${ }^{-1-}$ mice had higher locomotor activity throughout the day and elevated deep $T_{\mathrm{b}}$ during the light phase. When the Pacap $^{-/}$ mice were loosely restrained, their metabolic rate and $T_{\mathrm{b}}$ were lower compared to their wild-type littermates. We conclude
\end{abstract}

Eszter Banki and Eszter Pakai contributed equally to this work.

E. Banki · B. Gaszner · A. Czett · A. Tamas · D. Reglodi Department of Anatomy PTE-MTA "Lendulet" PACAP Research

Team, Medical School, University of Pecs, Pecs, Hungary

E. Pakai $\cdot$ C. Zsiboras $•$ P. R. P. Bhuddi $\cdot$ A. Garami $(\bowtie)$

Department of Pathophysiology and Gerontology, Medical School,

University of Pecs, 12 Szigeti Str, Pecs, Hungary H7624

e-mail: andras.garami@aok.pte.hu

H. Hashimoto

Graduate School of Pharmacological Sciences, Osaka University,

Osaka, Japan

G. Toth

Department of Medical Chemistry, Faculty of Medicine, University

of Szeged, Szeged, Hungary that PACAP38 causes hyperthermia via activation of the autonomic cold-defense thermoeffectors through central targets. Pacap $^{-/-}$mice express hyperkinesis, which is presumably a compensatory mechanism, because under restrained conditions, these mice are hypometabolic and hypothermic compared to controls.

Keywords PACAP $\cdot$ Hyperthermia $\cdot$ Thermoregulation . Locomotor activity $\cdot$ Autonomic thermoeffectors

\section{Introduction}

The long form of the pituitary adenylate cyclase-activating polypeptide (PACAP) consists of 38 amino acids (PACAP38), and together with its receptors (PAC1 and VPAC1/2 receptors), it is widely expressed both in peripheral organs and in the central nervous system (CNS), explaining its diverse biological functions (for review, see Vaudry et al. 2009). A shorter form of the peptide (PACAP27) has also been identified (Miyata et al. 1990), but since in most tissues PACAP38 is the predominant form with a concentration ratio of PACAP27:PACAP38 to $<1: 9$ (Vaudry et al. 2000, 2009) and because the effects of the PACAP27 and PACAP38 on cAMP formation (Nowak and Kuba 2002), vascular responses (Lenti et al. 2007), and on body temperature (Seeliger et al. 2010) are similar, the current study focused on the effects of PACAP38. In the CNS, PACAP exerts neurotrophic effects (Vaudry et al. 1999; Njaine et al. 2014) as well as neuroprotective actions in experimental models of ischemia (Reglodi et al. 2000; Danyadi et al. 2014), Parkinson's disease (Brown et al. 2013, 2014) and viral neurotoxicity (Rozzi et al. 2014). The peptide is known to regulate pituitary hormone secretion (Koves et al. 2014) and improve barrier properties of the endothelial cells in the brain (Wilhelm et al. 2014). On the periphery, PACAP has been shown to have an anti- 
inflammatory role in diabetic kidney damage (Banki et al. 2013, 2014), neurogenic inflammation (Helyes et al. 2007), and contact dermatitis (Kemeny et al. 2010). PACAP has been reported to play an important role in the regulation of numerous homeostatic processes by influencing circadian rhythm (Nagy and Csernus 2007; Racz et al. 2008) and food intake (Hawke et al. 2009; Resch et al. 2011). Since the isolation of PACAP38 by Miyata et al. (1989), a large number of studies have been conducted to identify the role of the peptide in various homeostatic functions, including the regulation of deep body temperature $\left(T_{\mathrm{b}}\right)$.

Supporting the role of PACAP38 in thermoregulation, the peptide and its receptors are broadly expressed in main thermoregulatory areas of the brain, including the lateral parabrachial area, the preoptic area of the hypothalamus (POA), the dorsomedial nucleus of the hypothalamus, the periaqueductal gray matter, and the nucleus raphe pallidus (Palkovits et al. 1995; Joo et al. 2004; Das et al. 2007). In physiological studies, the injection of PACAP38 into the lateral cerebral ventricle (Pataki et al. 2000, 2003; Hawke et al. 2009), the intrathecal space (Inglott et al. 2011), or into the ventromedial hypothalamic nucleus (Resch et al. 2011, 2013) caused an increase of $T_{\mathrm{b}}$ in rats and mice. Although elevation of non-shivering thermogenesis (Hawke et al. 2009; Inglott et al. 2011; Resch et al. 2011) and increase of locomotor activity (Resch et al. 2011, 2013) have been shown to contribute to the PACAP38-induced hyperthermia, no study has yet been conducted to investigate simultaneous activation of autonomic thermoeffectors.

The thermoregulatory system operates as a federation of independent thermoeffector loops, in which each loop consists of a sensor, an afferent, and an efferent branch (Romanovsky 2007a). For example, environmental cold activates cutaneous cold receptors and signals from the skin are conveyed to thermoregulatory centers in the brain from where autonomic (thermogenesis, cutaneous vasonconstriction) and behavioral (warmth seeking) cold-defense effectors are driven (Romanovsky 2014). These defense mechanisms can be modulated from peripheral (Almeida et al. 2012) as well as from central sites (Nakamura and Morrison 2008b). A substance such as PACAP38 can act at any element of a thermoeffector loop and cause the same effect: hyperthermia. Since the thermoregulatory response to PACAP38 has not been compared between systemic (outside the blood-brain barrier) and central (into CNS) substance delivery, it cannot be firmly stated whether the primary site of action of PACAP38 is on peripheral afferents, in the brain as proposed by Resch et al. (2011, 2013), in the spinal cord as suggested by Inglott et al. (2011), or on the efferent neural pathway of a thermoeffector (e.g., that of the brown adipose tissue).

In addition to exogenous PACAP38 administration, genetically modified mice lacking the Pacap gene have also been utilized to investigate how the absence of PACAP affects deep
$T_{\mathrm{b}}$. These studies obtained contradictory results showing that the absence of PACAP in mice lead to increased (Hashimoto et al. 2001) versus unchanged locomotor activity (Adams et al. 2008), as well as to lower (Hashimoto et al. 2009) versus unchanged $T_{\mathrm{b}}$ as compared to controls (Cummings et al. 2008).

In the present study, we characterized the dose-dependency of and the autonomic thermoeffector pattern involved in the response to PACAP38. Then, in a comparative experiment, we addressed the question whether exogenous PACAP38 administration acts primarily through peripheral or central targets in rats. Lastly, as an additional approach to identify the role of PACAP in thermoregulation, we used mice lacking the Pacap gene and studied how the absence of PACAP affects circadian changes of their deep $T_{\mathrm{b}}$ and locomotor activity as well as their basal $T_{\mathrm{b}}$ and metabolic rate.

\section{Materials and Methods}

\section{Animals}

The physiological experiments were performed in 40 adult male Wistar rats and 42 adult mice of both sexes. The mice had the Pacap gene homozygously either present $\left(\right.$ Pacap $\left.^{+/+}\right)$or absent $\left(\right.$ Pacap $^{-1}$ ) due to a targeted disruption (Hashimoto et al. 2001). Generation by a gene-targeting technique, maintenance, and backcrossing of Pacap ${ }^{-/-}$mice on a CD1 background has been reported previously (Hashimoto et al. 2001, 2009). Animals were housed in temperature-controlled rooms on a $12 \mathrm{~h}$ light-dark cycle. Standard rodent chow and tap water were available ad libitum. At the time of the experiments, the rats weighed $331 \pm 33 \mathrm{~g}$ and the mice weighed $24 \pm 2 \mathrm{~g}$.

Rats and mice were extensively handled and then habituated to staying inside wire-mesh cylindrical confiners. The cylindrical confiner prevented the animal from turning around, but allowed for some back-and-forth movements; it was used in the respirometry setup (see the "Experimental Setups" section below).

All procedures were conducted under protocols approved by Institutional Animal Use and Care Committee of the University of Pecs and were in accordance with the directives of the National Ethical Council for Animal Research and those of the European Communities Council (86/609/EEC).

\section{Surgeries}

Mice

Mice were anesthetized with a ketamine-xylazine cocktail $(81.7$ and $9.3 \mathrm{mg} / \mathrm{kg}$, respectively, i.p.) and received antibiotic protection (gentamycin, $6 \mathrm{mg} / \mathrm{kg}$, i.m.). During surgery, a mouse was heated with a temperature-controlled heating pad 
(model TMP-5a; Supertech Instruments UK Ltd., London, UK) placed under a surgery board.

A mouse designated for an experiment in the telemetry setup was implanted with a miniature telemetry transmitter (G2 E-Mitter series; Mini Mitter, Bend, OR, USA) to record abdominal temperature ( $T_{\mathrm{ab}}$, a measure of deep $\left.T_{\mathrm{b}}\right)$ and locomotor activity. The device was inserted into the peritoneal cavity via midline laparotomy and fixed to the lateral abdominal wall (right side) with a suture. The surgical wound was sutured in layers. After the surgery, mice were allowed to fully recover for 10 days before data collection started.

\section{Rats}

Surgeries were performed under ketamine-xylazine (55.6 and $5.5 \mathrm{mg} / \mathrm{kg}$, respectively, i.p.) anesthesia and antibiotic protection (gentamycin, $6 \mathrm{mg} / \mathrm{kg}$, i.m.). Experiments were performed 2 to 4 days after surgery. Each rat was implanted with either an intravenous (i.v.) catheter or an intracerebroventricular (i.c.v.) cannula as described below.

For i.v. catheter implantation, a small longitudinal incision was made on the ventral surface of the neck, left of the trachea. The left jugular vein was exposed, freed from its surrounding connective tissue, and ligated. A silicone catheter (ID $0.5 \mathrm{~mm}$, OD $0.9 \mathrm{~mm})$ filled with heparinized $(10 \mathrm{U} / \mathrm{ml})$ saline was passed into the superior vena cava through the jugular vein and secured in place with ligatures. The free end of the catheter was knotted, tunneled under the skin to the nape, and exteriorized. The wound was sutured. The catheter was flushed with heparinized saline $(10 \mathrm{U} / \mathrm{ml})$ on the day after the surgery and every other day. This technique was repeatedly used in our earlier studies (Petervari et al. 2005; Garami et al. 2010).

For i.c.v. cannulation, each rat was fixed to a stereotaxic apparatus as carried out in our earlier studies (Petervari et al. 2009, 2010). The scalp was incised over the sagittal suture; the periosteum was excised; the skull was cleaned and dried; two supporting microscrews were driven into the skull; and a small hole was drilled in the skull $1.0 \mathrm{~mm}$ antero-posterior from bregma and $1.5 \mathrm{~mm}$ lateral from midline. A 22-G steel guide cannula was attached to a plastic tube fitted into a stereotaxic manipulator (Narishige Scientific Instruments Laboratory, Tokyo, Japan), which was used to insert the cannula into the brain through the bone hole. The tip of the cannula was placed within the right lateral ventricle $(3.8 \mathrm{~mm}$ from dura). The cannula was secured to the supporting microscrews with dental cement and released from the manipulator. The guide cannula was closed by a dummy cannula.

\section{Experimental Setups}

Physiological experiments in unanesthetized animals were conducted in either the respirometry setup or the telemetry setup. The respirometry setup was used (a) to measure the thermoregulatory responses of rats to non-stressful administration of PACAP38 and (b) to assess the basal thermoregulatory parameters of untreated, loosely restrained Pacap $^{-1-}$ and Pacap $^{+/+}$mice. The telemetry setup was used only in untreated, freely-moving $\mathrm{Pacap}^{-/-}$and $\mathrm{PaCap}^{+/+}$mice to record their $T_{\mathrm{ab}}$ and locomotor activity over a longer period (24 h) of time.

In the respirometry setup, a rat or mouse equipped with copper-constantan thermocouples (Omega Engineering, Stamford, CT, USA) to measure colonic $\left(T_{\mathrm{c}}\right)$, and tail skin temperature $\left(T_{\mathrm{sk}}\right)$ was placed in a confiner. The colonic thermocouple was inserted 10 or $3 \mathrm{~cm}$ beyond the anal sphincter in rats and mice, respectively, and fixed to the base of the tail with a loop of adhesive tape. The skin thermocouple was positioned on the lateral surface of the tail (at the boundary of the proximal and middle thirds) and insulated from the environment with tape. The thermocouples were plugged into a data logger (Cole-Parmer, Vernon Hills, IL, USA). Then, each animal in its confiner was transferred to a Plexiglas chamber of the four-chamber open-circuit calorimeter integrated system (Oxymax Equal Flow, Columbus Instruments, Columbus, OH, USA). The chamber was sealed, submerged into a temperature-controlled water bath, and continuously ventilated with room air $(1,000$ and $200 \mathrm{ml} / \mathrm{min}$ for rats and mice, respectively). The fractional concentration of oxygen was measured in the air entering and exiting the chamber, and the rate of oxygen consumption $\left(\mathrm{VO}_{2}\right)$ was calculated according to the manufacturer's instructions using the Oxymax Windows software (v3.1). When present, the venous catheter was connected to a polyethylene-50 extension filled with the drug of interest. When the animal had an i.c.v. cannula, a needle injector was fitted into the guide cannula and connected to a polyethylene extension (ID $0.28 \mathrm{~mm}$, OD $0.61 \mathrm{~mm}$ ). The extension was passed through a port of the chamber and connected to a syringe. All experiments were conducted at an ambient temperature $\left(T_{\mathrm{a}}\right)$ of $28.0^{\circ} \mathrm{C}$ or $31.0^{\circ} \mathrm{C}$, which is thermoneutral for rats and mice, respectively, in this setup (Balasko et al. 2010; de Oliveira et al. 2014).

In the telemetry setup, mice were studied inside their home cages. Telemetry receivers (model ER-4000; Mini Mitter) were positioned in a temperature-controlled room, and the home cages of mice were placed on top of the receivers. In this setup, a $T_{\mathrm{a}}$ of $27.0^{\circ} \mathrm{C}$ was used, which is near the lower end of the thermoneutral zone for mice (Kanizsai et al. 2009). The mouse was preimplanted with a telemetry transmitter to measure $T_{\mathrm{ab}}$ and locomotor activity. The latter has been shown to play an important thermoregulatory role in small rodents such as rats and mice (Mount and Willmott 1967; Brown et al. 1991; Weinert and Waterhouse 1998). A similar method was also used to detect small differences in the thermoregulatory phenotype between transient receptor potential vanilloid-1 (TRPV1) channel knockout and control mice (Kanizsai et al. 2009; Garami et al. 2011). 


\section{Substance Administration}

PACAP38 was synthesized at the University of Szeged as described in details elsewhere (Gasz et al. 2006). Lyophilized aliquots of PACAP38 were stored at $4{ }^{\circ} \mathrm{C}$. On the day of the experiment, an aliquot was dissolved in saline to give a working solution of PACAP38 at $0.3,0.6$, or $6 \mathrm{mg} / \mathrm{ml}$. For the i.v. drug administration, the $0.3 \mathrm{mg} / \mathrm{ml}$ working solution was infused to rats at a rate of $87 \mu \mathrm{l} / \mathrm{min} / \mathrm{kg}(\sim 29 \mu \mathrm{l} / \mathrm{min} / \mathrm{rat})$ for $4 \mathrm{~min}$ to deliver a final dose of PACAP38 at $100 \mu \mathrm{g} / \mathrm{kg}$ $(\sim 33 \mu \mathrm{g} / \mathrm{rat})$. For i.c.v. drug administration, $5 \mu \mathrm{l}$ of the 0.6 or $6 \mathrm{mg} / \mathrm{ml}$ working solutions were infused over a 3-min time period to deliver PACAP38 at doses of 10 and $100 \mu \mathrm{g} / \mathrm{kg}$ $(\sim 3.3$ and $33 \mu \mathrm{g} / \mathrm{rat})$, respectively. Control animals were infused with saline.

\section{Immunocytochemistry for c-Fos}

The labeling was performed as published earlier (Gaszner et al. 2012). Briefly, Pacap ${ }^{-/}$and Pacap ${ }^{+/+}$ mice were injected within a time period of 2 min with i.p. administered Nembutal (sodium-pentobarbital; $100 \mathrm{mg} / \mathrm{kg}$ body weight; Sanofi, Budapest, Hungary). All mice became unconscious within $2 \mathrm{~min}$. Then, they were transcardially perfused with $25 \mathrm{ml}$ of $0.1 \mathrm{M}$ sodium phosphate-buffered saline (PBS; pH 7.4) for $2 \mathrm{~min}$, followed by perfusion with $150 \mathrm{ml}$ of ice-cold $4 \%$ paraformaldehyde in $0.2 \mathrm{M}$ Millonig sodium phosphate buffer ( $\mathrm{pH} 7.4$ ), for $20 \mathrm{~min}$. Brains were removed and post-fixed for $24 \mathrm{~h}$. Coronal sections $(30 \mu \mathrm{m})$ were prepared on vibratome (Lancer, Ted Pella Inc., Redding, CA, USA) and stored in anti-freeze solution at $-20{ }^{\circ} \mathrm{C}$. For free-floating diaminobenzidine (DAB; Sigma Chemical, Zwijndrecht, The Netherlands) immunocytochemistry, sections were washed $6 \times 10 \mathrm{~min}$ in $0.1 \mathrm{M}$ PBS. Consecutively, sections were incubated in $0.5 \%$ Triton X-100 (Sigma Chemical), then in a blocking buffer of $2 \%$ normal goat serum (NGS, Jackson Immunoresearch Europe Ltd., Suffolk, UK) in PBS for $30 \mathrm{~min}$. Sections were transferred into an antiserum solution (1:500) raised against c-Fos (Santa Cruz Biotechnology Inc., sc-52, Santa Cruz, CA, USA). After $3 \times 10$ min washes, sections were treated with biotinylated goat anti-rabbit $\mathrm{IgG},(1: 200)$ containing $2 \% \mathrm{NGS}$, for $2 \mathrm{~h}$ at $20{ }^{\circ} \mathrm{C}$. After a rinse in cold PBS, sections were placed into avidin-biotin-complex solution (Vectastain Elite ABC Kit, Vector Laboratories, Burlingame, CA, USA), for $1 \mathrm{~h}$ at $20^{\circ} \mathrm{C}$, followed by PBS for $3 \times 10 \mathrm{~min}$ washes. The immunoreaction was visualized using $0.02 \% \mathrm{DAB}$ in Tris buffer with $0.003 \% \mathrm{H}_{2} \mathrm{O}_{2}$, for $10 \mathrm{~min}$. The reaction was observed under microscope and stopped with PBS. After washes, sections were mounted on gelatin-coated slides, dried, cleared by $2 \times 10 \mathrm{~min}$ xylene treatment, coverslipped with DePex (Fluka, Heidelberg, Germany), and studied with a Nikon Microphot FXA microscope and Spot RT color digital camera (Nikon, Tokyo, Japan).

The polyclonal c-Fos antiserum (Santa Cruz Biotechnology Inc., sc-52, Santa Cruz, CA, USA) had been generated against the 3-16 amino acid c-Fos peptide fragment of human origin. Preadsorption with $0.1,1$, and $10 \mu \mathrm{g}$ synthetic c-Fos blocking peptide (Santa Cruz Biotechnology Inc., sc-52 P, Santa Cruz, CA, USA); moreover, omission or replacement of the c-Fos serum by nonimmune rabbit serum effectively prevented the staining. The cross reactivity of this antiserum with other Fosrelated proteins was excluded earlier by Ryabinin et al. (1999). Western blot analysis support the specificity of the antibody used (for details see supplier's web site: http://datasheets.scbt.com/sc-52.pdf).

Microscopy, Digital Imaging, and Morphometry

Per animal, the cell counts positive for c-Fos were determined in five serial sections, each interspaced by $60 \mu \mathrm{m}$ in the median preoptic nucleus (MnPO) and medial preoptic area (MPO) according to Paxinos and Franklin (2004) atlas. Cell counting was carried out on non-edited digital images using ImageJ software (version 1.37, NIH, Bethesda, MD, USA). Quantitation was performed in a double-blind setup by a colleague who is an expert in the rodent neuroanatomy, but was blinded to the identity of preparations. Two representative digital images were grayscaled and contrasted using Photoshop software (Adobe, San Jose, CA, USA) for publication purposes.

\section{Data Processing and Analysis}

Data on $T_{\mathrm{c}}, T_{\mathrm{ab}}$, heat loss index $(H L I)$, and $V \mathrm{O}_{2}$ were compared by two-way ANOVA followed by Fisher's LSD post hoc tests, as appropriate. The $H L I$ was calculated as:

$H L I=\frac{\mathrm{T}_{\mathrm{sk}}-T_{a}}{T_{c}-T_{a}}$.

The $H L I$ changes between 0 (maximum heat conservation due to skin vasoconstriction) and 1 (theoretical maximum heat loss due to skin vasodilation; Romanovsky et al. 2002). Numbers of the c-Fos-positive cells were compared by Student's two sample $t$ test (alpha $=5 \%$ ). For statistical analysis, Sigmaplot 11.0 (Systat Software, San Jose, CA, USA) software was used. All data are reported as mean \pm SE. 


\section{Results}

Characteristics of the Thermoregulatory Response to Central (i.c.v.) PACAP38 Administration

To characterize the thermoregulatory effect of PACAP38 in details, we infused 10 or $100 \mu \mathrm{g} / \mathrm{kg}$ of the peptide (or saline) into the lateral cerebral ventricle of rats and recorded their $T_{\mathrm{c}}$, $T_{\mathrm{sk}}$, and $\mathrm{VO}_{2}$ in the respirometry setup. In all rats studied, infusion of saline did not have any influence on $T_{\mathrm{c}}, H L I$, and $\mathrm{VO}_{2}$ (Fig. 1). On the contrary, both of the applied doses of PACAP38 caused a marked rise in the $T_{\mathrm{c}}$ starting already at $10 \mathrm{~min}$ after the injection $(p<0.001$ for both; Fig. 1). The magnitude of the PACAP38-induced hyperthermia was dosedependent with a maximal $T_{\mathrm{c}}$ change of $2.0 \pm 0.3{ }^{\circ} \mathrm{C}$ and $1.4 \pm$ $0.3^{\circ} \mathrm{C}$ at the dose of 100 and $10 \mu \mathrm{g} / \mathrm{kg}$, respectively $(p<0.001$ for both). Statistical analysis also revealed significant

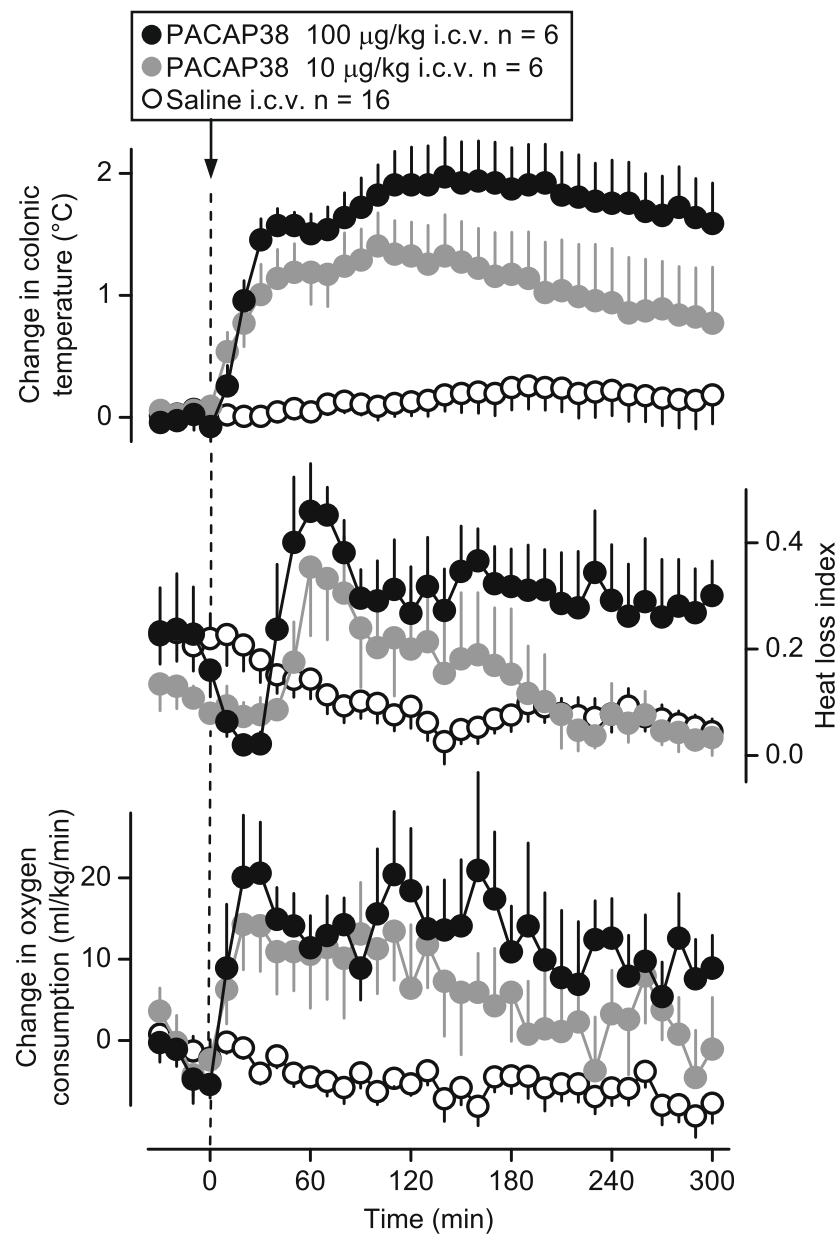

Fig. 1 Thermoeffector and $T_{\mathrm{c}}$ responses of rats to different doses (10 and $100 \mu \mathrm{g} / \mathrm{kg}$ ) of PACAP38 or saline administered i.c.v. The changes of $T_{\mathrm{c}}$ (a measure of deep $T_{\mathrm{b}}$ ) are shown in the upper panel; alterations in the activity of the two main autonomic thermoeffectors, $\mathrm{HLI}$ and $\mathrm{VO}_{2}$, are depicted in the middle and lower panel, respectively. These experiments were performed in the respirometry setup at a $T_{\mathrm{a}}$ of $28{ }^{\circ} \mathrm{C}$. Numbers of animals in the corresponding groups are indicated in the figure difference $(p<0.001)$ between the effects of the $10 \mathrm{vs}$. $100 \mu \mathrm{g} / \mathrm{kg}$ dose of PACAP38 on $T_{\mathrm{c}}$. The hyperthermic response to intrabrain administration of PACAP38 is in harmony with earlier reports on the effect of PACAP38 injection on $T_{\mathrm{b}}$ in rats (Pataki et al. 2000, 2003; Resch et al. 2011, 2013).

In the case of the $100 \mu \mathrm{g} / \mathrm{kg}$ dose, the development of the hyperthermia was preceded by significant tail skin vasoconstriction (as indicated by a decreased $H L I ; p<0.05$ ). This is a novel finding of our study, and to our knowledge, the first to report cutaneous vasomotor responses to i.c.v. PACAP38 in conscious rats. It indicates that the thermoregulatory (constrictor) effect of PACAP38 on the cutaneous vascular tone is different from its direct (dilator) effect on skin vessels, which was shown earlier in small rodents (Absood et al. 1992; Tsueshita et al. 2002). The initial drop of HLI lasted for $\sim 40 \mathrm{~min}$, and then, it was followed by a pronounced elevation of $H L I$ due to tail skin vasodilation, which remained significantly $(p<0.05)$ higher than the HLI of saline-treated rats until the end of the experiment in accordance with the vasodilatory effect of PACAP38 reported earlier (Absood et al. 1992; Tsueshita et al. 2002). Rats treated with $10 \mu \mathrm{g} / \mathrm{kg}$ PACAP38 i.c.v. had low $H L I$ already before substance administration; thus, the initial drop of $H L I$ in this group could not be observed, however, $\sim 50 \mathrm{~min}$ after drug infusion $H L I$ increased above baseline levels and became higher than that of controls $(p<0.05)$, although the magnitude and the duration of $H L I$ elevation were smaller than those observed at $100 \mu \mathrm{g} / \mathrm{kg}$.

Similarly to $T_{\mathrm{c}}$, the $\mathrm{VO}_{2}$ of the rats increased already at 10 min after i.c.v. PACAP38 administration as compared to saline-treated animals in a dose-dependent manner. It reached a maximal rise of $21 \pm 6$ and $14 \pm 6 \mathrm{ml} / \mathrm{kg} / \mathrm{min}$ at 100 and $10 \mu \mathrm{g} / \mathrm{kg}$, respectively ( $p<0.001$ for both). This finding is in harmony with previous studies, in which PACAP38 elevated the metabolic rate (Hawke et al. 2009; Inglott et al. 2011; Resch et al. 2011). In addition, our results demonstrate that simultaneous immediate activation of both autonomic colddefense thermoeffectors (cutaneous vasoconstriction and brown adipose tissue thermogenesis) contribute to the development of hyperthermia in response to PACAP38.

Investigation of the Thermoregulatory Response to Systemic (i.v.) PACAP38 Administration

The dose-dependent hyperthermia in response to PACAP38 injection either into the lateral ventricle (current study; Pataki et al. 2000, 2003) or into the hypothalamus (Resch et al. 2011, 2013) suggests that the site of action for PACAP38 is located in the CNS, but one can not rule out the possibility that intrabrain PACAP38 administration acts on central elements of a thermoregulatory loop, which receives its afferentation from the periphery and through central neural structures innervates the corresponding thermoeffector. Such scenario is plausible based on the modern concept of thermoregulation, 
according to which deep $T_{\mathrm{b}}$ is controlled by a federation of independent loops of thermoeffectors (for review, see Romanovsky 2007b). In such a loop, activation of the afferent pathway upstream from the central nuclei can evoke equal effects as its activation at any, for example central, part of the loop.

To study the possibility of a peripheral site of action for PACAP38 in a comparative experiment, we investigated whether the same $(100 \mu \mathrm{g} / \mathrm{kg})$ dose of PACAP38 that caused pronounced hyperthermia when injected i.c.v. has a similar effect on deep $T_{\mathrm{b}}$ in the case of a systemic (i.v.) administration. Infusion of saline did not have thermoregulatory effects in the rats studied (Fig. 2). When PACAP38 at $100 \mu \mathrm{g} / \mathrm{kg}$ was infused i.v., it caused a slight, but significant $(p<0.05)$ rise of $T_{\mathrm{c}}$. Both the maximum $T_{\mathrm{c}}$ elevation $\left(\sim 0.3{ }^{\circ} \mathrm{C}\right)$ and the duration $(60 \mathrm{~min})$ of the hyperthermic response to systemic PACAP38 infusion were markedly less than what i.c.v. administration of the same dose evoked $(p<0.05)$. One can argue that neurons in the CNS were exposed to higher local concentrations of PACAP38 than neurons on the periphery after infusion of the same dose into the two compartments, but the differences in local concentrations are unlikely to account

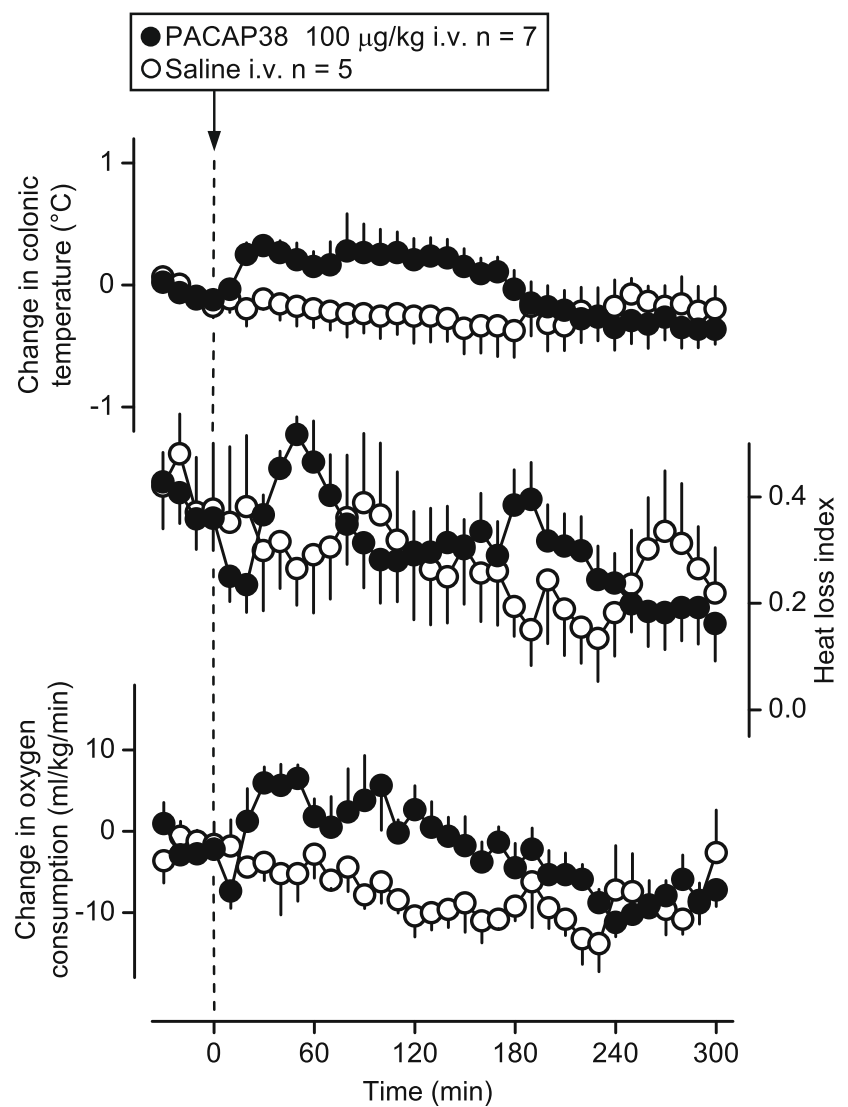

Fig. 2 The thermoregulatory response of rats to PACAP38 $(100 \mu \mathrm{g} / \mathrm{kg})$ or saline administered i.v. Changes of $T_{\mathrm{c}}$ (upper panel), HLI (middle panel), and $\mathrm{VO}_{2}$ (bottom panel) are shown with the same scale intervals as in Fig. 1. The experimental conditions were also identical to those described in Fig. 1 (respirometry setup, $T_{\mathrm{a}}$ of $28^{\circ} \mathrm{C}$ ). Numbers of animals in the corresponding groups are indicated in the figure for the substantial differences observed in the $T_{\mathrm{c}}$ response. Importantly, the response to PACAP38 at $100 \mu \mathrm{g} / \mathrm{kg}$ i.v. was substantially smaller both in magnitude ( $\sim 5$-fold lesser) and in duration ( $\sim 2$ times shorter) than the effect of a tenfold smaller dose $(10 \mu \mathrm{g} / \mathrm{kg})$ delivered i.c.v. (Fig. 1). Similarly to what we observed after i.c.v. drug delivery, in the case of the i.v. infusion of PACAP38, the hyperthermia was also brought about by a decreased heat loss and an increased $\mathrm{VO}_{2}$, although activity of both thermoeffectors changed to a much lesser extent than after i.c.v. delivery (Fig. 2). The result that even a tenfold lower dose of PACAP38 caused much stronger hyperthermia after i.c.v. administration compared to i.v. delivery, unequivocally shows that the site of action for the thermoregulatory response to PACAP38 is situated within the CNS.

\section{Thermoregulatory Characteristics of $\mathrm{Pacap}^{-/-}$Mice}

After we characterized the thermoregulatory response to exogenous PACAP38 administration, we wanted to know how the absence of PACAP affects deep $T_{\mathrm{b}}$.

First, we studied the circadian changes of $T_{\mathrm{ab}}$ and locomotor activity in freely moving Pacap $^{-/-}$and Pacap $^{+/+}$mice (Fig. 3a). Representing the characteristic circadian rhythm of rodents, mice of both genotypes had lower $T_{\mathrm{ab}}$ and activity levels during the light (inactive) phase than during the dark (active) phase. In accordance with the study by Hashimoto et al. (2001), we found that Pacap $^{-/-}$mice were more active than their wild-type littermates during both the light and the dark phase of the day $(p<0.001)$. During most of the light phase (between 5 a.m. and 3 p.m.), the increased locomotor activity resulted in a moderately higher $T_{\mathrm{ab}}$ in the Pacap $^{-/}$ mice compared to controls $(p<0.05)$; but in the night, there was no significant difference in $T_{\mathrm{ab}}$ between the genotypes (Fig. 3a). Similar results on the effect of hyperactivity on deep $T_{\mathrm{b}}$ were also demonstrated in chicken (Aschoff and von SaintPaul 1973) and in mice (Weinert and Waterhouse 1998, 1999), showing that elevated locomotor activity resulted in higher $T_{\mathrm{b}}$ during the inactive phase, but not during the active phase. It can be assumed that the different light-dark influence of locomotor activity on deep $T_{\mathrm{b}}$ can originate from the circadian changes of cutaneous vasodilation, thus heat loss mechanisms (Weinert and Waterhouse 1998).

Next, we measured the basal $T_{\mathrm{c}}$ and $V \mathrm{VO}_{2}$ in loosely restrained Pacap ${ }^{-/-}$and Pacap ${ }^{+/+}$mice (Fig. 3b). These experiments were performed in the respirometry setup (see the "Materials and Methods" section), so we could minimize the influence of locomotor activity on $T_{\mathrm{c}}$ and $\mathrm{VO}_{2}$. We recorded the basal thermoregulatory parameters for $60 \mathrm{~min}$ starting from 11 a.m. because this time period corresponded to the biggest difference in deep $T_{\mathrm{b}}$ between freely moving $\mathrm{Pacap}^{-/}$ and $\mathrm{PaCap}^{+/+}$mice (Fig. 3a). As shown in Fig. 3b, the basal $\mathrm{VO}_{2}$ was significantly lower in $\mathrm{Pacap}^{-/-}$mice as compared to 


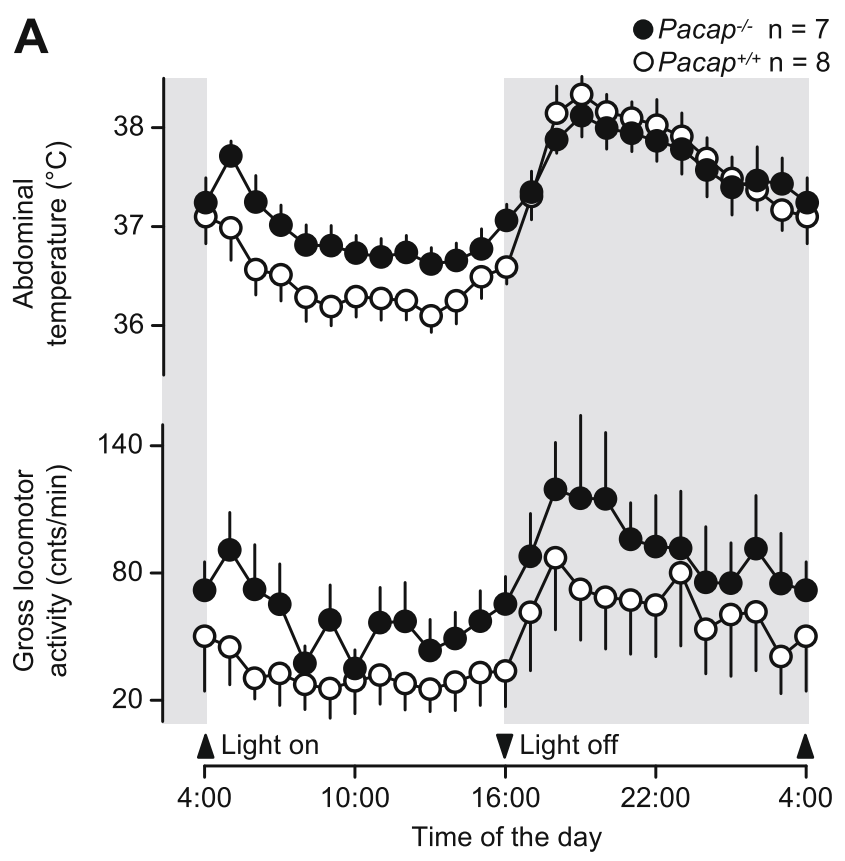

B
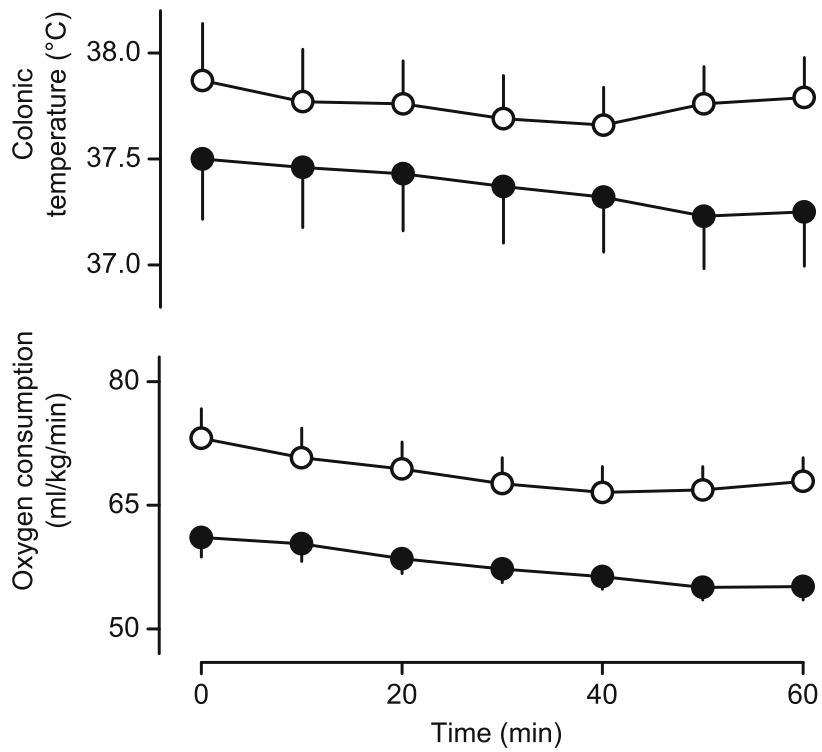

Fig. 3 The thermoregulatory phenotype of $\mathrm{Pacap}^{-/-}$and $\mathrm{Pacap}^{+/+}$mice. a Circadian changes of $T_{\mathrm{ab}}$ and locomotor activity in freely moving Pacap $^{-/}$and Pacap ${ }^{+/+}$mice. These experiments were performed in the telemetry setup at a $T_{\mathrm{a}}$ of $27^{\circ} \mathrm{C}$. b Basal $T_{\mathrm{c}}$ and $\mathrm{VO}_{2}$ of loosely restrained Pacap $^{-/-}$and Pacap ${ }^{+/+}$mice. Recordings of $T_{\mathrm{c}}$ and $\mathrm{VO}_{2}$ were performed between $11 \mathrm{a} . \mathrm{m}$. and $12 \mathrm{p} . \mathrm{m}$. in the respirometry setup at a $T_{\mathrm{a}}$ of $31^{\circ} \mathrm{C}$. Numbers of animals in the corresponding groups are indicated in the figure

controls throughout the experiment $(p<0.001)$. As a consequence of their hypometabolism, $T_{\mathrm{c}}$ of the Pacap $^{-/-}$mice was also slightly lower than that of controls $(p<0.01)$.

To assess which neurons are responsible for maintaining the reduced resting metabolic rate in Pacap $^{-/-}$mice, we measured expression of the inducible transcription factor cFos, a marker of neuronal activation (Sagar et al. 1988), in the MnPO and MPO (Fig. 4a). It is well established that neurons in these brain areas are involved in the regulation of thermogenesis (Nakamura and Morrison 2008b; Romanovsky et al. 2009). In the MnPO, we found no statistical difference in the number of c-Fos-positive cells between $\mathrm{Pacap}^{-/-}$and Pacap $^{+/+}$mice; however, c-Fos expression was nearly three times higher $(p<0.05)$ in the MPO of the Pacap $^{-/-}$mice as compared to their wild-type littermates (Fig. 4b). It has been shown that GABAergic neurons in the MPO tonically suppress BAT thermogenesis (Osaka 2004) and can be regarded as the first effector neurons of the thermoregulatory loops controlling autonomic thermoeffectors (Romanovsky et al. 2009), therefore, our current findings in Pacap $^{-/}$mice suggest that the absence of PACAP results in an increased activation of the inhibitory MPO neurons leading to more pronounced suppression of thermogenesis.

\section{Discussion}

Our findings clearly demonstrate that central, rather than peripheral mechanisms are involved in the hyperthermia-

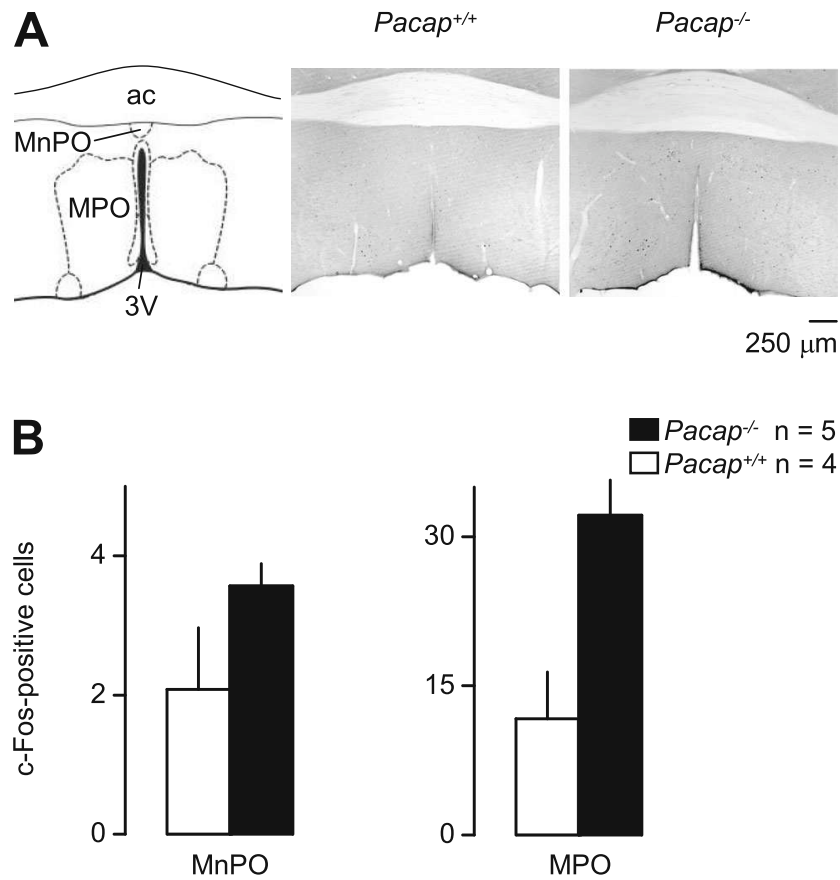

Fig. 4 The expression of c-Fos positive cells in the MnPO and MPO of Pacap $^{-/-}$and Pacap $^{+/+}$mice. a Schematic drawing from Paxinos and Franklin (2004) atlas and representative photomicrographs of coronal sections from the $\mathrm{MnPO}$ and $\mathrm{MPO}$ at the anterior-posterior coordinate of $0.14 \mathrm{~mm}$ from Bregma. The anterior commissure (ac) and the third ventricle $(3 \mathrm{~V})$ are shown as landmarks. b Quantitative analyses of c-Fos immunoreactive cells in the MnPO and MPO. Numbers of animals in the corresponding groups are indicated in the figure 
inducing effect of PACAP38. Although PACAP38-induced hyperthermia has been studied earlier in small rodents, based on data currently available from those experiments, no firm conclusion could be drawn about the site of the thermoregulatory action of PACAP38. In previous studies, PACAP38 was administered only into the CNS (Pataki et al. 2000, 2003; Hawke et al. 2009; Inglott et al. 2011; Resch et al. 2011, 2013) and evoked prominent thermoregulatory responses, suggesting a central mediation of the effect. However, taken into account the organization of the thermoregulatory system, which consists of independently operating thermoeffector loops (Romanovsky 2007a, b), the interpretation of the results obtained with one single injection of a substance into the CNS can be misleading, because it can not be excluded that activation of an upstream or downstream peripheral structure of the same thermoregulatory pathway could cause the same or similar effect. For example, Inglott et al. (2011) found that intrathecally administered PACAP 38 exerts sympathoexcitation even after spinal transection and the authors concluded that the effect is, therefore, evoked from the spinal cord itself. Based on the functional architecture of the thermoregulatory system, however, the results by Inglott et al. (2011) can also be explained by a direct effect of PACAP38 on efferent spinal structures (most likely the intermediolateral column) of the same thermoregulatory loop, which can be also activated upstream in the brain by i.c.v. (current study; Pataki et al. 2000, 2003) or by intrahypothalamic infusions (Resch et al. 2011, 2013) and the activation of which leads to equivalent effects regardless of the order of the neuron being activated in the loop. Supporting a peripheral site of action, PACAP38 and its receptor have been shown to be widely expressed on sensory neurons in the periphery (for review, see Mulder et al. 1999; Vaudry et al. 2000) and in the enteric nervous system (Miampamba et al. 2002). In the present study, we compared the effects of central and systemic administration, and showed that the same or even a tenfold lower dose of PACAP38 evokes stronger hyperthermia, when given i.c.v. than when delivered i.v. This result unequivocally supports the central mediation hypothesis. The slight increase of $T_{\mathrm{c}}$ in response to peripherally infused PACAP38 could be attributed to its penetration of the blood-brain barrier (Banks et al. 1993; Nonaka et al. 2002). To our knowledge, this is the first report in which the central and peripheral thermoregulatory responses to PACAP38 were compared under identical experimental conditions.

We also studied the characteristics of the thermoregulatory response to PACAP38 and found that the hyperthermia started to develop promptly: already $10 \mathrm{~min}$ after drug administration, we could detect the activation of thermoeffectors and a slight increase of $T_{\mathrm{c}}$. This is a novel finding of the study as in all of the earlier studies investigating the thermal effect of PACAP38, $T_{\mathrm{b}}$ was recorded hourly and the substance was administered in a stressful manner, thus the developing stress-induced hyperthermia, which was also present in the vehicle-treated animals masked the early phase of the response. We found that PACAP38 administration resulted in the simultaneous activation of non-shivering thermogenesis and cutaneous vasoconstriction, which are the two principal autonomic cold-defense thermoeffectors (Romanovsky 2007a). It has to be noted that the measured increase in $\mathrm{VO}_{2}$ can theoretically originate from elevation of both shivering and non-shivering thermogenesis; but in small rodents, nonshivering thermogenesis is the primary source of heat production (for review, see Cannon and Nedergaard 2004). The initial skin vasoconstriction seems to contradict the reported vasodilatory effect of PACAP38 (Absood et al. 1992; Tsueshita et al. 2002), but this contradiction can be resolved by considering that in the current study PACAP38 was delivered into the lateral ventricle of the brain, from where it can broadly access the POA, where neurons of the thermoeffector pathway for tail skin vasomotor tone are situated (Nakamura and Morrison 2008a, b). Therefore, it is plausible that PACAP3 8 acted on central thermoregulatory elements resulting in skin vasoconstriction, but when the peptide spread to more distant (non-thermoregulatory) areas, it caused vasodilatory effect, which was also observed in the current study 40 min after PACAP38 injection (Fig. 1). Although the later occurring cutaneous vasodilation lasted longer than the initial skin vasonconstriction, from a thermoregulatory point of view, the initial decrease of heat loss is equally important, as it was present during the developmental phase of PACAP38-induced hyperthermia, thus contributed to the rise of deep $T_{\mathrm{b}}$.

Since neurons of the thermoeffector loop for non-shivering thermogenesis are also located in the POA (for review, see Romanovsky et al. 2009), it is tempting to assume that the site of the hyperthermic effect of PACAP38 is in the POA on neurons, which belong to the common part of the thermoeffector pathways for non-shivering thermogenesis and cutaneous vasoconstriction. Supporting this hypothesis, the PAC1 receptor, which has been shown to be involved in mediation of the hyperthermic effect of PACAP38 (Tachibana et al. 2007; Resch et al. 2013), is abundantly expressed in the MnPO of the POA (Joo et al. 2004), where GABAergic neurons controlling autonomic cold-defense thermoeffectors can be found (Nakamura and Morrison 2008a). In recent studies from Resch et al. (2011, 2013), it has been proposed that the hyperthermic and hypermetabolic effects of PACAP38 are mediated by neurons in the hypothalamic ventromedial nucleus and possibly in the lateral parabrachial nucleus. Our hypothesis is also in harmony with these results, because in the cold-activated pathway glutamatergic neurons from the lateral parabrachial nucleus project to GABAergic neurons in the MnPO (Nakamura and Morrison 2008b), which in turn, are connected to neurons of the hypothalamic 
ventromedial nucleus (Imai-Matsumura et al. 1988; Thornhill et al. 1994).

As an alternative approach to study the role of PACAP in thermoregulation, we investigated deep $T_{\mathrm{b}}$ and locomotor activity of Pacap $^{-/-}$mice and found that these mice were hyperactive throughout the day and hyperthermic during the light phase as compared to controls. The hyperactivity of Pacap $^{-1-}$ mice was also observed in an earlier study (Hashimoto et al. 2001) and, although Adams et al. (2008) reported no alteration in the locomotor activity of the Pacap $^{-1-}$ mice compared to controls, in their study during the three consecutive nights of the experiments the number of beam breaks in case of the $\mathrm{Pacap}^{-/}$mice exceeded by $\sim 2,000$ (i.e., by $\sim 50 \%$ ) that of controls at certain time points. It is a novel finding of the present study that in our experiments, the increased activity of the Pacap $^{-/-}$mice resulted in elevated $T_{\mathrm{b}}$ during the light phase of the day, in which phase locomotor activity correlates strongly with $T_{\mathrm{b}}$ (Weinert and Waterhouse 1998, 1999). In contrast to our findings, in the study by Hashimoto et al. (2009) Pacap $^{-/-}$mice had lower $T_{\mathrm{b}}$ during the night than controls, but those experiments were conducted at a $T_{\mathrm{a}}$ of $23{ }^{\circ} \mathrm{C}$, which could be presumably below the thermoneutral zone of mice. As it has been repeatedly shown that cold-defense responses of Pacap $^{-1}$ mice are impaired compared with controls (Gray et al. 2002; Adams et al. 2008; Cummings et al. 2008), it can be assumed that the different influence of a chronic, mild cold exposure on the $T_{\mathrm{b}}$ of $\mathrm{Pacap}^{-/-}$and $\mathrm{Pacap}^{+/+}$mice could contribute to the observed lower $T_{\mathrm{b}}$ in the Pacap ${ }^{-/-}$mice. Indeed, inadequate heat production and lower $T_{\mathrm{b}}$ in Pacap $^{-/-}$mice were observed in response to chronic, mild $\left(21^{\circ} \mathrm{C}\right)$ cold exposure in the study by Gray et al. (2002). In an earlier study by Cummings et al. (2008), the $T_{\mathrm{b}}$ of Pacap $^{-/-}$mice did not significantly differ from that of controls, however, in that study the authors measured rectal temperature in previously decapitated mice, which method is not sensitive enough to detect small (especially locomotion-induced) differences in $T_{\mathrm{b}}$. Locomotor activity is widely viewed as a thermoregulatory effector in mice (Kanizsai et al. 2009; Szentirmai et al. 2010; Garami et al. 2011) and our findings suggest that freely moving Pacap $^{-/-}$mice utilize locomotor activity as a thermoeffector to maintain an elevated $T_{\mathrm{b}}$ during the light phase and normal $T_{\mathrm{b}}$ during the night phase of the day. It has to be mentioned that the increased locomotor activity of the $\mathrm{Pacap}^{-/-}$mice could possibly also originate from distinct mechanisms, which are independent from thermoregulation.

We then asked whether the basal daytime $T_{\mathrm{b}}$ of loosely restrained $\mathrm{PaCap}^{-/-}$mice (i.e., those that can not use locomotion as a thermoeffector) also differs from their wildtype littermates. In contrast to our results in freely moving mice, when restrained, Pacap $^{-/}$mice were hypometabolic and had lower $T_{\mathrm{b}}$ than controls. The decreased metabolic rate and $T_{\mathrm{b}}$ in the absence of PACAP is in harmony with our results demonstrating the hypermetabolic and hyperthermic effect of PACAP38 injection in rats. When we measured the expression of c-Fos positive cells in the POA of the mice, we found that the number of c-Fos positive cells in the MPO was markedly higher in Pacap $^{-1}$ mice than in controls, suggesting that the absence of PACAP results in an increased activation of MPO neurons. Since GABAergic neurons in the MPO tonically suppress thermogenesis (Osaka 2004), we propose that in Pacap $^{-/}$ mice inhibitory MPO neurons are more activated and this results in an enhanced suppression of thermogenesis. This hypothesis is also in harmony with the proposed action of PACAP38 injection on GABAergic MnPO neurons (see above), because activation of these neurons results in an increased inhibition of the inhibitory MPO neurons, which leads to elevated metabolic rate and hyperthermia. Although alternate explanations are also plausible, it can be assumed that the absence of PACAP38 results in a lower resting metabolic rate (and $T_{\mathrm{b}}$ ) and as a compensatory mechanism for the hypometabolism, Pacap $^{-/-}$mice become hyperkinetic to maintain normal (or even higher) $T_{\mathrm{b}}$. Interestingly, a similarly altered thermoeffector pattern (hypometabolism and hyperkinesis) was observed in our recent study with mice lacking the TRPV1 channel (Garami et al. 2011). The similar thermoregulatory consequences of the absence of PACAP and TRPV1 can be explained with the alteration of the same neural pathways as PACAP38 is released from activated capsaicin-sensitive (i.e., TRPV1-expressing) neural afferents into the systemic circulation (Helyes et al. 2007). Although the exact molecular and neuronal mechanisms involved in the development of the observed thermoregulatory phenotype of Pacap $^{-/}$ mice need to be further investigated, an involvement of altered biochemical processes in the CNS of Pacap $^{-/-}$mice can be suspected (Maasz et al. 2014).

In conclusion, we showed in a straightforward comparative experiment that PACAP38 causes hyperthermia by acting on targets within the CNS. The PACAP38-induced hyperthermia is brought about through the simultaneous activation of both autonomic cold-defense effectors: elevation of non-shivering thermogenesis and cutaneous vasoconstriction. We hypothesize that GABAergic neurons within the MnPO are involved in mediation of thermoregulatory response to PACAP38. The absence of PACAP results in hyperkinesis and daytime hyperthermia in freely-moving Pacap $^{-1}$ mice through mechanisms which need to be clarified, but an involvement of TRPV1 and altered central biochemical processes can be suspected. The increased locomotor activity is presumably a compensatory mechanism for the hypometabolism and hypothermia, which is present under resting conditions in the absence of PACAP. 
Acknowledgments This research has been supported by the Hungarian Scientific Research Fund (grants PD 105532, PD 100706, and K 104984), the Janos Bolyai Research Scholarship of the Hungarian Academy of Sciences (BO/00785/12/5), the TAMOP (grants 4.2.2.A-11/1/ KONV-2012-0024 and 4.2.4.A/2-11-1-2012-0001), the Arimura Foundation, the PTE-MTA "Lendulet" Program, and the Hungarian Brain Research Program (grant KTIA_13_NAP-A-III/5).

\section{References}

Absood A, Chen D, Wang ZY, Hakanson R (1992) Vascular effects of pituitary adenylate cyclase activating peptide: a comparison with vasoactive intestinal peptide. Regul Pept 40:323-329

Adams BA, Gray SL, Isaac ER, Bianco AC, Vidal-Puig AJ, Sherwood NM (2008) Feeding and metabolism in mice lacking pituitary adenylate cyclase-activating polypeptide. Endocrinology 149:15711580

Almeida MC, Hew-Butler T, Soriano RN et al (2012) Pharmacological blockade of the cold receptor TRPM8 attenuates autonomic and behavioral cold defenses and decreases deep body temperature. J Neurosci 32:2086-2099

Aschoff J, von Saint-Paul U (1973) Brain temperature as related to gross motor activity in the unanesthetized chicken. Physiol Behav 10: $529-533$

Balasko M, Garami A, Soos S, Koncsecsko-Gaspar M, Szekely M, Petervari E (2010) Central alpha-MSH, energy balance, thermal balance, and antipyresis. J Therm Biol 35:211-217

Banki E, Degrell P, Kiss P et al (2013) Effect of PACAP treatment on kidney morphology and cytokine expression in rat diabetic nephropathy. Peptides 42:125-130

Banki E, Kovacs K, Nagy D et al (2014) Molecular mechanisms underlying the nephroprotective effects of PACAP in diabetes. $\mathrm{J}$ Mol Neurosci (in press)

Banks WA, Kastin AJ, Komaki G, Arimura A (1993) Passage of pituitary adenylate cyclase activating polypeptide $1-27$ and pituitary adenylate cyclase activating polypeptide1-38 across the blood-brain barrier. J Pharmacol Exp Ther 267:690-696

Brown D, Livesey G, Dauncey MJ (1991) Influence of mild cold on the components of 24 hour thermogenesis in rats. J Physiol 441: $137-154$

Brown D, Tamas A, Reglodi D, Tizabi Y (2013) PACAP protects against salsolinol-induced toxicity in dopaminergic SH-SY5Y cells: implication for Parkinson's disease. J Mol Neurosci 50:600-607

Brown D, Tamas A, Reglodi D, Tizabi Y. (2014) PACAP protects against inflammatory-mediated toxicity in dopaminergic SHSY5Y cells: implication for Parkinson's disease. Neurotox Res (in press)

Cannon B, Nedergaard J (2004) Brown adipose tissue: function and physiological significance. Physiol Rev 84:277-359

Cummings KJ, Willie C, Wilson RJ (2008) Pituitary adenylate cyclaseactivating polypeptide maintains neonatal breathing but not metabolism during mild reductions in ambient temperature. Am J Physiol Regul Integr Comp Physiol 294:R956-R965

Danyadi B, Szabadfi K, Reglodi D et al (2014) PACAP application improves functional outcome of chronic retinal ischemic injury in rats-evidence from electroretinographic measurements. J Mol Neurosci (in press)

Das M, Vihlen CS, Legradi G (2007) Hypothalamic and brainstem sources of pituitary adenylate cyclase-activating polypeptide nerve fibers innervating the hypothalamic paraventricular nucleus in the rat. J Comp Neurol 500:761-776 de Oliveira C, Garami A, Lehto SG et al (2014) Transient receptor potential channel ankyrin-1 is not a cold sensor for autonomic thermoregulation in rodents. J Neurosci 34:4445-4452

Garami A, Shimansky YP, Pakai E, Oliveira DL, Gavva NR, Romanovsky AA (2010) Contributions of different modes of TRPV1 activation to TRPV1 antagonist-induced hyperthermia. J Neurosci 30:1435-1440

Garami A, Pakai E, Oliveira DL et al (2011) Thermoregulatory phenotype of the Trpv1 knockout mouse: thermoeffector dysbalance with hyperkinesis. J Neurosci 31:1721-1733

Gasz B, Racz B, Roth E et al (2006) Pituitary adenylate cyclase activating polypeptide protects cardiomyocytes against oxidative stressinduced apoptosis. Peptides 27:87-94

Gaszner B, Kormos V, Kozicz T, Hashimoto H, Reglodi D, Helyes Z (2012) The behavioral phenotype of pituitary adenylate-cyclase activating polypeptide-deficient mice in anxiety and depression tests is accompanied by blunted c-Fos expression in the bed nucleus of the stria terminalis, central projecting Edinger-Westphal nucleus, ventral lateral septum, and dorsal raphe nucleus. Neuroscience 202:283-299

Gray SL, Yamaguchi N, Vencova P, Sherwood NM (2002) Temperaturesensitive phenotype in mice lacking pituitary adenylate cyclaseactivating polypeptide. Endocrinology 143:3946-3954

Hashimoto H, Shintani N, Tanaka K et al (2001) Altered psychomotor behaviors in mice lacking pituitary adenylate cyclase-activating polypeptide (PACAP). Proc Natl Acad Sci U S A 98:13355-13360

Hashimoto H, Hashimoto R, Shintani N et al (2009) Depression-like behavior in the forced swimming test in PACAP-deficient mice: amelioration by the atypical antipsychotic risperidone. J Neurochem 110:595-602

Hawke Z, Ivanov TR, Bechtold DA, Dhillon H, Lowell BB, Luckman SM (2009) PACAP neurons in the hypothalamic ventromedial nucleus are targets of central leptin signaling. J Neurosci 29: $14828-14835$

Helyes Z, Pozsgai G, Borzsei R et al (2007) Inhibitory effect of PACAP38 on acute neurogenic and non-neurogenic inflammatory processes in the rat. Peptides 28:1847-1855

Imai-Matsumura K, Matsumura K, Tsai CL, Nakayama T (1988) Thermal responses of ventromedial hypothalamic neurons in vivo and in vitro. Brain Res 445:193-197

Inglott MA, Farnham MM, Pilowsky PM (2011) Intrathecal PACAP-38 causes prolonged widespread sympathoexcitation via a spinally mediated mechanism and increases in basal metabolic rate in anesthetized rat. Am J Physiol Heart Circ Physiol 300:H2300-H2307

Joo KM, Chung YH, Kim MK et al (2004) Distribution of vasoactive intestinal peptide and pituitary adenylate cyclase-activating polypeptide receptors (VPAC1, VPAC2, and PAC1 receptor) in the rat brain. J Comp Neurol 476:388-413

Kanizsai P, Garami A, Solymar M, Szolcsanyi J, Szelenyi Z (2009) Energetics of fasting heterothermia in TRPV1-KO and wild type mice. Physiol Behav 96:149-154

Kemeny A, Reglodi D, Cseharovszky R et al (2010) Pituitary adenylate cyclase-activating polypeptide deficiency enhances oxazoloneinduced allergic contact dermatitis in mice. J Mol Neurosci 42: 443-449

Koves K, Kantor O, Lakatos A, et al (2014) Advent and recent advances in research on the role of pituitary adenylate cyclase-activating polypeptide (PACAP) in the regulation of gonadotropic hormone secretion of female rats. J Mol Neurosci (in press)

Lenti L, Domoki F, Kis D et al (2007) Pituitary adenylate cyclaseactivating polypeptide induces pial arteriolar vasodilation through cyclooxygenase-dependent and independent mechanisms in newborn pigs. Brain Res 1165:81-88

Maasz G, Pirger Z, Reglodi D et al (2014) Comparative protein composition of the brains of PACAP-deficient mice using mass spectrometry-based proteomic analysis. J Mol Neurosci (in press) 
Miampamba M, Germano PM, Arli S et al (2002) Expression of pituitary adenylate cyclase-activating polypeptide and PACAP type 1 receptor in the rat gastric and colonic myenteric neurons. Regul Pept 105: $145-154$

Miyata A, Arimura A, Dahl RR et al (1989) Isolation of a novel 38 residue-hypothalamic polypeptide which stimulates adenylate cyclase in pituitary cells. Biochem Biophys Res Commun 164:567-574

Miyata A, Jiang L, Dahl RD et al (1990) Isolation of a neuropeptide corresponding to the N-terminal 27 residues of the pituitary adenylate cyclase activating polypeptide with 38 residues (PACAP38). Biochem Biophys Res Commun 170:643-648

Mount LE, Willmott JV (1967) The relation between spontaneous activity, metabolic rate and the 24 hour cycle in mice at different environmental temperatures. J Physiol 190:371-380

Mulder H, Jongsma H, Zhang Y, Gebre-Medhin S, Sundler F, Danielsen N (1999) Pituitary adenylate cyclase-activating polypeptide and islet amyloid polypeptide in primary sensory neurons: functional implications from plasticity in expression on nerve injury and inflammation. Mol Neurobiol 19:229-253

Nagy AD, Csernus VJ (2007) The role of PACAP in the control of circadian expression of clock genes in the chicken pineal gland. Peptides 28:1767-1774

Nakamura K, Morrison SF (2008a) Preoptic mechanism for colddefensive responses to skin cooling. J Physiol 586:2611-2620

Nakamura K, Morrison SF (2008b) A thermosensory pathway that controls body temperature. Nat Neurosci 11:62-71

Njaine B, Rocha-Martins M, Vieira-Vieira CH et al (2014) Pleiotropic functions of pituitary adenylyl cyclase-activating polypeptide on retinal ontogenesis: involvement of KLF4 in the control of progenitor cell proliferation. J Mol Neurosci (in press)

Nonaka N, Banks WA, Mizushima H, Shioda S, Morley JE (2002) Regional differences in PACAP transport across the blood-brain barrier in mice: a possible influence of strain, amyloid beta protein, and age. Peptides 23:2197-2202

Nowak JZ, Kuba K (2002) Pituitary adenylate cyclase-activating polypeptide and vasoactive intestinal peptide-stimulated cyclic AMP synthesis in rat cerebral cortical slices: interaction with noradrenaline, adrenaline, and forskolin. J Mol Neurosci 18:4752

Osaka T (2004) Cold-induced thermogenesis mediated by GABA in the preoptic area of anesthetized rats. Am J Physiol Regul Integr Comp Physiol 287:R306-R313

Palkovits M, Somogyvari-Vigh A, Arimura A (1995) Concentrations of pituitary adenylate cyclase activating polypeptide (PACAP) in human brain nuclei. Brain Res 699:116-120

Pataki I, Adamik A, Jaszberenyi M, Macsai M, Telegdy G (2000) Pituitary adenylate cyclase-activating polypeptide induces hyperthermia in the rat. Neuropharmacology 39:1303-1308

Pataki I, Adamik A, Jaszberenyi M, Macsai M, Telegdy G (2003) Involvement of transmitters in pituitary adenylate cyclaseactivating polypeptide-induced hyperthermia. Regul Pept 115: 187-193

Paxinos G, Franklin KBJ (2004) The mouse brain in stereotaxic coordinates, Second Edition (ed). Academic Press, San Diego, CA

Petervari E, Garami A, Pakai E, Szekely M (2005) Effects of perineural capsaicin treatment of the abdominal vagus on endotoxin fever and on a non-febrile thermoregulatory event. J Endotoxin Res 11:260266

Petervari E, Balasko M, Garami A, Soos S, Szekely M (2009) Suppression of food intake by intracerebroventricular injection of alpha-MSH varies with age in rats. Acta Physiol Hung 96: 483-487

Petervari E, Garami A, Soos S, Szekely M, Balasko M (2010) Agedependence of alpha-MSH-induced anorexia. Neuropeptides 44: 315-322
Racz B, Horvath G, Faluhelyi N et al (2008) Effects of PACAP on the circadian changes of signaling pathways in chicken pinealocytes. J Mol Neurosci 36:220-226

Reglodi D, Somogyvari-Vigh A, Vigh S, Kozicz T, Arimura A (2000) Delayed systemic administration of PACAP38 is neuroprotective in transient middle cerebral artery occlusion in the rat. Stroke 31:1411-1417

Resch JM, Boisvert JP, Hourigan AE, Mueller CR, Yi SS, Choi S (2011) Stimulation of the hypothalamic ventromedial nuclei by pituitary adenylate cyclase-activating polypeptide induces hypophagia and thermogenesis. Am J Physiol Regul Integr Comp Physiol 301: R1625-R1634

Resch JM, Maunze B, Gerhardt AK, Magnuson SK, Phillips KA, Choi S (2013) Intrahypothalamic pituitary adenylate cyclase-activating polypeptide regulates energy balance via site-specific actions on feeding and metabolism. Am J Physiol Endocrinol Metab 305: E1452-E1463

Romanovsky AA (2007a) Temperature regulation. Chapter 23. In: Petersen $\mathrm{O}$ (ed) Lecture notes on human physiology, 5th edn. Blackwell, Oxford, pp 603-615

Romanovsky AA (2007b) Thermoregulation: some concepts have changed. Functional architecture of the thermoregulatory system. Am J Physiol Regul Integr Comp Physiol 292:R37-R46

Romanovsky AA (2014) Skin temperature: its role in thermoregulation. Acta Physiol 210:498-507

Romanovsky AA, Ivanov AI, Shimansky YP (2002) Selected contribution: ambient temperature for experiments in rats: a new method for determining the zone of thermal neutrality. J Appl Physiol 92:2667-2679

Romanovsky AA, Almeida MC, Garami A et al (2009) The transient receptor potential vanilloid-1 channel in thermoregulation: a thermosensor it is not. Pharmacol Rev 61:228-261

Rozzi S.J, Borelli G, Ryan K, et al (2014) PACAP27 is protective against Tat-induced neurotoxicity. J Mol Neurosci (in press)

Ryabinin AE, Wang YM, Finn DA (1999) Different levels of Fos immunoreactivity after repeated handling and injection stress in two inbred strains of mice. Pharmacol Biochem Behav 63:143-151

Sagar SM, Sharp FR, Curran T (1988) Expression of c-fos protein in brain: metabolic mapping at the cellular level. Science 240: $1328-1331$

Seeliger S, Buddenkotte J, Schmidt-Choudhury A et al (2010) Pituitary adenylate cyclase activating polypeptide: an important vascular regulator in human skin in vivo. Am J Pathol 177:2563-2575

Szentirmai E, Kapas L, Sun Y, Smith RG, Krueger JM (2010) Restricted feeding-induced sleep, activity, and body temperature changes in normal and preproghrelin-deficient mice. Am J Physiol Regul Integr Comp Physiol 298:R467-R477

Tachibana T, Oikawa D, Adachi N, Boswell T, Furuse M (2007) Central administration of vasoactive intestinal peptide and pituitary adenylate cyclase-activating polypeptide differentially regulates energy metabolism in chicks. Comp Biochem Physiol A Mol Integr Physiol 147:156-164

Thornhill J, Jugnauth A, Halvorson I (1994) Brown adipose tissue thermogenesis evoked by medial preoptic stimulation is mediated via the ventromedial hypothalamic nucleus. Can J Physiol Pharmacol 72:1042-1048

Tsueshita T, Gandhi S, Onyuksel H, Rubinstein I (2002) Phospholipids modulate the biophysical properties and vasoactivity of PACAP-(138). J Appl Physiol 93:1377-1383

Vaudry D, Gonzalez BJ, Basille M, Fournier A, Vaudry H (1999) Neurotrophic activity of pituitary adenylate cyclase-activating polypeptide on rat cerebellar cortex during development. Proc Natl Acad Sci U S A 96:9415-9420

Vaudry D, Gonzalez BJ, Basille M, Yon L, Fournier A, Vaudry H (2000) Pituitary adenylate cyclase-activating polypeptide and its receptors: from structure to functions. Pharmacol Rev 52:269-324 
Vaudry D, Falluel-Morel A, Bourgault S et al (2009) Pituitary adenylate cyclase-activating polypeptide and its receptors: 20 years after the discovery. Pharmacol Rev 61:283-357

Weinert D, Waterhouse J (1998) Diurnally changing effects of locomotor activity on body temperature in laboratory mice. Physiol Behav 63: 837-843
Weinert D, Waterhouse J (1999) Daily activity and body temperature rhythms do not change simultaneously with age in laboratory mice. Physiol Behav 66:605-612

Wilhelm I, Fazakas C, Tamas A, Toth G, Reglodi D, Krizbai IA (2014) PACAP enhances barrier properties of cerebral microvessels. J Mol Neurosci (in press) 
IV. 


\title{
Aging reverses the role of the transient receptor potential vanilloid-1 channel in systemic inflammation from anti-inflammatory to proinflammatory
}

\author{
Samuel P. Wanner, ${ }^{1,2}$ Andras Garami,, Eszter Pakai,' Daniela L. Oliveira, ${ }^{1}$ Narender R. Gavva, ${ }^{3}$ Cândido C. Coimbra ${ }^{2}$ \\ and Andrej A. Romanovsky',*
}

'Systemic Inflammation Laboratory (FeverLab); Trauma Research; St. Joseph's Hospital and Medical Center; Phoenix, AZ USA; ${ }^{2}$ Department of Physiology and Biophysics; Federal University of Minas Gerais; Minas Gerais, Brazil; ${ }^{3}$ Department of Neuroscience; Amgen; Thousand Oaks, CA USA

Key words: TRP channels, sepsis, systemic inflammation, endotoxin shock

Abbreviations: CLP, cecal ligation and puncture; LPS, lipopolysaccharide; SIRS, systemic inflammatory response syndrome; $T_{b}$, body temperature; TNF, tumor necrosis factor; TRPV1, transient receptor potential vanilloid-1

\begin{abstract}
Studies in young rodents have shown that the transient receptor potential vanilloid-1 (TRPV1) channel plays a suppressive role in the systemic inflammatory response syndrome (SIRS) by inhibiting production of tumor necrosis factor (TNF) $\alpha$ and possibly by other mechanisms. We asked whether the anti-inflammatory role of TRPV1 changes with age. First, we studied the effect of AMG517, a selective and potent TRPV1 antagonist, on aseptic, lipopolysaccharide (LPS)-induced SIRS in young (12 wk) mice. In agreement with previous studies, AMG517 increased LPS-induced mortality in the young. We then studied the effects of TRPV1 antagonism (AMG517 or genetic deletion of TRPV1) on SIRS in middle-aged (43$44 \mathrm{wk}$ ) mice. Both types of TRPV1 antagonism delayed and decreased LPS-induced mortality, indicating a reversal of the anti-inflammatory role of TRPV1 with aging. In addition, deletion of TRPV1 decreased the serum TNF $\alpha$ response to LPS, suggesting that the suppressive control of TRPV1 on TNF $\alpha$ production is also reversed with aging. In contrast to aseptic SIRS, polymicrobial sepsis (induced by cecal ligation and puncture) caused accelerated mortality in aged TRPV1-deficient mice as compared with wild-type littermates. The recovery of TRPV1-deficient mice from hypothermia associated with the cecal ligation and puncture procedure was delayed. Hence, the reversal of the anti-inflammatory role of TRPV1 found in the aged and their decreased systemic inflammatory response are coupled with suppressed defense against microbial infection. These results caution that TRPV1 antagonists, widely viewed as new-generation painkillers, may decrease the resistance of older patients to infection and sepsis.
\end{abstract}

\section{Introduction}

Systemic inflammatory response syndrome (SIRS) is the leading cause of death in hospitalized patients. ${ }^{1,2}$ SIRS is considered a disease of the aged: its incidence and mortality are substantially higher in the older population. ${ }^{3}$ SIRS can be either triggered by non-infectious insults, such as blunt trauma, or associated with an infection (in which case it is called sepsis). In the laboratory, systemic administration of lipopolysaccharide (LPS, a cell-wall constituent of Gram-negative bacteria) in mice and rats is often used to induce SIRS aseptically, whereas polymicrobial sepsis is often studied in rodents subjected to cecal ligation and puncture (CLP). In either model, shock and death can occur, largely as the result of the "cytokine storm," an overt production of proinflammatory cytokines, including TNF $\alpha,{ }^{4}$ and other mediators, cumulatively referred to as the "inflammatory soup." ${ }^{5-7}$ In both
LPS-induced SIRS and CLP-induced sepsis, proinflammatory cytokine production and mortality rate are much lower in young animals. ${ }^{8-11}$ Furthermore, sepsis in young animals is much more responsive to treatment. ${ }^{11}$

Recent studies have brought attention to the role that the transient receptor potential vanilloid-1 (TRPV1) channel may play in SIRS. Abundant on small-diameter sensory nerve fibers, TRPV1 is activated by diverse stimuli, including several ingredients of the inflammatory soup. ${ }^{12,13}$ Activation of TRPV1 on sensory nerves potently inhibits LPS-induced TNF $\alpha$ production. ${ }^{14}$ Studies using either knockout $\left(\operatorname{Trp}_{\mathrm{r}} \mathrm{I}^{-{ }_{-}}\right)$mice, a pharmacological blockade with capsazepine (TRPV1 antagonist) or desensitization with resiniferatoxin (TRPV1 agonist) have shown that TRPV1 plays an anti-inflammatory role in LPS-induced SIRS by, among other mechanisms, limiting the production of TNF $\alpha$, possibly via sensory nerves. ${ }^{15-17}$ However, all studies cited above were conducted 


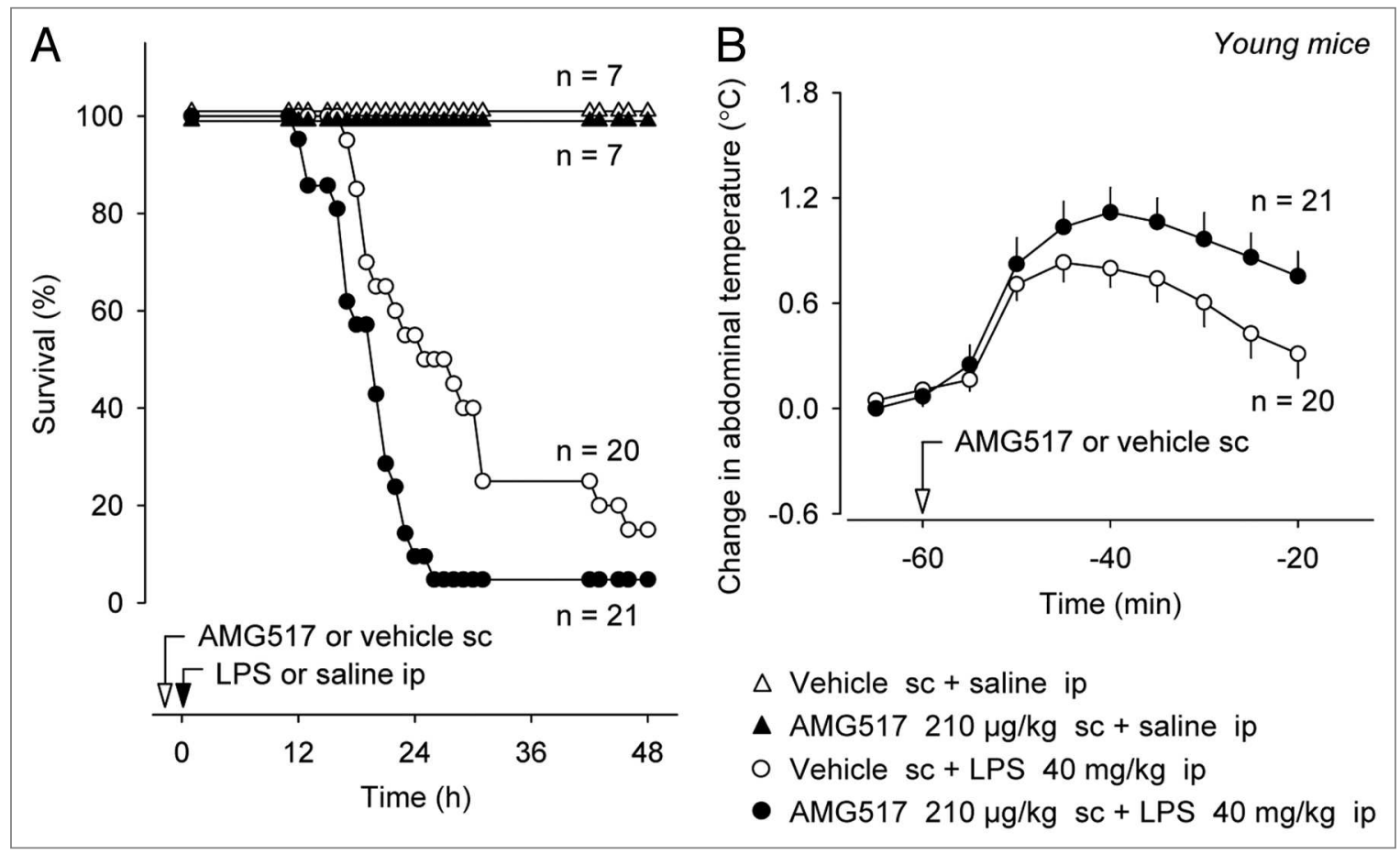

Figure 1. Systemic pretreatment with AMG517 (dose indicated) decreases survival of young mice in LPS-induced SIRS (A). Confirming an effective blockade of TRPV1 channels, the AMG517 pretreatment increases deep $T_{b}$ in young mice (B).

Table 1. Effects of age and TRPV1 antagonism on mortality in LPS-induced SIRS and CLP-induced sepsis

\begin{tabular}{|c|c|c|c|c|c|c|c|c|}
\hline \multirow[t]{2}{*}{ Model } & \multirow[t]{2}{*}{ Variable } & \multicolumn{3}{|c|}{ Cox survival regression analysis } & \multicolumn{2}{|c|}{ Logrank test } & \multicolumn{2}{|c|}{ Time to death } \\
\hline & & $\begin{array}{c}\text { Hazard ratio of } \\
\text { death }\end{array}$ & $\begin{array}{l}\text { 95\% confidence } \\
\text { interval }\end{array}$ & $\mathbf{p}$ & Score & $\mathbf{p}$ & Mean \pm SE $(h)$ & $\mathbf{p}$ \\
\hline LPS & Aged vs. young & 2.2 & $1.3 \div 3.1$ & $<0.001$ & 20.5 & $<0.001$ & $16 \pm 1$ vs. $26 \pm 2$ & $<0.001$ \\
\hline LPS in young & AMG517 vs. vehicle & 0.9 & $0.2 \div 1.6$ & $<0.010$ & 4.1 & $<0.05$ & $19 \pm 1$ vs. $26 \pm 2$ & 0.003 \\
\hline LPS in aged & AMG517 vs. vehicle & -1.0 & $-1.8 \div-0.2$ & $<0.015$ & 5.4 & $<0.05$ & $19 \pm 1$ vs. $16 \pm 1$ & $<0.040$ \\
\hline LPS in aged & $\operatorname{Trpv1} 1^{-/-}$vs. Trpv1 $1^{+/+}$ & -1.3 & $-2.4 \div-0.2$ & $<0.020$ & 5.4 & $<0.05$ & $24 \pm 3$ vs. $19 \pm 1$ & $<0.100$ \\
\hline CLP in aged & $\operatorname{Trpv1^{-/-}}$ vs. $\operatorname{Trpv} 1^{+/+}$ & 0.7 & $-0.5 \div 1.9$ & 0.239 & 1.4 & $>0.05$ & $20 \pm 2$ vs. $52 \pm 11$ & $<0.007$ \\
\hline
\end{tabular}

in young rodents. Whether TRPV1 channels play a similarly prominent anti-inflammatory role in the aged is unknown.

\section{Results and Discussion}

Effects of a TRPV1 antagonist on LPS-induced systemic inflammation in young mice. First, we verified whether pretreatment with AMG517, a potent and selective TRPV1 antagonist, ${ }^{18,19}$ decreases the mortality of young adult (12 wk) C57BL/6 mice in LPS-induced SIRS. Mice responded to LPS $(40 \mathrm{mg} / \mathrm{kg}$, ip) with a marked, rapidly progressing SIRS (Fig. 1A). Pretreatment with AMG517 $(210 \mu \mathrm{g} / \mathrm{kg}$, sc) profoundly decreased the survival rate at multiple time points (e.g., from $50 \%$ to $5 \%$ at $26 \mathrm{~h}$, $\mathrm{p}<0.001$ ), overall $(48 \mathrm{~h})$ survival rate (from $15 \%$ to $5 \%$, p < 0.05 ) and increased the risk of mortality (hazard ratio of death of $0.9, \mathrm{p}=0.01$, Table 1$)$. AMG517 pretreatment also shortened the mean time to death from $26 \pm 2$ to $19 \pm 1 \mathrm{~h}(\mathrm{p}=0.003)$. All
LPS-treated mice, both in this experiment and in other experiments within the present study, developed profound hypothermia: deep (abdominal) body temperature $\left(T_{b}\right)$ decreased from $-36^{\circ} \mathrm{C}$ to $-33^{\circ} \mathrm{C}$ at $10 \mathrm{~h}$. However, no inter-treatment differences in the hypothermic response occurred during the short time period before mice started dying (data not shown). Similar to our previous studies in references 18 and 19, AMG517 caused a short-lasting increase in $T_{b}$ compared with the vehicle $(\mathrm{p}<0.01$, Fig. 1B), thus confirming an effective systemic blockade of TRPV1 channels. Overall, the results of our experiment show that pharmacological blockade of TRPV1 increases mortality of young mice in LPS-induced SIRS. Similar observations have been made in adolescent $(6-8 \mathrm{wk})$ mice and in rats treated with capsazepine. ${ }^{16,17}$ It should be noted, however, that capsazepine is not a highly selective TRPV1 antagonist and has a low potency of blocking the proton mode of TRPV1 activation in the rat and mouse. ${ }^{20}$ In fact, a non-TRPV1-mediated effect of capsazepine 


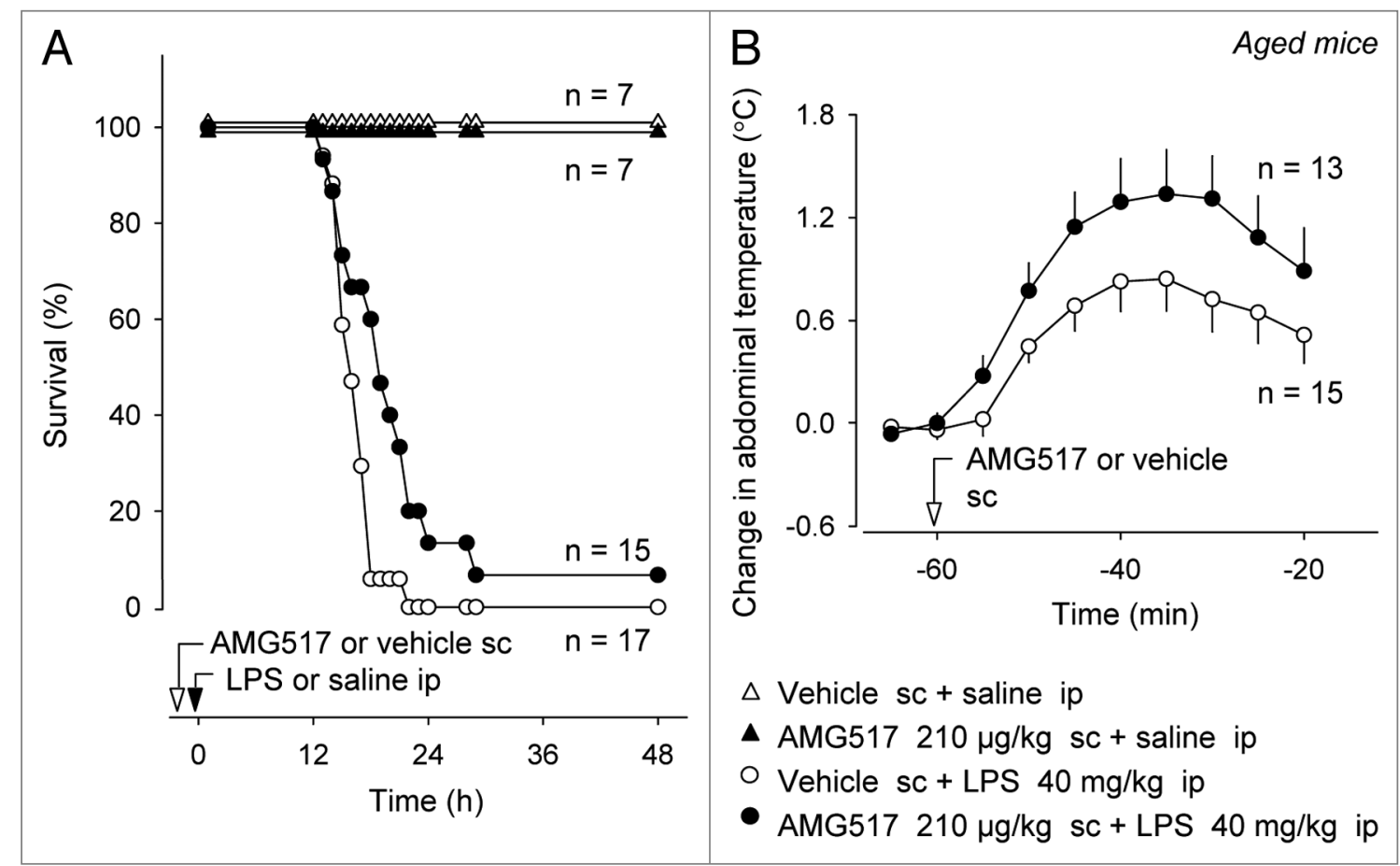

Figure 2. Systemic pretreatment with AMG517 (dose indicated) increases survival of aged mice in LPS-induced SIRS (A). Confirming an effective blockade of TRPV1 channels, the AMG517 pretreatment increases deep $T_{b}$ in aged mice (B).

on the outcome of systemic inflammation has been proposed recently in reference 17 . The present results also agree with the exaggerated symptoms of LPS-induced shock found in young mature (13-20 wk) $\operatorname{Tr}_{p} 1^{-1-}$ mice. ${ }^{15}$

As reviewed by Steiner et al. ${ }^{21}$ and Guptill et al. ${ }^{17}$ many treatments affect mortality in LPS-induced aseptic inflammation and CLP-induced sepsis in opposite ways, confirming that the systemic inflammatory response per se can be harmful to the host, even though it is crucial for defending the host against infection. ${ }^{6,7}$ For example, mice with a dysfunctional toll-like receptor 4 are resistant to LPS but are highly susceptible to Gram-negative bacterial infection. ${ }^{22-24}$ This susceptibility to infection can be reserved by pretreating the toll-like receptor 4-deficient mice with TNF and interleukin- $1 \alpha{ }^{22}$ Interestingly, antibiotic treatment makes sepsis less different from aseptic SIRS, as it eradicates the infectious agent. For example, acute nicotine administration increases survival of mice in LPS-induced SIRS $S^{21,25-27}$ and in antibiotic-treated CLP-induced sepsis, ${ }^{25,26}$ but it worsens the outcome of untreated CLP-induced sepsis in the same species. ${ }^{21}$ From this point of view, two studies of the role of TRPV1 in CLP-induced sepsis in adolescent $(5-8 \mathrm{wk})$ mice $^{17,28}$ agree with the effects observed in LPS SIRS. The first study ${ }^{17}$ has found that antibiotic-treated CLP-induced sepsis causes a higher mortality when TRPV1 channels are absent $\left(\operatorname{Tr} p v 1^{-1-}\right.$ mice) or desensitized (with intrathecal resiniferatoxin). The second study ${ }^{28}$ has found that capsazepine increases survival in untreated CLP-induced sepsis. Overall, prior literature data obtained in young rodents (adolescents to mature adults) and our present experiment with AMG517 in young adult mice show that the effects of TRPV1 blockade on both LPS-induced SIRS and antibiotic-treated sepsis vary from none to strong exaggeration of severity and mortality, ${ }^{15-17}$ whereas the effect of TRPV1 blockade on mortality in untreated sepsis is the opposite: attenuation. ${ }^{28}$

Effects of AMG517 on LPS-induced systemic inflammation in aged mice. To study whether the anti-inflammatory role of TRPV1 in SIRS is preserved with aging, we conducted experiments in middle-aged (44 wk) C57BL/6 mice (Fig. 2A). The outcome of LPS-induced SIRS in these older mice was more severe than in young mice (hazard ratio of $2.2, \mathrm{p}<0.001$, Table 1 ). The mean time to death in vehicle-pretreated aged mice was $16 \pm 1 \mathrm{~h}$, and none of the vehicle-pretreated aged mouse survived for longer than $24 \mathrm{~h}$. Pretreatment of aged mice with AMG517 (210 $\mu \mathrm{g} / \mathrm{kg}, \mathrm{sc})$ increased the survival rate $(\mathrm{p}<0.05)$, delayed the mean time to death $(19 \pm 1 \mathrm{~h}, \mathrm{p}<0.05)$ and decreased the risk of mortality (hazard ratio of $-1.0, \mathrm{p}<0.05$ ) - effects directly opposite of those observed in the young. Survival rate of AMG517-pretreated aged mice at $18 \mathrm{~h}$ was 10 times higher than that of vehicle-pretreated aged mice $(60 \%$ vs. $6 \%, \mathrm{p}<0.001)$. Confirming a systemic blockade of TRPV1 channels in this experiment, AMG517 increased $T_{b}$ as compared with the vehicle ( $\mathrm{p}<0.05$, Fig. 2B). Hence, whereas the effect of AMG517 on LPS-induced systemic inflammation in aged mice was the opposite to that found in young mice (Figs. $1 \mathrm{~A}$ and $2 \mathrm{~A}$ ), the effect on $T_{b}$ was qualitatively the same (Figs. 1B and 2B). It is possible that the role of TRPV1 in different functions changes with age in a different way. In the regulation of locomotor activity ${ }^{29,30}$ and inflammation (present results), the role of TRPV1 reverses with age. In the modulation of $T_{b}$ (for mechanisms, review ref. 31), it does not. In the regulation of body mass, TRPV1 channels are either uninvolved ${ }^{29}$ or counteract obesity $^{32}$ in the young but promote obesity in the aged. ${ }^{29,30}$ 


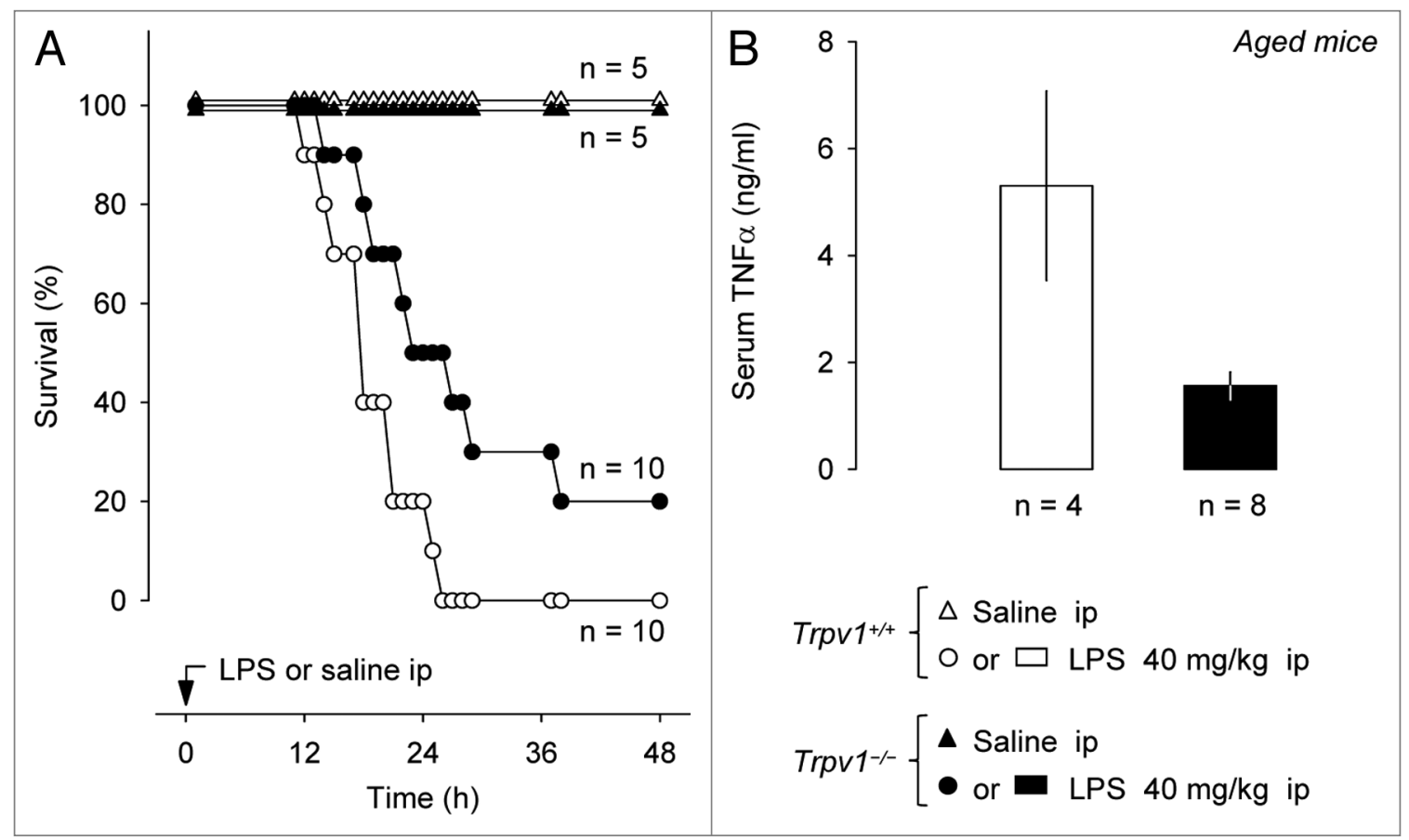

Figure 3. Compared with their age-matched wild-type littermates, middle-aged $\operatorname{Trpv} 7^{-1-}$ mice have a higher survival rate (A) and a lower serum TNF $\alpha$ concentration (B) during LPS-induced SIRS.

Effects of genetic deletion of TRPV1 channels on LPSinduced systemic inflammation in aged mice. We then tested whether genetic deletion of TRPV1 would have the same effects on SIRS in middle-aged mice as a pharmacological blockade. Experiments were conducted in 43-44 wk-old Trpv1 $1^{-1-}$ C57BL/6 129 mice of both sexes and in their age- and sex-matched $\operatorname{Tr} p v 1^{+/+}$littermates. LPS caused death in all $\operatorname{Tr} p v 1^{+l_{+}}$ mice but only in $80 \%$ of $\operatorname{Trpv}^{-1-}$ mice $(\mathrm{p}<0.05$, Fig. 3A). Survival rate of $\operatorname{Trpv1^{-1}}$ mice at $21 \mathrm{~h}$ was 3.5 times higher than of wild-type controls $(70 \%$ vs. $20 \%$, p < 0.001). Genetic deletion of TRPV1 channels decreased the risk of mortality (hazard ratio of $-1.3, \mathrm{p}<0.05)$ and tended to delay death ( $<<0.1$, Table 1$)$. Importantly, $\operatorname{Tr} p v 1^{-1-}$ mice exhibited a $70 \%$ suppression of the TNF $\alpha$ response at $12 \mathrm{~h}$ post-LPS ( $<<0.05$, Fig. 3B). It should be noted that aged $\operatorname{Trpv1}^{-/-}$mice in this study (see Materials and Methods) and in our previous studies in references 29 and 30 were overweight compared with their wild-type littermates. Obesity $^{33-36}$ and hyperlipidemia ${ }^{37}$ associated with various mutations in rats do not seem to affect the febrile response to low, non-shock-inducing doses of LPS, and obesity does not seem to increase the risk of sepsis even though it increases the risk of an infection. ${ }^{38}$ Nevertheless, systemic inflammation and obesity are intimately interconnected, ${ }^{39,40}$ and we cannot rule out that obesity could have been a contributing factor to at least some of the effects found in aged Trpv1 $1^{-1-}$ mice.

Mechanisms of the age-associated reversal of the anti-inflammatory role of TRPV1 are unknown. Our TNF $\alpha$ data suggest that the reversal occurs at initial stages of the pathogenesis of SIRS - at or upstream of TNF $\alpha$ production. The TNF $\alpha$ response to LPS has been shown to be under suppressive control of TRPV1 channels on sensory nerves. ${ }^{14}$ Loss of TRPV1-mediated suppression of TNF $\alpha$ production in aged animals may reflect reduced translation of the TRPV1 protein and its reduced transport to the periphery, ${ }^{41}$ possibly due to age-associated decline in neurotrophic support to ganglionic neurons. ${ }^{42}$ Changes in TNF $\alpha$ production may be central to aging-related changes in the pathobiology of sepsis: elderly patients respond to infection, including septic shock, with higher TNF $\alpha,{ }^{43,44}$ and inflammatory cytokine production in intensive-care-unit patients with sepsis is affected by $\mathrm{TNF} \alpha$-related genetic polymorphisms. ${ }^{45}$

Effects of genetic deletion of TRPV1 channels on CLPinduced sepsis in aged mice. Next, we tested whether the attenuation of aseptic SIRS (of LPS-induced TNF $\alpha$ response and mortality) observed in middle-aged $\operatorname{Tr} p v 1^{1^{-1}}$ mice would result in attenuation of the body's defense against CLP-induced polymicrobial infection. CLP sepsis caused substantial mortality in aged mice of both genotypes (Fig. 4A). However, $\operatorname{Tr} p v 1^{-1-}$ mice died significantly faster than their $\operatorname{Tr} p v 1^{+/+}$littermates (Table 1). The mean time to death in $\operatorname{Tr} p v 1^{-1-}$ mice was $20 \pm 2 \mathrm{~h}$, as compared with $52 \pm 11 \mathrm{~h}$ in $\operatorname{Trpv1} 1^{+/+}$mice $(\mathrm{p}<0.01)$, and the $30 \mathrm{~h}$ survival rate in $\operatorname{Trp} v 1^{+/+}$mice was 3.9 times higher than in $\operatorname{Tr} p v 1^{T_{-}^{-}}$mice ( $86 \%$ vs. $22 \%, p<0.001$ ). Further confirming the higher severity of sepsis in $\operatorname{Trpv1^{-1}}$ mice, recovery of deep $T_{b}$ after the CLP procedure (and the related anesthesia) was delayed ( $p<0.001$, Fig. 4B).

Conclusions. The present study shows that the anti-inflammatory role firmly established for TRPV1 channels in young rodents ${ }^{15-17}$ is reversed with aging. Whereas pharmacological or genetic TRPV1 antagonism decreases the survival rate in aseptic SIRS and in antibiotic-treated sepsis in the young, both types of TRPV1 antagonism have the opposite effect on aseptic SIRS 


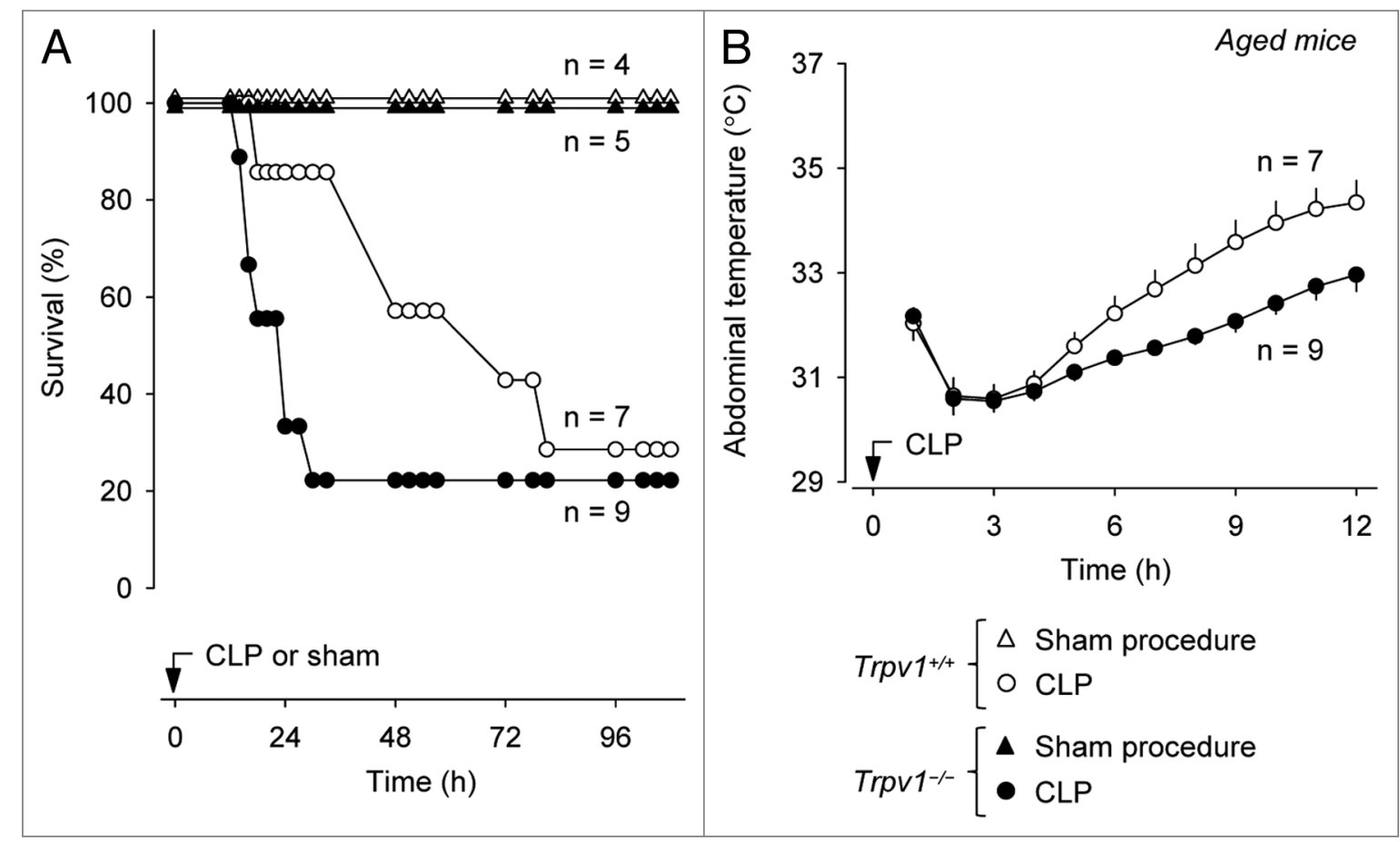

Figure 4. Compared with their age-matched wild-type littermates, middle-aged $\operatorname{Trpv} 7^{--}$mice have a shorter survival time (A) and a slower $T_{b}$ recovery (B) during CLP-induced sepsis.

in middle-aged mice. The age-dependent reversal of the antiinflammatory role of TRPV1 to proinflammatory is likely due, at least in part, to a reversal of the suppressive control of TRPV1 on TNF $\alpha$ production. These pathobiological changes are highly important, as evident from the decreased ability of aged Trpv1 1- mice to resist polymicrobial sepsis. The reversal of the antiinflammatory role of TRPV1 reported in this study is another example of profound effects of aging on the pathobiology of systemic inflammation. ${ }^{11,46,47}$

Significance. Our findings may influence development of TRPV1 antagonists, widely viewed as new-generation painkillers. ${ }^{18,20,48}$ If what we found for murine models applies to human sepsis, anti-TRPV1 therapy may suppress the systemic inflammatory response in the previously uninfected (untreated with antibiotics) elderly and, hence, decrease their resistance to bacterial infection and sepsis. This potential side effect is especially serious, because recognition of infection is often complicated in older patients by a variety of factors, including the absence of fever, which often delays treatment. ${ }^{49,50}$

\section{Materials and Methods}

The study was conducted in 168 adult mice (either young or middle-aged) of both sexes, including $101 \operatorname{Trp} 1^{+/+} \mathrm{C} 57 \mathrm{BL} / 6$ mice (Charles River Laboratories) and $67 \operatorname{Tr} p v 1^{-I_{-}}$or $\operatorname{Tr} p v 1^{+/+} \mathrm{C} 57 \mathrm{BL} / 6$ x 129 mice (Amgen colony at Charles River Laboratories). Several phenotypic properties of Trpv1 $1^{-1}$ mice from the Amgen colony have been characterized in our recent studies in references 29 and 30. At the time of experiments, young adult C57BL/6 mice were 12 wk-old, and their body mass was $25 \pm 0 \mathrm{~g}(\mathrm{n}=55)$; middle-aged C57BL/6 mice were 44 wk-old, and their body mass was $32 \pm 1 \mathrm{~g}(\mathrm{n}=46)$. The ages of C $57 \mathrm{BL} / 6$ mice listed are approximate; the vendor filled orders for a specified age, but did not provide the date of birth for each mouse. Middle-aged Trpv1 $1^{+/+}$C57BL/6 x 129 mice were $43 \pm 1$ wk-old $(\mathrm{n}=30)$, and their body mass was $40 \pm 1 \mathrm{~g}$. Middle-aged Trpv1 ${ }^{-/} \mathrm{C} 57 \mathrm{BL} / 6 \mathrm{x}$ 129 mice were $44 \pm 1$ wk-old $(\mathrm{n}=37)$ and significantly heavier $(46 \pm 1 \mathrm{~g} ; \mathrm{p}<0.001)$ than their wild-type littermates. The ages of C57BL/6 x 129 mice were calculated based on the dates of birth, which were known for all mice. Mice were maintained, surgically prepared and habituated to experimental setups as described in our earlier studies. ${ }^{21,29}$ All surgeries were performed under ketamine-xylazine-acepromazine (81.7, 9.3 and $1.2 \mathrm{mg} / \mathrm{kg}$, ip) anesthesia. Antibiotic protection (enrofloxacin, $1.1 \mathrm{mg} / \mathrm{kg}, \mathrm{sc}$ ) was provided, except for animals subjected to CLP. For deep $T_{b}$ measurements, all mice were implanted intraperitoneally with telemetry transmitters (G2 E-Mitter series, Mini Mitter). For CLP, under the same anesthesia, the cecum was pulled out of the abdominal cavity, filled with the intestinal content (by gently squeezing the content from the ascending colon) and ligated with 3-0 silk just distal to the ileocecal junction. The cecal wall was punctured through at the antimesenteric side with a 26-gauge needle. All protocols were approved by the St. Joseph's Hospital and Medical Center Animal Care and Use Committee.

During all experiments, mice were housed singly in their home cages and placed inside a climatic chamber (model 3940, Forma Scientific) with an ambient temperature maintained at $28.0^{\circ} \mathrm{C}$, i.e., within the thermoneutral zone for this experimental setup. ${ }^{29,51}$ Cages were kept on top of telemetry receivers (model ER-4000, Mini Mitter). In the experiments with LPS-induced 
SIRS, the survival rate and $T_{b}$ were monitored for $48 \mathrm{~h}$. To this end, mice were periodically examined for the presence of spontaneous movements and cardiac and respiratory activities. Because deep $T_{b}$ decreases steeply toward the ambient temperature when an animal dies, $T_{b}$ curves were also examined to identify mortality events. In the experiments with CLP-induced sepsis, the same parameters were monitored for $108 \mathrm{~h}$. At the end of experiments, any survivors were euthanized with sodium pentobarbital (200 mg/kg, ip).

In a separate series of experiments (under the same anesthesia as for surgery), blood $(1 \mathrm{ml})$ was collected by cardiac puncture at $12 \mathrm{~h}$ after LPS administration, and mice were euthanized with sodium pentobarbital. The $12 \mathrm{~h}$ time point for blood collection was chosen because aged mice in this model start dying shortly after this point (Fig. 3A). Serum concentration of TNF $\alpha$ was determined by ELISA according to the manufacturer's instructions (SABiosciences, catalog number MEM-004A).

A suspension of E. coli 0111:B4 LPS (Sigma-Aldrich, L2630, $2.5 \mathrm{mg} / \mathrm{ml}$ ) in saline was prepared in advance and stored at $4^{\circ} \mathrm{C}$. To induce SIRS, LPS ( $40 \mathrm{mg} / \mathrm{kg}$ ip) was injected as a bolus; controls received saline.

AMG517 (gift from Amgen) was used to block TRPV1 receptors pharmacologically. This is a highly potent and selective TRPV1 antagonist that had been tested in human patients. ${ }^{18}$ Aliquots of an ethanolic solution of AMG517 (3 mg/ml) were stored at $-80^{\circ} \mathrm{C}$. This stock was diluted with ethanol and saline ex tempore to achieve a $21 \mu \mathrm{g} / \mathrm{ml}$ final concentration of AMG517 in $3.3 \%$ ethanol. AMG517 $(210 \mu \mathrm{g} / \mathrm{kg} \mathrm{sc})$ or its vehicle was administered as a bolus, $1 \mathrm{~h}$ before the administration of LPS (or saline).

\section{References}

1. Brun-Buisson C. The epidemiology of the systemic inflammatory response. Intensive Care Med 2000; 26:64-74; PMID:10786961; http://dx.doi. org/10.1007/s001340051121.

2. Martin GS, Mannino DM, Eaton S, Moss M. The epidemiology of sepsis in the United States from 1979 through 2000. N Engl J Med 2003; 348:154654; PMID:12700374; http://dx.doi.org/10.1056/ NEJMoa022139.

3. Martin GS, Mannino DM, Moss M. The effect of age on the development and outcome of adult sepsis. Crit Care Med 2006; 34:15-21; PMID:16374151; http:// dx.doi.org/10.1097/01.CCM.0000194535.82812.BA.

4. Töllner B, Roth J, Storr B, Martin D, Voigt K, Zeisberger $\mathrm{E}$. The role of tumor necrosis factor (TNF) in the febrile and metabolic responses of rats to intraperitoneal injection of a high dose of lipopolysaccharide. Pflugers Arch 2000; 440:925-32; PMID:11041560; http://dx.doi.org/10.1007/s004240000386.

5. Cohen J. The immunopathogenesis of sepsis. Nature 2002; 420:885-91; PMID:12490963; http://dx.doi. org/10.1038/nature01326.

6. Rittirsch D, Flierl MA, Ward PA. Harmful molecular mechanisms in sepsis. Nat Rev Immunol 2008; 8:77687; PMID:18802444; http://dx.doi.org/10.1038/ nri2402.

7. Stearns-Kurosawa DJ, Osuchowski MF, Valentine C, Kurosawa S, Remick DG. The pathogenesis of sepsis. Annu Rev Pathol 2011; 6:19-48; PMID:20887193; http://dx.doi.org/10.1146/annurevpathol-011110-130327.
Survival data were analyzed by the $\chi^{2}$ test for individual time points and by the logrank test ${ }^{52}$ for the entire observation period. The Cox proportional hazard survival regression model was used to determine hazard ratios of death. ${ }^{53}$ Unpaired student's t-test was used to compare times to death and TNF $\alpha$ concentrations. Deep $T_{b}$ values were compared across treatments and time points by two-way ANOVA; p-values for the entire duration of response are reported. A difference was considered significant at $\mathrm{p}<0.05$. Results are reported in the format mean \pm SE.

\section{Disclosure of Potential Conflicts of Interest}

N.R.G. is employed by Amgen Inc. A.A.R. has consulted for TRP programs at Amgen, Inc. and several other pharmaceutical companies, and his TRP-related research has been supported by Amgen, Inc. and Abbott Laboratories.

\section{Acknowledgements}

We thank Tatiane B. Nucci and Justin Eales for their help with animal work, Drs. Junwei Hao and Fu-Dong Shi for help with the ELISA assay, and Catherine M. Krall and Julie M. Turko for editing the manuscript. A.G.'s and E.P.'s present address is University of Pécs Medical School, Pécs, Hungary. This research has been supported in part by the National Institutes of Health (grant R01NS41233 to A.A.R.) and Amgen, Inc., (study agreements with A.A.R.). S.P.W. was a Fellow of the National Council for Scientific and Technological Development, Brazil. Author contributions: S.P.W., C.C.C. and A.A.R. designed the study. S.P.W., A.G., E.P. and D.L.O. performed experiments. N.R.G. provided reagents and materials. S.P.W. and A.A.R. analyzed data and wrote the manuscript.
8. Hyde SR, Stith RD, McCallum RE. Mortality and bacteriology of sepsis following cecal ligation and puncture in aged mice. Infect Immun 1990; 58:61924; PMID:2307515.

9. Chorinchath BB, Kong LY, Mao L, McCallum RE Age-associated differences in TNF-alpha and nitric oxide production in endotoxic mice. J Immunol 1996; 156:1525-30; PMID:8568256

10. Tateda K, Matsumoto T, Miyazaki S, Yamaguchi K. Lipopolysaccharide-induced lethality and cytokine production in aged mice. Infect Immun 1996; 64:769-74; PMID:8641780.

11. Turnbull IR, Wlzorek JJ, Osborne D, Hotchkiss RS, Coopersmith CM, Buchman TG. Effects of age on mortality and antibiotic efficacy in cecal ligation and puncture. Shock 2003; 19:310-3; PMID:12688540; http://dx.doi.org/10.1097/00024382-20030400000003 .

12. Caterina MJ, Schumacher MA, Tominaga M, Rosen TA, Levine JD, Julius D. The capsaicin receptor: a heat-activated ion channel in the pain pathway. Nature 1997; 389:816-24; PMID:9349813; http://dx.doi. org/10.1038/39807.

13. Tominaga $M$, Caterina $M J$, Malmberg $A B$, Rosen TA, Gilbert H, Skinner K, et al. The cloned capsaicin receptor integrates multiple pain-producing stimuli. Neuron 1998; 21:531-43; PMID:9768840; http:// dx.doi.org/10.1016/S0896-6273(00)80564-4.

14. Murai M, Tsuji F, Nose M, Seki I, Oki K, Setoguchi C, et al. SA13353 (1-[2-(1-Adamantyl)ethyl]-1-pentyl-3-[3-(4-pyridyl)propyl]urea) inhibits TNF-alpha production through the activation of capsaicin-sensitive afferent neurons mediated via transient receptor potential vanilloid 1 in vivo. Eur J Pharmacol 2008; 588:309-15; PMID:18508045; http://dx.doi. org/10.1016/j.ejphar.2008.04.037.
15. Clark N, Keeble J, Fernandes ES, Starr A, Liang L Sugden D, et al. The transient receptor potential vanilloid 1 (TRPV1) receptor protects against the onset of sepsis after endotoxin. FASEB J 2007; 21:3747-55; PMID:17601984; http://dx.doi.org/10.1096/fj.067460com.

16. Wang Y, Novotny M, Quaiserova-Mocko V, Swain GM, Wang DH. TRPV1-mediated protection against endotoxin-induced hypotension and mortality in rats. Am J Physiol Regul Integr Comp Physiol 2008; 294:1517-23; PMID:18337316; http://dx.doi org/10.1152/ajpregu.00005.2008.

17. Guptill V, Cui X, Khaibullina A, Keller JM, Spornick $\mathrm{N}$, Mannes A, et al. Disruption of the transient receptor potential vannilloid 1 can affect survival, bacterial clearance and cytokine gene expression during murine sepsis. Anesthesiology 2011; 114:1190 9; PMID:21383614; http://dx.doi.org/10.1097/ ALN.0b013e318212515b.

18. Gavva NR, Treanor JJ, Garami A, Fang L, Surapaneni S, Akrami A, et al. Pharmacological blockade of the vanilloid receptor TRPV1 elicits marked hyperthermia in humans. Pain 2008; 136:202-10; PMID:18337008; http://dx.doi.org/10.1016/j.pain.2008.01.024.

19. Garami A, Shimansky YP, Pakai E, Oliveira DL, Gavva NR, Romanovsky AA. Contributions of different modes of TRPV1 activation to TRPV1 antagonistinduced hyperthermia. J Neurosci 2010; 30:143540; PMID:20107070; http://dx.doi.org/10.1523/ JNEUROSCI.5150-09.2010.

20. Romanovsky AA, Almeida MC, Garami A, Steiner AA, Norman MH, Morrison SF, et al. The transient receptor potential vanilloid-1 channel in thermoregulation: a thermosensor it is not. Pharmacol Rev 2009; 61:22861; PMID:19749171; http://dx.doi.org/10.1124/ pr.109.001263. 
21. Steiner AA, Oliveira DL, Roberts JL, Petersen SR, Romanovsky AA. Nicotine administration and withdrawal affect survival in systemic inflammation models. J Appl Physiol 2008; 105:1028-34; PMID:18617624; http://dx.doi.org/10.1152/japplphysiol.90619.2008.

22. Cross AS, Sadoff JC, Kelly N, Bernton E, Gemski P. Pretreatment with recombinant murine tumor necrosis factor alpha/cachectin and murine interleukin 1-alpha protects mice from lethal bacterial infection. J Exp Med 1989; 169:2021-7; PMID:2659724; http://dx.doi. org/10.1084/jem.169.6.2021.

23. Cross A, Asher L, Seguin M, Yuan L, Kelly N, Hammack $\mathrm{C}$, et al. The importance of a lipopolysaccharide-initiated, cytokine-mediated host defense mechanism in mice against extraintestinally invasive Escherichia coli. J Clin Invest 1995; 96:676-86; PMID:7635960; http:// dx.doi.org/10.1172/JCI118110.

24. Vazquez-Torres A, Vallance BA, Bergman MA, Finlay BB, Cookson BT, Jones-Carson J, et al. Toll-like receptor 4 dependence of innate and adaptive immunity to Salmonella: importance of the Kupffer cell network. J Immunol 2004; 172:6202-8; PMID:15128808.

25. Wang H, Liao H, Ochani M, Justiniani M, Lin X, Yang L, et al. Cholinergic agonists inhibit HMGB1 release and improve survival in experimental sepsis. Nat Med 2004; 10:1216-21; PMID:15502843; http://dx.doi. org/10.1038/nm1124.

26. Pavlov VA, Ochani M, Yang LH, Gallowitsch-Puerta $\mathrm{M}$, Ochani K, Lin X, et al. Selective alpha7-nicotinic acetylcholine receptor agonist GTS-21 improves survival in murine endotoxemia and severe sepsis. Crit Care Med 2007; 35:1139-44; PMID:17334244; http:// dx.doi.org/10.1097/01.CCM.0000259381.56526.96.

27. Hofer S, Eisenbach C, Lukic IK, Schneider L, Bode $\mathrm{K}$, Brueckmann M, et al. Pharmacologic cholinesterase inhibition improves survival in experimental sepsis. Crit Care Med 2008; 36:404-8; PMID:18091537; http:// dx.doi.org/10.1097/01.CCM.0B013E31816208B3.

28. Ang SF, Moochhala SM, Bhatia M. Hydrogen sulfide promotes transient receptor potential vanilloid 1-mediated neurogenic inflammation in polymicrobial sepsis. Crit Care Med 2010; 38:619-28; PMID:19851090 http://dx.doi.org/10.1097/CCM.0b013e3181c0df00.

29. Garami A, Pakai E, Oliveira DL, Steiner AA, Wanner SP, Almeida MC, et al. Thermoregulatory phenotype of the Trpv1 knockout mouse: thermoeffector dysbalance with hyperkinesis. J Neurosci 2011; 31:172133; PMID:21289181; http://dx.doi.org/10.1523/ JNEUROSCI.4671-10.2011.

30. Wanner SP, Garami A, Romanovsky AA. Hyperactive when young, hypoactive and overweight when aged connecting the dots in the story about locomotor activity, body mass and aging in Trpv1 knockout mice. Aging (Albany NY) 2011; 3:450-4; PMID:21483038.

31. Steiner AA, Turek VF, Almeida MC, Burmeister JJ, Oliveira DL, Roberts JL, et al. Nonthermal activation of transient receptor potential vanilloid-1 channels in abdominal viscera tonically inhibits autonomic cold-defense effectors. J Neurosci 2007; 27:745968; PMID:17626206; http://dx.doi.org/10.1523/ JNEUROSCI.1483-07.2007.
32. Motter AL, Ahern GP. TRPV1-null mice are protected from diet-induced obesity. FEBS Lett 2008; 582:2257 62; PMID:18503767; http://dx.doi.org/10.1016/j.febslet.2008.05.021

33. Ivanov AI, Kulchitsky VA, Romanovsky AA. Does obesity affect febrile responsiveness? Int J Obes Rela Metab Disord 2001; 25:586-9; PMID:11319666 http://dx.doi.org/10.1038/sj.ijo.0801523.

34. Ivanov AI, Romanovsky AA. Fever responses of Zucker rats with and without fatty mutation of the leptin receptor. Am J Physiol Regul Integr Comp Physio 2002; 282:311-6; PMID:11742853.

35. Ivanov AI, Kulchitsky VA, Romanovsky AA. Role for the cholecystokinin-A receptor in fever: a study of a mutant rat strain and a pharmacological analysis. J Physiol 2003; 547:941-9; PMID:12562931; http:// dx.doi.org/10.1113/jphysiol.2002.033183.

36. Steiner AA, Dogan MD, Ivanov AI, Patel S, Rudaya AY, Jennings DH, et al. A new function of the leptin receptor: mediation of the recovery from lipopolysaccharideinduced hypothermia. FASEB J 2004; 18:1949-51; PMID:15388670.

37. Ivanov AI, Steiner AA, Patel S, Rudaya AY, Romanovsky AA. Albumin is not an irreplaceable carrier for amphipathic mediators of thermoregulatory responses to LPS: compensatory role of $\alpha 1$-acid glycoprotein. Am J Physiol Regul Integr Comp Physiol 2005; 288:872 8; PMID:15576666; http://dx.doi.org/10.1152/ajpregu.00514.2004

38. Miehsler W. Mortality, morbidity and special issues of obese ICU patients. Wien Med Wochensch 2010; 160:124-8; PMID:20364415; http://dx.doi org/10.1007/s10354-010-0767-4.

39. Steiner AA, Romanovsky AA. Leptin: at the crossroads of energy balance and systemic inflammation. Prog Lipid Res 2007; 46:89-107; PMID:17275915; http:// dx.doi.org/10.1016/j.plipres.2006.11.001.

40. Ye J, Keller JN. Regulation of energy metabolism by inflammation: a feedback response in obesity and calorie restriction. Aging (Albany NY) 2010; 2:361-8 PMID:20606248.

41. Wang S, Davis BM, Zwick M, Waxman SG, Albers KM. Reduced thermal sensitivity and Nav1.8 and TRPV1 channel expression in sensory neurons of aged mice. Neurobiol Aging 2006; 27:895-903; PMID:15979214; http://dx.doi.org/10.1016/j.neurobiolaging.2005.04.009.

42. Bergman E, Fundin BT, Ulfhake B. Effects of aging and axotomy on the expression of neurotrophin receptors in primary sensory neurons. Comp Neurol 1999; 410:368-86; PMID:10404406 http://dx.doi.org/10.1002/(SICI) 1096 9861(19990802)410:3<368::AID-CNE2>3.0.CO;2-I.

43. Marik PE, Zaloga GP. The effect of aging on circulating levels of proinflammatory cytokines during septic hock. Norasept II Study Investigators. J Am Geriat Soc 2001; 49:5-9; PMID:11207836; http://dx.doi. org/10.1046/j.1532-5415.2001.49003.x.
44. Stewart L, Grifiss JM, Jarvis GA, Way LW. Elderly patients have more severe biliary infections: influence of complement-killing and induction of TNFalpha production. Surgery 2008; 143:103-12; PMID:18154938; http://dx.doi.org/10.1016/j.surg.2007.06.035.

45. Watanabe E, Hirasawa H, Oda S, Matsuda K, Hatano M, Tokuhisa T. Extremely high interleukin-6 blood levels and outcome in the critically ill are associated with tumor necrosis factor- and interleukin-1-related gene polymorphisms. Crit Care Med 2005; 33:89 97; PMID:15644653; http://dx.doi.org/10.1097/01 CCM.0000150025.79100.7D.

46. Turnbull IR, Clark AT, Stromberg PE, Dixon DJ, Woolsey CA, Davis CG, et al. Effects of aging on the immunopathologic response to sepsis. Crit Care Med 2009; 37:1018-23; PMID:19237912; http://dx.doi. org/10.1097/CCM.0b013e3181968f3a.

47. McConnell KW, Fox AC, Clark AT, Chang NY Dominguez JA, Farris AB, et al. The role of heat shock protein 70 in mediating age-dependent mortality in sepsis. J Immunol 2011; 186:3718-25 PMID:21296977; http://dx.doi.org/10.4049/jimmunol.1003652.

48. Moran MM, McAlexander MA, Biro T, Szallasi A Transient receptor potential channels as therapeutic targets. Nat Rev Drug Discov 2011; 10:601 20; PMID:21804597; http://dx.doi.org/10.1038/ $\operatorname{nrd} 3456$.

49. Norman DC. Fever in the elderly. Clin Infect Dis 2000; 31:148-51; PMID:10913413; http://dx.doi. org/10.1086/313896

50. High KP, Bradley SF, Gravenstein S, Mehr DR, Quagliarello VJ, Richards C, et al. Clinical practice guideline for the evaluation of fever and infection in older adult residents of long-term care facilities: 2008 update by the Infectious Diseases Society of America. J Am Geriatr Soc 2009; 57:375-94; PMID:19278394; http://dx.doi.org/10.1111/j.1532-5415.2009.02175.x.

51. Rudaya AY, Steiner AA, Robbins JR, Dragic AS, Romanovsky AA. Thermoregulatory responses to lipopolysaccharide in the mouse: dependence on the dose and ambient temperature. Am J Physiol Regul Integr Comp Physiol 2005; 289:1244-52; PMID:16081879; http://dx.doi.org/10.1152/ajpregu.00370.2005.

52. Bland JM, Altman DG. The logrank test. BM 2004; 328:1073; PMID:15117797; http://dx.doi. org/10.1136/bmj.328.7447.1073.

53. Lawless JF. Statistical Models and Methods for Lifetime Data. New York: John Wiley \& Sons 1982. 


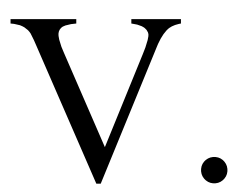




\title{
Thermoregulatory Phenotype of the Trpv1 Knockout Mouse: Thermoeffector Dysbalance with Hyperkinesis
}

\author{
Andras Garami, ${ }^{1}$ Eszter Pakai, ${ }^{1}$ Daniela L. Oliveira, ${ }^{1}$ Alexandre A. Steiner, ${ }^{1}$ Samuel P. Wanner,,${ }^{1,2}$ M. Camila Almeida, ${ }^{1}$ \\ Vladimir A. Lesnikov, ${ }^{3}$ Narender R. Gavva, ${ }^{4}$ and Andrej A. Romanovsky ${ }^{1}$ \\ ${ }^{1}$ Systemic Inflammation Laboratory, Trauma Research, St. Joseph's Hospital and Medical Center, Phoenix, Arizona 85013, ${ }^{2}$ Department of Physiology \\ and Biophysics, Federal University of Minas Gerais, Belo Horizonte, Minas Gerais 31270-901, Brazil, ${ }^{3}$ Fred Hutchinson Cancer Research Center, Seattle, \\ Washington 98109, and ${ }^{4}$ Department of Neuroscience, Amgen, Thousand Oaks, California 91320
}

This study aimed at determining the thermoregulatory phenotype of mice lacking transient receptor potential vanilloid-1 (TRPV1) channels. We used Trpv1 knockout (KO) mice and their genetically unaltered littermates to study diurnal variations in deep body temperature $\left(T_{\mathrm{b}}\right)$ and thermoeffector activities under basal conditions, as well as thermoregulatory responses to severe heat and cold. Only subtle alterations were found in the basal $T_{\mathrm{b}}$ of $\operatorname{Trpv} 1 \mathrm{KO}$ mice or in their $T_{\mathrm{b}}$ responses to thermal challenges. The main thermoregulatory abnormality of $\operatorname{Trpv} 1 \mathrm{KO}$ mice was a different pattern of thermoeffectors used to regulate $T_{\mathrm{b}}$. On the autonomic side, $\operatorname{Trpv} 1 \mathrm{KO}$ mice were hypometabolic (had a lower oxygen consumption) and hypervasoconstricted (had a lower tail skin temperature). In agreement with the enhanced skin vasoconstriction, Trpv1 KO mice had a higher thermoneutral zone. On the behavioral side, Trpv1 KO mice preferred a lower ambient temperature and expressed a higher locomotor activity. Experiments with pharmacological TRPV1 agonists (resiniferatoxin and anandamide) and a TRPV1 antagonist (AMG0347) confirmed that TRPV1 channels located outside the brain tonically inhibit locomotor activity. With age (observed for up to 14 months), the body mass of Trpv1 K0 mice exceeded that of controls, sometimes approaching $60 \mathrm{~g}$. In summary, Trpv1 $\mathrm{KO}$ mice possess a distinct thermoregulatory phenotype, which is coupled with a predisposition to age-associated overweight and includes hypometabolism, enhanced skin vasoconstriction, decreased thermopreferendum, and hyperkinesis. The latter may be one of the primary deficiencies in Trpv1 K0 mice. We propose that TRPV1-mediated signals from the periphery tonically suppress the general locomotor activity.

\section{Introduction}

The development of new pharmacological and genetic tools to work with the transient receptor potential vanilloid-1 (TRPV1) channel has caused a surge of studies aimed at identifying the roles of this channel in various functions, including the regulation of body temperature $\left(T_{\mathrm{b}}\right)$. Studies using pharmacological tools have revealed clear and highly reproducible effects on $T_{\mathrm{b}}$ : TRPV1 agonists cause hypothermia, whereas TRPV1 antagonists cause hyperthermia (for reviews, see Gavva, 2008; Romanovsky et al., 2009). In contrast to the unequivocal results obtained with pharmacological tools, studies in genetically modified animals have failed to reveal a clear thermoregulatory phenotype (Szelé-

Received Sept. 6, 2010; revised 0ct. 25, 2010; accepted Nov. 23, 2010.

This research has been supported by Amgen (study agreements with A.A.R.), the National Institutes of Health (Grant R01NS41233 to A.A.R.), and the National Council for Scientific and Technological Development, Brazil (Visiting Ph.D. Student Fellowship to S.P.W.). We thank Tatiane N. Cooper and Dr. Yury P. Shimansky for the help with the densitometry analysis of thermograms and with statistical analyses, respectively.

N.R.G. is employed by Amgen, Inc. A.A.R. has consulted for Amgen, Inc., and TRP programs at several pharmaceutical companies, and his research has been supported by Amgen, Inc.

Correspondence should be addressed to Andrej A. Romanovsky, Systemic Inflammation Laboratory, Trauma Research, St. Joseph's Hospital and Medical Center, 350 West Thomas Road, Phoenix, AZ 85013. E-mail: aromano@chw.edu.

A. A. Steiner's present address: Department of Pharmaceutical Sciences, Albany College of Pharmacy and Health Sciences, Albany, NY 12208.

M. C. Almeida's present address: Federal University of ABC, Santo Andre, Sao Paulo 09210-170, Brazil.

DOI:10.1523/JNEUROSCI.4671-10.2011

Copyright $\odot 2011$ the authors $\quad 0270-6474 / 11 / 311721-13 \$ 15.00 / 0$ nyi et al., 2004; Iida et al., 2005; Christoph et al., 2008; Motter and Ahern, 2008). Szelényi et al. (2004) have reported that Trpv1 knockout (KO) mice have an exaggerated magnitude of daynight fluctuations in $T_{\mathrm{b}}$, whereas Iida et al. (2005) have not seen such an exaggeration. Motter and Ahern (2008) have found that Trpv1 KO mice have an enhanced capacity for cold-induced thermogenesis, whereas Iida et al. (2005) have not seen such an enhancement. Knowing that thermoregulatory responses in mice are notoriously difficult to study (Rudaya et al., 2005), we reexamined the ability of Trpv1 KO mice to regulate $T_{\mathrm{b}}$. Because thermoeffectors are regulated independently (Romanovsky, 2007; Morrison et al., 2008; McAllen et al., 2010), we studied several autonomic and behavioral effector mechanisms. Because ambient temperature $\left(T_{\mathrm{a}}\right)$ affects many thermoregulatory responses and often determines the pattern of effectors used to regulate deep $T_{\mathrm{b}}$ (Romanovsky et al., 2002), the present study was conducted under tightly controlled thermal conditions. Furthermore, it was verified in each experimental setup used whether the thermal conditions were neutral [i.e., within the thermoneutral zone (TNZ)], subneutral, or supraneutral.

Initially, the primary goal of this study was to determine a thermoregulatory phenotype of the Trpv1 KO mouse. After we found that the absence of the Trpv1 gene was accompanied by an increased locomotor activity, which is considered to be a thermoregulatory effector in small rodents (Mount and Willmott, 1967; Brown et al., 1991; Weinert and Waterhouse, 1998), we focused 
the second part of the study on this novel finding. In pharmacological experiments, we used TRPV1 agonists and a TRPV1 antagonist to investigate the potential involvement of TRPV1 channels in the regulation of locomotor activity. Based on experiments with Trpv1 KO mice and with pharmacological tools, we propose that tonic activation of peripheral TRPV1 channels suppresses the general locomotor activity. Even though TRPV1 channels are unlikely to be those long-sought thermosensors that respond to skin temperature $\left(T_{\mathrm{sk}}\right)$ or deep $T_{\mathrm{b}}$ signals to regulate thermoeffector activity (Romanovsky et al., 2009), the tonic, nonthermal activation of TRPV1 channels does modulate several effector responses (Steiner et al., 2007), perhaps in a state-specific manner (Kanizsai et al., 2009). It is likely, therefore, that new mechanisms relevant to the TRPV1-dependent modulation of $T_{\mathrm{b}}$ remain to be discovered.

\section{Materials and Methods}

Animals

Physiological experiments were conducted in 293 adult mice of both sexes. Of these, 269 C57BL/6x129 mice were obtained from the Amgen colony at Charles River Laboratories. These mice had the Trpv1 gene either present $\left(\operatorname{Trp} v 1^{+/+}\right)$or missing $\left(\operatorname{Trp} v 1^{-/-}\right)$due to a targeted null mutation (Caterina et al., 2000). They were produced by breeding $\operatorname{Trpv1} 1^{+/-}$males and females and genotyping their offspring; the breeding and genotyping were performed by Charles River Laboratories at their facilities. Twenty-four additional $\operatorname{Trpv} 1^{+/+}$mice of a different genotype, C57BL/6, were purchased from Charles River Laboratories. Except for the body mass comparison study, where the mice were observed for an extended period of time (from the age of 4 weeks to the age of 56 weeks), all experiments were performed in male mice with body mass of $\sim 30 \mathrm{~g}$ (age of $\sim 17$ weeks). The mice were housed in standard "shoebox" cages kept in a Maxi-Miser ventilated rack (Thoren Caging Systems) at a $T_{\mathrm{a}}$ of $27^{\circ} \mathrm{C}$. The room was on a $12 \mathrm{~h} \mathrm{light/dark} \mathrm{cycle} \mathrm{(lights} \mathrm{on} \mathrm{at} \mathrm{6:00} \mathrm{A.M.).}$ Tap water and standard (5.0\% fat) rodent chow (Laboratory rodent diet 5001; Labdiet) were available ad libitum. The body mass of mice was measured regularly with a portable digital scale (model VI-200; Acculab). At the end of the study, we confirmed the absence or presence of functional TRPV1 channels in the $\mathrm{KO}$ and control mice, respectively. For this, $24 \operatorname{Trpv1}^{-/-}$and $24 \operatorname{Trpv} 1^{+/+}$C57BL/6x129 mice were randomly selected from the study population, food deprived for $36 \mathrm{~h}$, and then exposed to an excessive amount of habanero chili (Capsicum chinense). Habanero is one of the hottest chili peppers with a reported pungency of 100,000-300,000 Scoville units (Berkley and Jacobson, 1992). For a 20 min period, eating events were counted by an observer blind to the mice's genotype. Trpv1 KO mice eagerly consumed the peppers, whereas the controls did not $(p<0.0001)$ (Fig. 1).

For all experiments, the mice were extensively handled ( 8 daily sessions, $5 \mathrm{~min}$ each) and then habituated to experimental setups as follows. A mouse designated for an experiment in the telemetry, thermocouple, or respirometry setup (see below, Experimental setups), was adapted to staying in either a Plexiglas enclosure or a wire-mesh confiner (8 training sessions, $1-4 \mathrm{~h}$ each). The enclosure, which had a square $(15 \times 15 \mathrm{~cm})$ base and 25-cm-high walls, did not limit the animal's movement; it was used for experiments in the telemetry setup. The cylindrical confiner (length, $10 \mathrm{~cm}$; diameter, $3 \mathrm{~cm}$ ) prevented the animal from turning around but allowed for some back-and-forth movements; it was used in the thermocouple and respirometry setups. A mouse designated for an experiment in the thermogradient setup was adapted to staying in the channels of the thermogradient apparatus ( 7 sessions, 3-24 h each). During long training sessions $(>12 \mathrm{~h})$ the animal had unlimited access to food and water. All protocols were approved by the St. Joseph's Hospital and Medical Center Animal Care and Use Committee.

\section{Surgical preparations}

Anesthesia and perioperative care. Surgeries were performed under ketamine-xylazine-acepromazine (81.7, 9.3 , and $1.2 \mathrm{mg} / \mathrm{kg}$, i.p.) anesthesia and antibiotic protection (enrofloxacin, $1.1 \mathrm{mg} / \mathrm{kg}$, s.c.). During surgery, a mouse was heated with a Deltaphase isothermal pad (Braintree

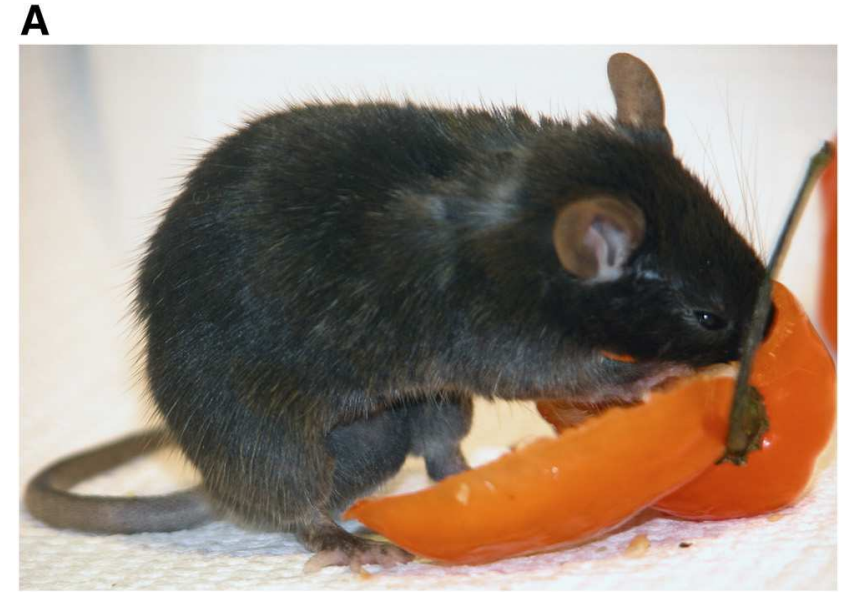

B

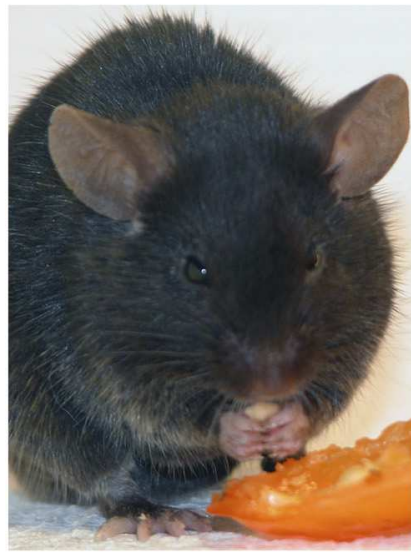

C

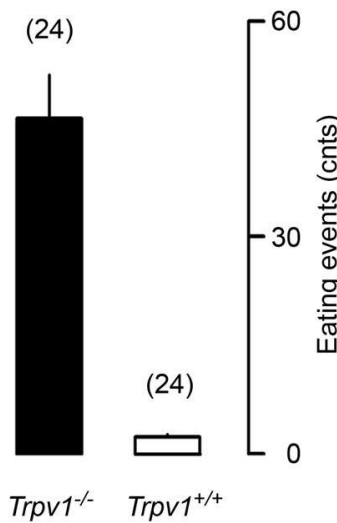

Figure 1. Behavioral verification of TRPV1 deficiency in Trpv1 K0 mice. Trpv1 K0 mice are insensitive to capsaicin and, when food deprived, eagerly consume habanero chili $(\boldsymbol{A})$, including the hottest parts, such as the septa and seeds $(\boldsymbol{B})$. The mean number of pepper-eating events in $\operatorname{Trpv} 1^{-/-}$versus $\operatorname{Trpv} 1^{+/+}$mice over a 20 min period is also shown (C). Here and in Figures 2 , 3 , and $5-10$, numbers in parentheses are the numbers of animals in the corresponding groups.

Scientific) and periodically (every $5 \mathrm{~min}$ ) ventilated with oxygen through a custom-made mask. The isothermal pad was placed either under a surgery board (Plas-Labs) or on the base of a stereotaxic apparatus. For immediate postoperative recovery, the mouse was placed in a climatic chamber set to a $T_{\mathrm{a}}$ of $31.0^{\circ} \mathrm{C}$. Experiments were performed $5-7 \mathrm{~d}$ after surgery.

Implantation of temperature-measuring devices. If the mouse was designated for an experiment in the telemetry setup, it was implanted with a miniature telemetry transmitter (G2 E-Mitter series; Mini Mitter) to record abdominal temperature $\left(T_{\mathrm{ab}}\right.$, a measure of deep $\left.T_{\mathrm{b}}\right)$ and gross locomotor activity. If the mouse was designated for an experiment in the thermogradient setup, it was implanted with a miniature temperature datalogger (Subcue Dataloggers) to record and store $T_{\mathrm{ab}}$ data. Either device was inserted into the peritoneal cavity via midline laparotomy and fixed to the lateral abdominal wall (right side) with a suture. The surgical wound was sutured in layers.

Intraperitoneal catheterization. For the intraperitoneal administration of drugs, a silicone catheter filled with pyrogen-free saline was inserted into the peritoneal cavity through the same opening in the abdominal wall that was used to insert a temperature-measuring device, and the internal end of the catheter was attached with a suture to the lateral abdominal wall on the left side. The free end of the catheter was knotted, tunneled under the skin to the nape, and exteriorized. The catheter was flushed with saline on the day after surgery and every other day thereafter.

Intracerebroventricular cannulation. For the intracerebroventricular drug administration, a 22 ga steel guide cannula (Plastics One) was im- 
Table 1. Experimental setups

\begin{tabular}{|c|c|c|c|}
\hline \multirow[b]{2}{*}{ Setup } & \multicolumn{2}{|c|}{ Measures of deep body temperature $\left(T_{\mathrm{b}}\right)$ and thermoeffector activities studied } & \multirow[b]{2}{*}{ Neutral ambient temperature $\left(T_{\mathrm{a}},{ }^{\circ} \mathrm{C}\right)^{a}$} \\
\hline & $T_{\mathrm{b}}$ & Thermoeffector activities & \\
\hline Telemetry & $\operatorname{Abdominal}\left(T_{\mathrm{ab}}\right)$ & $\begin{array}{l}\text { Gross locomotor activity (measured as frequency of probe movement) } \\
\text { Tail skin vasomotion (measured as tail skin temperature, } T_{\text {sk, }} \text {, by thermocouple thermometry }\end{array}$ & 31.0 \\
\hline Thermocouple & Colonic $\left(T_{c}\right)$ & or as radiant $T_{\text {sk }}$ by infrared thermography) & $32.0^{b}$ \\
\hline Respirometry & $T_{\mathrm{c}}$ & Thermogenesis (measured as rate of oxygen consumption, $V_{0_{2}}$ ), tail skin vasomotion $\left(T_{\mathrm{sk}}\right)$ & $33.0^{c}$ \\
\hline Thermogradient & $T_{\mathrm{ab}}$ & Selection of preferred $T_{\mathrm{a}}$, locomotor activity (measured as velocity of longitudinal locomotion) & $27.0^{d}$ \\
\hline
\end{tabular}

${ }^{a}$ For each setup, the table lists one $T_{\mathrm{a}}$ that has been shown to be within the TNZ for $\operatorname{Trpv} 1^{+/+} \mathrm{CB} 57 \mathrm{BL} / 6$ mice in that setup. ${ }^{b} \mathrm{For}$ the lower and upper limits of the TNZ for Trpv1 ${ }^{-/-}$and $\operatorname{Trpv} 1^{+/+} \mathrm{CB} 57 \mathrm{BL} / 6 \mathrm{x} 129$ mice in the thermocouple

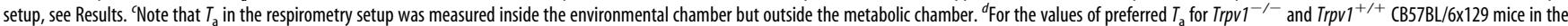
thermogradient setup, see Results.

planted into the right lateral brain ventricle using the stereotaxic atlas by Lesnikov and Tsvetkova (1985) and their original stereotaxic apparatus. The head of a mouse was fixed in the stereotaxic apparatus; the scalp was incised over the sagittal suture; the periosteum was excised; the bone surface was cleaned; and three supporting microscrews were driven into the skull. The position of the head of the mouse was then adjusted so that bregma was located $0.5 \mathrm{~mm}$ higher than lambda, and a hole was drilled $0.5 \mathrm{~mm}$ posterior to bregma and $1.0 \mathrm{~mm}$ right of the sagittal suture. The guide cannula with a 28 ga dummy injector inside was inserted into the lateral ventricle by lowering the tip of the injector (which protruded the cannula by $1.0 \mathrm{~mm}$ ) $3.0 \mathrm{~mm}$ below the skull surface. The cannula was affixed to the supporting microscrews with acrylic cement. At the end of the experiments, a correct placement of each cannula was confirmed by anesthetizing the animal, infusing $3 \mu \mathrm{l}$ of an aqueous solution of methylene blue $(6 \mathrm{mg} / \mathrm{ml})$ through the cannula, removing the brain, and examining coronal sections macroscopically for the presence of the dye in the ventricular system.

\section{Experimental setups}

Experiments were conducted in one of the following setups: the telemetric thermometry ("telemetry") setup, the thermocouple thermometry ("thermocouple") setup, the thermocouple thermometry with respirometry ("respirometry") setup, and the thermogradient setup (Table 1). All experiments, unless specified otherwise, were conducted under thermoneutral conditions. Because the same animal has a different TNZ in each setup (Romanovsky et al., 2002), the setups were used at different $T_{\mathrm{a}} \mathrm{s}$ (Table 1). In these setups, we measured deep $T_{\mathrm{b}}$ [as either $T_{\mathrm{ab}}$ or colonic temperature $\left.\left(T_{c}\right)\right]$ and four thermoeffector activities, two autonomic and two behavioral (Table 1). On the autonomic side, we measured tail skin vasomotion (assessed by tail skin temperature, $T_{\mathrm{sk}}$ ) and the rate of oxygen consumption $\left(V_{\mathrm{O}_{2}}\right)$. On the behavioral side, we measured the preferred $T_{\mathrm{a}}$ and general locomotor activity. The latter has been shown repeatedly to play an important thermoregulatory role in small rodents such as rats and mice (Mount and Willmott, 1967; Brown et al., 1991; Weinert and Waterhouse, 1998) and is now widely viewed as a thermoregulatory effector in these species (Kanizsai et al., 2009; Hunt et al., 2010; Szentirmai et al., 2010). The locomotor activity was measured in this study either as the velocity of longitudinal movement in the thermogradient apparatus or as the frequency of changes of the position or orientation of the implanted telemetry probe in the telemetry setup.

Telemetry setup. Telemetry receivers (model ER-4000; Mini Mitter) were positioned inside a climatic chamber (model 3940; Forma Scientific). The home cage of each mouse was placed on top of a receiver; a Plexiglas enclosure was placed inside the cage; and the mouse was left in the enclosure. The mouse was preimplanted with a telemetry transmitter (to measure $T_{\mathrm{ab}}$ and locomotor activity). In a subset of mice, the transmitter was implanted in combination with either an intraperitoneal catheter or an intracerebroventricular cannula for drug administration. If the mouse had an intraperitoneal catheter, the catheter was extended with a length of polyethylene-50 tubing filled with a drug of interest. This extension was passed through a wall port of the climatic chamber and connected to a syringe placed in an infusion pump (model KDS 220; KD Scientific). Whereas most experiments in this setup were conducted at a neutral $T_{\mathrm{a}}$ of $31.0^{\circ} \mathrm{C}$ (Table 1 ), a subneutral $T_{\mathrm{a}}$ of $26.0^{\circ} \mathrm{C}$ was used when the expected experimental outcome was a decrease in $T_{\mathrm{b}}$, e.g., in experiments with administration of TRPV1 agonists (Hori, 1984; Romanovsky et al., 2009).
We had a concern that measuring locomotor activity with G2 telemetry probes might overestimate any meaningful locomotion, because this method picks up changes not only in the position of the probe relative to the receiver, but also in the probe orientation. Hence, there was a possibility that breathing movements, intestinal peristalsis, yawning, coughing, shivering, and similar activities may contaminate the results or even mask any changes in true whole-body locomotion. To address this concern, we conducted a separate series of experiments. We mounted a motion sensor (Infrared Cage Top Motion Sensor; Mini Mitter), which works with Mini Mitter hardware and software, on each Plexiglas enclosure and simultaneously recorded locomotor activity with the implanted G2 probes and with the external motion sensors. The external sensorbased method is completely insensitive to any probe movement inside the peritoneal cavity, and requires a true body movement to register an activity event. Two groups of Trpv1 $1^{+/+}$C57BL/6x129 and two groups of $\operatorname{Trpv1} 1^{+/+} \mathrm{C} 57 \mathrm{BL} / 6$ mice were injected intraperitoneally with either resiniferatoxin (RTX) or anandamide (AEA), or with their vehicle, as described below. In all four groups, the activity curves obtained by the two methods looked nearly identical (see Notes). When the obtained activity curves were analyzed by two-way ANOVA (following a linear unit transformation), no significant differences were found in any group. These experiments confirm that the telemetric activity measure is a reliable indicator of locomotor activity.

Thermocouple setup. A mouse equipped with copper-constantan thermocouples (Omega Engineering) to measure $T_{\mathrm{c}}$ and $T_{\mathrm{sk}}$ was placed in a confiner. The colonic thermocouple was inserted $2 \mathrm{~cm}$ beyond the anal sphincter and fixed to the base of the tail with a loop of adhesive tape. The skin thermocouple was positioned on the lateral surface of the tail (at the boundary of the proximal and middle thirds) and insulated from the environment with tape. The thermocouples were plugged into a data logger (Cole-Parmer). The confiner with the mouse inside was transferred to a climatic chamber. Whereas most experiments in this setup were conducted at a neutral $T_{\mathrm{a}}$ of $32.0^{\circ} \mathrm{C}$ (Table 1), this setup was also used for heat-exposure experiments, in which $T_{\mathrm{a}}$ was raised from 31.0 to $39.0^{\circ} \mathrm{C}$ over $\sim 30 \mathrm{~min}$ and then maintained at $39.0^{\circ} \mathrm{C}$.

In a separate series of experiments, thermocouples were not used, and radiant $T_{\mathrm{sk}}$ was monitored by infrared thermography with a ThermoVision A20M camera (FLIR Systems) to assess the vasomotor tone of the tail skin and to determine the TNZ (Romanovsky et al., 2002). The ThermoVision camera was positioned above a group of confined mice inside the climatic chamber set to $T_{\mathrm{a}}$ randomly selected from the following list: $30.0,30.5,31.5,32.0,32.5,33.0,33.5$, or $35.0^{\circ} \mathrm{C}$. The mice were kept at this $T_{\mathrm{a}}$ for $\sim 2 \mathrm{~h}$, and infrared thermograms were taken. Thereafter, $T_{\mathrm{a}}$ was changed in a stepwise fashion to another value from the same list and maintained at this new level for $\sim 2 \mathrm{~h}$. Infrared thermograms of the mice were taken again, and a new $T_{\mathrm{a}}$ was chosen. Usually, three to four temperatures were studied in the same experiment.

Respirometry setup. Each mouse was equipped with thermocouples as for experiments in the thermocouple setup. Then each mouse in its confiner was placed inside a cylindrical Plexiglas chamber (Sable Systems), which was sealed. The Plexiglas chambers with mice inside were kept in a climatic chamber and continuously ventilated. The airflow was maintained at $600 \mathrm{ml} / \mathrm{min}$ with the help of a mass flow controller (Sierra Instruments). The air leaving each chamber was automatically sampled, dried, and passed through an oxygen analyzer (Sable Systems). The rate 
of $V_{\mathrm{O}_{2}}$ was calculated by comparing the oxygen fraction in the air exiting the chamber occupied by a mouse to the oxygen fraction in the air exiting an empty chamber (see below, Data processing and analysis). Whereas most experiments in this setup were conducted at a neutral $T_{\mathrm{a}}$ of $33.0^{\circ} \mathrm{C}$ (Table 1 ), this setup was also used for exposing mice to cold. Cooling was performed by decreasing $T_{\mathrm{a}}$ in the climatic chamber from $33.0^{\circ} \mathrm{C}$ to $5.0^{\circ} \mathrm{C}$ over $150 \mathrm{~min}$.

Thermogradient setup. The thermogradient apparatus used has been described in detail previously (Almeida et al., 2006). The apparatus consisted of six 200-cm-long aluminum channels. Each channel had a second (inner) stainless-steel grid floor and an acrylic double-wall lid at the top. At each end, all channels shared a common aluminum wall, which separated the channels from a large tank. The tank at the "warm" end of the channels was filled with water warmed with two electric heating units (PolyScience) to maintain $T_{\mathrm{a}}$ inside the channels at this end at $30.0^{\circ} \mathrm{C}$. The tank at the "cold" end was constantly perfused with $10 \%$ ethylene glycol by an external-circulation cooling/heating pump (PolyScience) to maintain $T_{\mathrm{a}}$ inside the channels at this end at $20.0^{\circ} \mathrm{C}$. In this setting, all channels had a common, nearly linear longitudinal $T_{\mathrm{a}}$ gradient of $0.05^{\circ} \mathrm{C} /$ $\mathrm{cm}$. The position of a mouse in a channel of the thermogradient apparatus was monitored with 56 evenly spaced $(3.5 \mathrm{~cm})$ infrared emitterreceiver pairs, which formed transversal infrared beams. This system also allowed us to directly measure the longitudinal locomotor activity. By knowing how the position of an animal changed in time (as the animal crossed the beams), we knew the velocity of the longitudinal movement (distance traveled divided by time).

\section{Drugs and drug administration}

RTX, a highly potent TRPV1 agonist, was purchased from SigmaAldrich. An ethanolic stock solution of RTX $(200 \mu \mathrm{g} / \mathrm{ml})$ was prepared, aliquoted, and stored at $-80^{\circ} \mathrm{C}$. On the day of the experiment, the stock was diluted with ethanol and saline to achieve a final concentration of RTX of 6,60 , or $150 \mathrm{ng} / \mathrm{ml}$ and a final concentration of ethanol of $10 \%$. For the intraperitoneal administration, this working solution (or the vehicle) was injected acutely, as a bolus ( $3.3 \mathrm{ml} / \mathrm{kg}$ ), using a 26 ga needle. For the intracerebroventricular administration, the same working solution of RTX (or the vehicle) was infused $(1.5 \mu \mathrm{l} / \mathrm{min}$ ) over a $2 \mathrm{~min}$ period. At these infusion volumes and rates, even much higher concentrations of ethanol (50\%) do not cause behavioral responses or changes in $T_{\mathrm{b}}$ (Steiner et al., 2007). For the infusion, the dummy injector (mandrin) was removed from the preimplanted guide cannula and replaced with a 28 ga injector needle (Plastics One) connected to a $25 \mu \mathrm{l}$ Hamilton syringe by a polyethylene- 50 extension. The injector needle protruded $1.0 \mathrm{~mm}$ beyond the tip of the guide cannula. At the end of the infusion, the injector was held in place for an additional $1 \mathrm{~min}$ and then removed and replaced with the mandrin.

AEA (arachidonoyl $N$-ethanolamide), purchased from Tocris Bioscience, was used in the present study as a TRPV1 agonist (Zygmunt et al., 1999; Smart et al., 2000). An ethanolic solution of AEA ( $5 \mathrm{mg} / \mathrm{ml})$ was stored at $-20^{\circ} \mathrm{C}$. On the day of the experiment, the stock was diluted with ethanol and saline to achieve a final concentration of AEA of $0.5 \mathrm{mg} / \mathrm{ml}$ in $10 \%$ ethanol. This working solution of AEA $(15 \mathrm{mg} / \mathrm{kg})$ or the vehicle ( $10 \%$ ethanol) was injected intraperitoneally as a bolus at a total volume of 30 $\mathrm{ml} / \mathrm{kg}$. Because one of the expected outcomes of experiments with TRPV1 agonists was a decrease in the gross locomotor activity (Crawley et al., 1993; Fride and Mechoulam, 1993; Smith et al., 1994; Di Marzo et al., 2001; Wiley et al., 2006), RTX and AEA (or their vehicles) were administered acutely by briefly restricting the animal, pricking it with a needle, bolus injecting the drug, and then releasing the animal. In this paradigm, the handling and needle pricking associated with drug administration elevate the low, nearzero activity typical for habituated mice during the light (inactive) phase.

AMG0347, a highly potent and selective TRPV1 antagonist (Steiner et al., 2007), was provided by Amgen. Aliquots of an ethanolic solution of AMG0347 $(3.5 \mathrm{mg} / \mathrm{ml})$ were stored at $-80^{\circ} \mathrm{C}$. On the day of the experiment, the stock solution was diluted with ethanol and saline to achieve a final concentration of AMG0347 of $15 \mu \mathrm{g} / \mathrm{ml}$ in 50\% ethanol. AMG0347 $(50 \mu \mathrm{g} / \mathrm{kg})$ or its vehicle was administered intraperitoneally as a bolus $(3.3 \mathrm{ml} / \mathrm{kg})$ via a preimplanted catheter. The expected outcome in experiments with AMG0347 was an increase in the locomotor activity. To avoid stress hyperkinesis in these experiments, we used a nonstressful method of intraperitoneal drug administration via a preimplanted cannula.

\section{Data processing and analysis}

The rate of $V_{\mathrm{O}_{2}}$ was calculated by comparing the oxygen fraction in the air exiting the chamber occupied by a mouse $(F)$ to the oxygen fraction in the air exiting an empty chamber $\left(F_{0}\right)$ :

$$
V_{\mathrm{O}_{2}}=\frac{A\left(F_{0}-F\right)}{M-F_{0} M(1+Q)},
$$

where $A$ was air flow, $Q$ was the respiratory quotient (considered to be 0.71 ), and $M$ was the animal's mass (Steiner et al., 2007).

All data collected as time series were compared across genotypes and time points by two-way ANOVA with a Fisher LSD post hoc test, as appropriate. These included the data on deep $T_{\mathrm{b}}$ (whether $T_{\mathrm{ab}}$ or $T_{\mathrm{c}}$ ), measures of thermoeffector activities (i.e., tail $T_{\mathrm{sk}}, V_{\mathrm{O}_{2}}$, preferred $T_{\mathrm{a}}$, and gross locomotor activity), and body mass. For a statistical analysis of the tail images on the infrared thermograms obtained, the optical density was determined for each image by using the VisionWorks 6.5.2 image acquisition and analysis software (Ultra-Violet Products). The optical density values were also compared by two-way ANOVA across genotypes and $T_{\mathrm{a}}$ s. All data collected as single-point measurements (chili pepper eating events) or presented as a single number (light-phase mean, darkphase mean, and daily mean values of $T_{\mathrm{b}}$ measures and those of effector activity measures) were compared between the genotypes by Student's $t$ test. All analyses were performed using Statistica AXA 8.0 (Statsoft). The data are reported as means \pm SE.

\section{Results}

\section{Phase-specific changes in deep $T_{\mathrm{b}}$ of $\operatorname{Trpv} 1 \mathrm{KO}$ mice}

To identify a thermoregulatory phenotype of the Trpv1 KO mouse, we studied circadian fluctuations in deep $T_{\mathrm{b}}$ and thermoeffector activities of a large number of $\operatorname{Tr} p v 1^{-/-}$and $\operatorname{Tr} p v 1^{+/+}$ mice in three experimental setups: thermogradient (Fig. $2 A$ ), telemetry (Fig. $2 B$ ), and respirometry (Fig. $2 C$ ). In all three setups, the light-phase mean deep $T_{\mathrm{b}}$ of $\operatorname{Trp} v 1 \mathrm{KO}$ mice was slightly (by $0.2-0.7^{\circ} \mathrm{C}$ ) but significantly lower than that of the controls (Fig. $2 A-C)$. A similar difference in $T_{\mathrm{b}}$ between the $\mathrm{KO}$ and control mice was found under basal conditions (before any drug administration) in several other experiments in this study (Table 2). During the dark (active) phase, however, the intergenotype $T_{\mathrm{b}}$ difference was attenuated (respirometry setup) (Fig. 2C) or completely disappeared (telemetry setup) (Fig. $2 B$ ). In the thermogradient setup (Fig. $2 \mathrm{~A}$ ), this difference became reversed, that is, the mean dark-phase $T_{\mathrm{ab}}$ of the $\mathrm{KO}$ mice was $0.5^{\circ} \mathrm{C}$ higher than that of the controls $(p<0.05)$. As the result of such biphasic dynamics, the daily mean $T_{\mathrm{b}}$ of $\operatorname{Tr} p v 1 \mathrm{KO}$ mice was either similar to or slightly lower than that of the controls in all setups (Fig. $2 A-C)$. The magnitude of day-night changes in $T_{\mathrm{b}}$ of the Trpv1 $\mathrm{KO}$ mice was increased compared to that of the controls in the thermogradient setup $\left(0.8 \pm 0.2\right.$ vs $\left.0.2 \pm 0.1^{\circ} \mathrm{C} ; p<0.05\right)$ and in the telemetry setup $\left(1.0 \pm 0.1\right.$ vs $\left.0.5 \pm 0.1^{\circ} \mathrm{C} ; p<0.01\right)$. It also had a tendency to be increased in the respirometry setup $(0.5 \pm$ 0.1 vs $0.2 \pm 0.2^{\circ} \mathrm{C} ; p<0.1$ ). In summary, our results agree with studies by others (Szelényi et al., 2004; Iida et al., 2005) showing that Trpv1 KO mice have only slight alterations in the level at which their deep $T_{\mathrm{b}}$ is regulated throughout the day, as compared to control mice. Furthermore, these alterations are phase specific and, to some extent, depend on the experimental setup.

\section{Trpv1 KO mice exhibit a distinct thermoeffector pattern}

What differed drastically between the two genotypes in our study was not the level of $T_{\mathrm{b}}$, but the pattern of behavioral and autonomic effectors used to regulate $T_{\mathrm{b}}$. We studied two behavioral and two autonomic effectors (Table 1). Behavioral effector activities included the "classic" selection of preferred $T_{\mathrm{a}}$ and the gross locomotor activity (Fig. 3A). The autonomic effector activities 


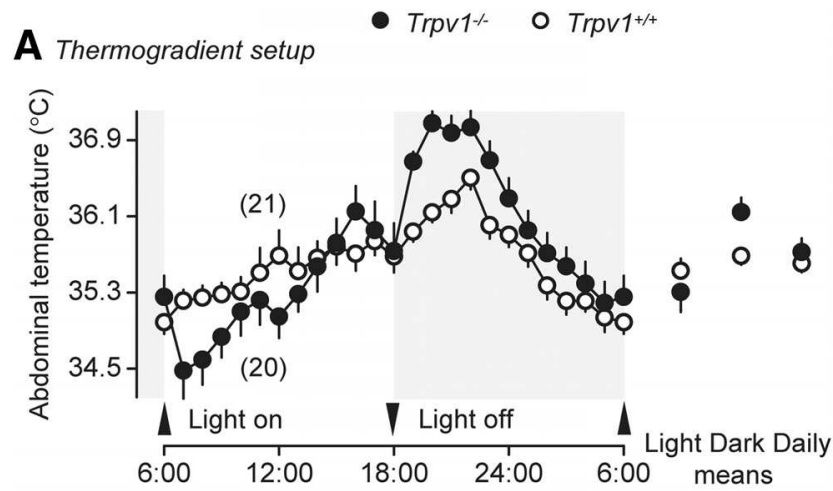

B Telemetry setup

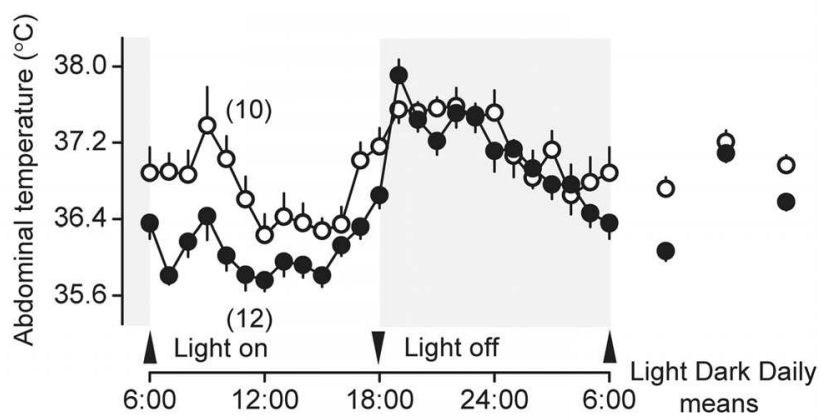

C Respirometry setup

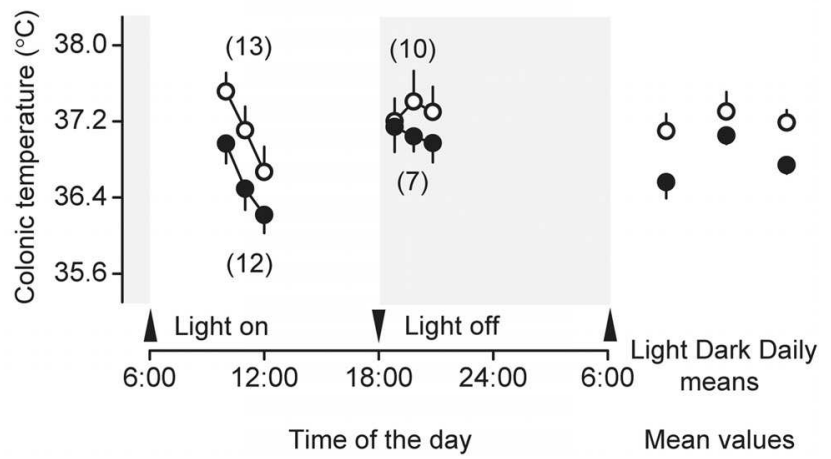

Figure 2. During the inactive (light) phase, Irpv $1 \mathrm{~K} 0$ mice have a lower deep $T_{\mathrm{b}}$ than their genetically unaltered counterparts in the thermogradient $(\boldsymbol{A})$, telemetry $(\boldsymbol{B})$, and respirometry $(\boldsymbol{C})$ setups.

Table 2. Basal deep body temperature ( 30 min mean $\pm \mathrm{SE}$ ) of $\operatorname{Trpv} 1^{+/+}$and $\operatorname{Trpv1} 1^{-/-}$mice in different experimental setups during the light phase

\begin{tabular}{llll}
\hline & $\begin{array}{l}\text { Ambient } \\
\text { Experimental setup }\end{array}$ & \multicolumn{2}{l}{ Basal body temperature $\left({ }^{\circ} \mathrm{C}\right), n$} \\
\cline { 3 - 4 } & temperature $\left({ }^{\circ} \mathrm{C}\right)$ & $\operatorname{Trpv} 1^{+/+}$ & $\operatorname{Trpv}^{-/-}$ \\
\hline Telemetry & 31.0 & $36.7 \pm 0.1$ & $36.3 \pm 0.1^{a}$ \\
& 28.0 & $n=32$ & $n=35$ \\
& & $36.4 \pm 0.1$ & $36.1 \pm 0.1^{b}$ \\
Thermocouple & 32.0 & $n=14$ & $n=14$ \\
Respirometry & 33.0 & $37.1 \pm 0.3$ & $37.3 \pm 0.2$ \\
& & $n=6$ & $n=6$ \\
Thermogradient & $20.0-30.0$ & $36.8 \pm 0.2$ & $36.3 \pm 0.2^{a}$ \\
& & $n=13$ & $n=12$ \\
& & $35.8 \pm 0.3$ & $35.0 \pm 0.2^{a}$ \\
& & $n=21$ & $n=20$ \\
\hline
\end{tabular}

${ }^{a} p<0.05 ;{ }^{b} p<0.1$.

included thermogenesis (measured as $V_{\mathrm{O}_{2}}$ ) and tail skin vasomotor tone (measured as $T_{\text {sk }}$ ) (Fig. $3 B$ ). All four effector activities were reproducibly altered in the setups studied. On the behavioral side, $\operatorname{Tr} p v 1 \mathrm{KO}$ mice selected a lower $T_{\mathrm{a}}$ than their $\operatorname{Tr} p v 1^{+/+}$
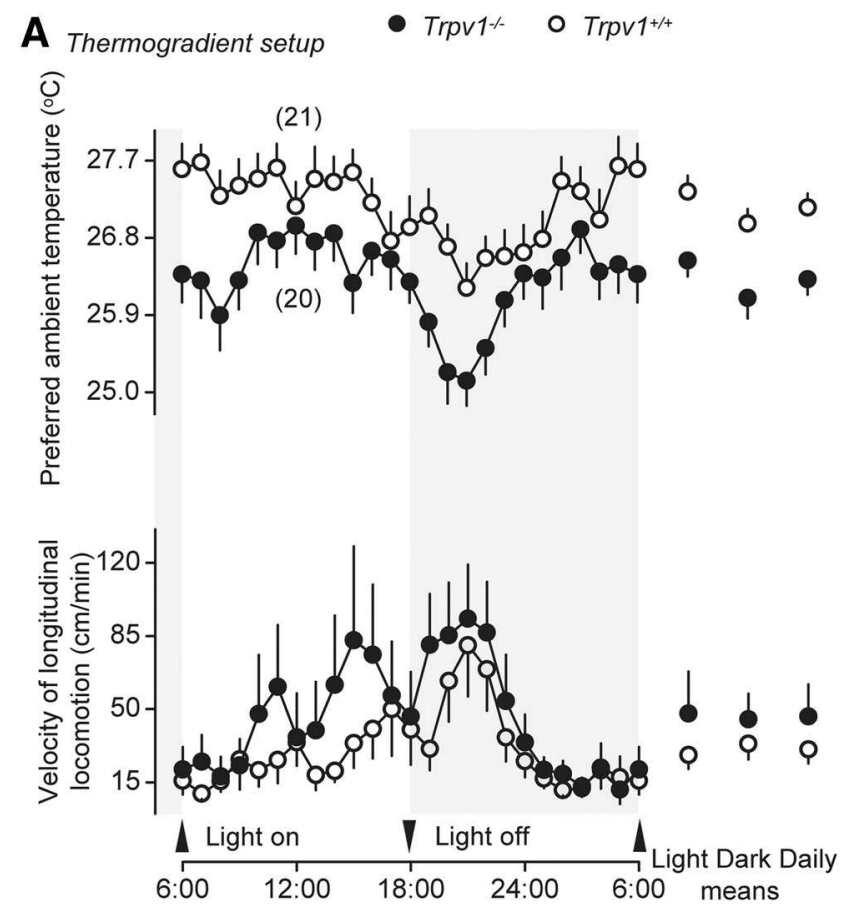

B Respirometry setup

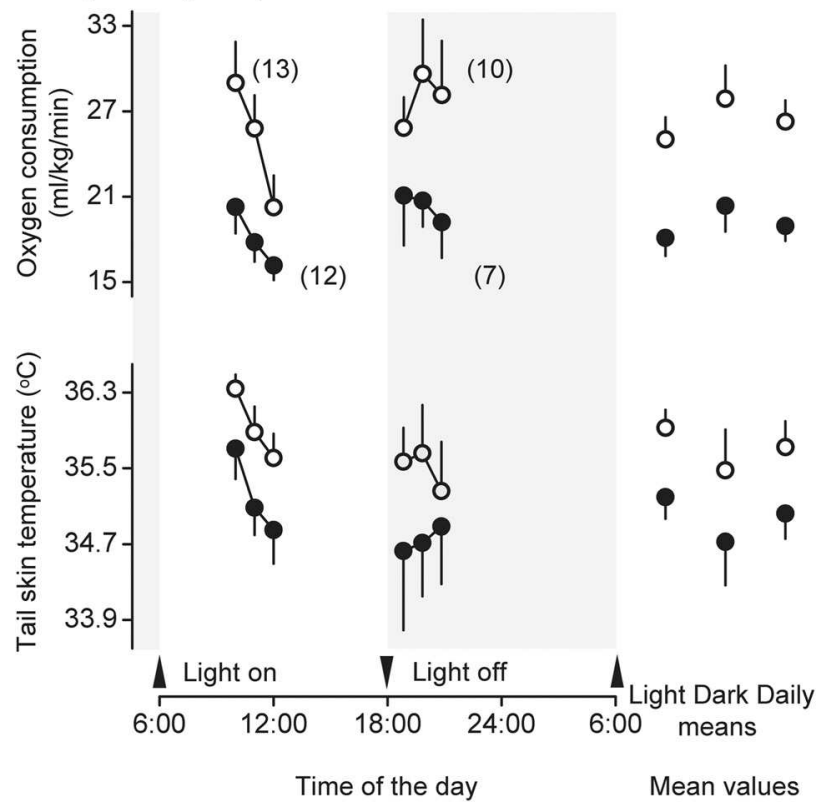

Figure 3. Compared to genetically unaltered controls, $\operatorname{Trpv} 1 \mathrm{~K} 0$ mice regulate $T_{\mathrm{b}}$ by using a different thermoeffector pattern. In the thermogradient setup $(\boldsymbol{A})$, they prefer a lower $T_{\mathrm{a}}$ and are hyperactive. In the respirometry setup $(\boldsymbol{B})$, they are hypometabolic (have a lower $V_{0_{2}}$ ) and vasoconstricted (have a lower tail $T_{\text {sk }}$ ).

counterparts (daily means of $26.3 \pm 0.2$ vs $27.2 \pm 0.2^{\circ} \mathrm{C}$, respectively; $p<0.01$ ), regardless of the phase. Trpv1 KO mice also expressed a higher locomotor activity, especially during the light phase. Although the mean velocities during the light phase, the dark phase, or the entire day did not differ statistically between the genotypes (Student's $t$ test), the $24 \mathrm{~h}$ activity curves were significantly different (ANOVA, $p<0.01$ ). From the biological point of view, the magnitude of the effect was remarkable: at one time point during the inactive phase (3:00 P.M.), Trpv1 KO mice were moving in the thermogradient with an average linear veloc- 
ity of $85 \mathrm{~cm} / \mathrm{min}(>1.2 \mathrm{~km} / \mathrm{d})$, which was $\sim 3$ times higher than the average speed of wild-type mice at the same time of day. Furthermore, it was higher than the locomotion speed of wildtype mice at any time, including the peak of their activity at night. When we measured autonomic thermoeffector activity in the respirometry setup, we found that $\operatorname{Trp} v 1 \mathrm{KO}$ mice maintained a lower metabolic rate and exhibited a more pronounced cutaneous vasoconstriction than the controls, regardless of the phase. The means for $V_{\mathrm{O}_{2}}$ and $T_{\text {sk }}$ for the $\mathrm{KO}$ versus control mice were $18.9 \pm 1.0$ versus $26.3 \pm 1.3 \mathrm{ml} / \mathrm{kg} / \mathrm{min}(p<0.0001)$ and $35.0 \pm$ 0.2 versus $35.7 \pm 0.2^{\circ} \mathrm{C}(p<0.05)$, respectively. The difference in $V_{\mathrm{O}_{2}}$ recorded was not contaminated by the tendency of the two genotypes to have different levels of locomotor activity, as these experiments were performed in restrained animals. In summary, under basal conditions, Trp $1 \mathrm{KO}$ mice have a thermoregulatory phenotype that reveals itself in a distinct thermoeffector pattern. These mice are prone to select a cooler environment; they are also hyperactive, hypometabolic, and vasoconstricted.

\section{Trpv1 KO mice have a higher TNZ}

The TNZ can be defined as a $T_{\mathrm{a}}$ range in which regulation of deep $T_{\mathrm{b}}$ is achieved primarily by changing the vasomotor tone in specialized heat-exchange organs, such as the mouse or rat tail (Romanovsky et al., 2002). Below the TNZ, rodent tails exhibit continuous skin vasoconstriction. Above the TNZ, they show continuous skin vasodilation. Within the TNZ, the tone of tail skin vasculature changes between moderate constriction and moderate dilation and exhibits a high intrasubject and intersubject variation (Romanovsky et al., 2002; Rudaya et al., 2005; Almeida et al., 2006). The fact that Trpv1 KO mice exhibited stronger tail skin vasoconstriction at $T_{\mathrm{a}}$ of $33.0^{\circ} \mathrm{C}$ in the respirometry setup (Fig. 3B), warranted further investigation. Hence, we assessed the TNZ of Trpv1 $1^{-/-}$and $\operatorname{Tr} p v 1^{+/+}$C57BL/6x129 mice in the thermocouple setup by measuring radiant tail $T_{\mathrm{sk}}$ by infrared thermography. At a high $T_{\mathrm{a}}$ of $35.0^{\circ} \mathrm{C}$ (Fig. $4 A$ ), the tails of all mice studied were strongly and uniformly vasodilated. Under these conditions, $T_{\text {sk }}$ was approaching deep $T_{\mathrm{b}}$ and was substantially higher than $T_{\mathrm{a}}$, which made the tails readily visible on the thermograms. In contrast, the tails of all mice were strongly vasoconstricted at a low $T_{\mathrm{a}}$ of $30.0^{\circ} \mathrm{C}$ (Fig. $4 \mathrm{H}$ ). Under these conditions, $T_{\mathrm{sk}}$ was approaching $T_{\mathrm{a}}$, which made the tails invisible on the thermograms. At all $T_{\mathrm{a}} \mathrm{s}$ between 30.0 and $35.0^{\circ} \mathrm{C}$, the extent of tail skin vasodilation/vasoconstriction varied between the animals, and $\operatorname{Trp} v 1^{-/-}$mice always showed stronger vasoconstriction than their $\operatorname{Trp} v 1^{+/+}$counterparts. At some $T_{\mathrm{a}} \mathrm{s}$ (e.g., $\left.32.0^{\circ} \mathrm{C}\right)($ Fig. $4 E)$, the tails of all $\operatorname{Trp} v 1 \mathrm{KO}$ mice were invisible $\left(T_{\mathrm{sk}}\right.$ close to $T_{\mathrm{a}}$ ), whereas the tails of all controls were readily visible $\left(T_{\text {sk }}>T_{\mathrm{a}}\right)$. In this case the intergenotype difference in $T_{\mathrm{sk}}$ exceeded $4^{\circ} \mathrm{C}$, which cannot be explained by a small difference in the deep $T_{\mathrm{b}}$ and indicates unequivocally a difference in the tail skin vasomotor tone. All control mice showed profound tail skin vasoconstriction at $T_{\mathrm{a}}<30.5^{\circ} \mathrm{C}$ and profound vasodilation at $T_{\mathrm{a}}>32.0^{\circ} \mathrm{C}$, thus suggesting that their TNZ in the setup studied was somewhere between 30.5 and $32.0^{\circ} \mathrm{C}$. In the same setup, all Trpv1 $\mathrm{KO}$ mice showed profound tail skin vasoconstriction at $T_{\mathrm{a}}<31.5^{\circ} \mathrm{C}$ and profound vasodilation at $T_{\mathrm{a}}>33.5^{\circ} \mathrm{C}$, thus suggesting that their TNZ in this setup was somewhere between 31.5 and $33.5^{\circ} \mathrm{C}$. To assess the extent of vasodilation quantitatively, we determined the optical density value for each tail image shown in Figure 4. An analysis of these values (two-way ANOVA) showed that the effect of the genotype was highly significant ( $p=$ 0.0001). Hence, Trpv1 KO mice have a higher TNZ than wildtype mice.
Positions 1, 3, and 5: $\operatorname{Trpv1} 1^{-1}$

Positions 2, 4, and 6: $\bigcirc \operatorname{Trpv1} 1^{+/+}$
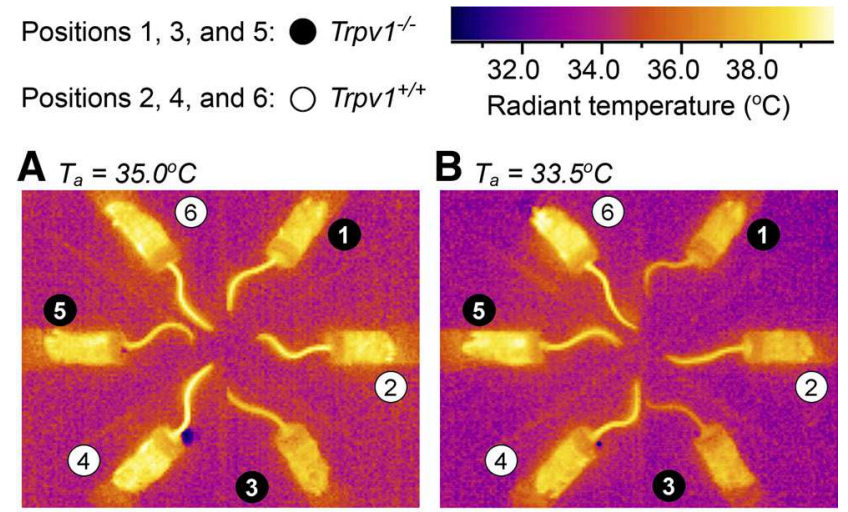

C $T_{a}=33.0^{\circ} \mathrm{C}$

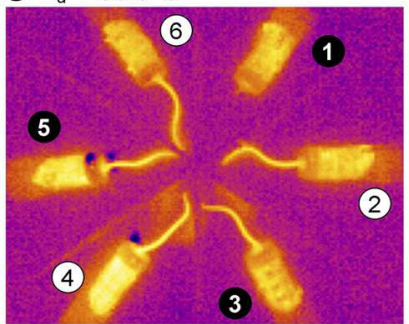

D $T_{a}=32.5^{\circ} \mathrm{C}$
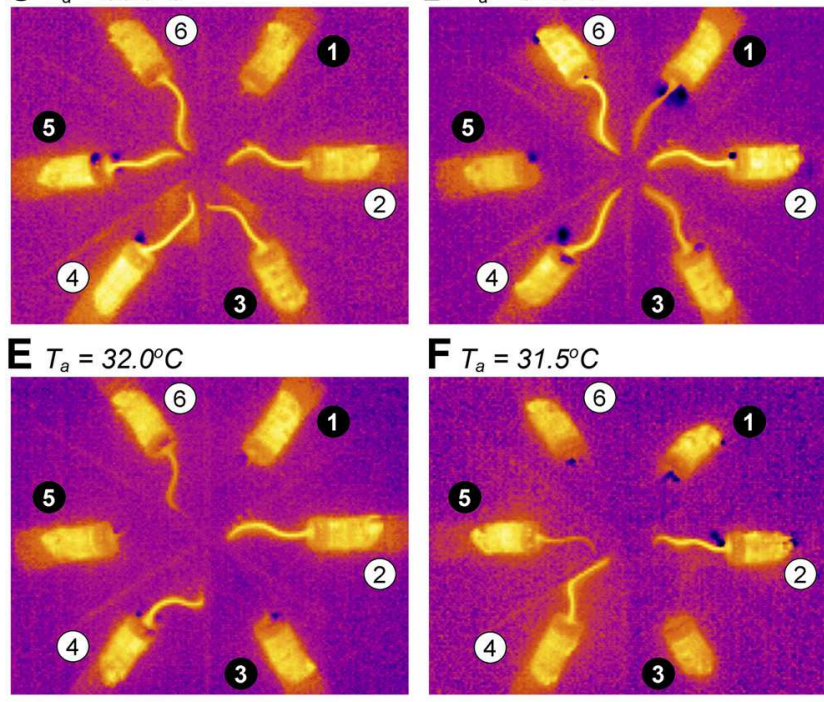

G $T_{a}=30.5^{\circ} \mathrm{C}$

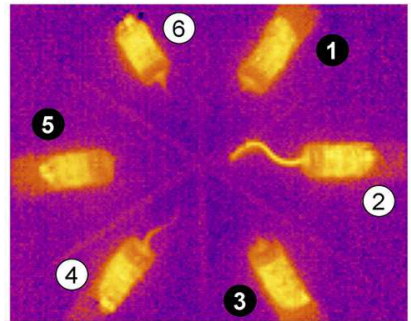

$\mathbf{H}_{T_{a}}=30.0^{\circ} \mathrm{C}$

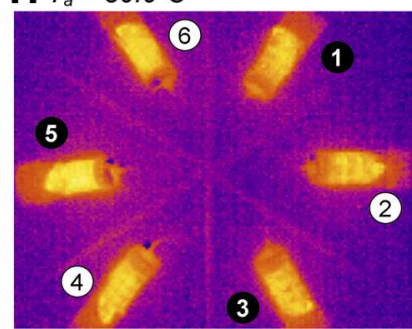

Figure 4. Trpv1 $\mathrm{K} 0$ mice have a higher TNZ. Three $\operatorname{Trpv} 1 \mathrm{KO}$ and three control mice in their confiners were situated in the thermocouple setup as slices of a pizza pie, so that their tails pointed to the center of the "pizza." In this setup, infrared thermograms were taken at different $T_{\mathrm{a}} \mathrm{s}: 35.0(\boldsymbol{A}), 33.5(\boldsymbol{B}), 33.0(\boldsymbol{C}), 32.5(\boldsymbol{D}), 32.0(\boldsymbol{E}), 31.5(\boldsymbol{F}), 30.5(\boldsymbol{G})$, and $30.0^{\circ} \mathrm{C}(\boldsymbol{H})$. It is known (see Results) that tails of rats and mice exhibit continuous skin vasodilation above the TNZ and continuous skin vasoconstriction below the TNZ. Note that all tails are clearly visible (vasodilated) at the highest $T_{a}(\boldsymbol{A})$ and completely invisible (vasoconstricted) at the lowest $T_{\mathrm{a}}(\boldsymbol{H})$. As $T_{\mathrm{a}}$ decreases (from $\boldsymbol{B}$ to $\boldsymbol{G}$ ), Trpv1 K0 mice become vasoconstricted faster than wild-type mice. Because within the TNZ, the tone of tail skin vasculature constantly changes between moderate constriction and moderate dilation and exhibits a high intrasubject and intersubject variation (Romanovsky et al., 2002), we conclude that the TNZ in this setup is $31.5-33.5^{\circ} \mathrm{C}$ for $\operatorname{Trpv} 1 \mathrm{KO}$ mice and $30.5-32.0^{\circ} \mathrm{C}$ for the controls.

\section{The thermoregulatory response of $\operatorname{Trpv} 1 \mathrm{KO}$ mice to heat} is unaltered

After we found that tail skin vasomotor tone was altered in $\operatorname{Tr} p v 1$ KO mice (Figs. 3B, 4), we studied the thermoregulatory response of these mice to ambient heating. To reveal even a small deficiency in heat defenses, we used a severe heat exposure model that results in a $\sim 3^{\circ} \mathrm{C}$ rise in deep $T_{\mathrm{b}}$. When exposed to heat, the mice 
of both genotypes (Trpv1 $1^{-/-}$and $\left.\operatorname{Tr} p v 1^{+/+}\right)$responded with rapid, near-maximal tail skin vasodilation with $T_{\text {sk }}$ approaching $40^{\circ} \mathrm{C}$ (Fig. 5A). Neither the $T_{\text {sk }}$ response nor the $T_{\mathrm{c}}$ response differed between the genotypes. Hence, despite the strong attenuation of heat-induced tail skin vasodilation reported in rats desensitized pharmacologically with TRPV1 agonists (for reviews, see Hori, 1984; Romanovsky et al., 2009), Trpv1 KO mice are fully capable of increasing heat loss through their tails and defending their deep $T_{\mathrm{b}}$ against heat. These results confirm the earlier observations by Szelényi et al. (2004) and Iida et al. (2005) showing that autonomic heat defenses of Trpv1 KO mice are not compromised.

\section{Trpv1 KO mice can maintain cold-induced thermogenesis} longer than genetically unaltered controls

To reveal even a small deficiency in cold defenses, we used a severe cold exposure model that results in a pronounced drop in deep $T_{\mathrm{b}}$. When exposed to cold in this model, the mice of both genotypes responded with tail skin vasoconstriction (a decrease in $T_{\text {sk }}$ ) and cold-induced thermogenesis (an increase in $V_{\mathrm{O}_{2}}$ ), but despite these cold-defense responses, their $T_{c}$ decreased by $>14^{\circ} \mathrm{C}$ (Fig. $5 \mathrm{~B}$ ). The response dynamics differed between the two genotypes. Whereas $V_{\mathrm{O}_{2}}$ of $\operatorname{Tr} p v 1 \mathrm{KO}$ mice reached a plateau at $\sim 60$ min after the beginning of cold exposure and was then maintained at this level until the end of the experiment (150 min), $V_{\mathrm{O}_{2}}$ of the controls reached the same level, but then started decreasing at $\sim 100 \mathrm{~min}(p<0.0001)$ and caused a corresponding decrease in $T_{\mathrm{c}}(p<0.0001)$. These data show that even though Trpv1 KO mice have a lower metabolic rate under basal conditions, they can maintain cold-induced thermogenesis for a somewhat longer period of time than genetically unaltered mice. An earlier study by Iida et al. (2005) did not find a difference in the thermoregulatory response of $\operatorname{Tr} p v 1 \mathrm{KO}$ mice to cold, but the authors used a much milder cold exposure. The combination of physical factors affecting the heat exchange between an animal and its environment ( $T_{\mathrm{a}}$, air humidity, air velocity, etc.) together with the use of confinement in our experimental paradigm resulted in a much greater decrease in deep $T_{\mathrm{b}}$ of the wild-type mice than that observed in the study by Iida et al. (2005): $14.2 \pm 1.1$ versus $1.2 \pm 0.5^{\circ} \mathrm{C}$. On the other hand, our results agree with those of Motter and Ahern (2008), who used stronger cooling (a $2.0-3.4^{\circ} \mathrm{C}$ decrease in $T_{\mathrm{b}}$ of wild-type mice) than Iida et al. (2005) and found that $\operatorname{Trp} v 1 \mathrm{KO}$ mice could defend their deep $T_{\mathrm{b}}$ against environmental cold somewhat better than their wild-type counterparts. Whether the slightly enhanced thermogenic capacity of the Trpv1 KO mice observed in this experiment was due to nonshivering or shivering thermogenesis, or both, is unknown.

\section{Peripheral administration of an exogenous TRPV1 agonist decreases stress-induced locomotion via an action on the TRPV1 channel}

After finding that Trpv1 KO mice were hyperactive (Fig. 3), we tested whether RTX, an ultrapotent exogenous TRPV1 agonist, decreases the gross locomotor activity. For this, we administered RTX intraperitoneally to $\operatorname{Tr} p v 1^{-/-}$mice with a C57BL/6x129 genetic background and to two strains of $\operatorname{Tr} p v 1^{+/+}$mice, C57BL/ 6x129 and C57BL/6. These experiments (and all experiments to follow) were performed in the telemetry setup. Because the expected effect of RTX was a decrease in the gross locomotor activity, RTX or its vehicle was administered acutely by briefly restricting the animal and pricking it with a needle (see Materials and Methods, Drugs and drug administration). In this paradigm, the handling and needle pricking associated with drug administration elevate the low activity typical for habituated mice during
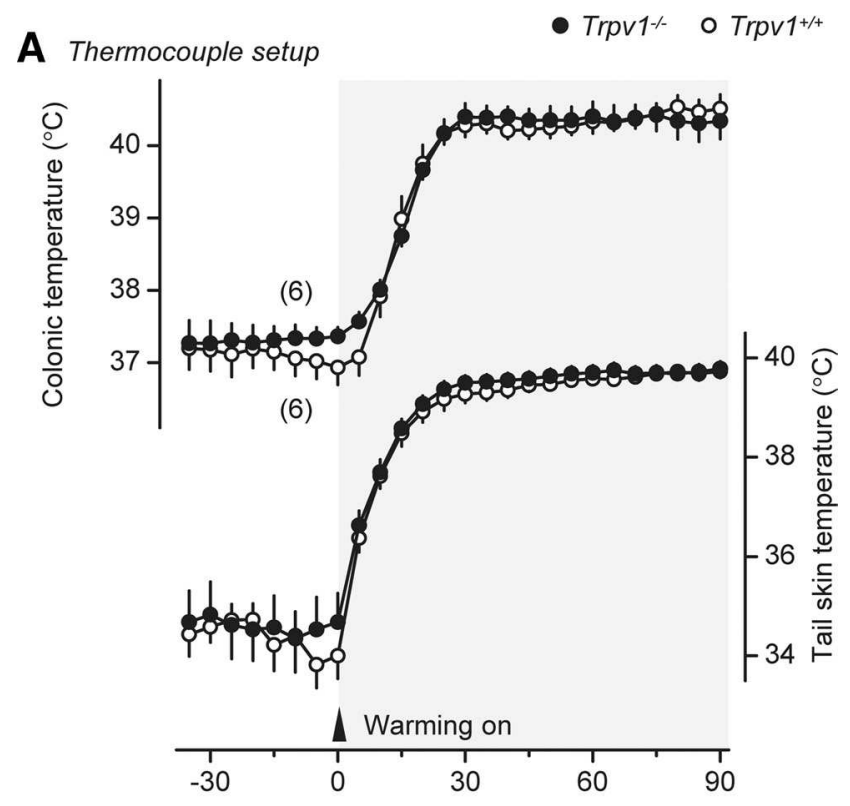

B Respirometry setup

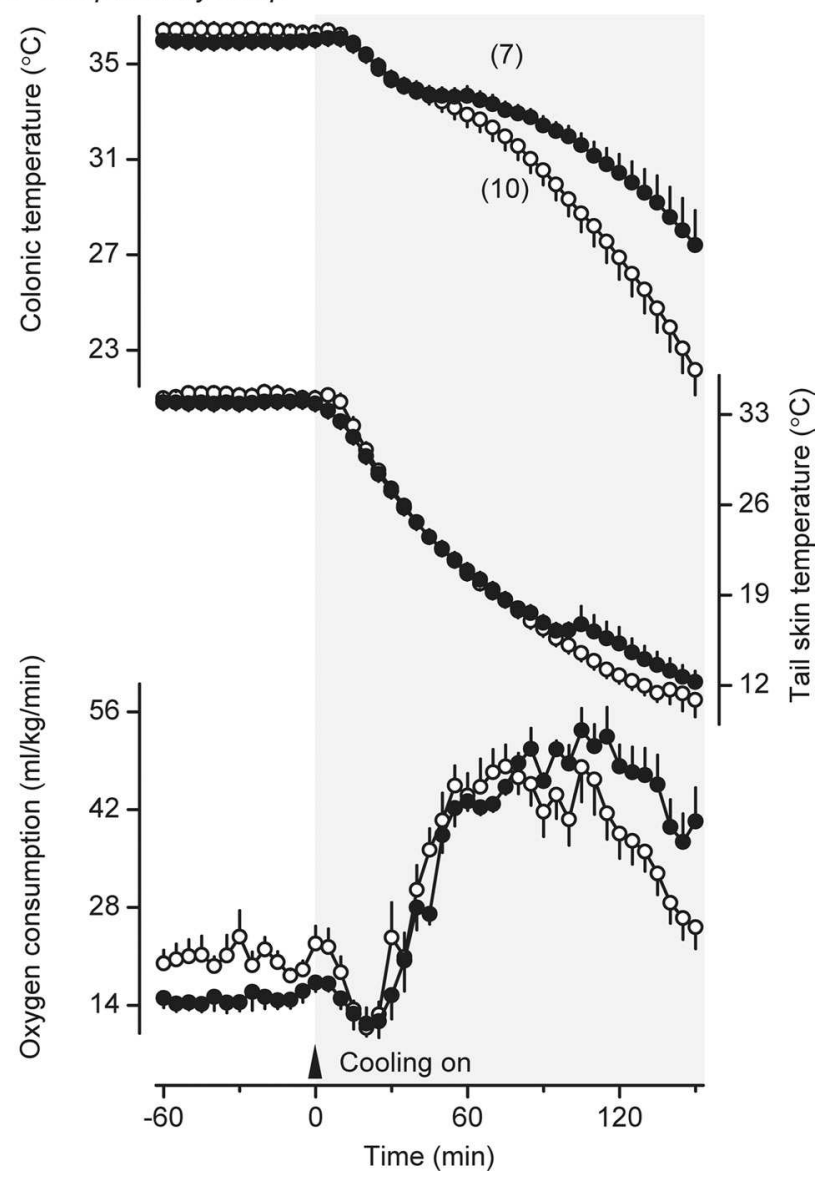

Figure 5. Thermoeffector and deep $T_{\mathrm{b}}$ responses of $\operatorname{Trpv} 1 \mathrm{~K} 0$ and genetically unaltered confined mice to severe heat exposure $(\boldsymbol{A})$ and severe cold exposure $(\boldsymbol{B})$. During heat exposure, $T_{a}$ was first raised from $31.0^{\circ} \mathrm{C}$ to $39.0^{\circ} \mathrm{C}$ at a rate of $\sim 0.3^{\circ} \mathrm{C} /$ min and then maintained at $39.0^{\circ} \mathrm{C}$ until the end of the experiment. Deep $T_{\mathrm{b}}$ (colonic) and tail skin vasodilation $\left(T_{\mathrm{sk}}\right)$ responses of the two genotypes to heat exposure were identical. During cold exposure, $T_{\mathrm{a}}$ was decreased from $33.0^{\circ} \mathrm{C}$ to $5.0^{\circ} \mathrm{C}$ at a mean rate of $0.2^{\circ} \mathrm{C} / \mathrm{min}$. In response to cold exposure, Trpv1 $\mathrm{K} 0$ mice maintain an increased thermogenesis $\left(V_{\mathrm{O}_{2}}\right)$ longer, thus better defending their $T_{\mathrm{b}}$ against severe cold. 
the light phase. The well known ability of RTX to decrease $T_{\mathrm{b}}$ (for review, see Romanovsky et al., 2009) was also evaluated, and to allow the animals to readily decrease their $T_{\mathrm{b}}$, the experiments were performed at a slightly subneutral $T_{\mathrm{a}}$ of $26.0^{\circ} \mathrm{C}$. The administration of the vehicle caused moderate hyperactivity and typical stress hyperthermia in all genotypes tested (Fig. 6). RTX (200 ng/kg, i.p.) blocked both the hyperactivity and hyperthermia responses in $\operatorname{Trp} v 1^{+/+} \mathrm{C} 57 \mathrm{BL} / 6$ and C57BL/6x129 mice $(p<0.0001$ for both responses in both strains) (Fig. 6A,B), even though the low dose of RTX used did not cause hypothermia. These results agree with studies showing that capsaicin, another exogenous TRPV1 agonist, decreases locomotor activity in rats and mice (Di Marzo et al., 2000, 2001; Proulx et al., 2005; Lee et al., 2006), and that both RTX (at higher doses) and capsaicin cause hypothermia in many mammalian species [for review, see Romanovsky et al. (2009)]. In the present study, the same dose of RTX that caused the anti-hyperkinetic and anti-hyperthermic effects in the control mice did not affect the stress-induced changes in $T_{\mathrm{ab}}$ or locomotor activity in Trpv1 KO mice (Fig. 6C). These results indicate that peripherally administered RTX decreases stress-induced locomotor activity and hyperthermia in mice by acting on TRPV1 channels. It was important to demonstrate an involvement of TRPV1 channels, because some effects of exogenous TRPV1 agonists (Lundbaek et al., 2005), including thermoregulatory effects (Dogan et al., 2004; Nikami et al., 2008), are not TRPV1 mediated.

\section{Peripheral administration of an endogenous TRPV1 agonist decreases stress-induced locomotion via an action on the TRPV1 channel}

We then tested whether AEA, an endocannabinoid with a full agonistic activity against TRPV1, affects $T_{\mathrm{ab}}$ and stress-induced locomotion in a way similar to RTX. In this experiment, the acute intraperitoneal injection of the vehicle resulted in an increase in the locomotor activity, similar to those shown in Figure 6, but did not cause stress hyperthermia. The blunted effect of stress on $T_{\mathrm{ab}}$ was probably due to the fact that a large volume of a roomtemperature vehicle was used to deliver the high dose of AEA: 30 $\mathrm{ml} / \mathrm{kg}$ ( $\sim 0.9 \mathrm{ml}$ per mouse) in this experiment (Fig. 7) as compared to $3.3 \mathrm{ml} / \mathrm{kg}$ ( $\sim 0.1 \mathrm{ml}$ per mouse) in the RTX experiment (Fig. 6). As compared to its vehicle, AEA (15 mg/kg, i.p.) significantly decreased $T_{\mathrm{ab}}$ and strongly attenuated locomotor activity (suppressed stress hyperkinesis) in $\operatorname{Tr} p v 1^{+/+}$mice of both strains: C57BL/6 $\left(p<0.0001\right.$ for $T_{\mathrm{ab}} ; p<0.05$ for locomotor activity) (Fig. 7A) and C57BL/6x129 ( $p<0.05$ for $T_{\mathrm{ab}} ; p<0.01$ for locomotor activity) (Fig. $7 B$ ). These results agree with the reported hypothermic and hypokinetic effects of AEA, administered to rats and mice at high doses (Crawley et al., 1993; Fride and Mechoulam, 1993; Smith et al., 1994; Adams et al., 1998; Watanabe et al., 1999; Wiley et al., 2006; Wise et al., 2007). How-
B $\operatorname{Trpv}^{+/+} \mathrm{C} 57 \mathrm{BL} / 6 \times 129$ C Trpv1\% C57BL/6x129

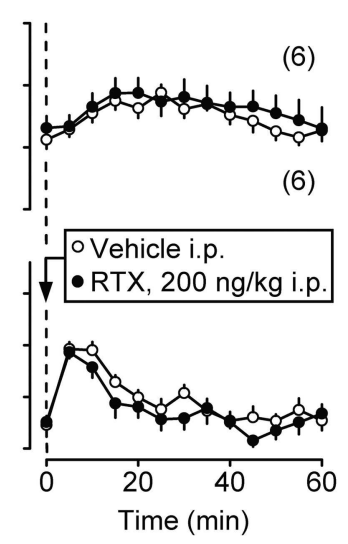

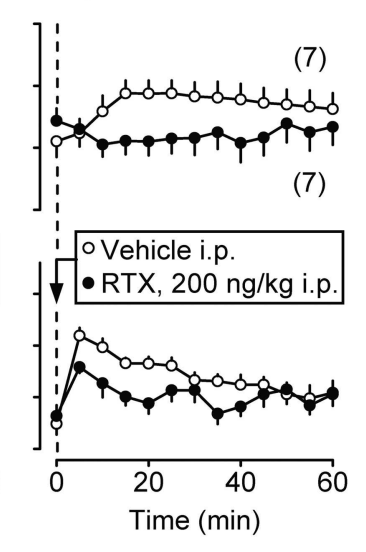

(6)
$(6)$

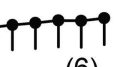

(6)

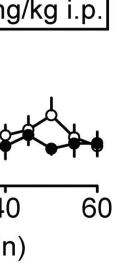

Figure 6. Intraperitoneal RTX (200 ng/kg) attenuates the injection-induced hyperthermia and hyperkinesis in $\operatorname{Trpv} 1^{+/+}$mice of two strains, C57BL/6 (A) and C57BL/6x129 (B), but not in Trpv1 ${ }^{-/-}$C57BL/6x129 mice (C). These experiments and those

A Trpv1+/+ C57BL/6

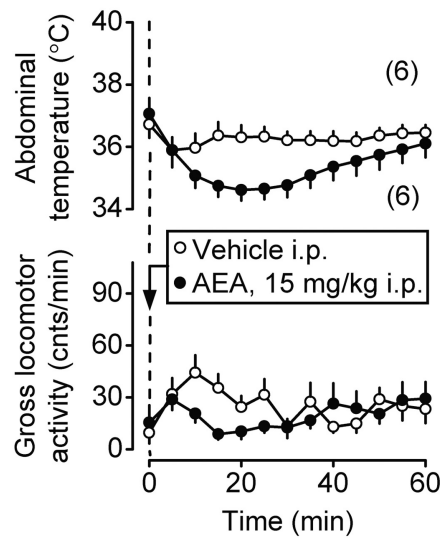

B Trpv1+/+ C57BL/6x129

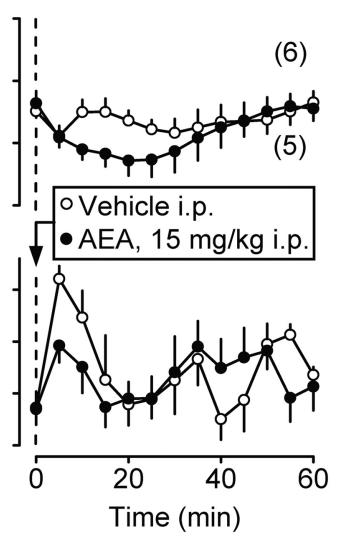

Trpv1 $\% 57 \mathrm{BL} / 6 \times 129$

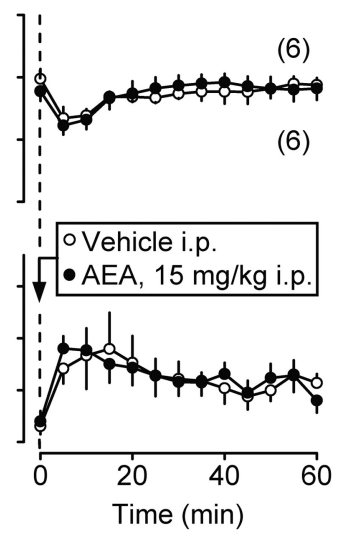

Figure 7. Intraperitoneal AEA ( $15 \mathrm{mg} / \mathrm{kg})$ causes hypothermia and attenuates the injection-induced hyperkinesis in $\operatorname{Trpv} 1^{+/+}$ mice of two strains, C57BL/6 (A) and C57BL/6x129 (B), but not in Trpv1 ${ }^{-1-}$ C57BL/6x129 mice (C).

ever, none of these effects occurred when AEA was injected in Trpv1 $1^{-1-}$ C57BL/6x129 mice (Fig. 7C). Even though AEA also acts as a full agonist at the cannabinoid-1 receptor (Vogel et al., 1993) and as a partial agonist at the cannabinoid-2 receptor (Gonsiorek et al., 2000), the complete absence of both $T_{\mathrm{b}}$ decreasing and activity-decreasing effects of AEA in Trpv1 KO mice shows that cannabinoid receptors do not mediate the effects studied. This is the first observation showing directly that AEA suppresses deep $T_{\mathrm{b}}$ and locomotor activity by acting on the TRPV1 channel.

Peripheral administration of a selective TRPV1 antagonist produces hyperkinesis via an action on the TRPV1 channel Next, we studied the effect of AMG0347, a TRPV1 antagonist, on deep $T_{\mathrm{b}}$ and locomotor activity of $\operatorname{Trp} v 1^{-/-}$and $\operatorname{Trp} v 1^{+/+}$ C57BL/6x129 mice. Based on the results with TRPV1 agonists (Figs. 6, 7), we expected the antagonist to cause an increase in the locomotor activity. To study the expected increase, we avoided inducing stress hyperkinesis and infused AMG0347 or its vehicle in a nonstressful manner, through a preimplanted intraperitoneal catheter, from outside of the climatic chamber. Because the expected thermal effect was hyperthermia (Steiner et al., 2007; Garami et al., 2010), these experiments were conducted at $T_{\mathrm{a}}$ of 
A $\operatorname{Trpv}^{1+/+} \mathrm{C} 57 \mathrm{BL} / 6 \times 129$

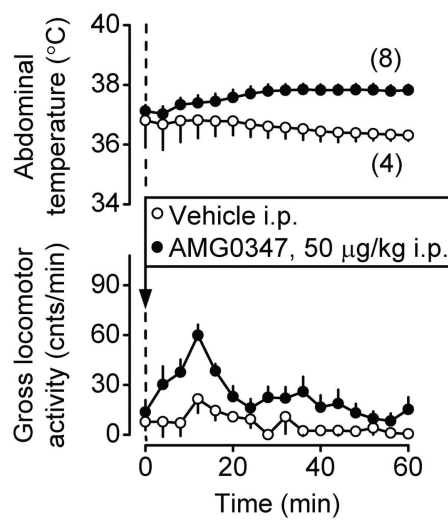

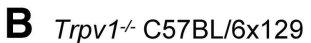

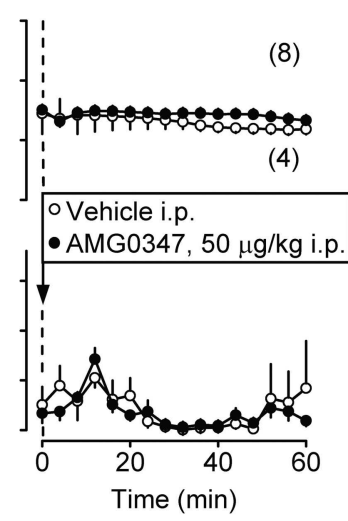

Figure 8. Intraperitoneal AMG0347 (50 $\mu \mathrm{g} / \mathrm{kg}$ ) causes hyperthermia and locomotor hyperactivity in wild-type mice $(\boldsymbol{A})$ but not in $\operatorname{Trpv} 1 \mathrm{KO}$ mice $(\boldsymbol{B})$. These experiments were performed in the telemetry setup at a neutral $T_{\mathrm{a}}$ of $31.0^{\circ} \mathrm{C}$.

$31.0^{\circ} \mathrm{C}$, which is neutral for both $\operatorname{Trpv} 1 \mathrm{KO}$ and control mice in the telemetry setup (Table 1). As compared to its vehicle, AMG0347 (50 $\mu \mathrm{g} / \mathrm{kg}$, i.p.) caused hyperthermia and hyperkinesis in Trpv1 $1^{+/+}$mice $(p<0.0001$ for both) (Fig. $8 A)$. Whereas it is well known that TRPV1 antagonists cause hyperthermia (for reviews, see Gavva, 2008; Romanovsky et al., 2009), the observed increase in the locomotor activity was an unexpected finding, because earlier studies in rats with several TRPV1 antagonists reported no changes in the locomotor activity (Gavva et al., 2005; Cui et al., 2006; Drizin et al., 2006; Mills et al., 2008). However, the TRPV1 antagonists in all these studies were administered in a stressful way (often as intragastric gavage), and the resultant stress-induced hyperkinesis could have masked the short-lived and modest increase in the locomotor activity seen in the present study. The same dose of AMG0347 that caused hyperthermia and hyperkinesis in the control mice affected neither $T_{\mathrm{ab}}$ nor gross locomotor activity in Trpv1 KO mice (Fig. $8 B$ ). Whereas it has been shown in our previous studies that TRPV1 antagonists cause hyperthermia by acting on TRPV1 channels (Steiner et al., 2007; Garami et al., 2010), the present study is the first to demonstrate a hyperkinetic effect for AMG0347 and to show that this effect occurs due to an action at the TRPV1 channel.

\section{A TRPV1 agonist suppresses stress-induced locomotion by acting outside the brain}

We then attempted to determine whether the anti-hyperkinetic response to intraperitoneal RTX (Fig. 6) occurs due to an action inside or outside the brain. A very low dose of RTX, $20 \mathrm{ng} / \mathrm{kg}$, was used in this experiment and administered either intraperitoneally or into the lateral brain ventricle. When administered intraperitoneally, this dose attenuated the injection-induced hyperthermia and the locomotor response ( $p<0.0001$ for both) (Fig. 9A). However, the same dose of RTX administered intracerebroventricularly affected neither parameter (Fig. 9B). These data suggest that RTX affects locomotion by acting outside the blood-brain barrier. It should be noted that the low doses of RTX used in the present study, $20 \mathrm{ng} / \mathrm{kg}$ (Fig. 9) and $200 \mathrm{ng} / \mathrm{kg}$ (Fig. 6), did not cause a typical hypothermic response, but did decrease the injection-associated rise in $T_{\mathrm{b}}$. Such an action on $T_{\mathrm{b}}$ is likely to be secondary to the decrease in locomotor activity. At higher doses, RTX readily causes marked hypothermia (de Vries and Blumberg, 1989; Romanovsky et al., 2009), and the marked hypother-

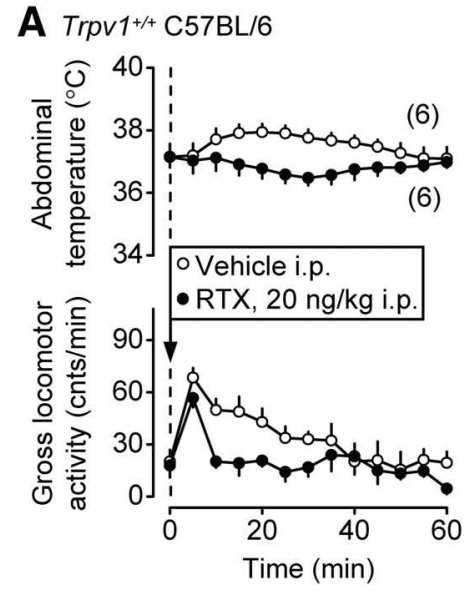

B $\operatorname{Trpv1+/}$ C57BL/6

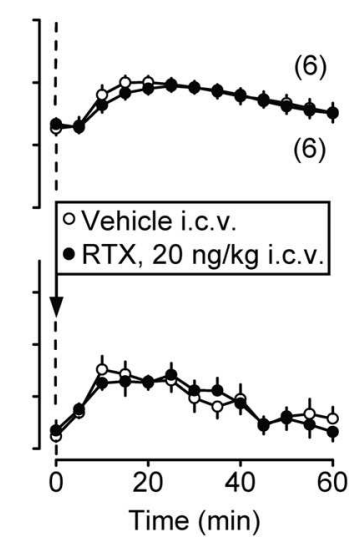

Figure 9. The intraperitoneal $(\boldsymbol{A})$, but not intracerebroventricular $(\boldsymbol{B})$, administration of RTX at a dose of $20 \mathrm{ng} / \mathrm{kg}$ attenuates the injection-induced hyperthermia and hyperkinesis in Trpv $1^{+/+}$C57BL/6 mice.

mic response to RTX is thought to be due to a central, rather than peripheral, action [for review, see Romanovsky et al. (2009)].

\section{Aged Trpv1 KO mice are heavier than genetically} unaltered controls

As a side finding, we noticed that, with age, Trpv1 KO mice of both sexes became heavier than their $\operatorname{Trp} v 1^{+/+}$counterparts (Fig. 10). For example, at the age of 8 months, the body mass of the male Trpv1 KO mice was $14 \%$ higher than that of the agematched male controls: $49 \pm 1$ versus $43 \pm 1 \mathrm{~g}(p<0.0001)$. The two heaviest mice in our colony were Trpv1 KO males, who had a body mass of $59 \mathrm{~g}$ (at the age of 8 months) and $58 \mathrm{~g}$ (at the age of 14 months). By the time we discovered that aged Trpv1 KO mice were significantly heavier than their wild-type counterparts, Amgen had terminated its colony, which made obtaining any additional measures (the amount and distribution of fat, blood lipids) impossible. Even though we did not measure the amount of fat, the aged Trpv1 KO mice looked obese, rather than proportionally large. They had larger bellies, a symptom consistent with accumulation of visceral fat, a generally accepted hallmark of aging (Huffman and Barzilai, 2009). We also noticed both a large amount of visceral fat and a thick layer of subcutaneous fat while performing surgeries on older Trpv1 KO mice for a different study (S. P. Wanner and A. A. Romanovsky, unpublished observations). The observed association of hyperactivity (recorded at a younger age) with overweight (recorded at an older age) seems paradoxical, but a similar relationship was reported for a group of healthy humans: those individuals who were more physically active when they were young showed a higher mass gain with age (Westerterp and Plasqui, 2009). Based on the present observation, it is tempting to speculate that TRPV1 channels protect from aging-associated obesity. From the thermoregulatory point of view, the increased body mass found in aged Trpv1 KO mice may have an insulating role, similar to that played by subcutaneous fat in animals living in cold climates (Blix and Steen, 1979).

\section{Discussion}

In agreement with previous studies (Szelényi et al., 2004; Iida et al., 2005), we have found no profound alterations in basal $T_{\mathrm{b}}$ in Trpv1 KO mice (Fig. 2). We have also confirmed that, compared to their $\operatorname{Trpv1}{ }^{+/+}$counterparts, $\operatorname{Trpv1} 1^{-/-}$mice have a slightly higher magnitude of circadian fluctuations in $T_{\mathrm{b}}$. The main thermoregulatory abnormality of $\operatorname{Tr} p v 1 \mathrm{KO}$ mice appears to be not an 

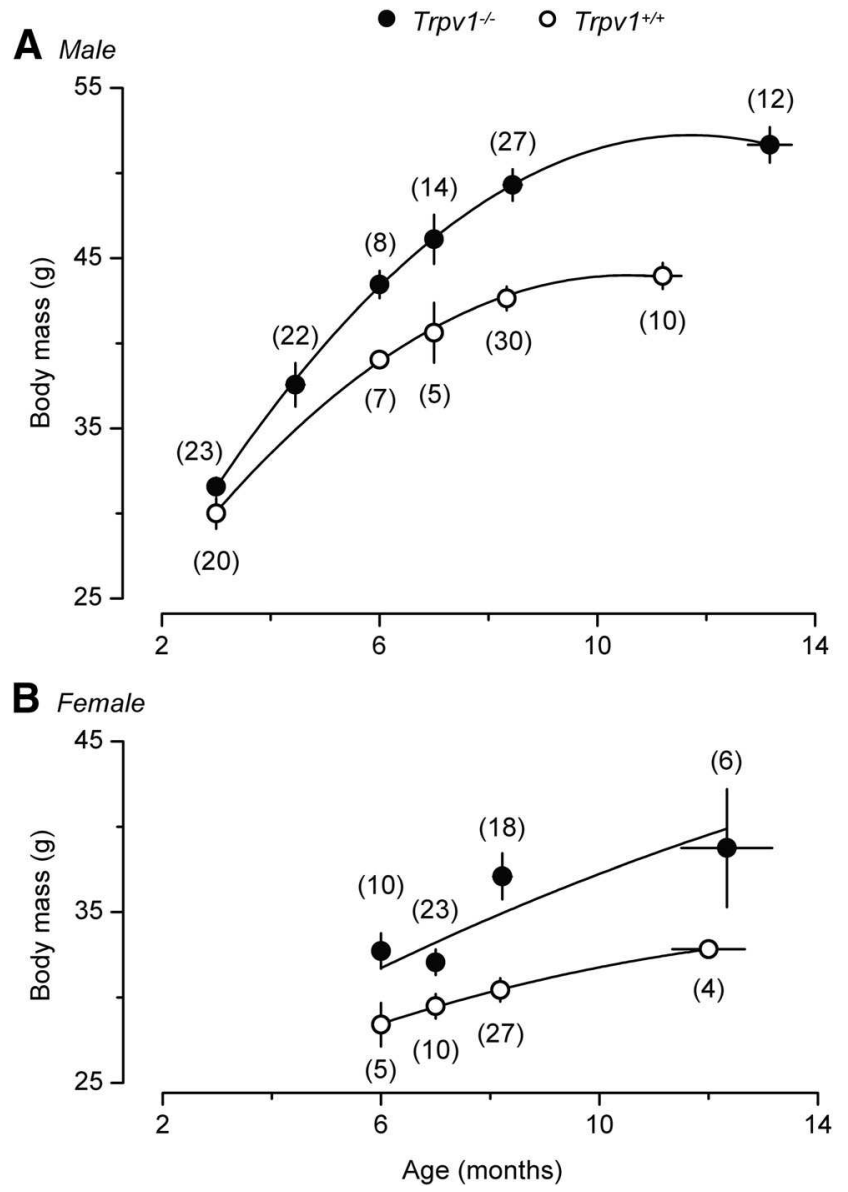

Figure 10. With age, both male $(\boldsymbol{A})$ and female $(\boldsymbol{B}) \operatorname{Trpv} 1 \mathrm{~K} 0$ mice become heavier than their age-matched controls.

altered level of $T_{\mathrm{b}}$, but a different pattern of thermoeffectors used to regulate $T_{\mathrm{b}}$ under various thermal, housing, and restraint conditions. Trpv1 KO mice were consistently hypometabolic (had a lower $V_{\mathrm{O}_{2}}$ ) and preferred a lower $T_{\mathrm{a}}$ than controls (Fig. 3). These thermoeffector changes tend to decrease $T_{\mathrm{b}}$. At the same time, the $\mathrm{KO}$ mice expressed two thermoeffector activities aimed at increasing $T_{\mathrm{b}}$. First, they had more pronounced tail skin vasoconstriction (Fig. $3 B$ ) and, in agreement with this, had a higher TNZ (Fig. 4). Second, they expressed strong hyperkinesis with the average locomotion velocity during the second half of the inactive phase exceeding that of wild-type mice during the active phase (Fig. $3 A$ ).

This finding of increased locomotor activity of $\operatorname{Tr} p v 1 \mathrm{KO}$ mice deserves a separate discussion, as it seems to contradict several studies showing that the deletion of the Trpv1 gene causes no changes in locomotion (Caterina et al., 2000; Davis et al., 2000; Marsch et al., 2007). However, all these studies used much shorter observation periods, sometimes as short as $15 \mathrm{~min}$. Furthermore, even though these studies found no statistically significant differences in the locomotor activity of Trpv1 KO mice, some of them showed strong tendencies that were in agreement with the present results. For example, Davis et al. (2000) found that Trpv1 KO mice tended to have higher scores in several activity tests. In the hole board test for exploratory behavior, the activity of Trpv1 KO mice exceeded that of wild-type mice by $92 \%$ $(p=0.12)$. An increased activity of $\operatorname{Tr} p v 1 \mathrm{KO}$ mice was also observed by Marsch et al. (2007) in several tests for anxietyrelated behaviors. In these tests, Trpv1 KO mice adapted to experimental conditions faster and expressed exploratory behav- iors associated with a higher locomotor activity. However, other studies have found no involvement of TRPV1 channels in anxiety behaviors (Bitencourt et al., 2008; Panlilio et al., 2009). The increased spontaneous locomotion observed in our study is also unlikely to be due to changes in anxiety or exploratory behavior, because the mice were extensively adapted to experimental conditions and observed over long periods of time. Whether the increased locomotion relates to changes in the sleep pattern is unknown, but TRPV1 antagonists do not seem to affect sleep (Takeda et al., 2008).

Because their autonomic thermoregulatory responses showed no attenuation in our study and in studies by others (Szelényi et al., 2004; Iida et al., 2005; Motter and Ahern, 2008), we directed our attention to the behavioral responses: the selection of $T_{\mathrm{a}}$, which is the classic example of thermoregulatory behavior, and the gross locomotor activity, which is viewed by many authors as a thermoregulatory effector in small rodents. In the thermogradient apparatus, we saw that $\operatorname{Tr} p v 1 \mathrm{KO}$ mice preferred a lower $T_{\mathrm{a}}$ and were hyperactive as compared to the controls (Fig. 3). Similar to how humans prefer to exercise in a cooler environment, experimental rodents select a lower $T_{\mathrm{a}}$ when their activity is higher. Such an adjustment of the preferred $T_{\mathrm{a}}$ accompanies the increase in the locomotor activity of rats (Gordon, 1994) and mice (Fig. 3) at the transition from the inactive (light) phase to the active (dark) phase. A similar adjustment accompanies the exploratory behavior of rats in a thermogradient apparatus when the animals are not adapted to the apparatus [for discussion, see Almeida et al. (2006)]. Having excluded profound deficiencies in the two major autonomic effectors directly (Fig. 5) and knowing that a decrease in the thermopreferendum often occurs secondarily to an increase in locomotor activity, we are tempted to speculate that the increased locomotion may be one of the "primary" symptoms of the Trpv1 KO phenotype. We then focused the second part of the study on locomotor activity.

We asked whether pharmacological manipulations with the activity of TRPV1 channels would affect the locomotor activity (and also $T_{\mathrm{b}}$ ). First, we showed that RTX (an exogenous TRPV1 agonist) and AEA (an endogenous TRPV1 agonist) both attenuated stress-induced hyperactivity in $\operatorname{Tr} p v 1^{+/+}$mice of two strains (Figs. 6, 7). This effect agrees well with the reported hypokinetic action of these and other TRPV1 agonists in several different tests (Di Marzo et al., 2001; Proulx et al., 2005; Wiley et al., 2006; Wise et al., 2007). Next, we studied the responses to RTX and AEA in Trpv1 KO mice. By showing that the locomotor activity response was unaffected by these TRPV1 agonists in Trpv1 KO mice, we have confirmed that the anti-hyperkinetic effect of either agonist occurs via an action on TRPV1. This is in agreement with the finding that the AEA-induced decrease in locomotor activity of rats is blocked by TRPV1 antagonists (de Lago et al., 2004; Lee et al., 2006; Tzavara et al., 2006). In the next experiment, we used AMG0347, a highly potent and selective TRPV1 antagonist. In addition to causing the well characterized hyperthermic effect (Steiner et al., 2007; Garami et al., 2010), a nonstressful intraperitoneal administration of AMG0347 also increased the locomotor activity in Trpv1 $1^{+/+}$mice (Fig. 8). However, AMG0347 did not increase locomotion in Trpv1 KO mice, thus indicating that the hyperkinetic effect of AMG0347 occurs via an action on TRPV1. Hence, our results with RTX, AEA, and AMG0347 support the hypothesis that TRPV1 channels tonically suppress the general locomotor activity.

We then asked whether the TRPV1 channels involved are located inside or outside the brain. To answer this question, we injected a very low dose of RTX (20 ng/ $\mathrm{kg}$ ) either intraperitoneally or into the lateral cerebral ventricle. The intraperitoneal ad- 
ministration was effective in decreasing the injection-associated locomotion, whereas the intracerebroventricular administration was not (Fig. 9), thus suggesting that RTX causes its antihyperkinetic activity by acting outside the blood-brain barrier. Such a peripheral action agrees with the predominant location of TRPV1 channels on polymodal sensory neurons in the dorsalroot and nodose ganglia (Szallasi et al., 1995; Caterina et al., 1997; Tominaga et al., 1998). These neurons are activated by a variety of stimuli and are thought to modulate a wide array of autonomic and behavioral functions (Caterina and Julius, 2001; Inoue et al., 2006; Nilius et al., 2007; Szallasi et al., 2007; Sharif-Naeini et al., 2008; Romanovsky et al., 2009). Tonic activation of peripheral TRPV1 channels has been shown to inhibit thermogenesis and heat retention in the study by Steiner et al. (2007), to modulate spinal locomotor networks in the study by Mandadi et al. (2009), and to inhibit the general locomotor activity in the present work.

As a side observation, we have found that, with age, Trpv1 KO mice of either sex (maintained on a regular diet) become heavier than their wild-type counterparts (Fig. 10). This observation seems to contradict studies by Davis et al. (2000), Rong et al. (2004), and Zhang et al. (2007), who found no changes in the body mass of Trpv1 KO mice compared to controls, when maintained on a regular or high-fat diet. It also contradicts the study by Motter and Ahern (2008), in which Trpv1 KO mice were found less prone than wild-type controls to become obese on a high-fat diet. However, all abovementioned studies used young mice with the body mass in a 10-30 g range (on a regular diet). In this body mass range, the intergenotype difference in our study was also small, but with age (we observed the mice up to the age of 14 months), the difference increased (Fig. 10). Interestingly, the obesity-protective role of TRPV1 channels has been proposed (Zhang et al., 2007; Suri and Szallasi, 2008) and is supported by two lines of evidence. First, Zhang et al. (2007) detected TRPV1 channels in preadipocytes, as well as in visceral adipose tissue from mice and humans. These authors demonstrated TRPV1 downregulation during regular adipogenesis and a reduced TRPV1 expression in visceral adipose tissue from obese mice and humans. Second, a long-term administration of capsaicin or capsinoids (nonpungent, capsaicin-related TRPV1 agonists) has been repeatedly shown to suppress visceral fat accumulation, induce thermogenesis, and prevent an increase in the body mass in laboratory animals and humans (Kawabata et al., 2006; Zhang et al., 2007; Snitker et al., 2009). Further studies of the role of the TRPV1 channel in obesity seem warranted.

In conclusion, the first part of the present study shows that Trpv1 KO mice possess a distinct thermoregulatory phenotype, which includes hypometabolism, enhanced skin vasoconstriction, preference for a lower $T_{\mathrm{a}}$, and an increased locomotor activity. The second part of our study shows that TRPV1 agonists and antagonists decrease and increase, respectively, the locomotor activity by acting on TRPV1 channels. For agonists, their effect on locomotion is due to an action outside the brain. We propose that TRPV1-mediated signals from the periphery tonically suppress general locomotor activity. Whether such suppression occurs due to a direct action on the locomotor system or indirectly (e.g., by affecting motivation or vigilance state) is a topic for future studies. The nature of the TRPV1-mediated signals that suppress locomotion also remains to be elucidated.

\section{Notes}

The curves of gross locomotor activity measured simultaneously by intraperitoneal telemetry probes and by external motion detectors in mice treated with different drugs are presented in supplemental
Figure 1 posted at http://www.feverlab.net/pages/publicationpdfs/ supplementJN2011.pdf. This material has not been peer reviewed.

\section{References}

Adams IB, Compton DR, Martin BR (1998) Assessment of anandamide interaction with the cannabinoid brain receptor: SR 141716A antagonism studies in mice and autoradiographic analysis of receptor binding in rat brain. J Pharmacol Exp Ther 284:1209-1217.

Almeida MC, Steiner AA, Branco LG, Romanovsky AA (2006) Cold-seeking behavior as a thermoregulatory strategy in systemic inflammation. Eur J Neurosci 23:3359-3367.

Berkley R, Jacobson E (1992) Peppers: a cookbook. New York: Simon and Schuster.

Bitencourt RM, Pamplona FA, Takahashi RN (2008) Facilitation of contextual fear memory extinction and anti-anxiogenic effects of AM404 and cannabidiol in conditioned rats. Eur Neuropsychopharmacol 18:849-859.

Blix AS, Steen JB (1979) Temperature regulation in newborn polar homeotherms. Physiol Rev 59:285-304.

Brown D, Livesey G, Dauncey MJ (1991) Influence of mild cold on the components of 24 hour thermogenesis in rats. J Physiol 441:137-154.

Caterina MJ, Julius D (2001) The vanilloid receptor: a molecular gateway to the pain pathway. Annu Rev Neurosci 24:487-517.

Caterina MJ, Schumacher MA, Tominaga M, Rosen TA, Levine JD, Julius D (1997) The capsaicin receptor: a heat-activated ion channel in the pain pathway. Nature 389:816-824.

Caterina MJ, Leffler A, Malmberg AB, Martin WJ, Trafton J, Petersen-Zeitz KR, Koltzenburg M, Basbaum AI, Julius D (2000) Impaired nociception and pain sensation in mice lacking the capsaicin receptor. Science 288:306-313

Christoph T, Bahrenberg G, De Vry J, Englberger W, Erdmann VA, Frech M, Kögel B, Röhl T, Schiene K, Schröder W, Seibler J, Kurreck J (2008) Investigation of TRPV1 loss-of-function phenotypes in transgenic shRNA expressing and knockout mice. Mol Cell Neurosci 37:579-589.

Crawley JN, Corwin RL, Robinson JK, Felder CC, Devane WA, Axelrod J (1993) Anandamide, an endogenous ligand of the cannabinoid receptor, induces hypomotility and hypothermia in vivo in rodents. Pharmacol Biochem Behav 46:967-972.

Cui M, Honore P, Zhong C, Gauvin D, Mikusa J, Hernandez G, Chandran P, Gomtsyan A, Brown B, Bayburt EK, Marsh K, Bianchi B, McDonald H, Niforatos W, Neelands TR, Moreland RB, Decker MW, Lee CH, Sullivan JP, Faltynek CR (2006) TRPV1 receptors in the CNS play a key role in broad-spectrum analgesia of TRPV1 antagonists. J Neurosci 26:9385-9393.

Davis JB, Gray J, Gunthorpe MJ, Hatcher JP, Davey PT, Overend P, Harries MH, Latcham J, Clapham C, Atkinson K, Hughes SA, Rance K, Grau E, Harper AJ, Pugh PL, Rogers DC, Bingham S, Randall A, Sheardown SA (2000) Vanilloid receptor-1 is essential for inflammatory thermal hyperalgesia. Nature 405:183-187.

de Lago E, de Miguel R, Lastres-Becker I, Ramos JA, Fernández-Ruiz J (2004) Involvement of vanilloid-like receptors in the effects of anandamide on motor behavior and nigrostriatal dopaminergic activity: in vivo and in vitro evidence. Brain Res 1007:152-159.

de Vries DJ, Blumberg PM (1989) Thermoregulatory effects of resiniferatoxin in the mouse: comparison with capsaicin. Life Sci 44:711-715.

Di Marzo V, Breivogel C, Bisogno T, Melck D, Patrick G, Tao Q, Szallasi A, Razdan RK, Martin BR (2000) Neurobehavioral activity in mice of N-vanillyl-arachidonyl-amide. Eur J Pharmacol 406:363-374.

Di Marzo V, Lastres-Becker I, Bisogno T, De Petrocellis L, Milone A, Davis JB, Fernandez-Ruiz JJ (2001) Hypolocomotor effects in rats of capsaicin and two long chain capsaicin homologues. Eur J Pharmacol 420:123-131.

Dogan MD, Patel S, Rudaya AY, Steiner AA, Székely M, Romanovsky AA (2004) Lipopolysaccharide fever is initiated via a capsaicin-sensitive mechanism independent of the subtype-1 vanilloid receptor. Br J Pharmacol 143:1023-1032.

Drizin I, Gomtsyan A, Bayburt EK, Schmidt RG, Zheng GZ, Perner RJ, DiDomenico S, Koenig JR, Turner SC, Jinkerson TK, Brown BS, Keddy RG, McDonald HA, Honore P, Wismer CT, Marsh KC, Wetter JM, Polakowski JS, Segreti JA, Jarvis MF, et al. (2006) Structure-activity studies of a novel series of 5,6-fused heteroaromatic ureas as TRPV1 antagonists. Bioorg Med Chem 14:4740-4749.

Fride E, Mechoulam R (1993) Pharmacological activity of the cannabinoid 
receptor agonist, anandamide, a brain constituent. Eur J Pharmacol 231:313-314.

Garami A, Shimansky YP, Pakai E, Oliveira DL, Gavva NR, Romanovsky AA (2010) Contributions of different modes of TRPV1 activation to TRPV1 antagonist-induced hyperthermia. J Neurosci 30:1435-1440.

Gavva NR (2008) Body-temperature maintenance as the predominant function of the vanilloid receptor TRPV1. Trends Pharmacol Sci 29:550-557.

Gavva NR, Tamir R, Qu Y, Klionsky L, Zhang TJ, Immke D, Wang J, Zhu D, Vanderah TW, Porreca F, Doherty EM, Norman MH, Wild KD, Bannon AW, Louis JC, Treanor JJ (2005) AMG 9810 [(E)-3-(4-t-butylphenyl)$\mathrm{N}$-(2,3-dihydrobenzo[b][1,4] dioxin-6-yl)acrylamide], a novel vanilloid receptor 1 (TRPV1) antagonist with antihyperalgesic properties. J Pharmacol Exp Ther 313:474-484.

Gonsiorek W, Lunn C, Fan X, Narula S, Lundell D, Hipkin RW (2000) Endocannabinoid 2-arachidonyl glycerol is a full agonist through human type 2 cannabinoid receptor: antagonism by anandamide. Mol Pharmacol 57:1045-1050.

Gordon CJ (1994) 24-hour control of body temperature in rats. I. Integration of behavioral and autonomic effectors. Am J Physiol 267:R71-R77.

Hori T (1984) Capsaicin and central control of thermoregulation. Pharmacol Ther 26:389-416.

Huffman DM, Barzilai N (2009) Role of visceral adipose tissue in aging. Biochim Biophys Acta 1790:1117-1123.

Hunt JL, Zaretsky DV, Sarkar S, Dimicco JA (2010) Dorsomedial hypothalamus mediates autonomic, neuroendocrine, and locomotor responses evoked from the medial preoptic area. Am J Physiol Regul Integr Comp Physiol 298:R130-R140.

Iida T, Shimizu I, Nealen ML, Campbell A, Caterina M (2005) Attenuated fever response in mice lacking TRPV1. Neurosci Lett 378:28-33.

Inoue R, Jensen LJ, Shi J, Morita H, Nishida M, Honda A, Ito Y (2006) Transient receptor potential channels in cardiovascular function and disease. Circ Res 99:119-131.

Kanizsai P, Garami A, Solymár M, Szolcsányi J, Szelényi Z (2009) Energetics of fasting heterothermia in TRPV1-KO and wild type mice. Physiol Behav 96:149-154.

Kawabata F, Inoue N, Yazawa S, Kawada T, Inoue K, Fushiki T (2006) Effects of $\mathrm{CH}-19$ sweet, a non-pungent cultivar of red pepper, in decreasing the body weight and suppressing body fat accumulation by sympathetic nerve activation in humans. Biosci Biotechnol Biochem 70:2824-2835.

Lee J, Di Marzo V, Brotchie JM (2006) A role for vanilloid receptor 1 (TRPV1) and endocannabinnoid signalling in the regulation of spontaneous and L-DOPA induced locomotion in normal and reserpine-treated rats. Neuropharmacology 51:557-565.

Lesnikov VA, Tsvetkova IP (1985) Stereotaxic coordinates of the mouse hypothalamus (in Russian). Fiziol Zh SSSR Im I M Sechenova 71:798-804.

Lundbaek JA, Birn P, Tape SE, Toombes GE, Søgaard R, Koeppe RE 2nd, Gruner SM, Hansen AJ, Andersen OS (2005) Capsaicin regulates voltage-dependent sodium channels by altering lipid bilayer elasticity. Mol Pharmacol 68:680-689.

Mandadi S, Nakanishi ST, Takashima Y, Dhaka A, Patapoutian A, McKemy DD, Whelan PJ (2009) Locomotor networks are targets of modulation by sensory transient receptor potential vanilloid 1 and transient receptor potential melastatin 8 channels. Neuroscience 162:1377-1397.

Marsch R, Foeller E, Rammes G, Bunck M, Kössl M, Holsboer F, Zieglgänsberger W, Landgraf R, Lutz B, Wotjak CT (2007) Reduced anxiety, conditioned fear, and hippocampal long-term potentiation in transient receptor potential vanilloid type 1 receptor-deficient mice. J Neurosci 27:832-839.

McAllen RM, Tanaka M, Ootsuka Y, McKinley MJ (2010) Multiple thermoregulatory effectors with independent central controls. Eur J Appl Physiol 109:27-33.

Mills C, McMackin M, Jaffe R, Yu J, Zininberg E, Slee D, Gogas K, Bradbury M (2008) Effects of the transient receptor potential vanilloid 1 antagonist A-425619 on body temperature and thermoregulation in the rat. Neuroscience 156:165-174.

Morrison SF, Nakamura K, Madden CJ (2008) Central control of thermogenesis. Exp Physiol 93:773-797.

Motter AL, Ahern GP (2008) TRPV1-null mice are protected from dietinduced obesity. FEBS Lett 582:2257-2262.

Mount LE, Willmott JV (1967) The relation between spontaneous activity, metabolic rate and the 24 hour cycle in mice at different environmental temperatures. J Physiol 190:371-380.

Nikami H, Mahmoud ME, Shimizu Y, Shiina T, Hirayama H, Iwami M, Dosoky RM, Ahmed MM, Takewaki T (2008) Capsaicin pretreatment attenuates LPS-induced hypothermia through TRPV1-independent mechanisms in chicken. Life Sci 82:1191-1195.

Nilius B, Owsianik G, Voets T, Peters JA (2007) Transient receptor potential cation channels in disease. Physiol Rev 87:165-217.

Panlilio LV, Mazzola C, Medalie J, Hahn B, Justinova Z, Drago F, Cadet JL, Yasar S, Goldberg SR (2009) Anandamide-induced behavioral disruption through a vanilloid-dependent mechanism in rats. Psychopharmacology (Berl) 203:529-538.

Proulx K, Cota D, Castañeda TR, Tschöp MH, D’Alessio DA, Tso P, Woods SC, Seeley RJ (2005) Mechanisms of oleoylethanolamide-induced changes in feeding behavior and motor activity. Am J Physiol Regul Integr Comp Physiol 289:R729-R737.

Romanovsky AA (2007) Thermoregulation: some concepts have changed. Functional architecture of the thermoregulatory system. Am J Physiol Regul Integr Comp Physiol 292:R37-R46.

Romanovsky AA, Ivanov AI, Shimansky YP (2002) Selected contribution: ambient temperature for experiments in rats: a new method for determining the zone of thermal neutrality. J Appl Physiol 92:2667-2679.

Romanovsky AA, Almeida MC, Garami A, Steiner AA, Norman MH, Morrison SF, Nakamura K, Burmeister JJ, Nucci TB (2009) The transient receptor potential vanilloid- 1 channel in thermoregulation: a thermosensor it is not. Pharmacol Rev 61:228-261.

Rong W, Hillsley K, Davis JB, Hicks G, Winchester WJ, Grundy D (2004) Jejunal afferent nerve sensitivity in wild-type and TRPV1 knockout mice. J Physiol 560:867-881.

Rudaya AY, Steiner AA, Robbins JR, Dragic AS, Romanovsky AA (2005) Thermoregulatory responses to lipopolysaccharide in the mouse: dependence on the dose and ambient temperature. Am J Physiol Regul Integr Comp Physiol 289:R1244-R1252.

Sharif-Naeini R, Ciura S, Zhang Z, Bourque CW (2008) Contribution of TRPV channels to osmosensory transduction, thirst, and vasopressin release. Kidney Int 73:811-815.

Smart D, Gunthorpe MJ, Jerman JC, Nasir S, Gray J, Muir AI, Chambers JK, Randall AD, Davis JB (2000) The endogenous lipid anandamide is a full agonist at the human vanilloid receptor (hVR1). Br J Pharmacol 129:227-230.

Smith PB, Compton DR, Welch SP, Razdan RK, Mechoulam R, Martin BR (1994) The pharmacological activity of anandamide, a putative endogenous cannabinoid, in mice. J Pharmacol Exp Ther 270:219-227.

Snitker S, Fujishima Y, Shen H, Ott S, Pi-Sunyer X, Furuhata Y, Sato H, Takahashi M (2009) Effects of novel capsinoid treatment on fatness and energy metabolism in humans: possible pharmacogenetic implications. Am J Clin Nutr 89:45-50.

Steiner AA, Turek VF, Almeida MC, Burmeister JJ, Oliveira DL, Roberts JL, Bannon AW, Norman MH, Louis JC, Treanor JJ, Gavva NR, Romanovsky AA (2007) Nonthermal activation of transient receptor potential vanilloid-1 channels in abdominal viscera tonically inhibits autonomic cold-defense effectors. J Neurosci 27:7459-7468.

Suri A, Szallasi A (2008) The emerging role of TRPV1 in diabetes and obesity. Trends Pharmacol Sci 29:29-36.

Szallasi A, Nilsson S, Farkas-Szallasi T, Blumberg PM, Hökfelt T, Lundberg JM (1995) Vanilloid (capsaicin) receptors in the rat: distribution in the brain, regional differences in the spinal cord, axonal transport to the periphery, and depletion by systemic vanilloid treatment. Brain Res 703:175-183.

Szallasi A, Cortright DN, Blum CA, Eid SR (2007) The vanilloid receptor TRPV1: 10 years from channel cloning to antagonist proof-of-concept. Nat Rev Drug Discov 6:357-372.

Szelényi Z, Hummel Z, Szolcsányi J, Davis JB (2004) Daily body temperature rhythm and heat tolerance in TRPV1 knockout and capsaicin pretreated mice. Eur J Neurosci 19:1421-1424.

Szentirmai E, Kapás L, Sun Y, Smith RG, Krueger JM (2010) Restricted feeding-induced sleep, activity, and body temperature changes in normal and preproghrelin-deficient mice. Am J Physiol Regul Integr Comp Physiol 298:R467-R477.

Takeda Y, Ishida T, Tsutsui R, Toide K, Tanimoto-Mori S, Watanabe S, Kanai Y, Kamei C (2008) Studies on somnolence in the daytime caused by drugs used for neuropathic pain. J Pharmacol Sci 107:246-250. 
Tominaga M, Caterina MJ, Malmberg AB, Rosen TA, Gilbert H, Skinner K, Raumann BE, Basbaum AI, Julius D (1998) The cloned capsaicin receptor integrates multiple pain-producing stimuli. Neuron 21:531-543.

Tzavara ET, Li DL, Moutsimilli L, Bisogno T, Di Marzo V, Phebus LA, Nomikos GG, Giros B (2006) Endocannabinoids activate transient receptor potential vanilloid 1 receptors to reduce hyperdopaminergiarelated hyperactivity: therapeutic implications. Biol Psychiatry 59:508515.

Vogel Z, Barg J, Levy R, Saya D, Heldman E, Mechoulam R (1993) Anandamide, a brain endogenous compound, interacts specifically with cannabinoid receptors and inhibits adenylate cyclase. J Neurochem 61:352-355.

Watanabe K, Matsunaga T, Nakamura S, Kimura T, Ho IK, Yoshimura H, Yamamoto I (1999) Pharmacological effects in mice of anandamide and its related fatty acid ethanolamides, and enhancement of cataleptogenic effect of anandamide by phenylmethylsulfonyl fluoride. Biol Pharm Bull 22:366-370.

Weinert D, Waterhouse J (1998) Diurnally changing effects of locomotor activity on body temperature in laboratory mice. Physiol Behav 63:837-843.

Westerterp KR, Plasqui G (2009) Physically active lifestyle does not decrease the risk of fattening. PLoS One 4:e4745.

Wiley JL, Razdan RK, Martin BR (2006) Evaluation of the role of the arachidonic acid cascade in anandamide's in vivo effects in mice. Life Sci 80:24-35.

Wise LE, Shelton CC, Cravatt BF, Martin BR, Lichtman AH (2007) Assessment of anandamide's pharmacological effects in mice deficient of both fatty acid amide hydrolase and cannabinoid CB1 receptors. Eur J Pharmacol 557:44-48.

Zhang LL, Yan Liu D, Ma LQ, Luo ZD, Cao TB, Zhong J, Yan ZC, Wang LJ, Zhao ZG, Zhu SJ, Schrader M, Thilo F, Zhu ZM, Tepel M (2007) Activation of transient receptor potential vanilloid type-1 channel prevents adipogenesis and obesity. Circ Res 100:1063-1070.

Zygmunt PM, Petersson J, Andersson DA, Chuang H, Sørgård M, Di Marzo V, Julius D, Högestätt ED (1999) Vanilloid receptors on sensory nerves mediate the vasodilator action of anandamide. Nature 400:452-457. 